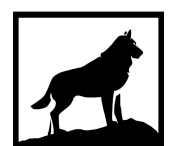

Michigan Technological

18 8 5 University
Michigan Technological University Digital Commons @ Michigan Tech

\title{
MODEL-BASED CONTROL OF HYBRID ELECTRIC POWERTRAINS INTEGRATED WITH LOW TEMPERATURE COMBUSTION ENGINES
}

Ali Soloukmofrad

Michigan Technological University, asoloukm@mtu.edu

Copyright 2017 Ali Soloukmofrad

\section{Recommended Citation}

Soloukmofrad, Ali, "MODEL-BASED CONTROL OF HYBRID ELECTRIC POWERTRAINS INTEGRATED WITH LOW TEMPERATURE COMBUSTION ENGINES", Open Access Dissertation, Michigan Technological University, 2017.

https://doi.org/10.37099/mtu.dc.etdr/384

Follow this and additional works at: https://digitalcommons.mtu.edu/etdr

Part of the Automotive Engineering Commons, Controls and Control Theory Commons, Energy Systems Commons, Heat Transfer, Combustion Commons, and the Power and Energy Commons 
MODEL-BASED CONTROL OF HYBRID ELECTRIC POWERTRAINS

INTEGRATED WITH LOW TEMPERATURE COMBUSTION ENGINES

By

Ali Soloukmofrad

\begin{abstract}
A DISSERTATION
Submitted in partial fulfillment of the requirements for the degree of DOCTOR OF PHILOSOPHY
\end{abstract}

In Mechanical Engineering-Engineering Mechanics

MICHIGAN TECHNOLOGICAL UNIVERSITY

2017

(C) 2017 Ali Soloukmofrad 

This dissertation has been approved in partial fulfillment of the requirements for the Degree of DOCTOR OF PHILOSOPHY in Mechanical Engineering-Engineering Mechanics.

Department of Mechanical Engineering-Engineering Mechanics

Dissertation Advisor: Dr. Mahdi Shahbakhti

Committee Member: Dr. John E Beard

Committee Member: Dr. Darrell L Robinette

Committee Member: Dr. Mohammad Shakiba-Herfeh

Department Chair: Dr. William W. Predebon 



\section{Dedication}

To my sister, Dr. Atefeh Solouk, for her contribution and dedication to biomaterial engineering,

my parents (Abbas and Mansoureh) for their love and support, my grandmother (Mama Narges) for her unconditional love, and those who have made my life a meaningful journey. 



\section{Contents}

List of Figures . . . . . . . . . . . . . . . . .

List of Tables . . . . . . . . . . . . . . . . . xxiii

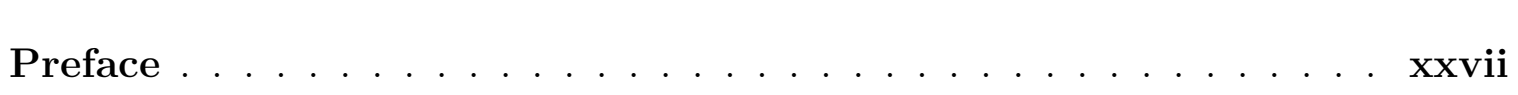

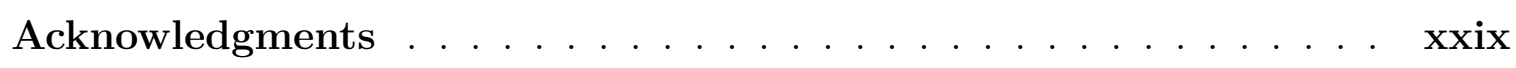

List of Abbreviations . . . . . . . . . . . . . . . xxxii

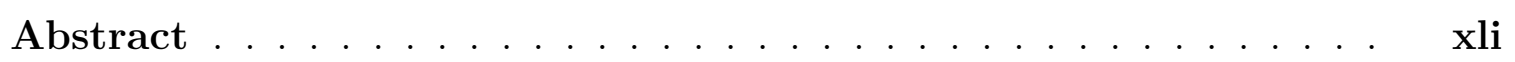

1 Chapter1: Introduction . . . . . . . . . . . . . . . 1

1.1 Shortcomings of State-of-the-art Research . . . . . . . . . . . 6

1.2 Objectives ......................................

2 Chapter2: Single-Mode HCCI Engine as Range Extender .... 11

2.1 Introduction . . . . . . . . . . . . . . . . . . . . . 12

$2.2 \quad$ HEV Modeling . . . . . . . . . . . . . . . . . . . 18

$2.2 .1 \quad$ HCCI Engine $\ldots \ldots \ldots \ldots$ 
2.2 .2 Battery . . . . . . . . . . . . . . . 20

2.2 .3 Traction E-motor _ . . . . . . . . . . . . . . . 23

2.2 .4 LVD Model . . . . . . . . . . . . . . . . . . . . . 24

2.2 .5 Vehicle Performance . . . . . . . . . . . . . . . 25

2.3 Energy Management Controller (EMC) Design . . . . . . . . . . 27

2.3.1 EMC Type I: Rule-Based Control (RBC) . . . . . . . . 2 27

2.3.2 EMC Type II: Global Optimization - Dynamic Programming

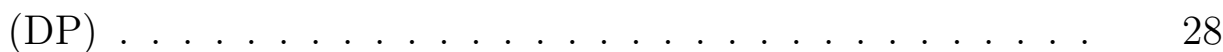

2.3.3 EMC Type III: Model Predictive Controller . . . . . . . . . 31

2.4 Results and Discussions . . . . . . . . . . . . . . . . 34

2.4.1 Validation of Model Components . . . . . . . . . . . 34

2.4.2 Single Mode Series HEV . . . . . . . . . . . . . . . . . . 35

2.4.3 Impact of Number of Engine Operating Points . . . . . . . 44

$2.4 .4 \quad$ E-REV . . . . . . . . . . . . . . . . . . . . 46

2.4.5 Impact of Driving Cycle . . . . . . . . . . . . . . . 49

2.5 Conclusions . . . . . . . . . . . . . . . . . . . . . 50

3 Chapter3: Single-Mode RCCI Engine as Range Extender . . . . 53

3.1 Introduction . . . . . . . . . . . . . . . . 54

$3.2 \quad$ SHEV Model Description . . . . . . . . . . . . . . . . . . . 60

3.2 .1 IC Engines . . . . . . . . . . . . . . . . . . . . 61

3.2.2 Longitudinal Vehicle Dynamics . . . . . . . . . . . . . 65 
3.2.3 E-motor Model . . . . . . . . . . . . . . . 66

3.2.4 Battery Model . . . . . . . . . . . . . . . . . 68

3.2.5 Vehicle Acceleration and Braking Performance . . . . . 69

3.2.6 Model Validation . . . . . . . . . . . . . . . 69

3.3 Selection of Engine Operating Points . . . . . . . . . . . . . 70

3.4 Energy Management Controller (EMC) Design . . . . . . . . . . 74

3.4 .1 EMC Type I: RBC . . . . . . . . . . . . . 74

3.4.2 EMC Type II: Global Optimization - DP . . . . . . . . . 75

3.4.3 EMC Type III: MPC . . . . . . . . . . . . . . . . 79

3.5 Results and Discussions . . . . . . . . . . . . . . 80

3.5.1 Prediction Time Horizon Sensitivity . . . . . . . . . . . . . 88

3.5.2 Initial SOC Sensitivity . . . . . . . . . . . . . . . 89

3.5.3 Driving Cycle Effect . . . . . . . . . . . . . . 93

3.6 Conclusions .................................. 94

4 Chapter4: Hybridized Multi-Mode LTC-SI Engine Experimental

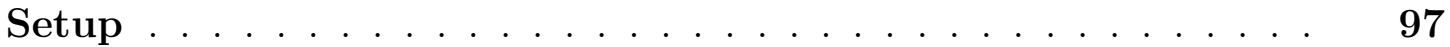

4.1 Electric Powertrain . . . . . . . . . . . . . . . . 99

4.2 E-Motor/Inverter . . . . . . . . . . . . . . . . . 102

4.2.1 E-motor Torque Tracking Performance . . . . . . . . . . 103

4.2.2 E-motor and Battery Characteristics Maps ........ 105

4.3 Multi-Mode Engine Setup . . . . . . . . . . . . . . . 107 
4.3.1 Engine Experimental BSFC and Temperature Maps . . . . . 110

4.3.2 Experimental Mode-Switching Fuel Penalty Map . . . . . . . 112

5 Chapter5: Multi-Mode LTC-SI Range Extender . . . . . . . . . . 119

5.1 Introduction . . . . . . . . . . . . . . . . . 120

5.2 Design of Optimal Control for Multi-Mode LTC-Based E-REV . . . 122

5.2.1 Pontryagin's Minimum Principal (PMP) .......... 124

5.3 Results .............................. 134

5.3.1 Single-Mode LTC Engine . . . . . . . . . . . . . 135

5.3.2 Multi-mode Engine . . . . . . . . . . . . . . 143

5.4 Conclusions .................................. 148

6 Chapter6: Multi-Mode LTC-SI in P2 Parallel HEV Architecture 151

6.1 Introduction . . . . . . . . . . . . . . . . 152

6.2 Design of Optimal Energy Management Control Strategy . . . . . . 155

6.2.1 Pontryagin's Minimum Principal (PMP) . . . . . . . . 156

6.2.1.1 Parallel P2 Architecture Model . . . . . . . . . 157

6.2.1.2 Development of PMP-based torque management strategy ................... . . . . 161

6.2.1.3 Extending the cost function for Multi-Mode operation ........................ 164

6.3 RESULTS ............................... 167

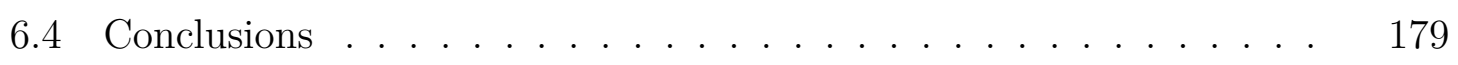


7 Chapter:7 Conclusion and Future Work

7.1 Summary and Conclusion . . . . . . . . . . . . . . 183

7.2 Suggestions for Future Work . . . . . . . . . . . . . . . . 190

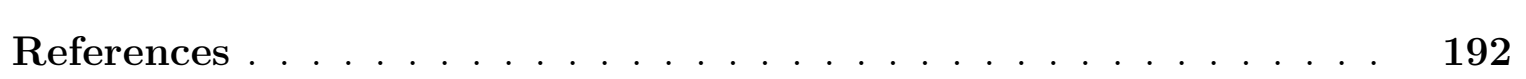

A Series HEV Components Sizing . . . . . . . . . . . . 207

A.1 Series HEV Components Sizing . . . . . . . . . . . . 207

A.1.1 Power Rating Design of Traction E-motor . . . . . . . . 207

A.1.2 Engine ........................ 210

A.1.3 Battery ........................... 211

A.1.3.1 Power Capacity ................ 2. 211

A.1.3.2 Energy Capacity ............. . . 212

B Electric Motor Experimental Setup . . . . . . . . . . . 215

B.1 Control Strategy Developed to Run the System . . . . . . . . . . 215

B.2 Battery ......................................

B.3 HV Box ........................... 220

B.4 Cooling System . . . . . . . . . . . . . . . 224

B.5 Battery Charger . . . . . . . . . . . . . . 226

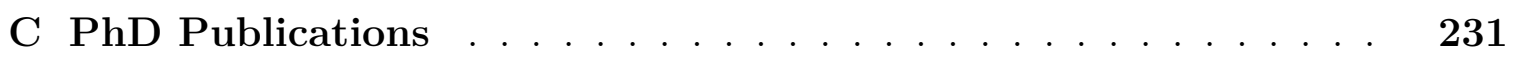

C.1 Peer Reviewed Journal Papers . . . . . . . . . . . . . . . 231

C.1.1 Published Journal Papers ............... 231 
C.1.2 Under-Review Journal Paper . . . . . . . . . . . . . . 232

C.1.3 Pre-Submission Journal Papers . . . . . . . . . . . . 232

C.2 Peer Reviewed Conference Papers . . . . . . . . . . . . . 233

C.3 Abstract Referred Conference Papers ............. . . 233

D Letters of Permission . . . . . . . . . . . . . . 237

D.1 Letter of Permission for 1$]$ (Chapter 2) . . . . . . . . . . 238

D.2 Letter of Permission for [2] (Chapter 3) . . . . . . . . . 240

D.3 Letter of Permission for Data Used in Figures 4.11 and 4.12 (Chapter 4) . . . . . . . . . . . . . . . . . . . . . . 241

D.4 Letter of Permission for [3, 4] (Chapters [5] and [6] . . . . . . 242

E Program and Data File Summary . . . . . . . . . . . . 243

E.1 Chapter 1 . . . . . . . . . . . . . . . . . . . . . . . 243

E.2 Chapter $2 \ldots \ldots \ldots \ldots \ldots \ldots \ldots$. . . . . . . . . . . . . . . . . 244

E.3 Chapter 3 . . . . . . . . . . . . . . . . . . . . . . . . 246

E.4 Chapter $4 \ldots \ldots \ldots \ldots \ldots \ldots$. . . . . . . . . . . . . . . . . 248

E.5 Chapter 5 . . . . . . . . . . . . . . . . . . . . . . 249

E.6 Chapter 6 . . . . . . . . . . . . . . . . . . . 250

E.7 Appendix $\mathrm{A} \ldots \ldots \ldots \ldots \ldots \ldots \ldots$. . . . . . . . . . . . . . . . . 252

E.8 Appendix B . . . . . . . . . . . . . . . . . . . . . . . 252 


\section{List of Figures}

1.1 Different types of ICEs and control techniques used in HEVs in previous studies. . . . . . . . . . . . . . . . . . .

1.2 Structure of this $\mathrm{PhD}$ dissertation . . . . . . . . . . . .

2.1 Different types of ICEs and EMCs used in HEVs in previous studies.

2.2 A schematic diagram to illustrate the power and control flow and main components of a series HEV . . . . . . . . . . . . . . .

2.3 HCCI engine power and BSFC variation as a function of engine speed and $\phi$.

2.4 Simulated acceleration and braking performance of the HEV in this

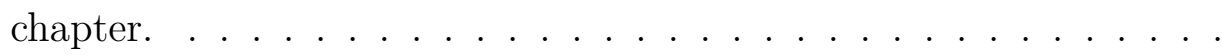

2.5 Comparison of E-motor speed and torque simulation results from forward and backward HEV models during Extra-Urban Driving Cycle (EUDC) driving cycle. . . . . . . . . . . . . . . . .

2.6 Battery model validation against experimental data from [5]. . . . .

2.7 Experimental validation of E-motor efficiency using experimental data from [6]. 
2.8 BSFC map of GM Ecotec 1.4L SI engine. The data from [7] was used to generate this Figure. . . . . . . . . . . . . . 37

2.9 Traction E-motor speed during the UDDS driving cycle. . . . . . . 38

2.10 E-motor operating points, shown with ' $+{ }^{\prime}$, plotted over E-motor efficiency contours. . . . . . . . . . . . . . . . . 39

2.11 Desired vehicle speed vs. actual vehicle speed. . . . . . . . . . 40

2.12 Engine on/off status and the battery SOC pattern in the DP and RBC strategies. ............................ 40

2.13 Power split profile in the DP controller. . . . . . . . . . . 41

2.14 Battery SOC variation in MPC for different Time Horizons (TH) and DP controllers. . . . . . . . . . . . . . . . . .

2.15 Fuel economy comparison for different EMCs including the effect of MPC Time Horizons (TH) in the series HEV for UDDS driving cycle.

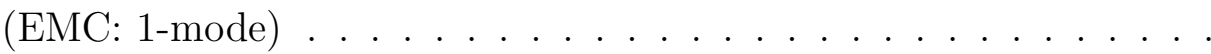

2.16 HEV fuel economy improvement by using HCCI versus SI as a function of number of engine operating points and fuel penalty for engine start-

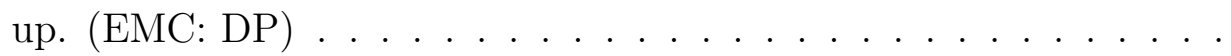

2.17 Engine on/off status in E-REV and series HEV. (EMC: 3-mode DP) 48

2.18 Fuel economy improvement by HCCI in different THs in series HEV and E-REV modes. (EMC: 3-mode) . . . . . . . . . . . . 
2.19 HEV fuel economy improvement by using HCCI versus SI in various driving cycles. (EMC: 3-mode DP) . . . . . . . . . .

3.1 Prior studies categorized based on different types of ICEs and EMCs incorporated in HEVs.

3.2 A schematic diagram to illustrate the power and control flows among the main components of an RCCI-based SHEV.

3.3 BSFC map $(\mathrm{g} / \mathrm{kWh})$ of the RCCI engine in this chapter. The data from [8] was used to generate this figure. . . . . . . . . . . .

3.4 BSFC map (g/kWh) of the GM Z19DTH CI (diesel) engine. The data from [8] was used to generate this figure. . . . . . . . . . .

3.5 BSFC map (g/kWh) of GM A14XFL SI (gasoline) engine. The data from [7] was used to generate this figure. . . . . . . . . . .

3.6 RCCI exhaust gas temperature map for selecting the RCCI engine operating points. The data from [8] was used to generate this figure.

3.7 The combined driving cycle used for the evaluation of the designed EMCs.

3.8 Fuel economy (FE) improvement by using RCCI versus a) SI and b) CI engines as a function of number of engine operating points and engine start-up fuel penalty. (EMC: DP) . . . . . . . . . .

3.9 Traction E-motor speed during the combined driving cycle. . . . . . 
3.10 E-motor operating points, shown with 'o' symbol, plotted over the Emotor efficiency map. . . . . . . . . . . . . .... 84

3.11 Desired vehicle speed vs. actual vehicle speed with root mean square tracking error of $0.8 \mathrm{mph} . \ldots \ldots \ldots \ldots$

3.12 Engine on/off status and the battery SOC pattern in the 3-mode DP

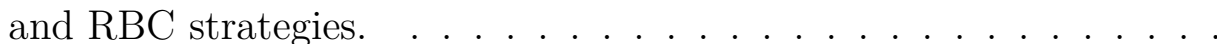

3.13 Power distribution in the SHEV during the combined driving cycle. (EMC: 3-mode DP) . . . . . . . . . . . . .

3.14 Battery SOC variations in MPC and DP controllers. (EMC: 3-mode)

3.15 Fuel economy (FE) comparison for a) RCCI, b) SI, and c) CI engines for different EMCs. . . . . . . . . . . . . . . . . . .

3.16 Comparison between battery energy loss for the low $S O C_{\text {initial }}$ and high $S O C_{\text {initial }}$ operating conditions. (EMC: 3-mode DP) . . . . .

3.17 The RCCI engine power pattern in the SHEV for two initial SOC operating conditions. (EMC: 3-mode DP) . . . . . . . . . .

3.18 Fuel economy (FE) improvement by using the RCCI engine over the SI or CI at two initial SOC operating conditions. (EMC: 3-mode MPC)

3.19 HEV fuel economy (FE) improvement by using the RCCI engine versus the SI and CI engines in four different driving cycles. (EMC: 3-mode

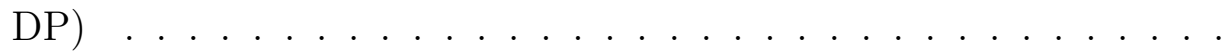


4.1 Developed LTC-based hybrid electric powertrain experimental testbed with a double-ended $465 \mathrm{hp}$ AC dynamometer at Michigan Technological University. . . . . . . . . . . . . . . . . . .

4.2 CAD model of the e-motor test setup in CATIA. . . . . . . . . .

4.3 Stress analysis in ANSYS for the e-motor mount design. . . . . . . 101

4.4 Electric powertrain components layout of the LTC-HEV testbed . . 102

4.5 E-motor traction test results. . . . . . . . . . . . 104

4.6 High voltage battery specifications: a) charge and discharge power limit, b) open circuit voltage (OCV), and c) internal resistance variation vs. SOC at battery temperature of $25^{\circ} \mathrm{C} \ldots \ldots . . . . .105$

4.7 Efficiency map of the $100 \mathrm{~kW}$ synchronous PMSM Remy motor including the transmission efficiency. Test condition: e-motor temperature $=45^{\circ} \mathrm{C}$ and DC bus voltage $=360 \mathrm{~V}$

4.8 Schematic of fuel-flexible multi-mode LTC-SI engine test setup. . . 108

4.9 Experimental BSFC map of the developed multi-mode LTC-SI engine. Data points are shown by dot symbols. . . . . . . . . . .

4.10 Experimental temperature map of the developed multi-mode LTC-SI engine. Data points are shown by dot symbols. . . . . . . . . . 
4.11 RCCI to SI mode transient experiment, where IMEP and MAP stand for indicated mean effective pressure and manifold absolute pressure, respectively. Engine speed $=1300 \mathrm{RPM}, T_{\text {intake }}=60{ }^{\circ} \mathrm{C}$ and remaining test conditions are the same as those in Table 4.5. The data in this figure was collected as part of the study in [9] by Jayant Arora. Permission letter is included in Appendix D. . . . . . . . . .

4.12 HCCI to SI mode transient experiment. Engine speed = 1200 RPM, $T_{\text {intake }}=60^{\circ} \mathrm{C}$ and remaining test conditions are the same as those in Table 4.5. The data in this figure was collected as part of the study in [9] by Jayant Arora. Permission letter is included in Appendix D. .

4.13 Experimental mode-switching fuel penalty map. . . . . . . . . . .

5.1 Different types of ICEs and control techniques used in HEVs in previous studies. . . . . . . . . . . . . . . . . . .

5.2 Series E-REV architecture in this chapter. PDU denotes power distri-

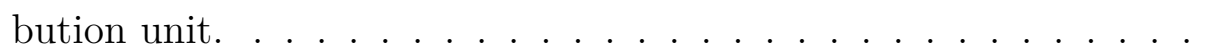

5.3 a) Finding the best engine speed at each power level based on the minimum BSFC (the green line shows the optimum engine speed); b) the engine lowest fuel consumption at each power level. . . . . . . .

5.4 Engine BSFC map and operating points for different ICE modes during the HWFET driving cycle. 
5.5 Optimum engine power $\left(P_{\text {eng,opt }}\right)$ of the a) HCCI, b) RCCI, and c) SI engines based on the vehicle speed and wheel power for the HWFET driving cycle. The white regions inside dashed lines show the conditions when the engine is $\mathrm{OFF} . \ldots \ldots \ldots \ldots$

5.6 Total work of the vehicle at wheels, the battery, and the single-mode engine for the UDDS (top row) and the HWFET driving cycles (bottom row). The x-axis is defined as: 1) $P_{\text {wheel }}>0$ and $\left.P_{b a t}>0,2\right) P_{\text {wheel }}>0$ and $\left.P_{b a t}<0,3\right) P_{w h e e l}<0$ and $\left.P_{b a t}>0,4\right) P_{w h e e l}<0$ and $P_{b a t}<0$.

5.7 Fuel economy of the series E-REV running with different single-mode engines (left) and fuel economy improvement over the SI engine (right). The results are for UDDS and HWFET driving cycles.

5.8 The engines' speed versus vehicle speed for the FT75 driving cycle. High noise area is shown by a dashed box in the subplots. . . . . . 144

5.9 Optimized multi-mode engine operation and effect of SI mode switching fuel penalty on the vehicle fuel economy and engine mode contributions. The results are shown when the fuel penalty is reduced by 50 , 90, and 95 percent compared to the baseline. . . . . . . . . . .

5.10 Optimized multi-mode engine operation and effect of $R C C I$ mode switching fuel penalty on the vehicle fuel economy and engine mode contributions. The results are shown when the fuel penalty is reduced by 50,90 , and 95 percent compared to the baseline. . . . . . . . . 147 
6.1 Different types of ICEs and control techniques used in HEVs in previous studies. . . . . . . . . . . . . . . . . .

6.2 Parallel HEV architecture in this chapter. . . . . . . . . . . 158

6.3 Engine operating points over the Single-modeengine BSFC map for different electrification levels for a) UDDS and b) HWFET driving cycles. . . . . . . . . . . . . . . . . . . . . 169

6.4 Engine operating points over the Multi-modeengine BSFC map for different electrification levels for a) UDDS and b) HWFET driving

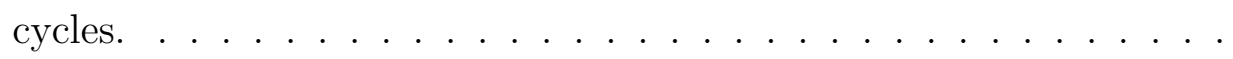

6.5 Mode distribution in two different driving cycles for three hybridization levels.

6.6 Fuel consumption for the multi-mode and single mode engines in two driving cycles and three electrification levels. . . . . . . . . . .

6.7 Running modes of the LTC-SI HEV in different driving cycles and electrification levels.

6.8 Engine operating points over the Single-modeengine exhaust gas temperature map for different electrification levels for a) UDDS and b) HWFET driving cycle. . . . . . . . . . . . . . 178

6.9 Engine operating points over the Multi-modeengine exhaust gas temperature map for different electrification levels for a) UDDS and b) HWFET driving cycle. 
A.1 Required power from the traction E-motor for the vehicle acceleration.

A.2 Required power from the traction E-motor for the vehicle gradability.

A.3 Different E-motors studied in this appendix. . . . . . . . . . .

A.4 Engine Required Power Rating at Constant Vehicle Speeds. . . . . .

B.1 Developed program for handling the setup startup/run and modelbased control of the e-motor setup.

B.2 Snapshot of the developed StateFlow handler programs for (a) battery (b) ) inverter. . . . . . . . . . . . . . . . .

B.3 Developed Battery Handler Software Layout . . . . . . . . . . . . .

B.4 HV box with its connected sub-systems. Details of the pre-charge circuit are also indicated in the figure. DIO in this figure refers to

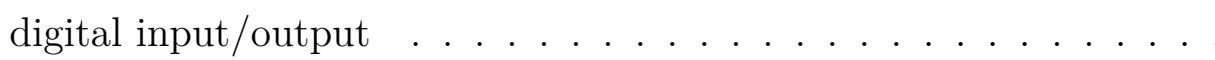

B.5 Electric circuit of the designed HV box the pre-charge circuit. . . .

B.6 E-motor cooling loop layout. . . . . . . . . . . . . .

B.7 Inverter cooling loop layout. . . . . . . . . . . . . . . .

B.8 Inverter cooling loop layout. . . . . . . . . . . . . . .

B.9 BRUSA NLG514 $3.3 \mathrm{~kW}$ battery charger. . . . . . . . . . . . . .

B.10 RS232 wire used for communicating with the charger cub-controller.

B.11 Battery charging profile.

B.12 Hyper Terminal software interface is used to send and read the charger controller signals. 
C.1 SAE best presentation award received at SAE 2016 International Powertrains, Fuels, and Lubricants for reference [10]. . . . . . . . . . 234

C.2 Paper [10] from this thesis is highlighted in GreenCarCongress.com, well-recognized media in the area of automotive engineering [10]. [URL: http://www.greencarcongress.com/2016/11/2016.html] . . .

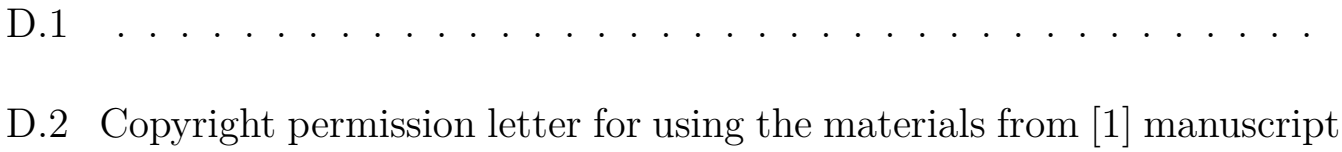
used in Chapter $2 \ldots \ldots \ldots \ldots \ldots \ldots$

D.3 Copyright permission letter for using the materials from [2] manuscript used in Chapter 3 . . . . . . . . . . . . . . . . . . . . 240

D.4 Permission letter from Jayant Arora for using the data in Figures 4.11 and4.12 as part of the study in [9]. (Chapter 4) . . . . . . . . 241

D.5 Copyright permission for using the materials from [3, 4] manuscript used in Chapters 5 and 6 . . . . . . . . . . . . . . . 242 


\section{List of Tables}

2.1 HCCI engine parameters. . . . . . . . . . . .

2.2 LV30P SAFT Li-ion battery parameters. $\ldots \ldots \ldots \ldots \ldots$

2.3 Parameters for UQM PowerPhase 75 traction E-motor model. . . .

2.4 Vehicle specifications.

2.5 Fuel economy comparison for different EMCs including the effect of MPC Time Horizons (TH) in series HEV. (EMC: 3-mode) . . . . .

2.6 Fuel economy comparison for different EMCs including the effect of MPC Time Horizons (TH) in E-REV mode. (EMC: 3-mode) . . . .

2.7 Average power without full regenerative braking in three driving cy-

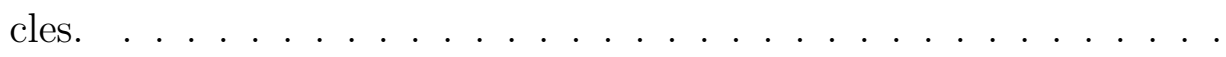

3.1 Vehicle specifications. . . . . . . . . . . . . . .

3.2 Specification of GM Z19DTH diesel engine converted for RCCI operation [8]

3.3 Engine-out $N O_{x}$ emissions in RCCI and CI engines operating points. The data is from [8]. 
3.4 RCCI engine fuel economy values in the SHEV architecture as a function of engine start-up fuel penalty and number of operating modes

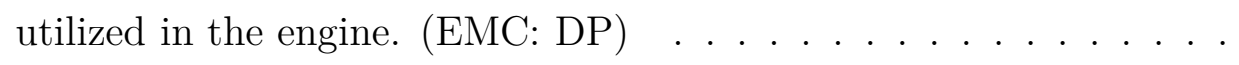

3.5 Fuel economy comparison for different EMCs including the effect of MPC Time Horizon (TH) in the $\underline{\mathrm{SHEV} \text { with } S O C_{\text {initial }}=0.3}$. (EMC:

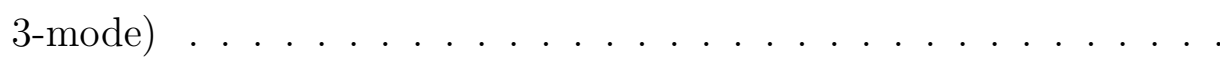

3.6 Average power in the studied driving cycles. . . . . . . . . . . .

4.1 Battery specifications

4.2 E-motor specifications. . . . . . . . . . . . . . . 103

4.3 Inverter specifications. . . . . . . . . . . . . . . . . 103

4.4 Parameters of the baseline engine in this study . . . . . . . . 107

4.5 Engine test setting for SI and LTC operating modes . . . . . . . . 110

5.1 Vehicle specifications. . . . . . . . . . . . . 125

5.2 Series E-REV performance results for using a single-mode engine during the UDDSdriving cycle . . . . . . . . . . . . . . . 136

5.3 Series E-REV performance results for using a single-mode engine during the HWFETdriving cycle . . . . . . . . . . . . . . . . . 138

6.1 Vehicle specifications. . . . . . . . . . . . . . . 158

6.2 Definition of hybridization levels in this chapter ........ 167 
6.3 Results for both multi-mode LTC-SI and single-mode SI engine in different electrification levels during UDDSdriving cycle . . . . . . . . 169

6.4 Results for both multi-mode LTC-SI and single-mode SI engine in different electrification levels during HWFETdriving cycle . . . . . . 170

A.1 Parameters of the battery (i.e., LV30P SAFT) selected in this appendix.

B.1 HV Box bill of materials and specifications. . . . . . . . . . .

B.2 E-motor cooling system specifications.

B.3 Battery charger specifications.

E.1 Chapter 1 figure files. . . . . . . . . . . . . . . . . 243

E.2 Chapter 2 figure files. . . . . . . . . . . . . . . . 244

E.3 Required data files. . . . . . . . . . . . . . . . 245

E.4 MATLAB script and SIMULINK files. . . . . . . . . . . . 245

E.5 Chapter 3 figure files. . . . . . . . . . . . . . . . . . . . . 246

E.6 MATLAB script and SIMULINK files. . . . . . . . . . . 247

E.7 Chapter 4 figure files. . . . . . . . . . . . . . . . 248

E.8 Experimental data files for tests. . . . . . . . . . . . . 248

E.9 Chapter 5 figure files. . . . . . . . . . . . . . . . . . . 249

E.10 Required data files. . . . . . . . . . . . . . . . 249

E.11 MATLAB script and Simulink files. . . . . . . . . . . . 250 
E.12 Chapter 6 figure files. . . . . . . . . . . . . . . . . 250

E.13 Required data files. . . . . . . . . . . . . . . . . . 251 25

E.14 MATLAB script and Simulink files. . . . . . . . . . . . 251

E.15 Appendix $₫$ figure files. . . . . . . . . . . . . . . 252

E.16 Appendix $\mathrm{A}$ figure files. . . . . . . . . . . . . . . . . . . 252 


\section{Preface}

The manuscript and the results of this dissertation are mainly based on five journal papers and five refereed conference papers (see appendix $\mathrm{C}$ for details). The contribution of the author of this dissertation as well as the contributions of the co-authors of the papers are as follows:

- Contributions for Chapters 2 and $33([1,[2])$ : Designing the optimization framework, modeling, simulations, analysis of the results, and manuscript writing are done by the author of this dissertation, A. Solouk. Dr. M. Shahbakhti provided guidance and contributed in discussions for analysis of the results and the manuscript editing.

- Contributions for Chapters 5 and $6[([10])$ : E-motor data collection, designing the optimization framework, modeling, simulations, analysis of the results, and manuscript writing were done by the author of this dissertation. Dr. M. Shakiba provided guidance for developing the optimization algorithm and contributed in analysis of the results. Dr. M. Shahbakhti contributed in discussions, analysis of the results, and editing the manuscript.

Moreover, the contribution of the collaborative works are as follows: 
- Collaborative work contribution for Chapter 4 and appendix B: E-motor experimental setup was designed and developed by major contributions of different graduate students and the author of this dissertation was leading the team and was involved in every details of the setup development and fabrication. The HV box was designed by A. Rezaei, who also provided guidance for developing the software for running the e-motor. S. Viswanathan and N. Ghike were involved in diagnosing the battery CAN communications. Moreover, S. Viswanathan and S. Mohan assisted in programming and operating the battery charger. M. Cheruvathur and D. Xiong were invloved in the CAD design of the E-motor setup and the e-motor mount stress analysis was conducted by P. Meruva. In addition, on the LTC engine experimental side, K. Kannan, Dr. S. Polat, J. Arora, and Dr. H. Solmaz were the major contributors for the engine experimental setup development and data collection. Dr. M. Shahbakhti and P. Dice contributed in the experimental setup development by providing feedback on the setup progress through holding weekly meetings. 


\section{Acknowledgments}

First and foremost I would like to thank my advisor Dr. Mahdi Shahbakhti for providing me with this opportunity to join his research group. He prepared an atmosphere for me to grow and develop my professional skills including, research, leadership, and mentorship. I found my strength and weakness points which helped me to find my future professional path. Moreover, his enthusiasm and courage motivated me to deliver results in the form of journal and conference articles. I appreciate all his contributions of time, fund, and ideas to make my $\mathrm{PhD}$ experience productive and stimulating.

I would like to thank Dr. Friedrich, director of graduate studies of ME-EM department, and the ME-EM department for financially supporting my $\mathrm{PhD}$ through Teaching Assistantships and Tuition Fellowships.

Special thanks to my mentor and PhD committee member, Dr. Mohammad Shakiba, for his valuable contributions and feedbacks on the research of this dissertation. Dr. John Beard, Dr. Darrell Robinette, and Dr. Gordon Parker have been valuable examination committee members to develop this $\mathrm{PhD}$ research.

I graciously acknowledge Dr. A. Rezaei, S. Viswanathan, S. Mohan, and M. Cheruvathur for their great contribution in developing the experimental test setup. Their 
efforts significantly contributed to the quality of this dissertation.

My time at MTU was made enjoyable in large part due to the many friends. I am grateful for time spent with my friends, for my backpacking buddies and our memorable trips into the woods, for Ehsan Ansari and his lovely wife Maryam Khaksari, Soroush Sepahyar, Jeremy Dobbs, Troy Bouman and his lovely wife Andrea Bouman, Amir Rezaei, Meysam Razmara, Amir Khameneian and his lovely wife Sara Sadeghzadeh, Behdad Afkhami, Mehdi Mortazavi, and Ehsan Taheri. I have had memorable moments with my roommates, Farzad Ferdowsi and MReza Amini, which helped me to learn about myself.

Thanks to my friends: Ahmad Cheshmberah, Amir Rahmanpour, Mehdi Yeganeh, Rahim Solouk, Reza Solouk, and Ali Shafiekhani for being a "friend in need". My high school mathematics teacher, Abbas Jahazian, who motivated me to become interested into the mathematics world which ended up me being an engineer.

I can not thank enough to my Mahsa who has always been a source of love and passion. Her smile and energy has changed my life forever.

Lastly, many thanks to my parents who helped me to pursue my dreams and trusted in me to be an independent decision maker and find my life path; my only sister who was my first motivation to pursue this journey; my niece, Delaram, who has changed my vision for love, even though, I have not had a chance to hug her yet; my 
brother-in-law for being such a supportive person for my family. Mama Narges for her unconditional love. My grandfather, Reza, for his lifelong sacrifices to promote his family; I grow up with his valuable advices which helped me to become familiar with real life values at the very early stages of my life. 


\section{List of Abbreviations}

\begin{tabular}{|c|c|}
\hline Acronyms & Description \\
\hline AER & All electric range \\
\hline $\mathrm{BSFC}$ & Brake specific fuel consumption \\
\hline BTE & Brake thermal efficiency \\
\hline CI & Compression ignition \\
\hline $\mathrm{DP}$ & Dynamic programing \\
\hline DOC & Diesel Oxidation Catalysts \\
\hline E-REV & Extended range electric vehicle \\
\hline EMC & Energy management control \\
\hline EUDC & Extra-urban driving cycle \\
\hline ECMS & Equivalent consumption minimization strategy \\
\hline EV & Electric vehicle \\
\hline $\mathrm{EVC}$ & Exhaust valve closing \\
\hline $\mathrm{FE}$ & Fuel economy \\
\hline $\mathrm{HEV}$ & Hybrid electric vehicle \\
\hline HCCI & Homogeneous charge compression ignition \\
\hline HWFET & Highway fuel economy cycle \\
\hline ICE & Internal combustion engine \\
\hline IVO & Intake valve opening \\
\hline
\end{tabular}




\begin{tabular}{|c|c|}
\hline LTC & Low temperature combustion \\
\hline $\mathrm{LDV}$ & Low duty vehicle \\
\hline LVD & Longitudinal vehicle dynamics \\
\hline $\mathrm{MPC}$ & Model predictive control \\
\hline MPG & Miles per gallon \\
\hline MPRR & Maximum pressure rise rate \\
\hline $\mathrm{NOx}$ & Nitrogen oxides \\
\hline NEDC & New European driving cycle \\
\hline $\mathrm{OCV}$ & Open circuit voltage \\
\hline PHEV & Plug-in hybrid electric vehicle \\
\hline PCCI & Premixed charge compression ignition \\
\hline $\mathrm{PM}$ & Particulate matter \\
\hline PMP & Pontryagin minimum principle \\
\hline $\mathrm{PR}$ & Premixed ratio \\
\hline RCCI & Reactivity controlled compression ignition \\
\hline RMSE & Root mean square error \\
\hline $\mathrm{RBC}$ & Rule-based controller \\
\hline $\mathrm{RON}$ & Research octane number \\
\hline $\mathrm{SDC}$ & Stochastic dynamic programming \\
\hline $\mathrm{SOC}$ & State of charge \\
\hline $\mathrm{SOI}$ & Start of injection \\
\hline SI & Spark ignition \\
\hline
\end{tabular}


(1)

SUV Sport utility vahicle

TH Time horizon

UDDS Urban dynamometer driving schedule

VMT Vehicle miles travel 


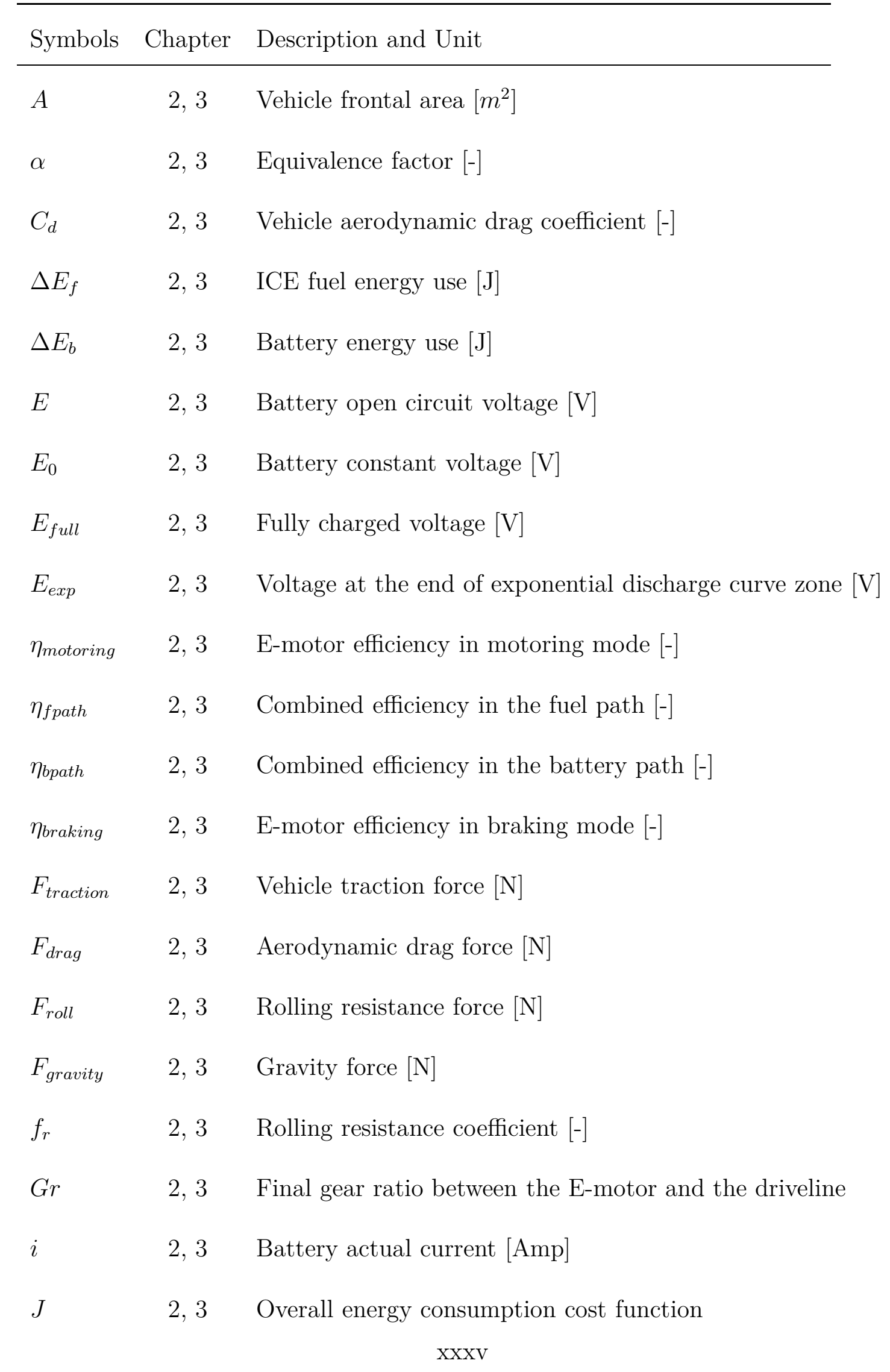




\begin{tabular}{|c|c|c|}
\hline$K$ & 2,3 & Polarization voltage $[\mathrm{V}]$ \\
\hline$M$ & 2, 3 & Vehicle total mass $[\mathrm{kg}]$ \\
\hline$\omega$ & [2, 3 & Engine speed $[\mathrm{rpm}]$ \\
\hline$\omega_{\text {motor }}$ & [2, 3 & E-motor speed $[\mathrm{rpm}]$ \\
\hline$P_{d}$ & [2, 3 & Driver power demand $[k W]$ \\
\hline$P_{\text {eng }}$ & 2,3 & Engine generated power $[k W]$ \\
\hline$P_{d, e n g}$ & [2, 3 & Engine demand power $[k W]$ \\
\hline$P_{p p s}$ & 2, 3 & Generated peak power source $[k W]$ \\
\hline$P_{d, p p s}$ & 2, 3 & Demanded peak power source $[k W]$ \\
\hline$P_{\text {gen }(1)}$ & [2, 3 & Generated power from the generator to supply the E-motor $[k W]$ \\
\hline$P_{g e n(2)}$ & 2,3 & Generated power from the generator to charge the battery $[k W]$ \\
\hline$P_{\text {motor }}$ & [2, 3 & Motor generated power $[k W]$ \\
\hline$P_{\text {available }}$ & 2, 3 & Available electrical power in the battery $[\mathrm{kW}]$ \\
\hline$P_{\text {grade }}$ & [2, 3 & Required traction power for hill climbing $[\mathrm{kW}]$ \\
\hline$P_{t}$ & 2, 3 & Required traction power for acceleration $[\mathrm{kW}]$ \\
\hline$P_{b}$ & [2, 3 & Maximum rated power of the battery $[\mathrm{kW}]$ \\
\hline$P_{g e n}$ & [2, 3 & Power of the engine/generator system $[\mathrm{kW}]$ \\
\hline$P_{r e q}$ & 2, 3 & Driver requested power $[\mathrm{kW}]$ \\
\hline$P_{\text {fpath }}$ & [2, 3 & Required engine power $[\mathrm{kW}]$ \\
\hline$P_{\text {bpath }}$ & 2, 3 & Required battery power $[\mathrm{kW}]$ \\
\hline$\phi$ & 2,3 & Fuel-air equivalence ratio [-] \\
\hline
\end{tabular}




\begin{tabular}{|c|c|c|}
\hline$Q$ & 2, 3 & Battery nominal capacity [Ah] \\
\hline$R$ & 2,3 & Battery internal resistance $[\mathrm{ohm}]$ \\
\hline$R$ & 2,3 & Wheel radius $[\mathrm{m}]$ \\
\hline$\rho$ & 2,3 & Air density $\left[\frac{\mathrm{kg}}{\mathrm{m}^{3}}\right]$ \\
\hline$S$ & 2,3 & Minimum energy consumption $[\mathrm{J}]$ \\
\hline$T_{\text {eng }}$ & 2,3 & Engine generated torque $[\mathrm{Nm}]$ \\
\hline$T_{\text {motor }}$ & 2,3 & E-motor applied torque to the wheels $[\mathrm{Nm}]$ \\
\hline$\theta$ & 2,3 & Road slope [degree] \\
\hline$t_{a}$ & 2,3 & Acceleration time $[\mathrm{sec}]$ \\
\hline$u$ & 2,3 & Control variable \\
\hline$V_{\text {vehicle }}$ & 2,3 & Actual vehicle speed $[\mathrm{mph}]$ \\
\hline$V_{b}$ & 2,3 & Vehicle speed corresponding to the E-motor base speed $\left[\frac{\mathrm{m}}{\mathrm{sec}}\right]$ \\
\hline$V_{f}$ & 2,3 & Final vehicle speed after acceleration $\left[\frac{\mathrm{m}}{\mathrm{sec}}\right]$ \\
\hline$x$ & 2 & E-motor speed ratio [-] \\
\hline$P_{d}$ & 5. 6 & Driver power demand $[k W]$ \\
\hline$P_{\text {eng }}$ & 5. 6 & Engine generated power $[k W]$ \\
\hline$P_{d, e n g}$ & 5,6 & Engine demand power $[k W]$ \\
\hline$P_{p p s}$ & 5. 6 & Generated peak power source $[k W]$ \\
\hline$P_{d, p p s}$ & 5. 6 & Demanded peak power source $[k W]$ \\
\hline$P_{g e n(1)}$ & 5. 6 & Generated power from the generator to supply the E-motor $[k W]$ \\
\hline
\end{tabular}




\begin{tabular}{|c|c|c|}
\hline$P_{g e n(2)}$ & 5. 6 & Generated power from the generator to charge the battery $[k W]$ \\
\hline$P_{\text {motor }}$ & 5. 6 & Motor generated power $[k W]$ \\
\hline$P_{\text {available }}$ & [5, 6 & Available electrical power in the battery $[\mathrm{kW}]$ \\
\hline$P_{r e q}$ & 5. 6 & Driver requested power $[\mathrm{kW}]$ \\
\hline$P_{\text {fpath }}$ & 5. 6 & Required engine power $[\mathrm{kW}]$ \\
\hline$P_{b p a t h}$ & 5. 6 & Required battery power $[\mathrm{kW}]$ \\
\hline$Q$ & 5, 6 & Battery nominal capacity $[\mathrm{Ah}]$ \\
\hline$R$ & [5, 6 & Battery internal resistance $[\Omega]$ \\
\hline$R_{w}$ & 5, 6 & Wheel radius $[\mathrm{m}]$ \\
\hline$\rho$ & 5. 6 & Air density $\left[\frac{\mathrm{kg}}{\mathrm{m}^{3}}\right]$ \\
\hline$S$ & [5, 6] & Minimum energy consumption $[\mathrm{J}]$ \\
\hline$T_{\text {eng }}$ & 5. 6 & Engine generated torque $[\mathrm{Nm}]$ \\
\hline$T_{\text {motor }}$ & 5. 6 & E-motor applied torque to wheels $[\mathrm{Nm}]$ \\
\hline$V$ & [5, 6 & Actual vehicle speed [mph] \\
\hline$\lambda$ & 5, 6 & Co-state [-] \\
\hline$C_{d}$ & 5, 6 & Vehicle aerodynamic drag coefficient [-] \\
\hline$\dot{m}_{f}$ & 5, 6 & Fuel consumption rate $[\mathrm{g} / \mathrm{sec}]$ \\
\hline$f_{r}$ & 5,6 & Rolling resistance coefficient [-] \\
\hline$\omega$ & [5, 6 & Engine speed $[\mathrm{rpm}]$ \\
\hline$\omega_{\text {motor }}$ & 5. 6 & E-motor speed $[\mathrm{rpm}]$ \\
\hline$A$ & 5. 6 & Vehicle frontal area $\left[m^{2}\right]$ \\
\hline
\end{tabular}




\begin{tabular}{|c|c|c|}
\hline $\mathcal{H}$ & 5. 6 & Hamiltonian $[g / s e c]$ \\
\hline$M$ & 5,6 & Vehicle total mass $[k g]$ \\
\hline$n_{c}$ & 5,6 & Mechanical coupling ration [-] \\
\hline$n_{t}$ & 5, 6 & Transmission ratio [-] \\
\hline$n_{d}$ & 5,6 & Differential ratio [-] \\
\hline$P_{\text {motor,mech }}$ & 5. 6 & Traction mechanical power of e-motor $[k W]$ \\
\hline$P_{\text {motor }, e}$ & 5 , 6 & Regen electrical power of e-motor $[k W]$ \\
\hline$P_{b a t}$ & 5 , 6 & Battery power $[k W]$ \\
\hline$P_{\text {wheel }}$ & 5,6 & Power demand at wheel $[k W]$ \\
\hline$P_{\text {eng }}$ & 5. 6 & Engine generated power $[k W]$ \\
\hline$Q_{\text {nom }}$ & 5. 6 & Battery nominal energy capacity $[W h]$ \\
\hline$R$ & [5, 6 & Battery internal resistance $[\Omega]$ \\
\hline$V_{v e h}$ & 5,6 & Vehicle speed $\left[\frac{m}{s e c}\right]$ \\
\hline$r$ & 5,6 & Wheel radius $[m]$ \\
\hline$\rho$ & 5, 6 & Air density $\left[\frac{\mathrm{kg}}{\mathrm{m}^{3}}\right]$ \\
\hline$\theta$ & 5, 6 & Road slope $\left[^{\circ}\right]$ \\
\hline$m_{i j}$ & 5, 6 & Mode-switching fuel penalty $[g]$ \\
\hline$u$ & 5,6 & Control variable \\
\hline
\end{tabular}





\section{Abstract}

Powertrain electrification including hybridizing advanced combustion engines is a viable cost-effective solution to improve fuel economy of vehicles. This will provide opportunity for narrow-range high-efficiency combustion regimes to be able to operate and consequently improve vehicle's fuel conversion efficiency, compared to conventional hybrid electric vehicles (HEV)s. Low temperature combustion (LTC) engines offer the highest peak brake thermal efficiency reported in literature, but these engines have narrow operating range. In addition, LTC engines have ultra-low soot and nitrogen oxides (NOx) emissions, compared to conventional compression ignition and spark ignition (SI) engines. This dissertation concentrates on integrating the LTC engines (i) in series HEV and extended range electric vehicle (E-REV) architectures which decouple the engine from the drivetrain and allow the ICE to operate fully in a dedicated LTC mode, and (ii) a parallel HEV architecture to investigate optimum performance for fuel saving by utilizing electric torque assist level offered by e-motor. An electrified LTC-SI powertrain test setup is built at Michigan Technological University to develop the powertrain efficiency maps to be used in energy management control (EMC) framework.

Three different types of Energy Management Control (EMC) strategies are developed. The EMC strategies encompass thermostatic rule-based control (RBC), offline (i.e., 
dynamic programing (DP) and pontryagin's minimum principal (PMP)), and online optimization (i.e., model predictive control (MPC)). The developed EMC strategies are then implemented on experimentally validated HEV powertrain model to investigate the powertrain fuel economy. A dedicated single-mode homogeneous charge compression ignition (HCCI) and reactivity controlled compression ignition (RCCI) engines are integrated with series HEV powertrain. The results show up to $17.7 \%$ and $14.2 \%$ fuel economy saving of using HCCI and RCCI, respectively in series HEV compared to modern SI engine in the similar architecture. In addition, the MPC results show that sub-optimal fuel economy is achieved by predicting the vehicle speed profile for a time horizon of $70 \mathrm{sec}$.

Furthermore, a multi-mode LTC-SI engine is integrated in both series and parallel HEVs. The developed multi-mode LTC-SI engine enables flexibility in combustion mode-switching over the driving cycle, which helps to improve the overall fuel economy. The engine operation modes include HCCI, RCCI, and SI modes. The powertrain controller is designed to enable switching among different modes, with minimum fuel penalty for transient engine operations. In the parallel $\mathrm{HEV}$ architecture, the results for the UDDS driving cycle show the maximum benefit of the multi-mode LTCSI engine is realized in the mild electrification level, where the LTC mode operating time increases dramatically from $5.0 \%$ in Plug-in Hybrid Electric Vehicle (PHEV) to $20.5 \%$ in mild HEV. 


\section{Chapter 1}

\section{Introduction}

The U.S. light-duty (LD) regulations require a fleet average of 4.3 liter/100 km by 2025 in order to meet the $101 \mathrm{~g} / \mathrm{km} \mathrm{CO}_{2}$ level [11]. In addition, in the European Union, the average fleet fuel consumption regulations for the new cars require 4.1 liter/100 km by 2021 [12]. High efficiency engines along with powertrain electrification will play a critical role in meeting such stringent goals from the cost-effectiveness perspective [13, 14, 15]. Currently, the spark-ignition (SI) engine fueled with gasoline is the primary engine used in the LD vehicles in the U.S. [11]. Conventional compression ignition (CI) engines are noteworthy for the LD vehicles due to their higher efficiency. However, the CI engines require an expensive and complex aftertreatment system for particular matter (PM) and $N O_{x}$ control [16]. To improve vehicular fuel 
economy and reduce aftertreatment expenses, various studies have investigated advanced combustion regimes to achieve higher thermal efficiencies than those in CI engines while diminishing engine-out emissions [17, 18, 19]. A promising advanced combustion regime is low temperature combustion (LTC), and consists of a family of variants including homogeneous charge compression ignition (HCCI), reactivity controlled compression ignition (RCCI), and partially-premixed charge compression ignition (PCCI) [20, 21]. LTC engines can offer peak indicated thermal efficiency of $53 \%$ [19] with ultra low $N O_{x}$ and PM engine-out emissions [19].

Even though the LTC engines benefit from higher thermal efficiencies and relatively inexpensive aftretreatment systems, they have narrow operating ranges and often require more complex combustion control which makes them challenging and less desirable for automotive powertrains. In addition, as the fleet merges to a higher degree of powertrain electrification path, more opportunities for advanced combustion regimes (i.e., LTC) will arise. It is because the powertrain electrification allows the engine to be downsized and operated in a narrow-range high-efficiency combustion regimes as compared to the conventional powertrains. In order to tackle these two challenges, this $\mathrm{PhD}$ dissertation investigates integration of the LTC engines with hybrid electric powertrains in order to minimize the LTC engine transitions and improve the operating range by taking advantage of powertrain hybridization. To do this, powetrain hybridization is investigated for i) Series HEV (SHEV), and ii) parallel HEV with different hybridization levels. In the SHEV architecture, the engine is decoupled from 
the drivetrain, which allows the ICE to operate in a narrow operating range. SHEV architectures also remove the engine mode transients, therefore, these architectures tackle the two major challenges of the LTC engine by adding electric components cost to the powertrain. There are currently some SHEVs and E-REVs on the market such as the Chevrolet Volt, Fisker Karma, BMWi3. In addition, integrating the LTC engines with parallel HEV architecture, decreases the ICE mode transitions by using e-motor torque assist. Thus, the parallel HEV architecture improves the LTC narrow operating range and facilitates implementation of this promising fuel-efficient powertrain, while adding lower cost to the powertrain. Chevrolet Malibu, Chevrolet Impala, Buick Regal, Mercedes-Benz S400 are some examples of the parallel HEV architectures.

Figure 1.1 categorizes the prior hybrid electric vehicle (HEV) studies based on different engine types including conventional (i.e., SI, CI, Atkinson) and advanced combustion engines (i.e., LTC). In the first category, conventional SI and CI engines have been used in different HEV architectures. The SI engines have been integrated in HEV and range extender architectures [22, 23, 24, 25, 26]. Atkinson SI engines are popular in the market and are used in the Toyota Prius, Ford C-Max, Lexus RX 450h, and Honda Accord. In reference [27, 28], Toyota has achieved 10\% lower fuel consumption compared to SI engine by converting the Honda Accords PHEV SI engine to the Atkinson cycle. The CI engine mostly have been integrated in trucks and Sport Utility Vehicles (SUVs) [29, 30, 31, 32, 33]. 


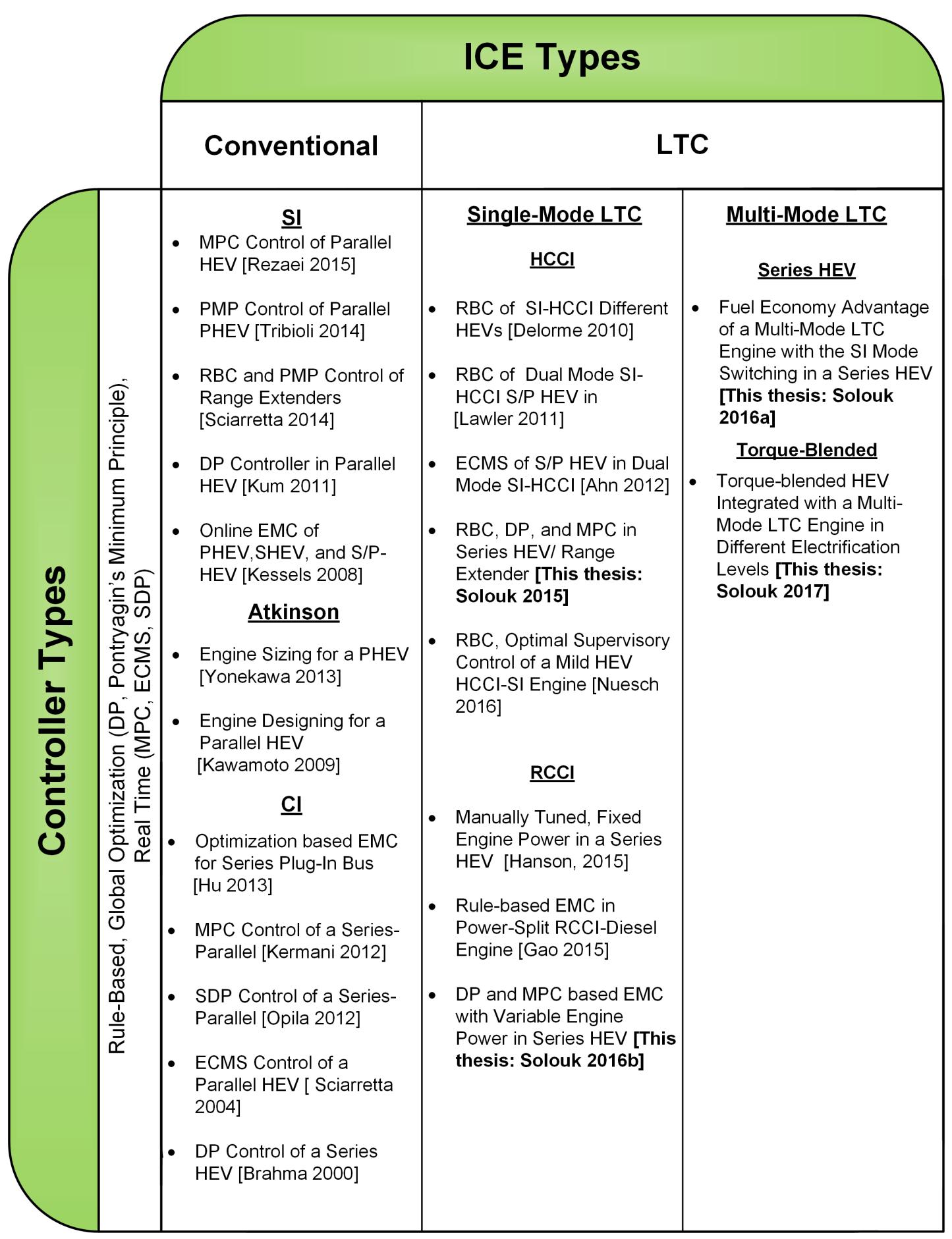

Figure 1.1: Different types of ICEs and control techniques used in HEVs in previous studies. 
In the second category, the LTC engines are integrated in different HEV architectures. Few studies are found in the literature that explores the LTC-HEV powertrain. Such powertrains are divided into two subcategories based on the LTC engine combustion regimes including the single-mode LTC and multi-mode LTC. In majority of the previous works, the engine has been flexible to switch from a single-mode LTC to a conventional mode [15, $\underline{34}, \underline{35}, \underline{36}]$. In the single-LTC mode subcategory, HCCI was the first type that was studied in electrified powertrains. In the first study at Argonne National Lab, the effects of using a dual-mode SI-HCCI engine in different vehicle electrification levels were analyzed [35]. In both studies, in references [34] and [36], the fuel economy benefits of the SI-HCCI engine are studied for parallel HEV architectures. RCCI was the second type of LTC engines that have been studied in an HEV powertrain. In reference [37], researchers at the University of WisconsinMadison and Oak Ridge National Laboratory used an RCCI engine in a series-parallel hybrid electric powertrain and they found $12 \%$ fuel economy improvement over the similar HEV running with a modern SI engine. In [15] the RCCI-CI engine is integrated in a power-split HEV architecture in which a rule-based energy management controller (EMC) was used for evaluating the fuel economy improvement. 


\subsection{Shortcomings of State-of-the-art Research}

Although hybridized LTC powertrain is promising to offer fuel economy advantage compared to conventional HEVs, the following shortcomings are found in the literature to properly assess the potential of LTC-HEV powertrains and address pertaining control challenges.

$\dagger$ Lack of any study for development of EMC strategies for LTC-HEV powertrains and also investigation into the effect of EMCs on the potential of fuel economy improvement for these powertrains,

$\dagger$ Lack of any study on purely dedicated LTC mode in LTC-HEV powertrain to investigate the ultimate powertrain fuel saving,

$\dagger$ Lack of any study on multi-mode LTC engine in hybrid electric powertrain to investigate the ultimate powertrain fuel saving,

$\dagger$ Lack of any study on the fuel consumption reduction of a multi-mode LTC-SI in a parallel HEV configuration by considering the emission constraints,

$\dagger$ Lack of any study on the effect of the hybridization level on the fuel saving over the single-mode SI in the parallel HEV,

$\dagger$ Lack of any study on the trade-off between the number of engine modes and the engine mode-switching fuel penalty on the powertrain fuel economy, 
$\dagger$ Lack of any experimental data for LTC-HEV models. This calls for design and building a test-bed to extract the powertrain components models.

\subsection{Objectives}

Four main objectives in this $\mathrm{PhD}$ dissertation research are:

1. Design, and build hybridized LTC test platform, with capability of the EMC strategies implementation, for extracting experimentally validated quasi-static models for model-based control of LTC-HEV powertrain in parallel HEV and SHEV architectures,

2. Develop EMC strategies including rule-based, global optimization, and model predictive control for hybrid powertrains integrated with LTC engines,

3. Analyze the effects of multi-mode LTC engine on the powertrain ultimate fuel economy of LTC-HEV powertrains,

4. Investigate effects of hybridization level, driving cycle type, and mode-switching fuel penalty on LTC-HEV powertrains.

This PhD research is addressed the shortcomings (Section 1.1) through the following chapters presented in this work: 
$\dagger$ Chapter 2: Investigate the fuel economy advantage of the single-mode HCCI engine in SHEV architectures compared to SI engines. Optimal EMC and MPC EMC strategies are developed and explained,

$\dagger$ Chapter 3: Investigate the fuel economy advantage of the single-mode RCCI engine in SHEV architectures, compared to SI and CI engines. Global optimal EMC and local optimum MPC EMC strategies are developed and explained,

$\dagger$ Chapter 4: Design, develop, and build a test setup for LTC-HEV powertrain experimental testing; build a control software platform for evaluation of modelbased EMC strategies. The models of the experimental setup are integrated with the optimization framework in the chapters 5 and 6 ,

$\dagger$ Chapter 5: Study the fuel saving and energy analysis of a multi-mode LTC-SI engine in SHEV architectures by including the mode-switching fuel penalty,

$\dagger$ Chapter 6: Study the fuel saving and energy analysis of the multi-mode LTC-SI engines in parallel HEV architectures for different hybridization levels.

Figure 1.2 depicts the organizations of this dissertation. 


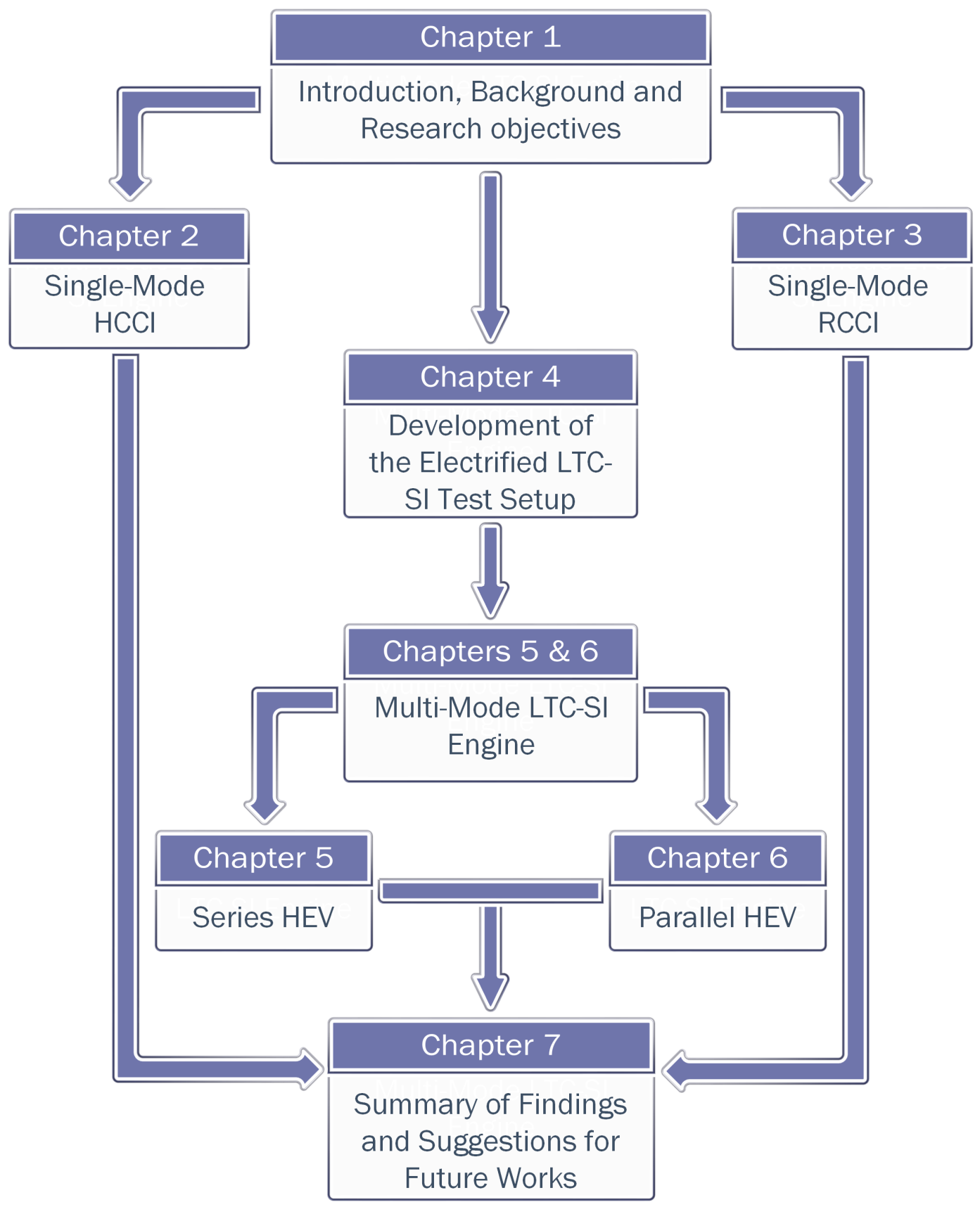

Figure 1.2: Structure of this $\mathrm{PhD}$ dissertation 



\section{Chapter 2}

\section{Single-Mode HCCI Engine as Range Extender 1}

Clean energy-efficient engine technologies including Low Temperature Combustion (LTC) show promise for fuel economy improvement in Hybrid Electric Vehicles (HEV). In this chapter, fuel economy improvement of a specific type of LTC engines, known as Homogeneous Charge Compression Ignition (HCCI), in synergy with a series Hybrid and Extended Range Electric Vehicle (E-REV) powertrain is investigated. An experimentally validated HCCI engine model is developed to simulate engine behavior. Three types of Energy Management Control (EMC) strategies are

\footnotetext{
${ }^{1}$ This chapter has been published in International Journal of Powertrain [1] with permission from Inderscience as shown in Appendix D
} 
designed and implemented. The EMC strategies encompass offline and online optimization strategies including thermostatic Rule-Based Controller (RBC), Dynamic Programing (DP), and Model Predictive Control (MPC). The simulation results are used to investigate the fuel economy benefits of an HCCI-based powertrain compared to a modern Spark Ignition (SI) engine-based powertrain in both series HEV and E-REV configurations. Moreover, the impact of number of engine operating points and driving cycles on the HCCI potential fuel economy improvement are examined. The results show $17.7 \%$ fuel economy improvement in Series HEV and $18.0 \%$ fuel economy improvement in E-REV compared to a conventional HEV using an SI engine in Urban Dynamometer Driving Schedule (UDDS) driving cycle. In addition, simulation results show the HCCI fuel economy improvement of $16.2 \%$ in New European Driving Cycle (NEDC) and $18.9 \%$ in JC08 Japanese driving cycles. Simulation results show that selection of the number of engine operating points accounts for up to $10 \%$ difference in fuel economy improvement. DP-based EMC provides $15.6 \%$ fuel economy advantage over the thermostatic RBC in an HEV using an HCCI engine.

\section{$2.1 \quad$ Introduction}

The U.S. transportation sector will account for 28 percent of total U.S. energy usage by 2040 [38]. In contrast to the historical trend of 1.1 percent energy growth in the average annual transportation sector from 1975 to 2011 [39], this number will remain 
almost identical to the current value by 2040. This lack of growth is due to the projected reduction in energy use for Light Duty Vehicles (LDVs), which compensates for energy consumption increase in other transportation sectors such as aircraft, marine, and rail even though the Vehicle Miles Travel (VMT) projection increases almost one thousand miles per vehicle by 2040. This reduction in LVDs energy usage is a result of the projected improvement in the fleet fuel economy. This significant improvement in LVDs is not reachable by simply improving conventional powertrains, thus the integration of high efficient Internal Combustion Engines (ICEs) and vehicle electrification (i.e., HEVs) shows the most promise [34]. This chapter investigates fuel economy benefits of fuel-efficient ICE technology (i.e., LTC engines) in synergy with an HEV.

The projected statistics of HEV and Electric Vehicle (EV) sales show a remarkable growth by 2040 to meet the more restricted CAFE and Greenhouse Gas standards [39]. The greatest growth in sales value belongs to vehicles with greater All Electric Range (AER) including high range Plug-in HEVs (PHEVs) and EVs, which will increase by $91.2 \%$ and $13.3 \%$ from 2011 to 2040 , respectively, compared to only $3.5 \%$ sale increase for Conventional HEVs in 2040 [39]. Moreover, EVs can be designed as ExtendedRange Electric Vehicles (E-REVs) to alleviate range anxiety concerns by increasing the vehicle AER. In Series HEV architecture and range extender, the ICE is used as a genset to boost the driving range of the car. There are currently some vehicles on the market that use this concept, like the Chevrolet Volt, Fisker Karma, BMWi3, 
and Cadillac ELR, and their number is growing. The focus of this chapter is on a Series HEV and an E-REV.

Furthermore, the largest potential fuel economy improvement in HEVs and E-REVs is provided by improvement in ICE fuel efficiency, compared to improvement in other components of HEVs [40]. An LTC engine encompasses a family of ICE technologies, including Premixed Charge Compression Ignition (PCCI), Homogenous Charge Compression Ignition (HCCI), and Reactivity Controlled Compression Ignition (RCCI) engines. In this chapter, the focus is on utilizing HCCI in a Series HEV and E-REV. HCCI technology combines the benefits of both Spark Ignition (SI) and Compression Ignition (CI) engines. In addition, HCCI offers peak indicated thermal efficiency up to $50 \%$ [41] which is higher than SI and CI engines, while having low Nitrogen Oxides (NOx), and negligible Particulate Matter (PM) emissions [16]. The drawback of an HCCI engine is its narrow operating range, which can be tackled by using a Series HEV architecture or range extender without the need to switch the engine operating mode. In this chapter, the potential fuel saving of using an HCCI engine in series HEV and E-REV configurations is compared to the same configurations running by a modern SI engine.

Previous studies on different types of ICE utilization in HEVs are divided into three main groups: CI engines, SI engines, and LTC engines. Figure 2.1 outlines some of the major ICE types along with the different types of EMCs used in HEVs. In the 
first category, a CI engine has been used in different HEV architectures [42, 43, 44, 45, 46, 47, 48, 49]. The CI engines have been mostly used in Sport Utility Vehicles (SUVs) and trucks [44, 46, 48, 48, 50]. In another category, SI engines have been used in HEV configurations, and E-REV [51, 52, 53, 54]. Recently, Atkinson cycle-based engines have become more popular in the market (e.g. Ford C-Max, Toyota Prius, Lexus RX 450h, and Honda Accord PHEV). In [28] Toyota R\&D group redesigned an engine based on the Atkinson cycle to improve the engine fuel efficiency. In addition, in [27], a Honda Accord PHEV was redesigned based on the Atkinson cycle and 10\% lower fuel consumption was achieved compared to the SI engine. In the literature, some studies conducted on integrating LTC engines with HEVs. In the first study at Argonne National Lab, the effects of using a dual mode SI-HCCI engine in different ranges of vehicle electrification levels were studied [35]. They predicted a range of $6 \%$ to $15 \%$ fuel consumption reduction in different powertrains and driving cycles. In another study [34], fuel economy improvement of an HCCI engine versus an SI engine for two mild and medium parallel HEVs were done. The authors could achieve a range from $17 \%$ to $35 \%$ fuel economy improvement by using a dual-mode SI-HCCI in comparison to a conventional SI non-hybrid powertrain. In both [34, 35] studies, a dual-mode (SI-HCCI) engine is used with a rule-based EMC. This thesis includes the first study on pure HCCI mode engine synergy with a series hybrid powertrain. This chapter investigates the impact of driving cycles, number of the engine operating points, and engine startup fuel penalty on both series HEV and E-REV powertrains. 


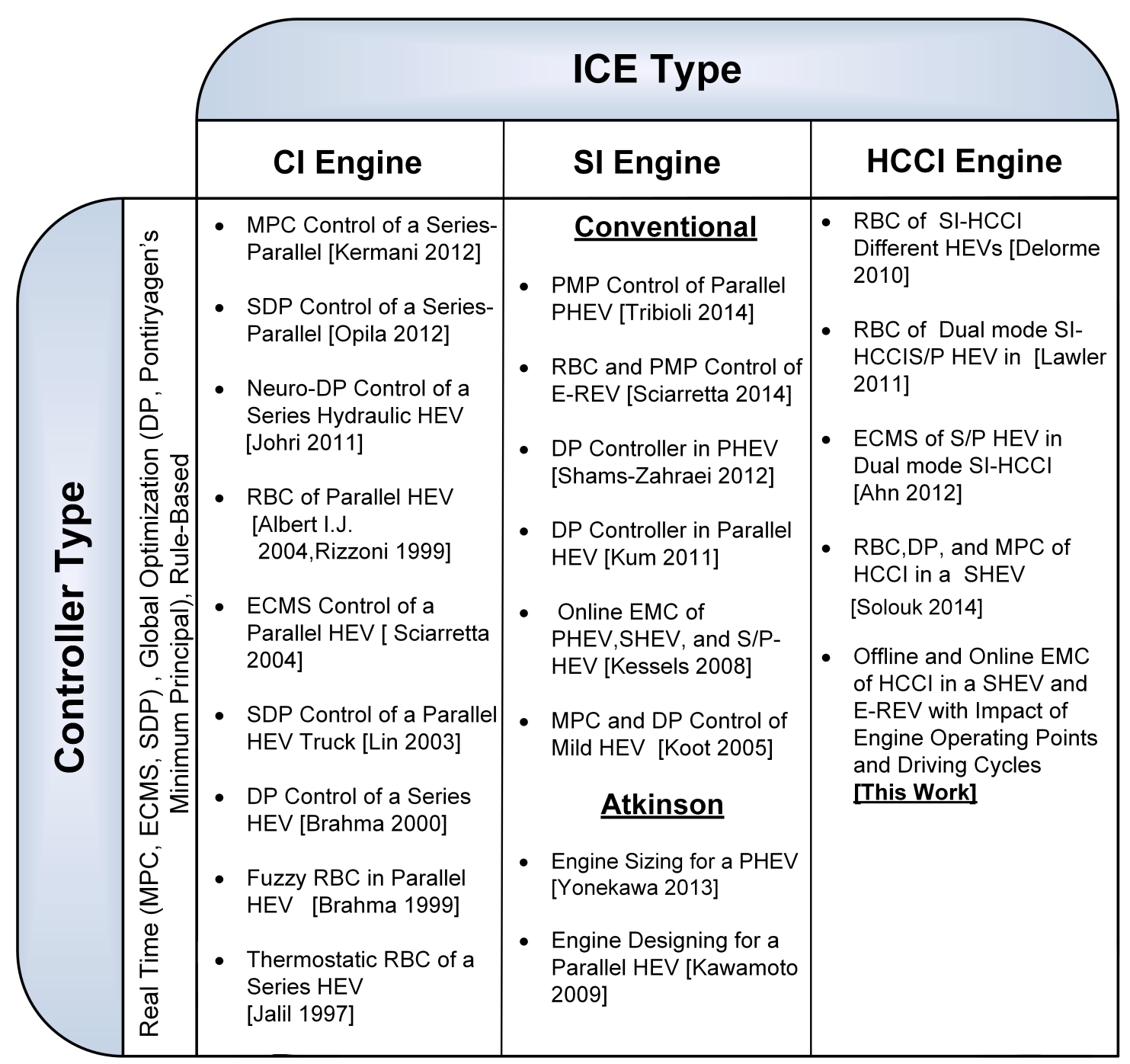

Figure 2.1: Different types of ICEs and EMCs used in HEVs in previous studies.

In addition, Figure 2.1 lists the different EMC approaches in HEV control. These include 1) Rule-Based Controller (RBC) such as thermostatic [45] and fuzzy [50] strategies. The RBC strategies are robust and have low computational costs. However, they offer only limited optimization and may fail to completely exploit the HEV 
benefits [55, 56]; 2) Offline global optimization such as Dynamic Programming (DP) [49, 57], and Pontryagin Minimum Principle (PMP) [51, 52]; the DP method is a global optimization method that assumes the information of an entire driving cycle is available and numerically finds the global optimal solution. This method is not implementable in real applications due to the necessity of knowing a priori of the driving cycle [55]; 3) Real time optimization controllers such as Model Predictive Controllers (MPC) [42, 58], Equivalent Consumption Minimization Strategy (ECMS) [47, 59], and Stochastic Dynamic Programming (SDP) [48]. The MPC method is a local optimization method that is implementable online but requires high computation. In this chapter, three different EMCs, including a representative from each group, are considered to determine the impacts of an HCCI engine in a series HEV and an E-REV.

To the best of the author' knowledge, this is the first study undertaken to analyze hybridization of an LTC engine in a series HEV and E-REV configuration. The contribution from this chapter is threefold. First, it investigates the potential fuel economy improvement by using a dedicated HCCI mode in a series HEV and an E-REV. Second, it shows the first implementation of different EMC strategies for a series HEV architecture integrated with an HCCI engine. Third, it studies the impact of the number of engine operating points on the fuel economy of HCCI-based HEV.

This chapter is organized as follows. In Section 2.2, the developed HEV model is 
presented and the main components of the HEV model are described. Section 2.3 explains three different EMC strategies including thermostatic RBC, DP, and MPC. The Model validation results are shown in Section 2.4. In addition, the results of the series HEV with single engine operating point are discussed in Section 2.4.2. The effect of the number of engine operating points on the series HEV fuel economy improvement is presented in Section 2.4.3. Besides the series HEV, another study is done for E-REV and the results of the fuel economy are reported in the Section 2.4.4. Moreover, the effect of the driving cycle on the both series HEV and E-REV fuel economy improvement is presented in Section 2.4.5. Finally, Section 2.5 summarizes all findings from this chapter.

\subsection{HEV Modeling}

A nonlinear and forward-in-power HEV model is developed in Matlab/Simulink to evaluate the EMC strategies and fuel economy benefits. Subsequently, the series HEV model was built by integrating submodels for the HCCI engine, generator, E-motor, battery pack, and Longitudinal Vehicle Dynamic (LVD). The supervisory control module was introduced within the powertrain to manage the balance between the multiple power sources onboard. The top level Simulink model of the series $\mathrm{HEV}$ is shown in Figure 2.2, where the main focus is on HCCI engine synergy with the main HEV model components and the supervisory controller. A PID controller 
simulates the driver behavior to track the target driving cycle. Based on the driverrequested power and the battery State Of Charge (SOC), the supervisory controller specifies the engine power profile and the battery pack power request. The E-motor power request is supplied by the battery pack and the engine/generator. The LVD submodel includes accurate aerodynamic and rolling resistance forces for a typical sedan. The HCCI engine and generator submodels are quasi-static models. In the following sections, detailed models of the major HEV components are presented. The description for sizing of each HEV component is provided in the Appendix A.

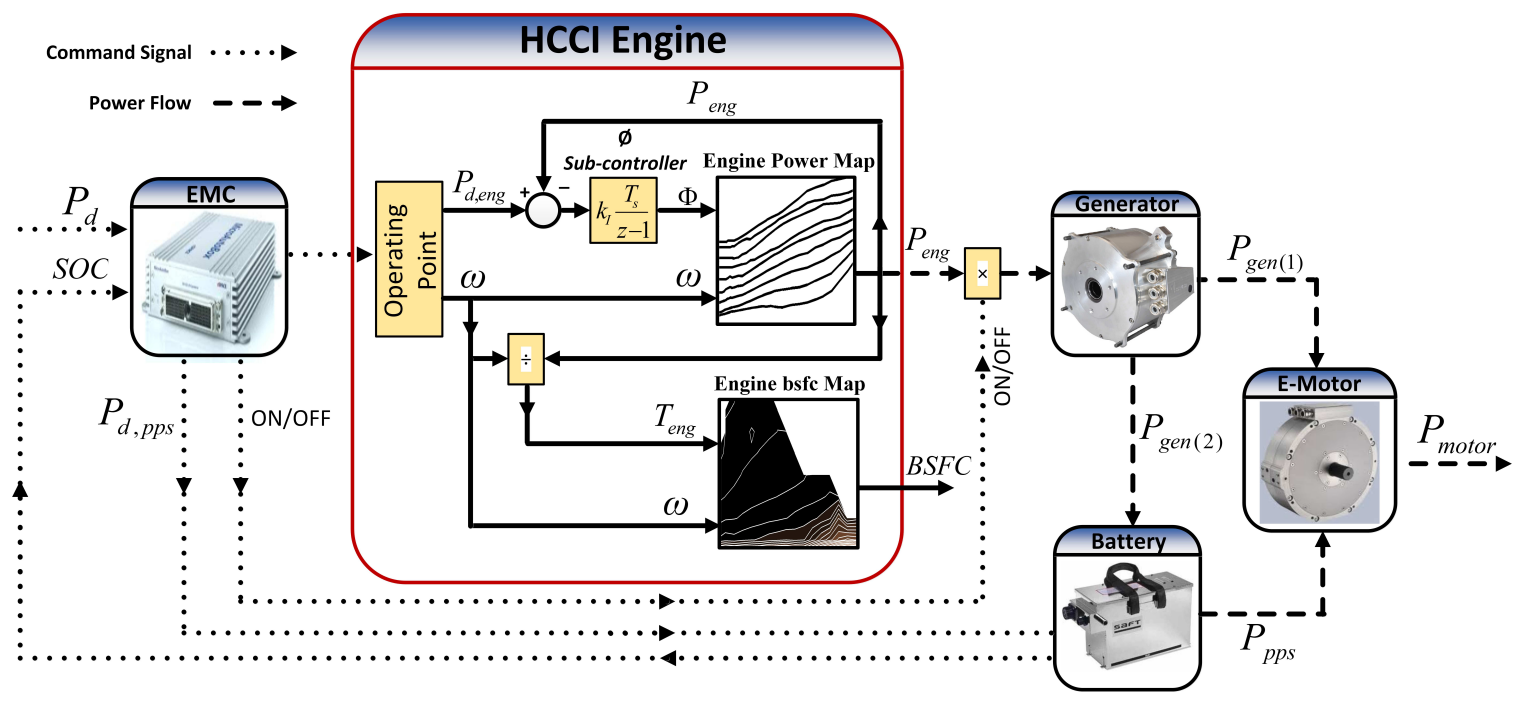

Figure 2.2: A schematic diagram to illustrate the power and control flow and main components of a series HEV.

\subsubsection{HCCI Engine}

Table 2.1 lists the HCCI engine specifications. The rated produced power of the engine is $24.1 \mathrm{~kW}$. The engine speed range is from $800 \mathrm{rpm}$ to $2500 \mathrm{rpm}$ with IMEP 
varying from 3.6 to 9.1 bar [60]. This engine operates under ultra-lean and lean conditions $(0.38<\phi<0.72)$ as shown in Table 2.1. A detailed physical HCCI engine model is found in [20] is used in this chapter. The HCCI model has been extensively validated against steady-state and transient experimental data in [20, 61].

Table 2.1

HCCI engine parameters.

\begin{tabular}{lc}
\hline \hline Parameters & Values \\
\hline Max. Power (kW) @ 2500 rpm & 24.1 \\
Max. Torque (N.m) @ $1300 \mathrm{rpm}$ & 160.6 \\
Engine Speed (rpm) & $800-2500$ \\
Fuel-Air Equivalence Ratio, $\phi(-)$ & $0.38-0.72$ \\
Intake Pressure (kPa) & 135 \\
\hline \hline
\end{tabular}

The input to the HCCI engine model is the fuel equivalence ratio $(\phi)$; and the output is power and fuel consumption based on the engine power needed. To calculate the generated power and fuel consumption of the HCCI engine, the $\phi$ value must be known. Thus, a PID controller is designed to determine the required $\phi$ values for different levels of power needed from the HCCI engine. One engine map is designed to relate $\phi$ and speed to the generated power (Figure [2.3-a). The engine output torque is known by dividing the engine output power by the engine speed, so another engine map is developed to relate the engine torque and speed to Brake Specific Fuel Consumption (BSFC) to calculate the fuel consumption. The resulting BSFC map is shown in Figure 2.3.b. 
(a)

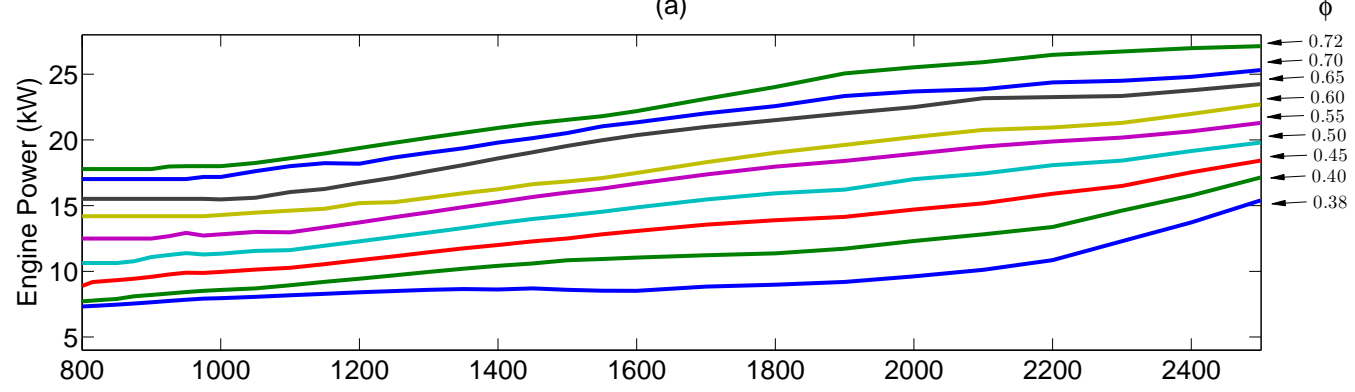

(b)

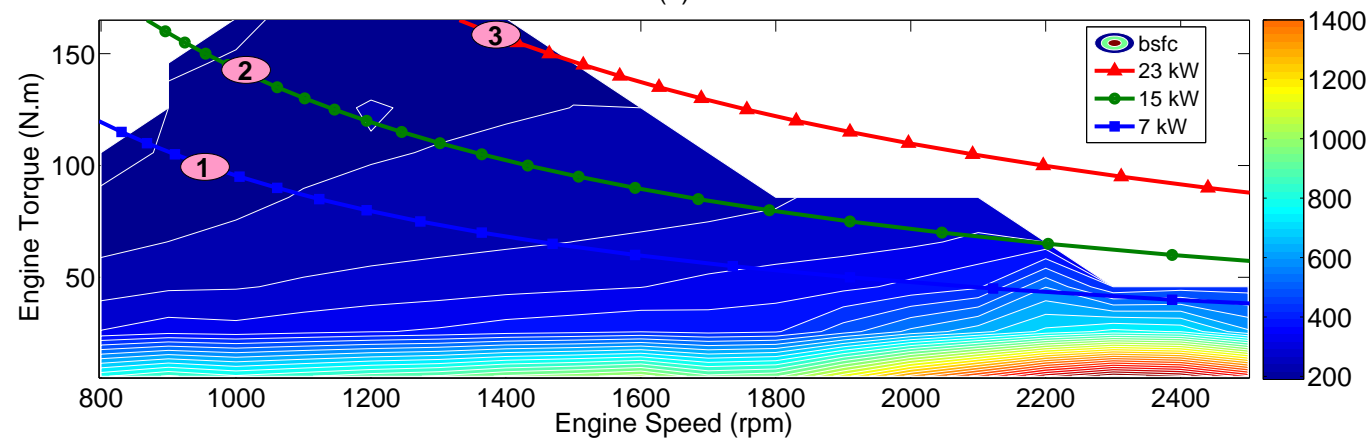

Figure 2.3: HCCI engine power and BSFC variation as a function of engine speed and $\phi$.

\subsubsection{Battery}

A battery model from [62] is used to calculate the SOC based on the battery power usage. This model has been used widely in previous EV and HEV studies due to its simple implementation [63]. One output of the supervisory EMC in Figure 2.2 is the battery requested power, which is used by the battery model to calculate the SOC and the battery voltage. The battery model assumes identical charging and discharging characteristics for the battery. The battery open circuit voltage $(E)$ has a nonlinear relationship with the actual battery current $(i)$, which is introduced by: 


$$
E=E_{0}-K \frac{Q \cdot i}{Q-\int i d t}+A \exp \left(-B \cdot \int i d t\right)
$$

where $Q$ is the battery nominal capacity. $A, B$, and $K$ are the battery parameters obtained from the battery voltage discharge curve. The values for these parameters are provided in the Appendix $\mathrm{A}$. The $S O C$ is calculated by:

$$
S O C=1-\frac{\int i d t}{Q}
$$

The battery energy capacity in this chapter was designed to supply the vehiclerequired electrical energy for 40 miles AER, which is in the same range as other E-REVs, including the Chevy Volt and Cadillac ELR. Energy capacity is calculated based on the 300Wh/mile energy requirement of a mid-size passenger car [64] as the energy capacity highly depends on the driving cycle. Thus, the battery pack energy capacity in this chapter is sized to address this requirement. Table 2.2 shows the specifications of the selected battery. More discussion on the battery sizing is found in the Appendix $\mathrm{A}$. 
Table 2.2

LV30P SAFT Li-ion battery parameters.

\begin{tabular}{lc}
\hline \hline Parameters & Values \\
\hline Max Power $(\mathrm{kW})$ & 215 \\
Capacity (kwh) & 18.5 \\
Mass (kg) & 190 \\
Specific Power $(\mathrm{W} / \mathrm{kg})$ & 1136 \\
Specific Energy $(\mathrm{Wh} / \mathrm{kg})$ & 97 \\
Max. Peak Power $(\mathrm{A})$ & 500 \\
\hline \hline
\end{tabular}

\subsubsection{Traction E-motor}

The actual produced power of the E-motor is obtained from the efficiency map at each power-speed operating point. The map covers both motoring and braking modes. Equation (2.3) shows the torque relation of the E-motor:

$$
T_{\text {motor }}= \begin{cases}\frac{P_{\text {available }} \eta_{\text {motoring }}}{\omega_{\text {motor }}} & \text { (a) motoring } \\ \frac{P_{\text {available }}}{\omega_{\text {motor }} \eta_{\text {braking }}} & \text { (b) braking }\end{cases}
$$

where $P_{\text {available }}$ refers to the available electrical power in the battery. Consequently, the vehicle traction force $\left(F_{\text {traction }}\right)$ and the E-motor speed $\left(\omega_{\text {motor }}\right)$ are calculated by: 


$$
\begin{aligned}
& F_{\text {traction }}=\frac{T_{\text {motor }}}{R} G r \\
& \omega_{\text {motor }}=\frac{V_{\text {vehicle }}}{R} G r
\end{aligned}
$$

where $G r$ is the final gear ratio between the E-motor and the driveline, and $R$ is the wheels radius. The actual vehicle speed $\left(V_{\text {vehicle }}\right)$ is obtained from the LVD model, which will be presented in the section 2.2.4. The UQM E-motor is chosen among different common HEV traction E-motors based on the vehicle's gradeability and acceleration constraints (see the Appendix $\mathrm{A}$ for details). Table 2.3 lists the specifications of the UQM E-motor.

Table 2.3

Parameters for UQM PowerPhase 75 traction E-motor model.

\begin{tabular}{lc}
\hline \hline Parameters & Values \\
\hline Peak Power (kW) & 75 \\
Peak Torque (N.m) & 240 \\
Maximum Speed (rpm) & 7500 \\
Maximum Efficiency & 0.94 \\
Voltage Range (V) & $250-400$ \\
Peak Input Current (A) & 400 \\
Gear Ratio & 2 \\
Mass (kg) & 41 \\
\hline \hline
\end{tabular}




\subsubsection{LVD Model}

The purpose of the LVD model is to calculate the vehicle actual speed $(V)$ based on vehicle dynamics:

$$
M \frac{d V}{d t}=F_{\text {traction }}-F_{\text {drag }}-F_{\text {roll }}-F_{\text {gravity }}
$$

where $M$ is the vehicle total mass; $F_{\text {drag }}, F_{\text {roll }}$, and $F_{\text {gravity }}$ are aerodynamic drag force, rolling resistance force and gravity force, respectively. Each of these parasitic forces are calculated by:

$$
\begin{gathered}
F_{\text {drag }}=\frac{1}{2} \rho A C_{d} V^{2} \\
F_{\text {roll }}=M g f_{r}\left(1+\frac{V}{100}\right) \\
F_{\text {gravity }}=M g \cos \theta
\end{gathered}
$$

where $\rho$ is the air density, $A$ is the vehicle frontal area, $C_{d}$ is the vehicle aerodynamic drag coefficient, $f_{r}$ is the rolling resistance coefficient, and $\theta$ is the road slope. Table 2.4 lists parameters of the LVD model. 
Table 2.4

Vehicle specifications.

\begin{tabular}{lc}
\hline \hline Parameters & Values \\
\hline Vehicle Curb Weight (kg) & 1411 \\
Differential Gear Ratio & 3.25 \\
Wheel Radius (m) & 0.381 \\
Drag Coefficient & 0.25 \\
Rolling Resistance & 0.01 \\
\hline \hline
\end{tabular}

\subsubsection{Vehicle Performance}

The HEV model is implemented in Matlab/Simulink. The vehicle acceleration performance was designed to reach $60 \mathrm{mph}$ in 12.2 seconds, which is similar to a common mid-size HEV on the market [65]. The vehicle braking performance was designed to stop from $60 \mathrm{mph}$ by traveling 53.4 meters [65]. Figure 2.4 shows acceleration and braking performance of the developed HEV model. The model was verified with the performance target values, which include a 0 to $60 \mathrm{mph}$ acceleration time of $12.2 \mathrm{sec}-$ onds and braking distance of 47 meters when stopping from $60 \mathrm{mph}$. These numbers verify that the developed HEV model has similar acceleration and better braking performance than a common mid-size HEV. In addition, the model can satisfy the gradeability requirements for a mid-size HEV (see the Appendix A). 


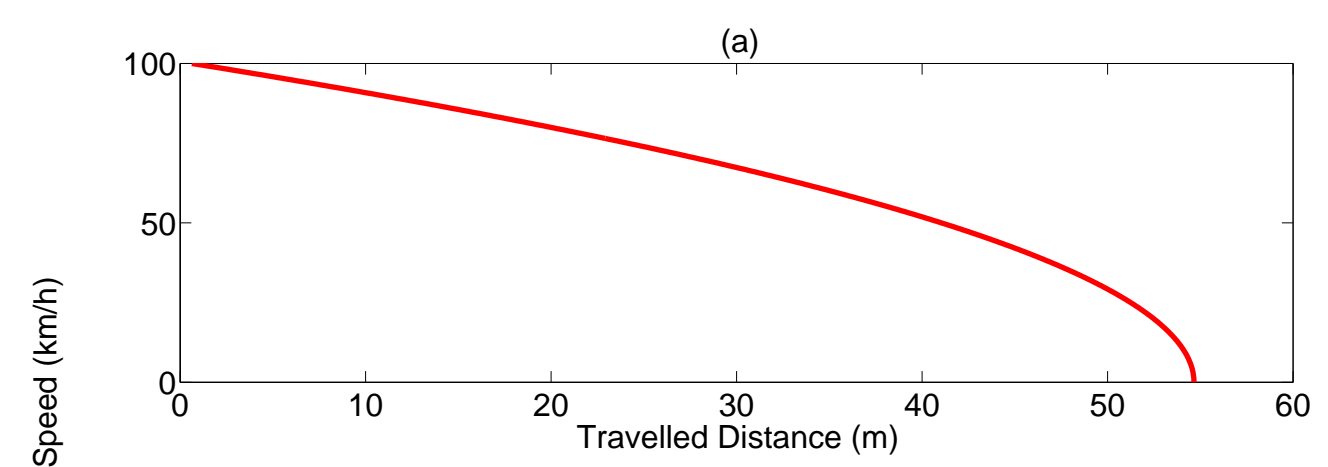

(b)

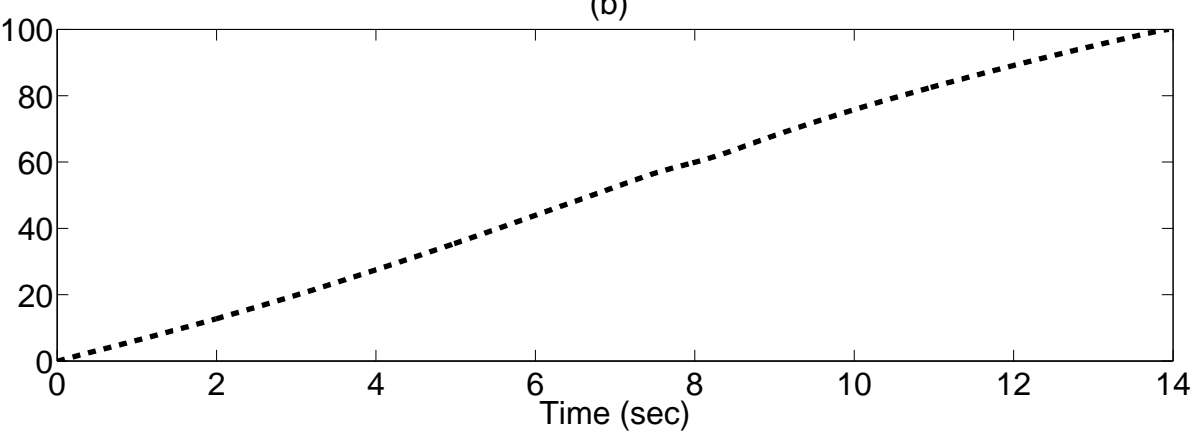

Figure 2.4: Simulated acceleration and braking performance of the HEV in this chapter.

\subsection{Energy Management Controller (EMC) De-}

\section{$\operatorname{sign}$}

The most important objectives of the EMC strategies include $(i)$ meeting the driver requested power for the traction, (ii) sustaining the battery charge, and (iii) minimizing fuel consumption. Here, three types of EMC strategies are developed and tested for the HEV with the HCCI engine. These strategies include thermostatic RBC control, global optimal control (i.e., DP), and MPC. Desired SOC window of $0.5 \leqslant S O C \leqslant 0.9$ is used in all the three EMCs. 


\subsubsection{EMC Type I: Rule-Based Control (RBC)}

In thermostatic RBC, the EMC rules are designed heuristically without driving cycle information. The battery SOC is the only input to the control unit, which forces the battery to keep the SOC on the desired window $(0.5 \leqslant S O C \leqslant 0.9)$, by controlling the engine on/off status. The thermostatic RBCs cannot adapt their rules with the driving cycles, which results in non-optimal efficiency for a wide operating range. Simple implementation for real-time EMC application is the major advantage of the thermostatic RBC strategies [66].

\subsubsection{EMC Type II: Global Optimization - Dynamic Pro- gramming (DP)}

In a series $\mathrm{HEV}$, the engine power profile is determined by optimization-based EMC while minimizing a cost function. If the driving cycle is known, meaning that the driver power request is known, then a global optimal solution can be found [66]. While this approach cannot be applied in real-time EMC applications, the solution from this method can be used as an ideal reference set for evaluating other optimal EMCs. In this chapter, DP method based on Bellman's Principle of Optimality [67] is used to find a global optimum solution assuming that the entire driving cycle is 
known in advance. To design the EMC strategy, first a simple backward model of the series HEV is used to decrease the optimization problem calculation time [49, 68]. This model simplifies the whole series HEV in two paths including fuel energy path and battery energy path. The backward model replaces the intermediate components (i.e. engine, generator, and battery) in the path by their net effect on the combined path efficiency.

The transition cost between $k^{\text {th }}$ state to $j^{\text {th }}$ state at successive times is defined by $J_{j}\left(t, u_{k}\right)$ from the following equation:

$$
J_{j}\left(t, u_{k}\right)=\underset{j}{\Delta E_{f}}\left(t, u_{k}\right)+\alpha \underset{j}{\Delta E_{b}}\left(t, u_{k}\right)
$$

The index $j$ represents the feasible transitions between two states in the successive times and the index $k$ shows the control state at the current time $t$. Furthermore, $\underset{j}{\Delta E_{f}}\left(t, u_{k}\right)$ and $\underset{j}{\Delta E_{b}}\left(t, u_{k}\right)$ are the energy consumptions of the ICE path and the battery path respectively for the transition between the $k^{\text {th }}$ state to the $j^{\text {th }}$ state in the successive times. The control variable, $u_{k}$, is the required ICE/generator power produced which is determined by the DP control policy. The battery energy is weighted by an equivalence factor $\alpha$. If $\alpha$ is large, the battery path will be in a charge sustaining mode. In contrast, if $\alpha$ is small, then the battery energy increases, which results in SOC depletion. In this chapter, $\alpha$ is a constant value that is tuned by an offline optimization to enforce the battery to operate in a charge sustaining 
mode. By changing the $\alpha$ value, the battery can operate in a charge depleting mode.

$$
\begin{gathered}
\underset{j}{\Delta E_{f}}\left(t, u_{k}\right) \text { and } \underset{j}{\Delta E_{b}}\left(t, u_{k}\right) \text { are calculated by the following equations: } \\
\underset{j}{\Delta E_{f}\left(t, u_{k}\right)=} \frac{1}{2}\left(\left[\frac{P_{\text {fpath }}\left(t, u_{k}\right)}{\eta_{\text {fpath }}\left(t, u_{k}\right)}+\frac{P_{\text {fpath }}(t+1, j)}{\eta_{\text {fpath }}(t+1, j)}\right] \Delta t+N_{k j} m_{f, \text { start }} Q_{L H V}\right) \\
\Delta E_{j}\left(t, u_{k}\right)=\frac{1}{2}\left[\frac{P_{\text {bpath }}\left(t, u_{k}\right)}{\eta_{\text {bpath }}\left(t, u_{k}\right)}+\frac{P_{\text {bpath }}(t+1, j)}{\eta_{\text {bpath }}(t+1, j)}\right] \Delta t
\end{gathered}
$$

where $\eta_{\text {fpath }}$ and $\eta_{\text {bpath }}$ represent the combined efficiency in the ICE path and the battery path for the transition between the $k^{\text {th }}$ state to the $j^{\text {th }}$ state in the successive times, respectively. $m_{f, s t a r t}$ in Equation (2.11) is the fuel penalty for each engine start-up. The cost associated with the engine start-up is incorporated in Equation (2.11) by introducing the $N_{k j}$ constant which is equal to 1 when engine is at start-up and it is equal to 0 during the rest of the engine operation. $Q_{L H V}$ is the gasoline fuel lower heating value.

The summation of produced power from the fuel path and the battery path should be equal to the driver requested power $\left(P_{r e q}\right)$. Thus, in the DP strategy, by specifying the required engine power $\left(P_{\text {fpath }}\left(t, u_{k}\right)\right)$ at the $k^{t h}$ state, the required battery power $\left(P_{\text {bpath }}\left(t, u_{k}\right)\right)$ at the same state in time $t$ can be calculated by:

$$
P_{\text {bpath }}\left(t, u_{k}\right)=P_{r e q}\left(t, u_{k}\right)-P_{\text {fpath }}\left(t, u_{k}\right)
$$


The discretization of the DP problem is done with 1 second time step over the whole driving cycle period. In the backward DP, the optimal cost-to-go from the current time $(t)$ to the end of the driving cycle is defined as:

$$
S^{*}\left(t, u_{k}\right)=\min _{j}\left[J_{j}\left(t, u_{k}\right)+S^{*}(t+1, j)\right]
$$

where $S^{*}\left(t, u_{k}\right)$ is the optimal cost-to-go from the $k^{\text {th }}$ state at the current time $t$ to the end of the driving cycle. $S\left(t+1, u_{j}\right)$ represents the optimal cost in the next time (i.e., $t+1$ ) associated with the control state $u_{j}$ to the end of the driving cycle.

A nonlinear HEV model, which does not include the driver model, is used to increase the real-time implementation flexibility of optimal EMC model. This backward model assumes that the vehicle tracks exactly the driving cycle; thus, the vehicle power demand directly calculates from the driving cycle. In addition, a complex forward Simulink vehicle model is designed for analysis of the HEV vehicle. For confirming that the backward model can track dynamics of the target HEV, simulation results of the backward model are compared to those of the complex forward Simulink vehicle model. The comparison results in Figure 2.5 show that the calculated E-motor power request from the backward model is close to the complex forward model. Therefore, the backward model can be used as a plant model for DP controller design, while 
the final EMC strategies are tested in the detailed forward HEV model. It should be noted that implementing the DP for real-time operation is challenging. The purpose for including DP results in this chapter is to present the ultimate energy saving using the HCCI hybrid electric powertrain. The DP values serve as a benchmark for the comparison with $\mathrm{RBC}$ and $\mathrm{MPC}$ results with different time horizons.

(a)

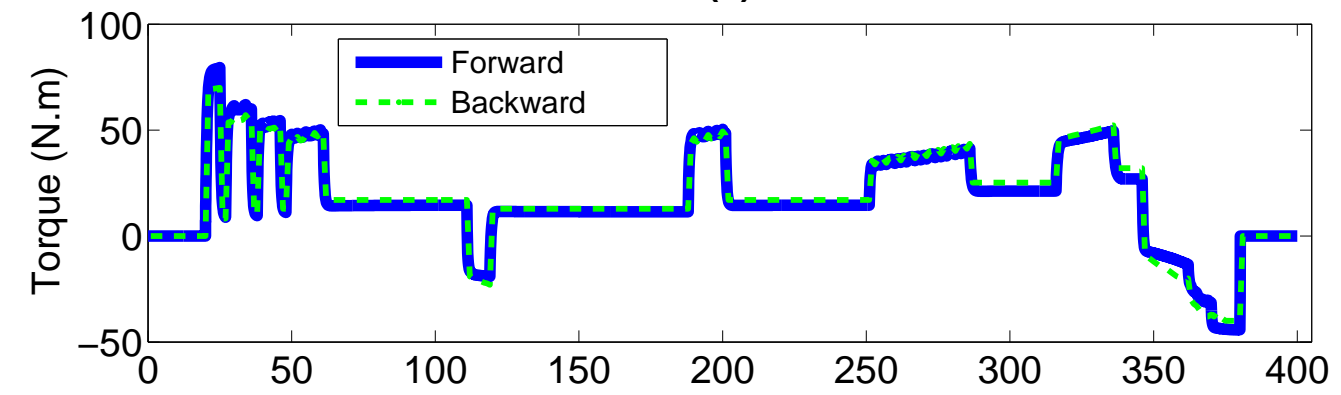

(b)

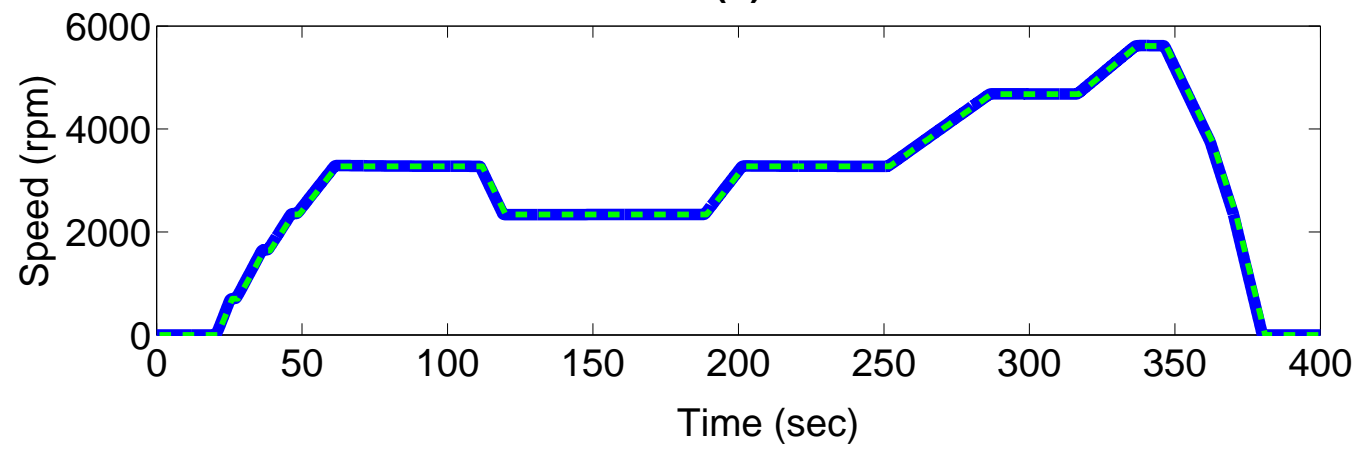

Figure 2.5: Comparison of E-motor speed and torque simulation results from forward and backward HEV models during Extra-Urban Driving Cycle (EUDC) driving cycle. 


\subsubsection{EMC Type III: Model Predictive Controller}

The majority of the EMC optimization techniques require the information of the entire driving cycle in advance. This requirement makes it challenging to implement EMC strategies in real-time [69]. Given that the vehicle speed can be predicted over a short Time Horizon (TH), the optimization method can be applied over a short $\mathrm{TH}$ instead of over the entire driving cycle. The vehicle velocity information over a short TH can be obtained from the GPS estimated data. The cost function $(J)$ in the $n^{\text {th }} \mathrm{TH}$ is shown in Equation (2.15). $J$ is minimized over TH by selecting optimal ICE/generator power.

$$
J(n)=\int_{t_{n}}^{t_{n}+T H}\left(P_{\text {fpath }}+\alpha \cdot P_{b p a t h}\right) d t
$$

subject to: 


$$
\begin{array}{r}
P_{e, \text { min }}<P_{e}<P_{e, \text { max }} \\
P_{m, \text { min }}<P_{m}<P_{m, \max } \\
N_{e, \min }<N_{e}<N_{e, \max } \\
N_{m, \min }<N_{m}<N_{m, \max } \\
S O C_{\min }<S O C<S O C_{\max } \\
S O C_{@ t=0}=0.8
\end{array}
$$

where the subscripts min and max denote minimum and maximum, respectively. The first term in Equation (2.15) refers to the fuel energy consumed by the ICE and the second term refers to the battery electrical energy consumed or recharged during the drive cycle. The subscripts $e$ and $m$ in Equation (2.16) designate engine and motor, respectively. The DP formulation in Section 2.3.2 is used over the $\mathrm{TH}$ to calculate the optimal ICE/generator power at time step $n$. A closed-loop MPC is designed to reject disturbances such as sudden changes in the estimated driving cycle data. However, this EMC strategy does not provide a globally optimal solution, but it can be used for real-time implementation. In the current formulation, the time domain is discretized into one-second intervals. The solutions consist of local optimum control signal at each time step. 


\subsection{Results and Discussions}

In this section, firstly, validation of the developed model components is shown; secondly, the results for the single model series HEV is presented and then the impact of the engine operating points and the results for the E-REV model are discussed. Lastly, the effect of driving cycles type on the fuel economy is examined. In subsections 2.4.2 to 2.4.4 Urban Dynamometer Driving Schedule (UDDS) driving cycle is used. The impact of the engine operating points and the type driving cycles are presented in the subsections 2.4 .3 and 2.4.5, respectively.

\subsubsection{Validation of Model Components}

The HCCI engine model has been validated previously against the experimental data at a wide operating range [20]. Here validation of two major HEV components, including the E-motor and battery, is presented.

The battery model accuracy is verified by comparing the battery model single cell discharge profile with the one obtained from experimental data [5]. Figure 2.6 shows that the model can predict the battery discharge performance by Root Mean Square Error (RMSE) of $0.17 \mathrm{~V}$. 


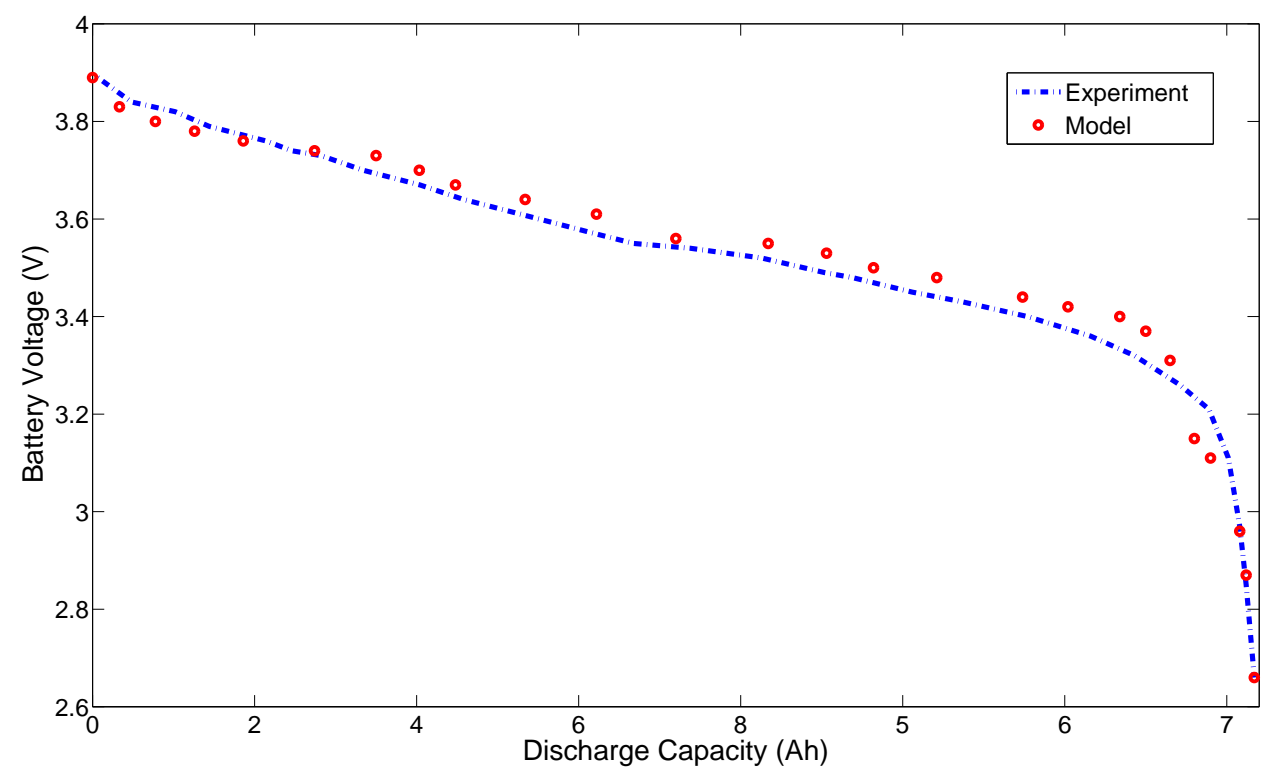

Figure 2.6: Battery model validation against experimental data from [5].

In addition, Figure 2.7 compares the E-motor model accuracy at different operating points. The comparison is done for both motoring and regeneration modes. Validation results show that the E-motor model can predict efficiency of the E-motor for both motoring and regeneration conditions with RMSE less than $1.5 \%$.

\subsubsection{Single Mode Series HEV}

In series HEVs, the engine is decoupled from the drivetrain, which makes the engine work most of the time at high efficiency operating points. Here, the engine is working on an operating point to produce $15 \mathrm{~kW}$. This amount of power is enough for the engine to keep the vehicle speed constant at $70 \mathrm{mph}$ on a flat road (see Appendix A). 


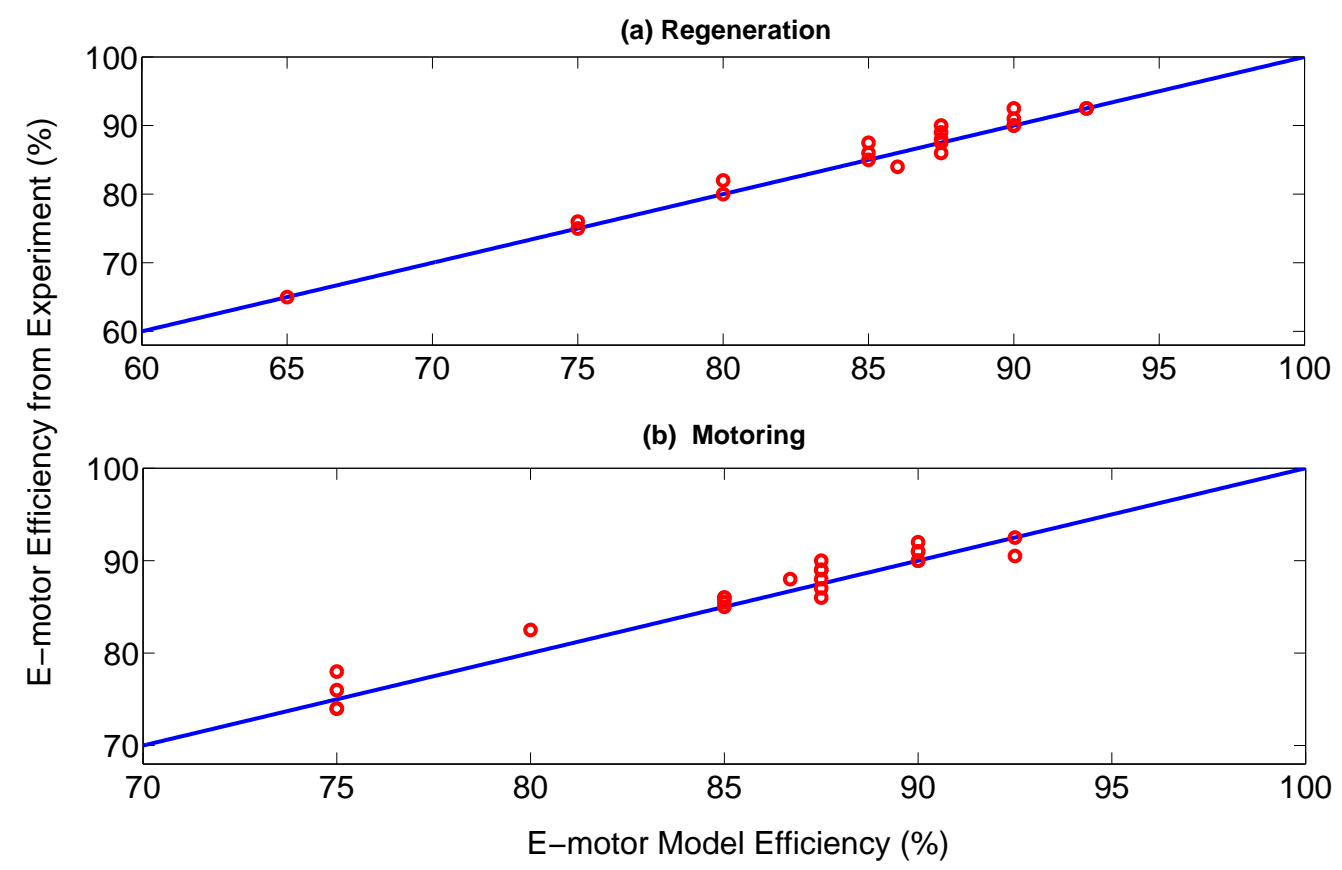

Figure 2.7: Experimental validation of E-motor efficiency using experimental data from [6].

From Figure 2.3 the minimum BSFC of the HCCI engine at $15 \mathrm{~kW}$ power occurs at $900 \mathrm{rpm}$ engine speed and $160 \mathrm{~N}-\mathrm{m}$ torque (point 2 on Figure 2.3-b). This operating point is used for design of EMCs in this chapter. The rest of the power request will be produced by the battery pack; thus, the power from the battery keeps changing during the driving cycle.

To evaluate the HCCI engine fuel economy, a modern SI engine model is developed and integrated into the HEV model in this chapter. The selected engine is a GM Ecotec 1.4L 4-cylinder SI engine, which is used in the Volt E-REV. Figure 2.8 presents the GM engine BSFC map based on the data from [7]. For $15 \mathrm{~kW}$, the optimum BSFC is $245 \frac{g}{k W h}$ at $1500 \mathrm{rpm}$ with $95.5 \mathrm{~N}$.m engine torque. This single operating point is 
used as a basis of comparison in this section.

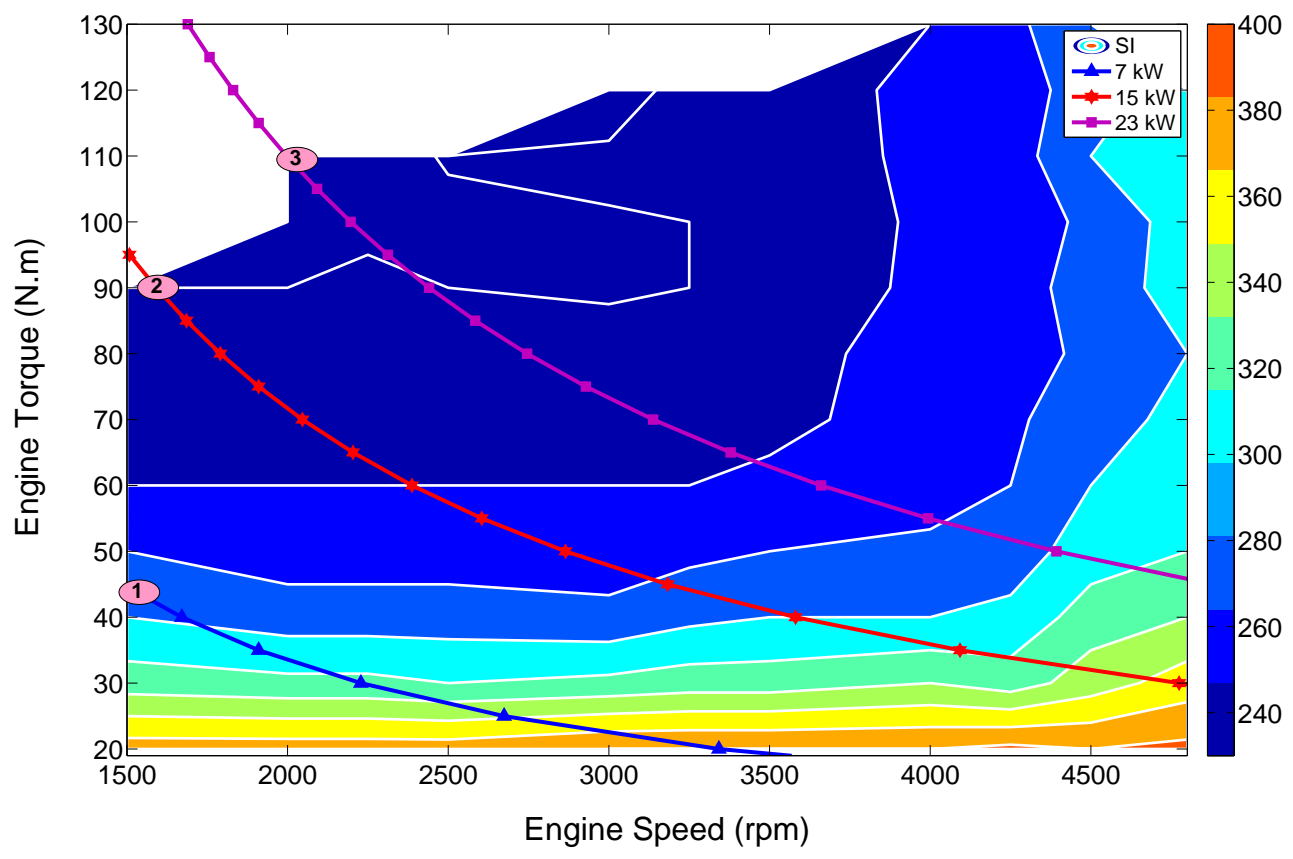

Figure 2.8: BSFC map of GM Ecotec 1.4L SI engine. The data from [7] was used to generate this Figure.

The E-motor and drive wheels speeds are synchronized with a single gear ratio that matches the E-motor maximum speed (6000 rpm) and the vehicle maximum designed velocity (100 mph). Figure 2.9] shows the E-motor speed variation in the UDDS driving cycle. The maximum E-motor speed reaches $4250 \mathrm{rpm}$, which corresponds to the vehicle velocity of $56.7 \mathrm{mph}$ in the UDDS driving cycle.

Figure 2.10 shows the E-motor efficiency map along with the E-motor operating points for the UDDS driving cycle. The E-motor efficiency in both motoring and regenerative braking modes changes in the range of $70 \%$ to $85 \%$. The E-motor works more efficiently at higher speeds and higher powers. 


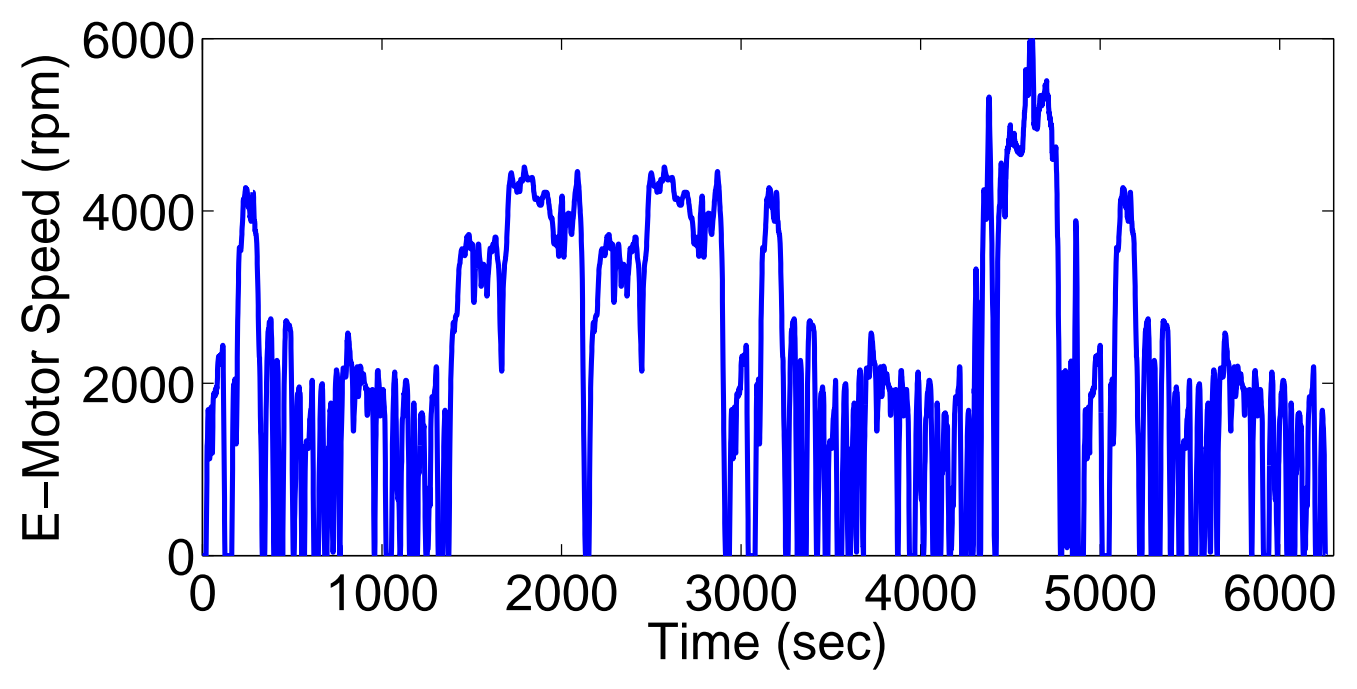

Figure 2.9: Traction E-motor speed during the UDDS driving cycle.

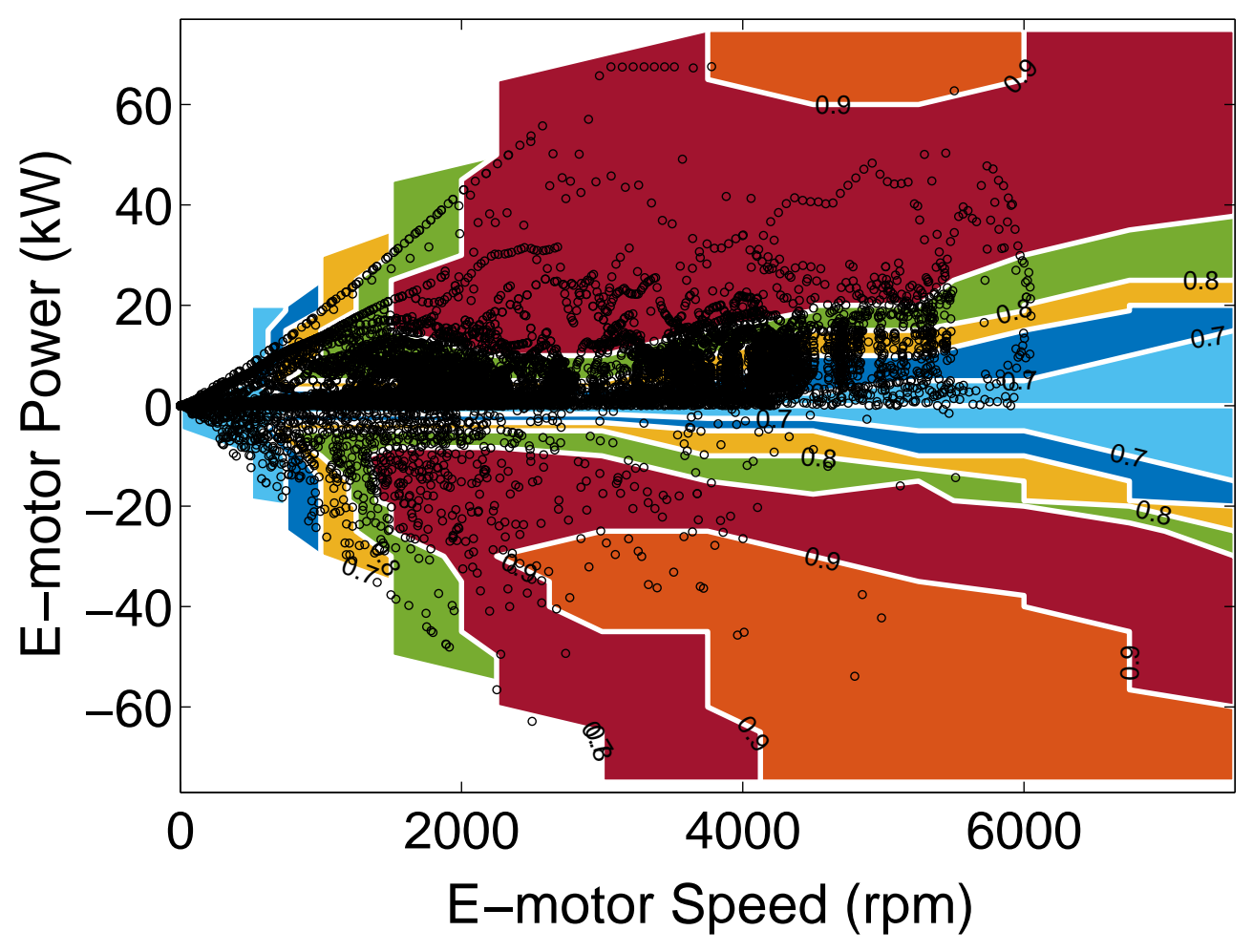

Figure 2.10: E-motor operating points, shown with ${ }^{\prime}{ }^{\prime}$, plotted over Emotor efficiency contours. 
Figure 2.11 verifies that the vehicle follows the driving cycle target speed. The vehicle speed is tracked with RMSE of $0.9 \mathrm{mph}$. The results in Figure 2.11 confirm that component sizing of the series HEV satisfies the vehicle power request in the UDDS driving cycle. Consequently, this makes the developed HEV model reliable to be used as a baseline for evaluation of the EMCs.

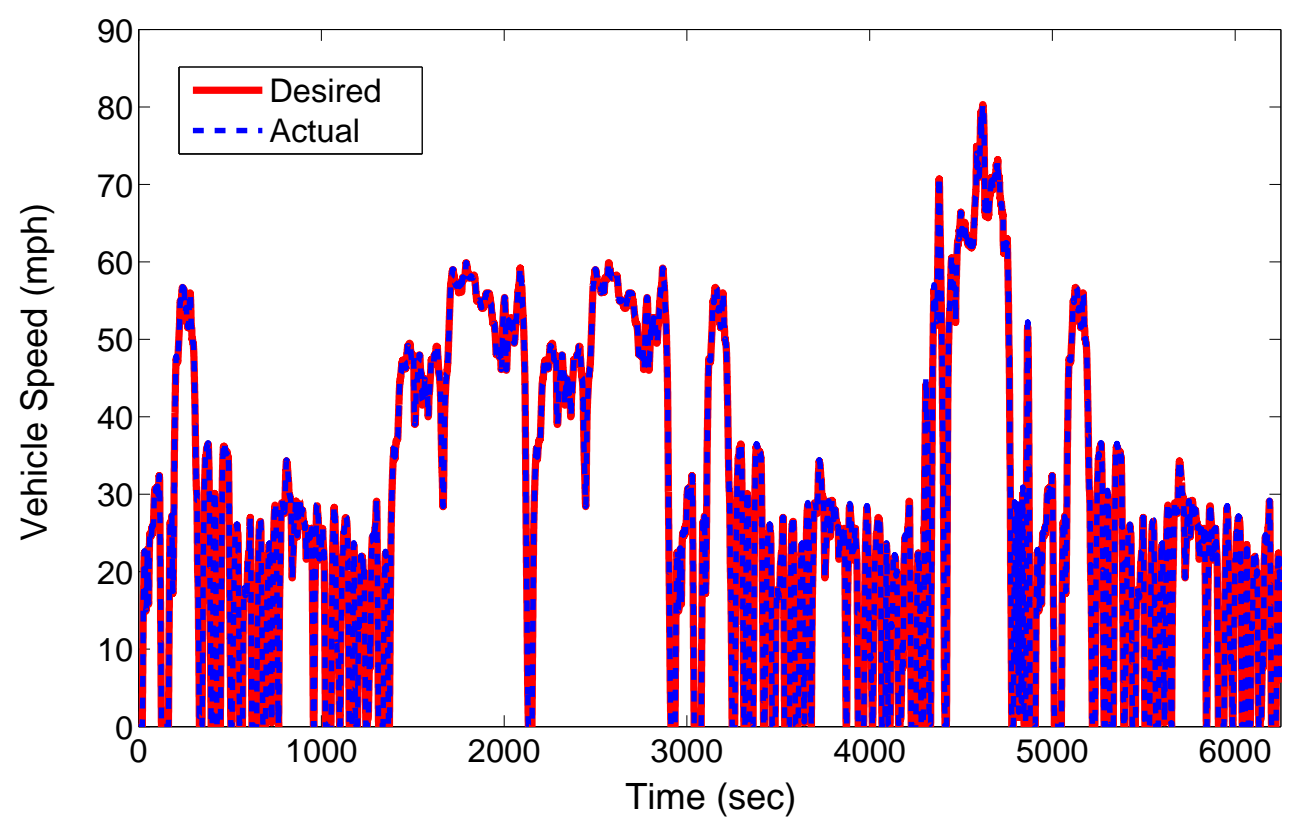

Figure 2.11: Desired vehicle speed vs. actual vehicle speed.

Figure 2.12 shows the variation of the battery SOC and engine on/off status in both thermostatic RBC and DP EMCs. In the thermostatic RBC, the SOC values specify the engine on/off status. In this chapter, for the UDDS driving cycle, when the $\mathrm{SOC}$ is lower than 0.7 , the RBC turns on the engine to charge the battery until the $\mathrm{SOC}$ reaches the starting $\mathrm{SOC}$ value (i.e., $\mathrm{SOC}=0.8$ ). In the $\mathrm{DP}$ controller, the SOC variation is significantly less than that of the thermostatic RBC. Because of the 
charge sustaining mode, the number of engine on/off transition is greater in the DP comnared to the RBC, (Figure 2.12-b).

(a)

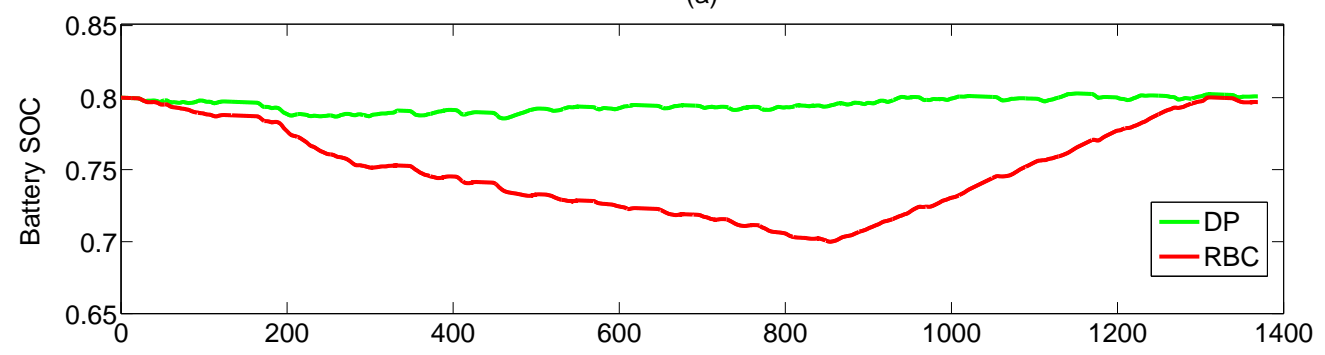

(b)

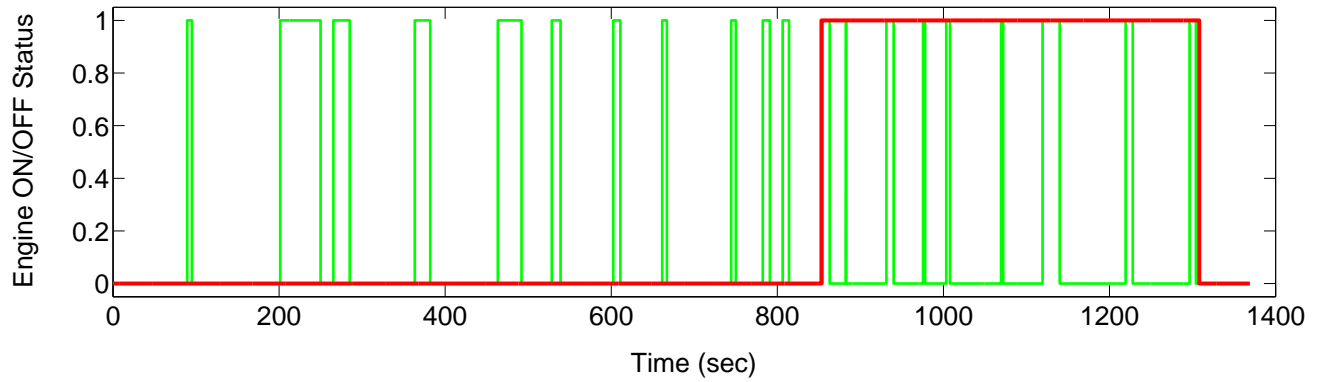

Figure 2.12: Engine on/off status and the battery SOC pattern in the DP and RBC strategies.

Figure 2.13 compares the battery output power based on the vehicle power request by using the DP control strategy. The DP strategy optimizes the engine operation based on the fuel economy in the driving cycle. In Figure 2.13, there are different modes of power distribution between the ICE and battery to meet the requested power. For example at $\mathrm{t}=95 \mathrm{sec}$, when the power request is low, the ICE simultaneously charges the battery and produces the requested power from the E-motor. Generally, the ICE can be turned on or off during braking in contrast to the RBCs that turn off the ICE during braking. The SOC variation in the DP method is small in the whole driving cycle. During the aggressive acceleration from $t=157 \mathrm{sec}$ to $\mathrm{t}=335 \mathrm{sec}$, SOC drops 
more in comparison to other times due to the large increase in the power request. In addition, in regenerative braking situations, the E-motor can recapture most of the braking power at high speeds (e.g., at $\mathrm{t}=115 \mathrm{sec}$ ), whereas at low speeds (e.g., at $\mathrm{t}=300 \mathrm{sec})$ the mechanical brake will assist the braking torque.

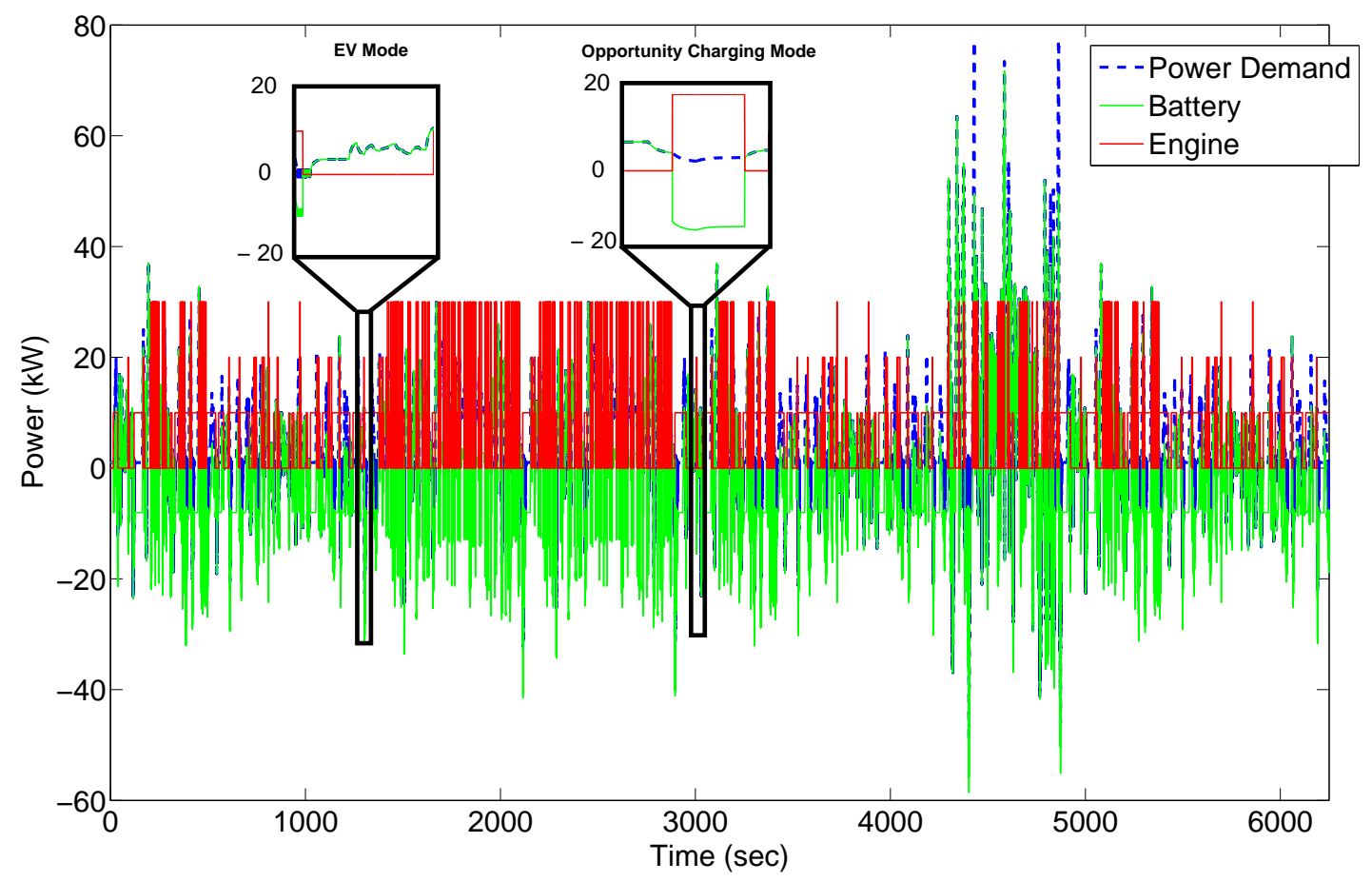

Figure 2.13: Power split profile in the DP controller.

Figure 2.14 compares the SOC variation in the DP and MPC controllers with different prediction Time Horizon (TH) values. In MPC, by increasing $\mathrm{TH}$ in the driving cycle, the SOC path will become closer to the optimal SOC path in the DP. Longer prediction TH means higher computational cost, which restricts the MPC implementation in real-time. Thus, there is a tradeoff between fuel economy improvement and 
computational load. The designed DP EMC algorithm in this chapter is computationally efficient. The simulation processing time on a $2.20 \mathrm{GHz}$ Intel processor is about 50.8 seconds for the whole combined driving cycle (i.e., $8.1 \mathrm{~ms}$ per one second of the driving cycle).

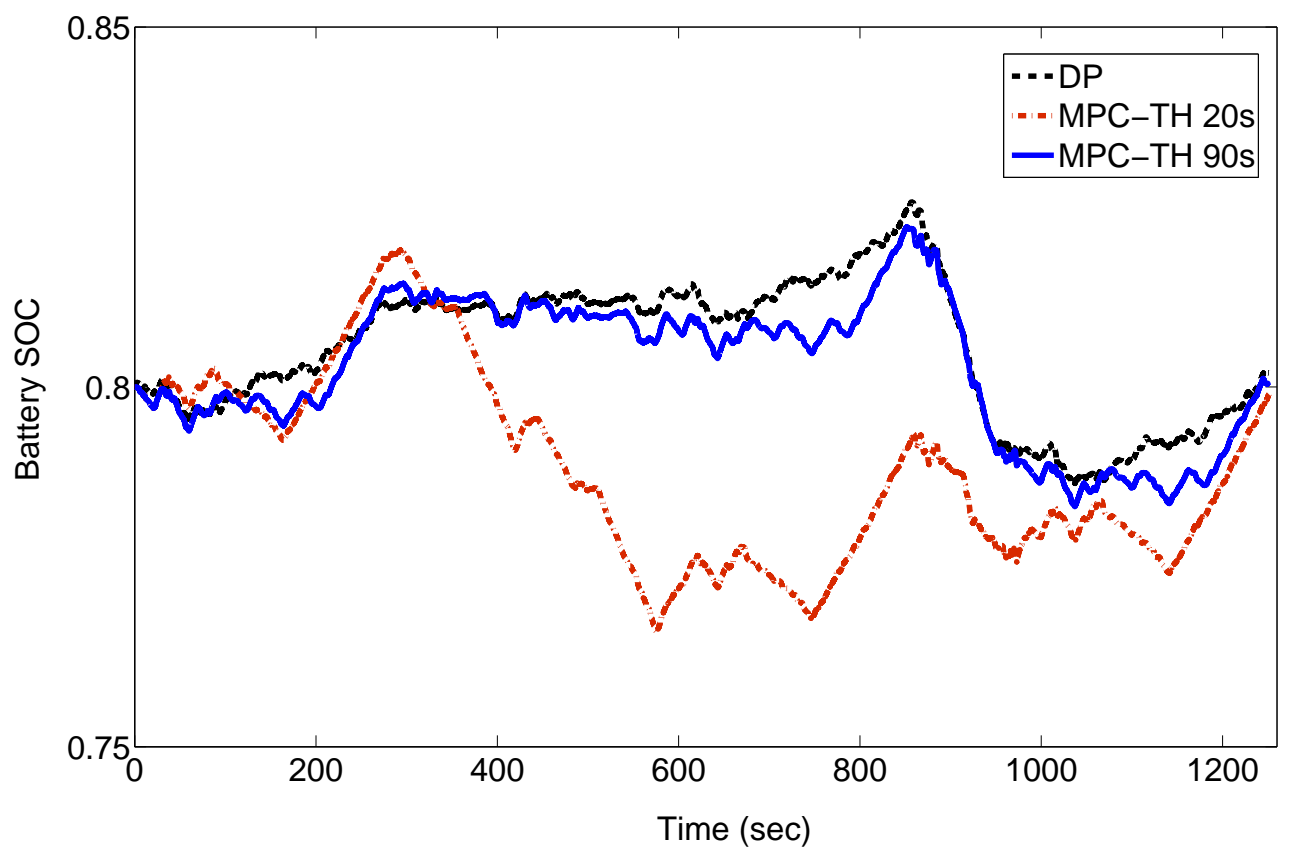

Figure 2.14: Battery SOC variation in MPC for different Time Horizons (TH) and DP controllers.

Figure 2.15 compares the fuel economy (in MPG) of the HEV with both HCCI and SI engines in series HEV. The comparison is for different EMCs including RBC, MPC, and DP. Among the EMC strategies, the DP global optimal strategy shows the best fuel economy compared to the RBC and MPC strategies. Figure 2.15 also shows the effects of $\mathrm{TH}$ length on the vehicle fuel economy. It is observed that increasing the $\mathrm{TH}$ to 70 seconds has a strong effect on improving the fuel economy. But the rate of this improvement reduces and eventually becomes negligible once the 
MPC results converge to $\mathrm{DP}$ results at $\mathrm{TH}=120$ seconds. A longer $\mathrm{TH}$ requires more computational load, but leads to more precise estimation of the future driving cycle. Therefore, obtaining high fuel economy with a low $\mathrm{TH}$ is a desirable goal for MPC EMC. $\mathrm{TH}=70$ offers a good compromise between computation load and fuel economy improvement for the HEV study in this chapter.

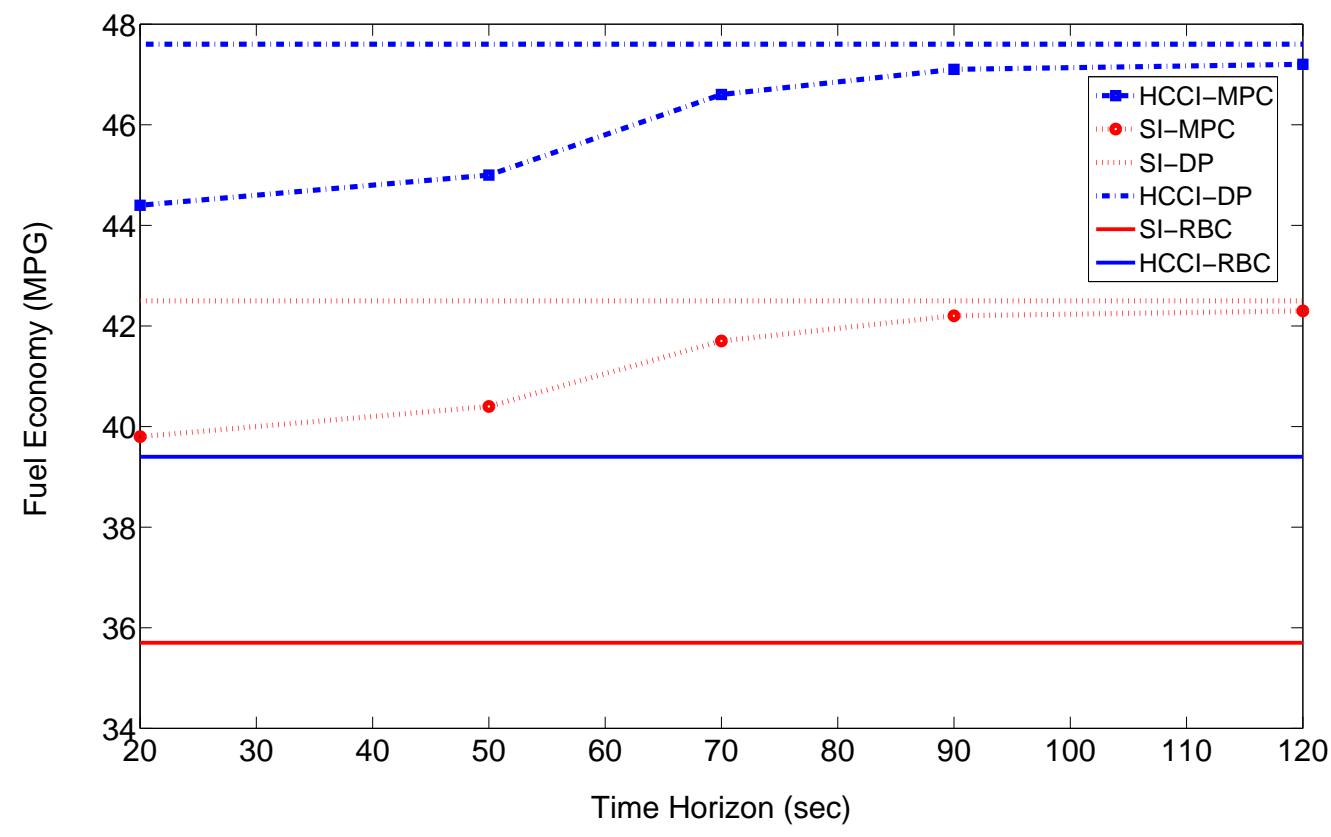

Figure 2.15: Fuel economy comparison for different EMCs including the effect of MPC Time Horizons (TH) in the series HEV for UDDS driving cycle. (EMC: 1-mode)

\subsubsection{Impact of Number of Engine Operating Points}

The impact of the number of engine operating points on the HCCI fuel economy is discussed in this subsection. To show the number of operating point impact, three 
different power levels, low, mid, and high, are selected. It should be noted that the DP formulation in this section is the same as that is in Section 2.4.2 with the only difference of the parameter " $k$ " in the Equations (2.10) to (2.14).$k \in[1,2,3,4]$, where $k=1$ represents the engine off situation, and $k=1,2$, or 3 represents the engine three selected power levels (modes). The engine power levels are selected identically for each engine to make the comparison meaningful. Within a range of 10 percent for each power level, a search is done to find the lowest BSFC value. Figure 2.3 -b and Figure 2.8 show the final three selected operating points for the HCCI and SI engines, respectively. In the single operating mode, the mid power levels (i.e., points no. 2 in Figure 2.3-b and Figure 2.8) of each engine are compared together since the engines in this operating point can produce the minimum power requirement for the vehicle. For the 2-mode operation, a low level power and a mid level power are compared (i.e., points no. 1 and 2 in the Figure 2.3-b and Figure 2.8), and for 3-mode operation all the three selected points are considered. Figure 2.16 shows the effect of number of engine operating points.

In addition, Figure 2.16 shows the effect of engine start-up fuel penalty $\left(m_{f, \text { start }}\right)$. The HCCI fuel economy improvement is constant versus the amount of $m_{f, \text { start }}$. Since the power levels for each engine are similar, the number of engine on/off switching will remain constant, which makes the HCCI fuel economy improvement independent of the $m_{f, s t a r t}$ value. Figure2.16 shows that the HCCI fuel saving increases by increasing the number of engine operating points. This can be explained by improved ICE 
efficiency when three modes are utilized in HCCI compared to that in the SI engine. Given better fuel economy results by using 3-mode EMC, the rest of the results in this chapter are presented for the 3-mode EMC.

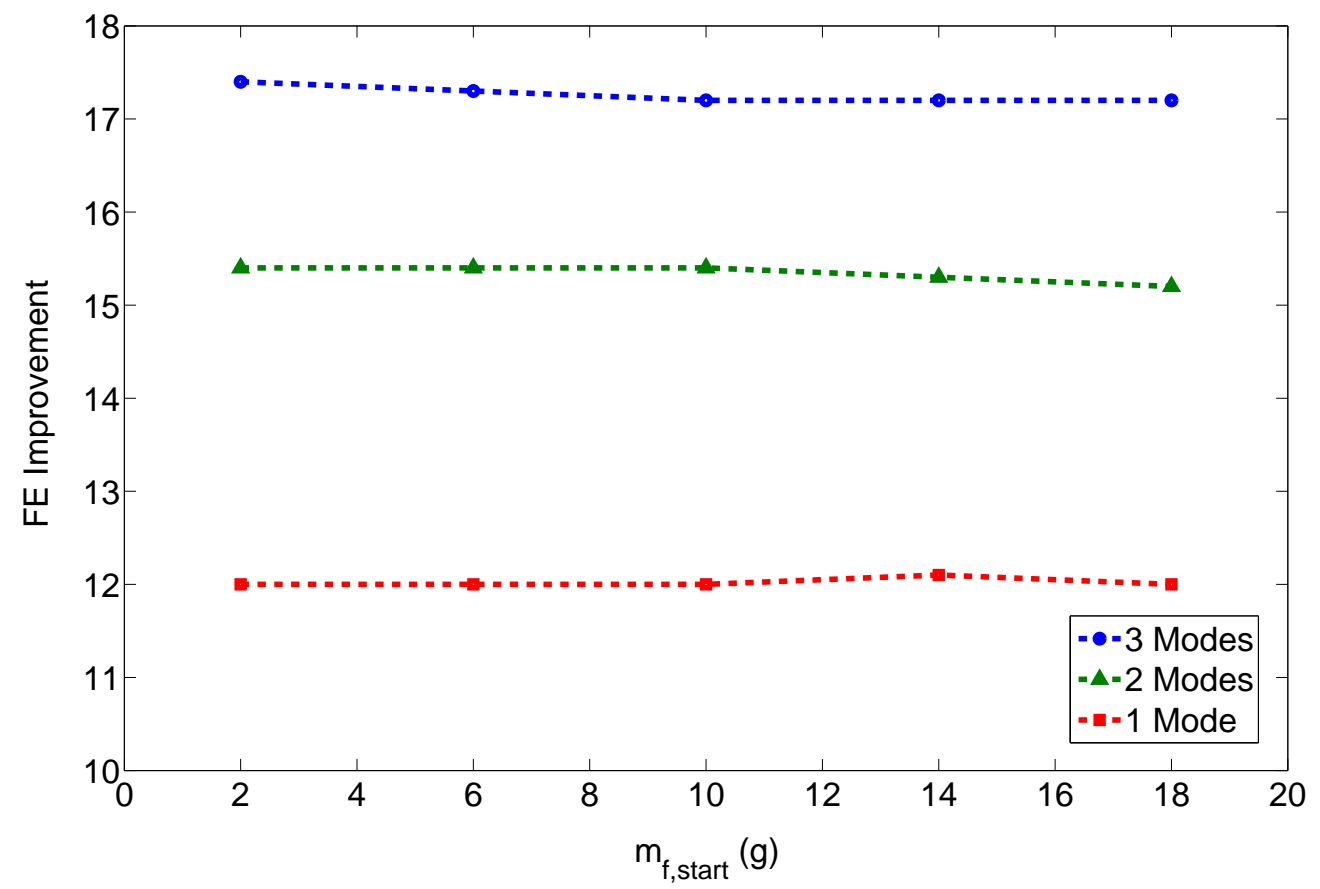

Figure 2.16: HEV fuel economy improvement by using HCCI versus SI as a function of number of engine operating points and fuel penalty for engine start-up. (EMC: DP)

Fuel economy (in MPG) of the HEV with both HCCI and SI engines in series HEV mode is shown in Table 2.5 for 3 -mode operation. The results show that using HCCI in the series HEV based on the DP EMC strategy leads to 7.7 MPG (17.7\%) fuel economy improvement for the UDDS driving cycle with three engine operating points. The MPG improvement will increase by $17.1 \%$ by using the DP strategy instead of RRB strategy for the HEV model integrated with HCCI engine. Table 2.5 also shows the effects of TH length on the vehicle fuel economy. It is observed that by increasing 
the TH from 20 to 70 seconds, the MPC strategy has a 5\% higher fuel economy for the HEV with the HCCI engine.

Table 2.5

Fuel economy comparison for different EMCs including the effect of MPC Time Horizons (TH) in series HEV. (EMC: 3-mode)

\begin{tabular}{lcc}
\hline \hline Energy Management & \multicolumn{2}{c}{ Engine Type } \\
\cline { 2 - 3 } Controller & HCCI (MPG) & SI (MPG) \\
\hline Thermostatic RBC & 43.8 & 38.6 \\
MPC -TH20s & 47.8 & 40.8 \\
MPC -TH50s & 48.5 & 41.4 \\
MPC -TH70s & 50.2 & 42.8 \\
MPC -TH90s & 50.8 & 43.3 \\
MPC -TH120s & 50.9 & 43.4 \\
DP & 51.3 & 43.6 \\
\hline \hline
\end{tabular}

\subsubsection{E-REV}

In this section, the effect of running HCCI engine in E-REV mode for the UDDS driving cycle is studied. In E-REV mode, the battery is depleted up to a lower SOC value (i.e., 0.3) and the ICE is producing power to sustain the battery SOC in the required SOC limit. Fuel economy of the HEV integrated with both HCCI and SI engines in E-REV mode are shown in Table 2.6. Comparing Tables 2.5] and 2.6 shows that the fuel economy in series is higher than E-REV. The battery output voltage decreases as the battery SOC decreases [70]. Thus, in E-REV due to the battery lower SOC range, the battery output voltage will be less than that of the series HEV. This leads to lower battery output voltage in E-REVs compared to series HEVs. Moreover, the battery requested power is constant and lower battery voltage 
means higher battery current; higher battery current drains the battery power quickly and affects the engine on/off status. Figure 2.17 shows the changes in engine on/off status in E-REV and series HEV. It can be seen that in E-REV, the number of on/off switching is greater than the series HEV.

Table 2.6

Fuel economy comparison for different EMCs including the effect of MPC

Time Horizons (TH) in E-REV mode. (EMC: 3-mode)

\begin{tabular}{lcc}
\hline \hline Energy Management & \multicolumn{2}{c}{ Engine Type } \\
\cline { 2 - 3 } \multicolumn{1}{c}{ Controller } & HCCI (MPG) & SI (MPG) \\
\hline Thermostatic RBC & 43.4 & 38.3 \\
MPC -TH20s & 47.7 & 40.6 \\
MPC -TH50s & 48.3 & 41.2 \\
MPC -TH70s & 50.3 & 42.8 \\
MPC -TH90s & 50.7 & 43.1 \\
MPC -TH120s & 50.8 & 43.2 \\
DP & 51.2 & 43.4 \\
\hline \hline
\end{tabular}

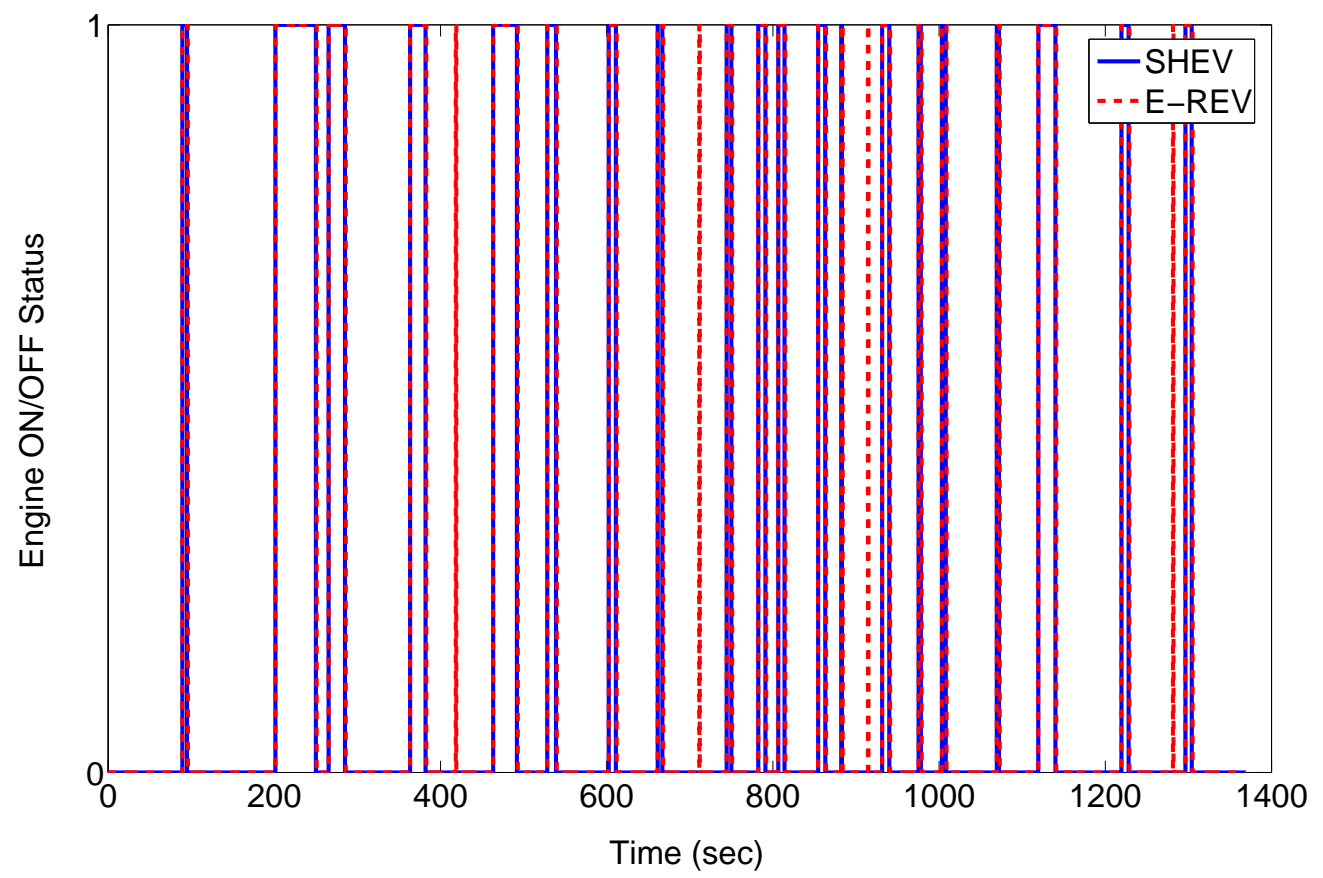

Figure 2.17: Engine on/off status in E-REV and series HEV. (EMC: 3mode DP) 
In addition, higher battery power request needs the engine to run for longer time to compensate for the driver power demand. In this case, the HCCI engine has more opportunity to work and more fuel saving is achieved compared to the SI engine; thus, the E-REV has higher fuel economy improvement versus SI engine as illustrated in Figure 2.18,

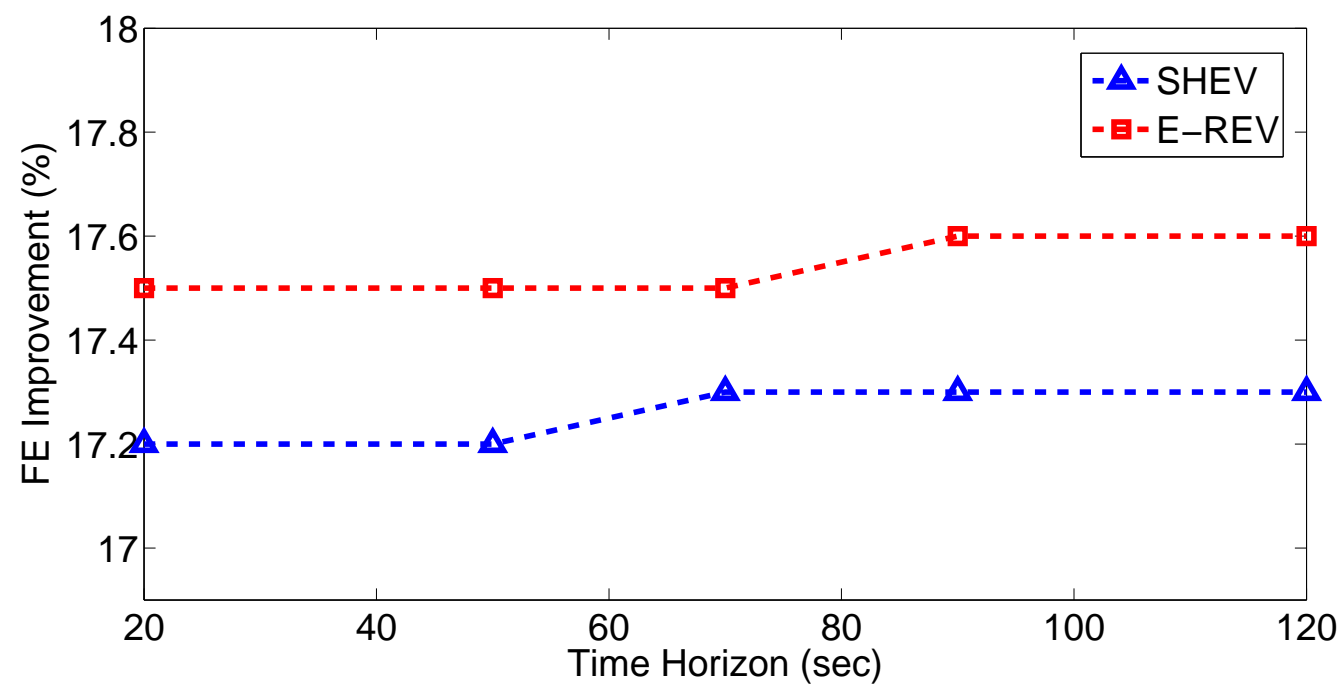

Figure 2.18: Fuel economy improvement by HCCI in different THs in series $\mathrm{HEV}$ and E-REV modes. (EMC: 3-mode)

\subsubsection{Impact of Driving Cycle}

In this subsection the impact of using various driving cycles on the HCCI fuel economy improvement for both series HEV and E-REV is explored. Three different driving cycles including UDDS, NEDC (New European Driving Cycle), and Japanese JC08 driving cycle are studied. Figure 2.19 shows the impact of the various driving cycles 
on the HCCI fuel economy improvement. The fuel economy improvement varies from $16.2 \%$ to $18.9 \%$ depending on driving cycles. Table 2.7 lists the required average power of the designed vehicle in each of the studied driving cycles. By comparing Table 2.7 and Figure 2.19, it is clear that driving cycle average power has a direct impact on the HCCI fuel economy saving over the SI engine. Thus, the fuel economy in the JC08 driving cycle is greater than that for the UDDS and NEDC driving cycles. In addition, the difference between the E-REV and series HEV fuel economy improvement is related to the difference in the length of the engine runtime as discussed in subsection 2.4.4.

Table 2.7

Average power without full regenerative braking in three driving cycles.

\begin{tabular}{lc}
\hline \hline Driving Cycles & Average Base Power (kW) \\
\hline UDDS & 4.1 \\
NEDC & 4.5 \\
JC08 & 7.5 \\
\hline \hline
\end{tabular}

\subsection{Conclusions}

In this chapter, a complete forward-in-power series HEV model integrated with HCCI and SI engine was developed in the Matlab/Simulink. All the main components, including the battery and E-motor were validated against the experimental data. The HCCI engine model was previously validated with steady-state and transient experimental data [20, 61]. Three different types of supervisory EMC strategies including $\mathrm{RBC}$, DP, and MPC were developed. The MPC strategy incorporated the DP 


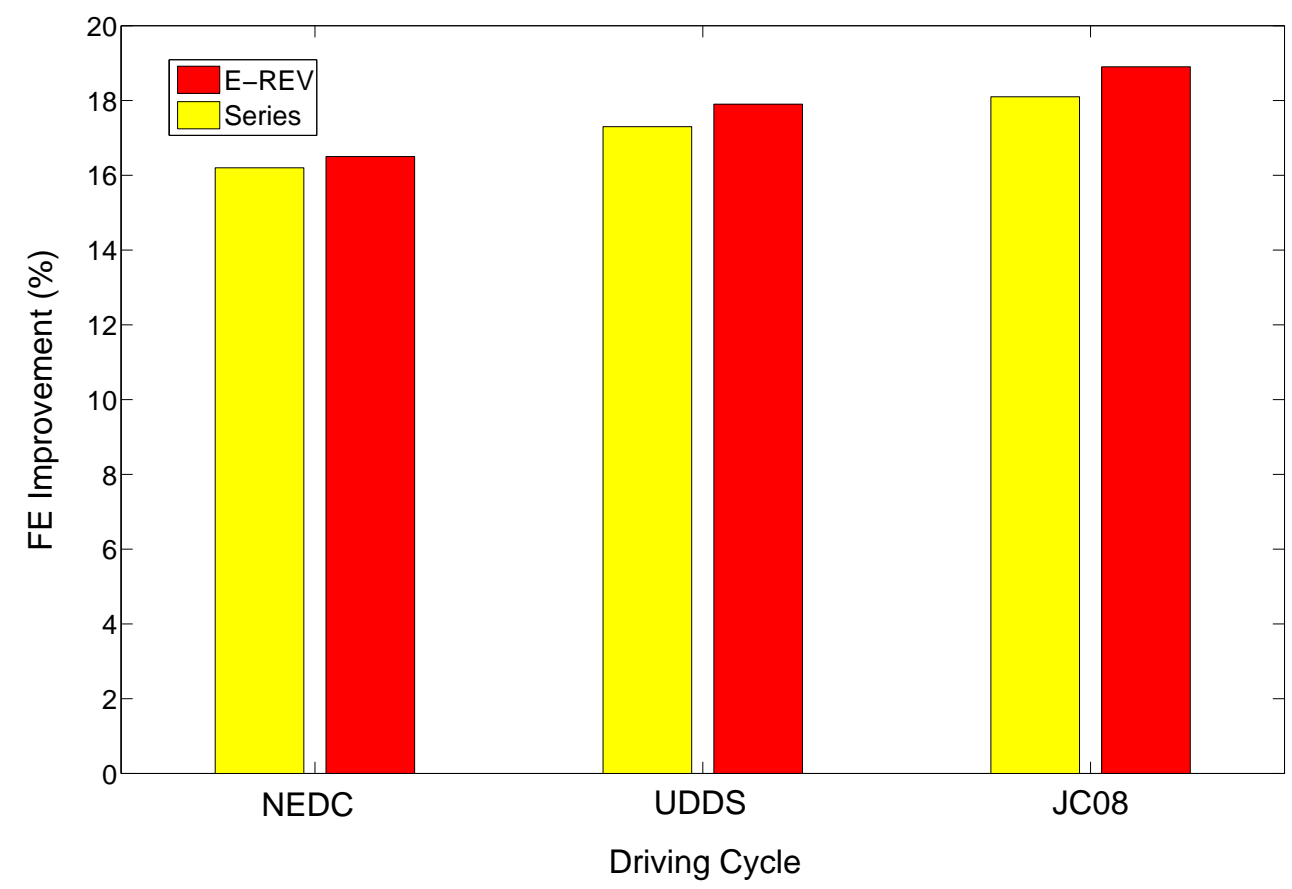

Figure 2.19: HEV fuel economy improvement by using HCCI versus SI in various driving cycles. (EMC: 3-mode DP)

method, which was applied on a moving time horizon to calculate one step ahead sub-optimal control variable. Here are the main findings from this chapter for the HEV platform studied:

$\dagger$ The results showed the HCCI engine offers significant potential for fuel saving in series HEV and E-REV architectures. In the UDDS driving cycle, integrating an HCCI engine with a series HEV powertrain can provide up to $18.9 \%$ higher fuel economy over a modern SI engine. Furthermore, the fuel economy improvement is found to be dependent on the EMC strategy applied.

$\dagger$ Increasing number of the engine operating points can increase the HCCI fuel 
economy improvement over the SI engine, though too frequent mode switches should be avoided once a high number of operating points is used. The improvement also depends on specification of each operating point.

$\dagger$ Among the three EMC strategies studied, the 3-mode DP offered the best performance with $17.1 \%$ higher fuel economy compared to the RBC for the HEV with the HCCI engine in the UDDS driving cycle.

$\dagger$ In the MPC strategy, by increasing the TH, the improvement in fuel economy increases and merges to the DP controller results. Changing the TH up to 70 seconds exhibited a strong effect on improving the fuel economy. But the rate of this improvement reduces and eventually becomes negligible once the MPC results merge to the $\mathrm{DP}$ results at $\mathrm{TH}=120$ seconds.

$\dagger$ Among all the driving cycles, those that have higher average power result in higher HCCI fuel saving. Thus, the HCCI engine operating in JC08 driving cycle offered $18.9 \%$ fuel economy improvement versus $16.2 \%$ in NEDC driving cycle in the E-REV mode.

$\dagger$ Generally, the E-REV offers a better application for the HCCI engine versus the series HEV. E-REV has $0.3 \%$ to $0.8 \%$ higher fuel economy versus the SI engine for different driving cycles. 


\section{Chapter 3}

\section{Single-Mode RCCI Engine as Range Extender 1}

Among different types of Low Temperature Combustion (LTC) regimes, Reactively Controlled Compression Ignition (RCCI) has received a lot of attention as a promising advanced combustion engine technology with high indicated thermal efficiency and low Nitrogen Oxides $\left(N O_{x}\right)$ and Particulate Matter (PM) emissions. In this study, an RCCI engine for the purpose of fuel economy investigation is incorporated in Series Hybrid Electric Vehicle (SHEV) architecture, which allows the engine to run completely in the narrow RCCI mode for common driving cycles. Three different types of Energy Management Control (EMC) strategies are designed and implemented

${ }^{1}$ This chapter has been published in Journal of Energies MDPI 2] with permission from MDPI as shown in Appendix D 
to achieve the best fuel economy. The EMC strategies encompass Rule-Based Control (RBC), offline, and online optimal controllers including Dynamic Programing (DP) and Model Predictive Control (MPC), respectively. The simulation results show $13.1 \%$ to $14.2 \%$ fuel economy saving by using an RCCI engine over a modern Spark Ignition (SI) engine in SHEV for different driving cycles. This fuel economy saving is reduced to $3 \%$ in comparison with a modern Compression Ignition (CI) engine, while $N O_{x}$ emissions are significantly lower. Simulation results show that the RCCI engine offers more fuel economy improvement in more aggressive driving cycles (e.g., US06), compared to less aggressive driving cycles (e.g., UDDS). In addition, the MPC results show that sub-optimal fuel economy is achieved by predicting the vehicle speed profile for a time horizon of $70 \mathrm{sec}$.

\subsection{Introduction}

Two thirds of the oil consumption in the world is currently used in the transportation sector and half of that goes to passenger cars and light trucks [71]. Prevalent consumption of the petroleum-based fuels leads to high greenhouse gas (GHG) emissions. The transportation sector in the U.S. and Europe accounts for around $25 \%$ of the total GHG emissions. In the U.S., the goal is to decrease the transportation GHG by $35 \%$ by 2025 [72] and in Europe the goal is to cut the transportation GHG by $67 \%$ by 2050 [73]. In this context, automakers must reduce the GHG emissions 
by introducing advanced fuel-efficient technologies and also by using alternative fuels. Low Temperature Combustion (LTC) engines include a family of Internal Combustion Engines (ICEs) technologies, including Premixed Charge Compression Ignition (PCCI), Homogeneous Charge Compression Ignition (HCCI), and Reactivity Controlled Compression Ignition (RCCI) engines, which offer low engine-out $N O_{x}$ and low soot emissions [74], and peak net indicated thermal efficiency as high as $53 \%$ [19]. Moreover, improvement in ICE fuel efficiency has the largest potential in improving Hybrid Electric Vehicle (HEV) fuel economy improvement and reducing GHG emissions, compared to enhancement in other HEV component efficiency [40]. Thus, integrating fuel-efficient LTC engines in HEVs has the potential to improve the vehicle fuel economy and decrease the GHG emissions.

However, utilizing the LTC engines in vehicles faces two major challenges: (i) limited engine operating range, and (ii) control complexity in mode transitions (e.g., SI $\leftrightarrow$ LTC, CI $\leftrightarrow$ LTC). To tackle these two challenges, this chapter investigates integration of an LTC engine with series Hybrid Electric Vehicle (HEV) by taking advantage of decoupling the ICE from the drivetrain. This allows the LTC engine to operate in a narrow operating range, removes the engine mode transients, and simplifies the LTC engine control. In addition, SHEVs are already available on the market such as the Chevrolet Volt, Fisker Karma, and BMWi3. 
Another important factor in improving fuel economy of HEV powertrains is developing optimal Energy Management Control (EMC) strategies to maximize fuel saving. Figure 3.1 lists the major EMC approaches in HEVs. These include 1) Rule-Based Controller (RBC) such as fuzzy [50] and on-off [45] strategies. These strategies are implementable in real-time applications due to their robustness and low computational cost. However, the RBC strategies do not offer the best HEV fuel saving [55, 56] due to their offline design; 2) Global optimization strategies such as Pontryagin Minimum Principle (PMP) [52] and Dynamic Programming (DP) [22, 49, 75, 76]. These strategies require the complete information of the driving cycle to determine the optimum EMC. While these global optimization strategies cannot be applied in real-time, the solution from these strategies can be used as a platform to find the ultimate fuel saving for evaluating other EMC strategies [55]; 3) Real-time optimization controllers such as Stochastic Dynamic Programming (SDP) [48, 77], Equivalent Consumption Minimization Strategy (ECMS) [47, 59, 78], and Model Predictive Controllers (MPC) [42, 58, 79]. These sub-optimal EMC strategies can be implemented in real-time. 


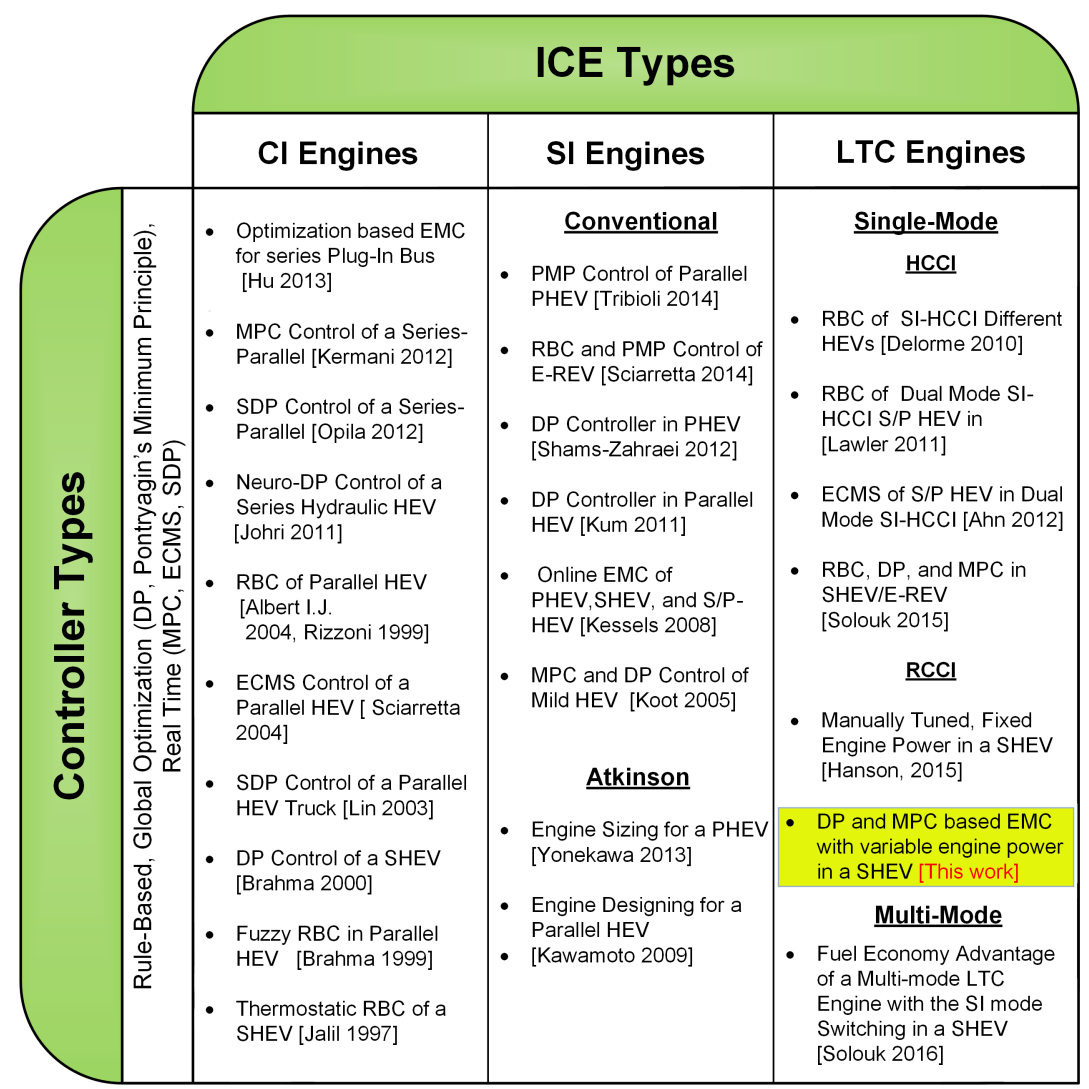

Figure 3.1: Prior studies categorized based on different types of ICEs and EMCs incorporated in HEVs.

Prior HEV studies are divided into three groups according to the ICE type (Figure 3.1). In the first group, CI engines have been used in different $\mathrm{HEV}$ architectures [42, 43, 44, 45, 46, 47, 48, 49]. The CI engines have been mostly used in Sport Utility Vehicles (SUVs), trucks, and buses [44, 46, 48, 50, 80]. In the second group, SI engines have been used in HEV [51, 52, 53]. Recently, SI engines with Atkinson cycle have become more popular in the market (e.g., Ford C-Max, Honda Accord PHEV, Lexus RX 450h, and Toyota Prius). In [28], an Atkinson cycle SI engine was used by Toyota R\&D group to increase the fuel economy benefit of HEVs. In another study [27], a 
Honda Accord PHEV was redesigned based on the Atkinson cycle and $10 \%$ lower fuel consumption was reported compared to the SI engine.

Few studies are found in the literature that investigated integrating LTC engines with HEVs. Among different types of LTC engines, HCCI was the first type explored in hybrid electric powertrains. In the first study at Argonne National Laboratory in U.S., the effects of using a dual mode SI-HCCI engine in different vehicle electrification levels were analyzed [35]. Their simulation results predicted $6 \%$ to $15 \%$ fuel consumption reduction, depending on powertrain configurations and driving cycles. In another study [34], fuel economy improvement of an HCCI engine versus an SI engine for both mild and medium parallel HEVs was investigated. The authors reported a range from $17 \%$ to $35 \%$ fuel economy improvement with using a dual-mode SI-HCCI in comparison to a conventional SI non-hybrid powertrain. In both studies [34, 35], a dual mode (SI-HCCI) engine was used with a rule-based EMC. Another study [59] was conducted to investigate the effect of utilizing a dual mode SI-HCCI engine on a power-split HEV acceleration performance. The authors quantified the trade-off between the vehicle fuel economy and the vehicle performance.

RCCI is the second type of LTC engine that has been recently investigated for HEVs. The study in [37] is the only study available in the literature for an RCCI-based HEV. In [37], researchers at the University of Wisconsin-Madison and Oak Ridge 
National Laboratory in U.S. used an RCCI engine in a series hybrid electric powertrain. The authors tested the vehicle for the US Environmental Protection Agency (EPA) Highway Fuel Economy Test (HWFET) procedure to measure the vehicle fuel economy and emissions. Based on simulation results, the authors predicted that a series-parallel RCCI-HEV configuration will lead to $12 \%$ fuel economy improvement for the Chevrolet Volt, which currently uses a SI engine. The study in [37] included preliminary results and no model-based EMC strategy was used for optimizing the energy balance between the battery and ICE energy sources. In addition, the results were presented only for the HWFET driving cycle. The EMC type and driving cycles will affect the RCCI-HEV performance and fuel saving results, as will be shown in this chapter.

To the best of the author's knowledge, this is the first study undertaken to investigate the fuel economy benefit of integrating an RCCI engine in SHEV with advanced EMCs. The contribution from this chapter is threefold. First, it investigates the ultimate fuel saving of a dedicated RCCI-mode engine in SHEV configuration. Second, it investigates the effect of EMCs on the potential of fuel economy improvement for SHEV using an RCCI engine. Third, it studies the impact of driving cycle and also the battery initial SOC on the fuel economy of the RCCI-based SHEV.

This chapter is organized as follows. In Section [3.2, the developed HEV model is presented and the main components of the HEV model are described. Section 3.4 
explains three different EMC strategies including on-off RBC, global optimization DP, and MPC. In Section 3.3 the optimum number of engine operating points are selected with regards to the fuel economy. The SHEV results are presented in Section 3.5. Finally, Section 3.6 summarizes all findings from this chapter.

\subsection{SHEV Model Description}

A SHEV with specification listed in Table 3.1 is modeled in this section.

Table 3.1

Vehicle specifications.

\begin{tabular}{lc}
\hline \hline Parameters & Values \\
\hline $\mathrm{M}(\mathrm{kg})$ & 1431 \\
$f_{r}(-)$ & 0.01 \\
$\mu(-)$ & 0.8 \\
$\rho\left(\mathrm{kg} / \mathrm{m}^{3}\right)$ & 1.224 \\
$C_{d}(-)$ & 3.25 \\
$R_{w}(\mathrm{~m})$ & 0.381 \\
$A\left(\mathrm{~m}^{2}\right)$ & 2.0 \\
$G r(-)$ & 3.25 \\
$P_{b}(\mathrm{~kW})$ & 215 \\
Battery capacity (kWh) & 18.5 \\
E-motor peak power (kW) & 75 \\
E-motor peak torque (N.m) & 240 \\
E-motor maximum speed (rpm) & 7500 \\
E-motor maximum efficiency (-) & 0.94 \\
Generator efficiency (-) & 0.95 \\
\hline \hline
\end{tabular}

The SHEV model encompasses different submodels including the RCCI engine, Longitudinal Vehicle Dynamics (LVD), E-motor, and battery. The model is a forwardin-power HEV model developed in Matlab $^{\circledR} /$ Simulink to evaluate the EMC strategies 
and fuel economy benefits. Using the LVD model, the vehicle speed profile is calculated based on the available supplied traction torque by the E-motor after subtracting for drag and rolling resistance forces. The supervisory controller specifies the battery required power and the engine power based on the battery State Of Charge (SOC), and the driver power demand. Figure 3.2 shows the high level schematic of the model. The description for sizing and selection of HEV electrical components are found in [1]. The SHEV model is briefly explained as follows.

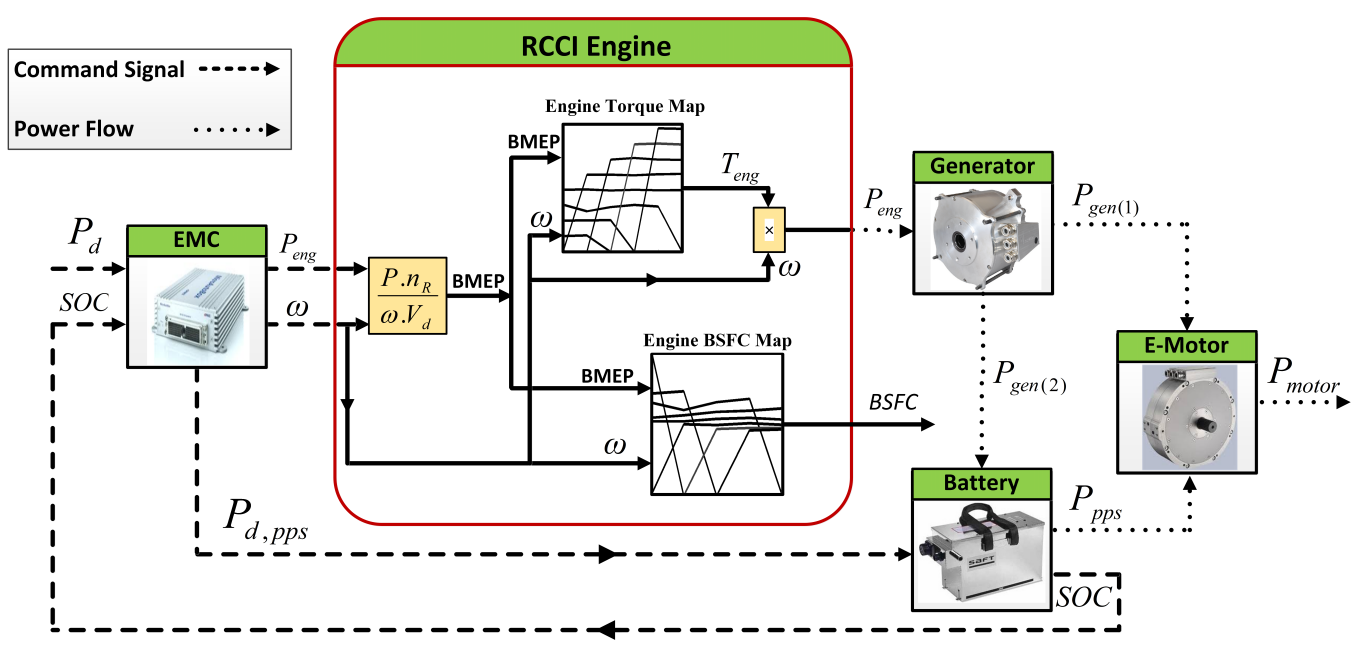

Figure 3.2: A schematic diagram to illustrate the power and control flows among the main components of an RCCI-based SHEV.

\subsubsection{IC Engines}

Three different ICEs will be studied as part of the SHEV. These ICEs include RCCI, CI (diesel), and SI (gasoline) engines. The RCCI engine used in this chapter is based on a modified GM Z19DTH diesel engine. The diesel engine was converted to an RCCI engine by a group of scholars from the University Wisconsin-Madison and Oak 
Ridge National Laboratory [8]. The RCCI engine has a new piston design and its compression ratio is reduced from 17.1 to 15.1 [8]. Table 3.2 lists the RCCI engine specifications and Figure 3.3 shows the RCCI engine Brake Specific Fuel Consumption (BSFC) map using the data from [8].

Table 3.2

Specification of GM Z19DTH diesel engine converted for RCCI operation [8].

\begin{tabular}{lc}
\hline \hline Parameters & Values \\
\hline Bore (mm) & 82 \\
Stroke (mm) & 90.4 \\
Displacement (L) & 1.9 \\
Compression Ratio & 15.1 \\
Max. Power (kW) @ 3000 rpm & 32.0 \\
Engine Speed Range (rpm) & $1000-3500$ \\
\hline \hline
\end{tabular}

The engine speed range is from $1000 \mathrm{rpm}$ to $3500 \mathrm{rpm}$. The BSFC map of the engine (Figure 3.3) was created using the engine Brake Thermal Efficiency (BTE) data from [8] and Lower Heating Value (LHV) of fuels. The RCCI engine model is designed such that it takes the required Brake Mean Effective Pressure (BMEP) and the required engine power and then calculates the engine fuel consumption. 


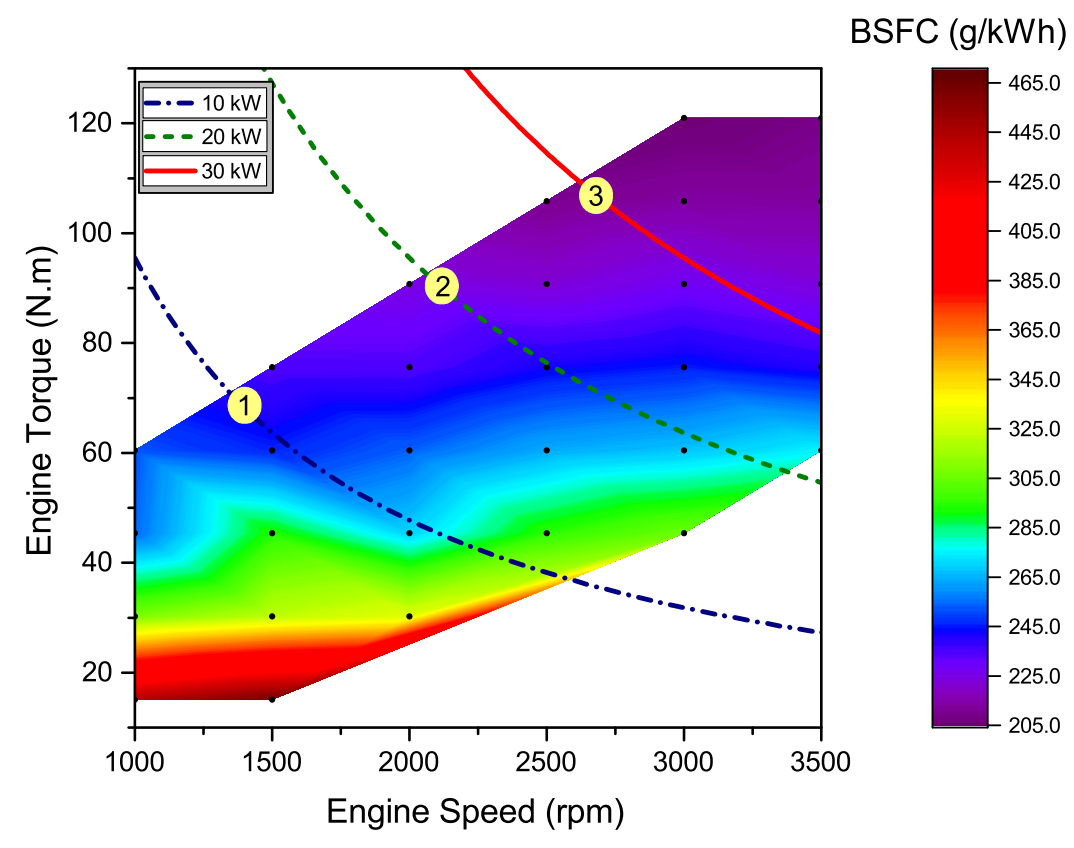

Figure 3.3: BSFC map $(\mathrm{g} / \mathrm{kWh})$ of the RCCI engine in this chapter. The data from [8] was used to generate this figure.

For the comparative study, the BSFC model of a CI engine and a SI engine are included in the HEV model. The same base engine is used for both RCCI and CI (diesel) modes. Thus, the original GM Z19DTH diesel engine data from [8] is used for the CI engine model. Figure 3.4 shows the BSFC map for the CI engine. For a fair comparison, the SI engine is selected such that it has optimum power rating for the HEV size in this chapter. To this end, a GM A14XFL SI (gasoline) engine from a mid-size HEV on the market is chosen. 


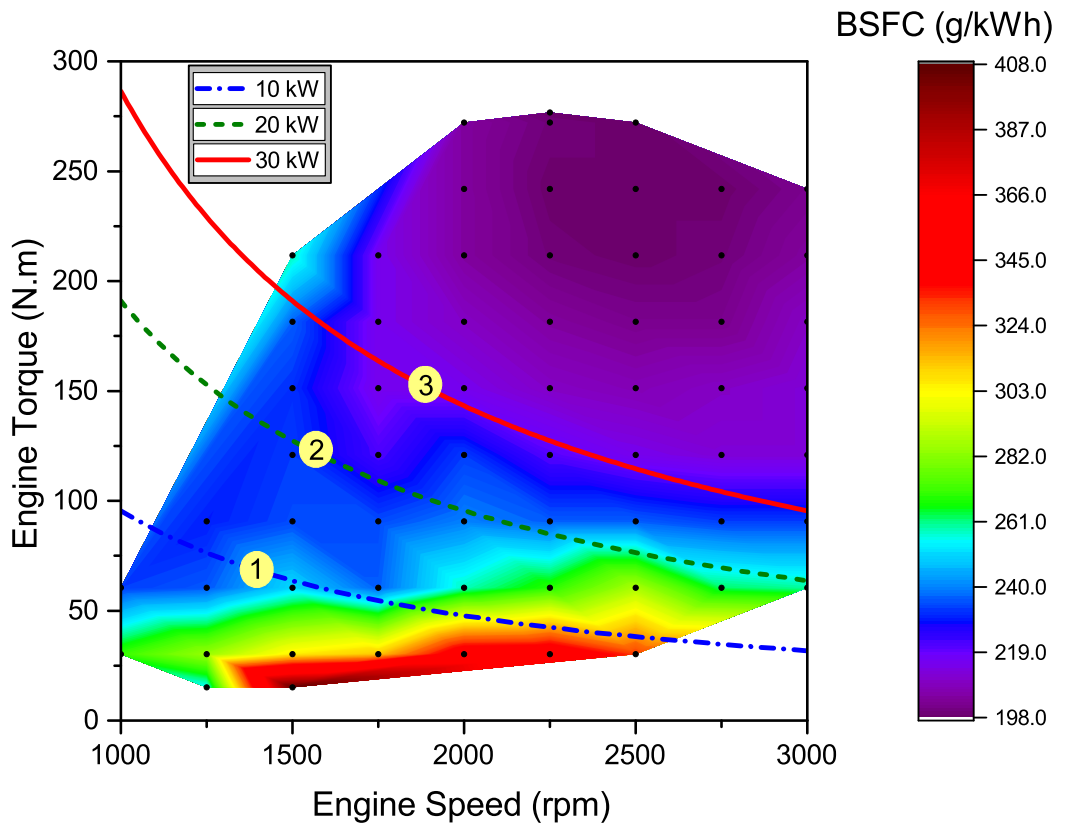

Figure 3.4: $\quad$ BSFC map ( $\mathrm{g} / \mathrm{kWh}$ ) of the GM Z19DTH CI (diesel) engine. The data from [8] was used to generate this figure.

Figure 3.5 shows the SI engine's BSFC map based on the data from [7]. This figure demonstrates that the selected engine operating points (i.e., points \# 1, 2, 3) are located in the lowest BSFC region of the SI engine. 


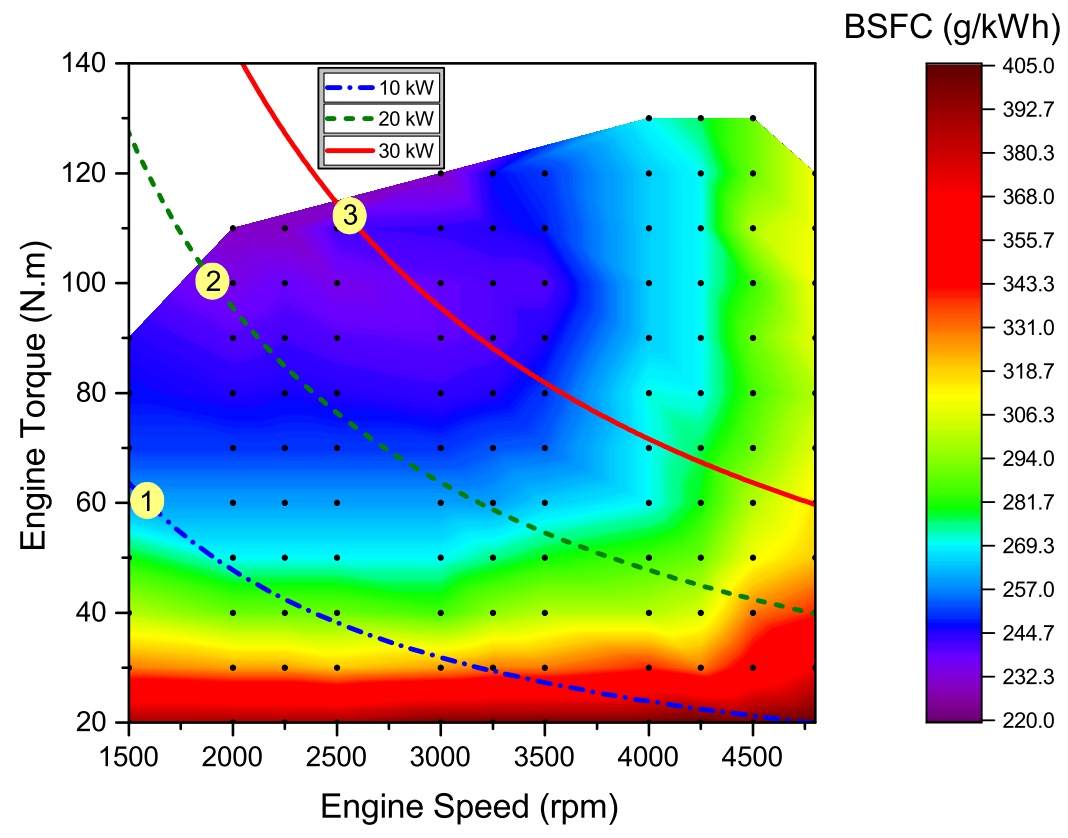

Figure 3.5: $\quad$ BSFC map (g/kWh) of GM A14XFL SI (gasoline) engine. The data from [7] was used to generate this figure.

\subsubsection{Longitudinal Vehicle Dynamics}

The LVD model calculates the vehicle actual speed $V$ based on the vehicle traction and resistance forces and the dynamics associated with the vehicle. The following equation shows the governing dynamic model used for the LVD model:

$$
M \frac{d V}{d t}=F_{\text {traction }}-F_{\text {drag }}-F_{\text {roll }}-F_{\text {gravity }}
$$


where $M$ is the vehicle total mass, $F_{\text {gravity }}, F_{\text {drag }}$, and $F_{\text {roll }}$ are gravity force, aerodynamic drag force, and rolling resistance force, respectively. The resistive forces are calculated from the following equations:

$$
\begin{gathered}
F_{\text {gravity }}=M g \sin \theta \\
F_{\text {drag }}=\frac{1}{2} \rho A C_{d} V^{2} \\
F_{\text {roll }}=M g f_{r}\left(1+\frac{V}{100}\right)
\end{gathered}
$$

where $\theta$ is the road slope, $C_{d}$ is the vehicle drag coefficient, $\rho$ is the air density, $A$ is the vehicle frontal area, and $f_{r}$ is the rolling resistance coefficient. The main parameters of the LVD model are listed in Table 3.1.

\subsubsection{E-motor Model}

A single gear ratio $75 \mathrm{~kW}$ E-motor [6] is utilized in this chapter. The E-motor requested power is calculated based on the supervisory controller's demanded power as the input to a map-based E-motor model. The E-motor model includes an efficiency map versus power request and rotational speed up to the maximum of 7500 rpm. Based on the E-motor operating point, the E-motor actual generated power is obtained from the efficiency map. Then, the E-motor traction torque is calculated 
using the electrical available power and the motor speed. The available electrical power consists of the E-motor generated power and the battery power by including a constant auxiliary load used for the entire driving cycle [81]. The E-motor efficiency map covers both traction $\left(\eta_{\text {traction }}\right)$ and regenerative braking $\left(\eta_{\text {braking }}\right)$ modes. Equation (3.5) shows the torque relation of the E-motor:

$$
T_{\text {motor }}= \begin{cases}\frac{P_{\text {available }} \eta_{\text {traction }}}{\omega_{\text {motor }}} & \text { (a) traction } \\ \frac{P_{\text {available }}}{\omega_{\text {motor }} \eta_{\text {braking }}} & \text { (b) regenerative braking }\end{cases}
$$

where, $P_{\text {available }}$ refers to the available electrical power in the battery. Consequently, the vehicle traction force $\left(F_{\text {traction }}\right)$ and the E-motor speed $\left(\omega_{\text {motor }}\right)$ are calculated by:

$$
\begin{aligned}
F_{\text {traction }} & =\frac{T_{\text {motor }}}{R_{w}} G r \\
\omega_{\text {motor }} & =\frac{V}{R_{w}} G r
\end{aligned}
$$

where, $R_{w}$ is radius of the wheels and $G r$ is the final gear ratio between the E-motor and the driveline. The $G r$ is designed to match the vehicle maximum designed speed with the E-motor maximum speed (i.e., the maximum vehicle speed is $100 \mathrm{mph}$ and the maximum E-motor speed is $7500 \mathrm{rpm}$ ). The actual vehicle speed is obtained from the LVD model. In addition, the E-motor was sized and selected based on the required traction torque and power for the vehicle gradeability and acceleration performances 
[1]. Table 3.1 lists the main specifications of the E-motor.

\subsubsection{Battery Model}

Based on the E-motor operating point, the E-motor actual generated power is obtained from the efficiency map. The supervisory controller specifies the battery required power, as shown in Figure 3.2. A battery energy capacity and power is sized to meet the SHEV design requirements. The battery supplies 40 miles AER, which is a common range for SHEVs such as 2015 Cadillac ELR. A battery model is employed to estimate the battery SOC and the battery voltage. The battery model is from [63] and it is widely used in previous HEV and EV investigations. This model extracts a nonlinear model (experimental data [5]) for the battery characteristic discharge curve. Finally, the $S O C$ is calculated by:

$$
S O C=1-\frac{\int i d t}{Q}
$$

where $i$ is the battery current and $Q$ is the battery nominal capacity. More discussions on the battery sizing and models are available in [1]. 


\subsubsection{Vehicle Acceleration and Braking Performance}

The HEV model is implemented in Matlab ${ }^{\circledR} /$ Simulink. The vehicle acceleration performance was designed to reach $60 \mathrm{mph}$ in 12.2 seconds, which is similar to a common mid-size HEV on the market [65]. The vehicle braking performance was designed such that to be able to stop from $60 \mathrm{mph}$ in less than 53.4 meters similar to that in [65].

\subsubsection{Model Validation}

The BSFC maps of the RCCI and CI engines are developed experimentally in [8] and the experimental data are used in this chapter. The models for two other HEV major components including the E-motor and battery were validated against experimental data [1]. The selected E-motor is UQM PowerPhase 75 and the E-motor efficiency model was validated against the manufacturer's experimental data [6] with RMSE less

than $1.5 \%[1]$. Moreover, the battery model was validated against the experimental data from SAFT VL7P Li-ion battery [5] with the RMSE of $0.17 \mathrm{~V}$ [1]. 


\subsection{Selection of Engine Operating Points}

In SHEVs, the engine can operate independent of the vehicle speed and the wheel torque. Thus, the engine has the opportunity to work most of the time at high efficiency operating points and low engine-out emission region. In this section, the engine operating points are selected for three different constant power levels as low, mid, and high to fulfill the vehicle power requirements and also to guarantee low engine BSFC and low engine-out emissions. The low BSFC and low engine-out emission constraints are discussed in subsections A and B.

A. Engine BSFC Constraint: The selected operating points are at three power levels designated as $10 \mathrm{~kW}, 20 \mathrm{~kW}$, and $30 \mathrm{~kW}$. Within a range of 10 percent for each power level, a search is done to find the lowest BSFC value. Figure 3.3 shows the three final selected operating points for the RCCI engine. In the single operating mode, the mid power level (i.e., point no. 2 in Figure 3.3) is selected since the engine can provide the mean power requirement for the vehicle at this operating point. For the 2-mode operation, a low level power and a mid level power are selected for the engine operating points (i.e., points no. 1 and 2 in Figure 3.3), and for the 3-mode operation all the three selected points are considered.

B. Engine Emissions Constraint: The engine $\mathrm{HC}, \mathrm{CO}$, and $N O_{x}$ emissions are considered in selecting the engine operating points. $\mathrm{CO}$ and $\mathrm{HC}$ conversions in Diesel 
Oxidation Catalysts (DOC) are a function of the exhaust gas temperature. Thus, the engine operating points are selected to meet the minimum DOC light-off temperature. The RCCI engine exhaust gas temperature for different engine speeds and torques are shown in Figure [3.6 based on the data from [8] along with the selected operating points. It is shown in Figure 3.6 that the exhaust gas temperature for all of the three engine operating points are above $290{ }^{\circ} \mathrm{C}$. Moreover, in reference [82] it is shown that the DOC for the same RCCI engine achieved $90 \%$ and $100 \% \mathrm{HC}$ and CO conversions, respectively, when the exhaust gas temperature in the RCCI engine was higher than $290{ }^{\circ} \mathrm{C}$. Thus, the selected RCCI engine operating points meet the DOC light-off temperature to achieve low $\mathrm{HC}$ and $\mathrm{CO}$ emissions, similar to those in the CI and SI engines. 


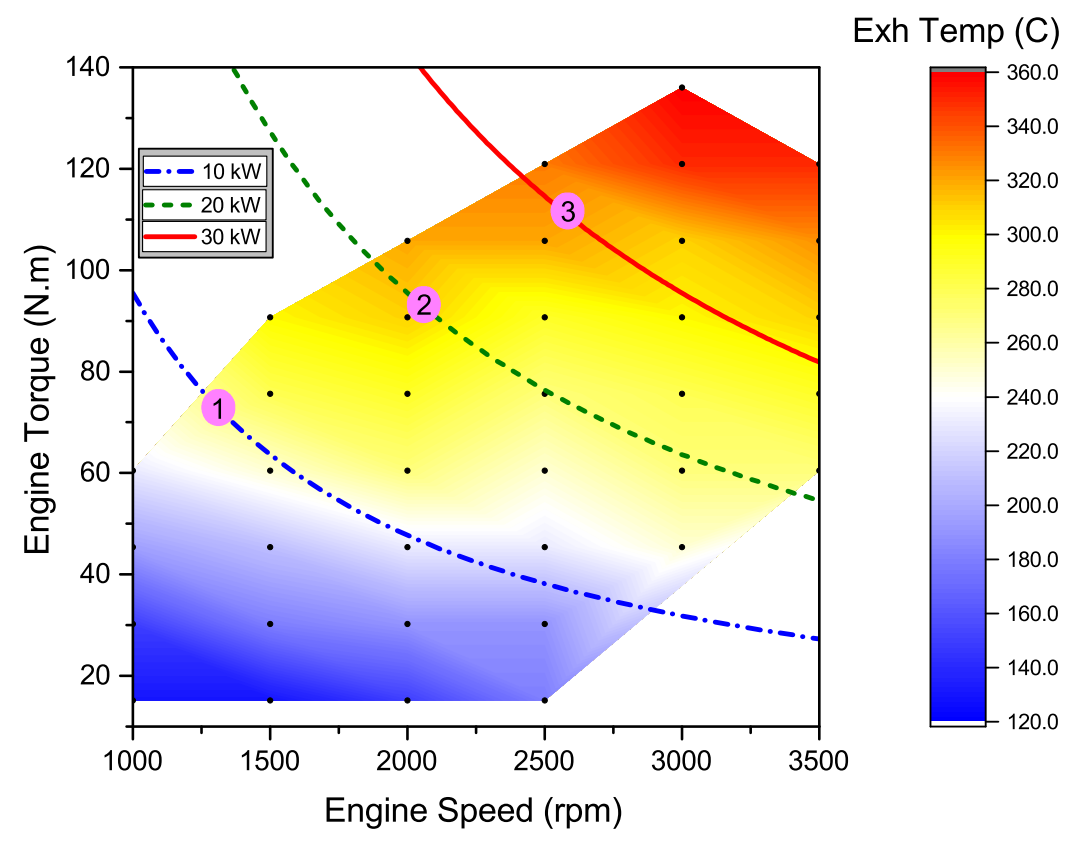

Figure 3.6: RCCI exhaust gas temperature map for selecting the RCCI engine operating points. The data from [8] was used to generate this figure.

In addition to the $\mathrm{HC}$ and $\mathrm{CO}$ emissions, the $N O_{x}$ emission is also taken into account for selecting the engine operating points. Table 3.3 lists the $N O_{x}$ emission associated with the selected operating points in both RCCI and CI engines. The table shows that the selected engine operating points in RCCI produces much less $N O_{x}$ compared to the selected operating points in the CI engine. This is a well-recognized fact in literature [8, 19, 83] and considered as one of the main advantages of RCCI engines. Thus, a smaller $N O_{x}$ aftertreatment system is required in RCCI engines, compared to CI engines. 
Table 3.3

Engine-out $N O_{x}$ emissions in RCCI and CI engines operating points. The data is from [8].

\begin{tabular}{ccc}
\hline \hline Engine Operating Points & \multicolumn{2}{c}{ NOx Emission (g/kW-hr) } \\
\cline { 2 - 3 } & RCCI & CI \\
\hline Point 1 & 0.4 & 0.6 \\
Point 2 & 0.4 & 6.0 \\
Point 3 & 0.4 & 6.0 \\
\hline \hline
\end{tabular}

Given the SI and CI engines are production engines, the engines and exhaust aftertreatment systems are optimized to meet $\mathrm{HC}, \mathrm{CO}$, and $N O_{x}$ constraint for the emission standard. In addition, the discussions above demonstrate that the RCCI engine produces low $\mathrm{HC}, \mathrm{CO}$, and $N O_{x}$ emissions in the three selected operating points. Thus, all the three engine operating points in the SI, CI, and RCCI engines have both low BSFC and low emissions advantage over the other engine operating regions at the designated power levels.

In this section, the effect of the number of the engine operating points on the HEV fuel economy is also discussed. Table 3.4 shows the effect of number of engine operating points and the engine start-up fuel penalty on the RCCI-SHEV fuel economy.

Table 3.4

RCCI engine fuel economy values in the SHEV architecture as a function of engine start-up fuel penalty and number of operating modes utilized in the engine. (EMC: DP)

\begin{tabular}{cccc}
\hline \hline Fuel Penalty & \multicolumn{3}{c}{ Engine Modes } \\
\cline { 2 - 4 }$m_{f, \text { start }}(\mathrm{g})$ & 1-Mode $(\mathrm{MPG})$ & 2-Modes $(\mathrm{MPG})$ & 3-Modes $(\mathrm{MPG})$ \\
\hline 2 & 47.8 & 48.0 & 48.8 \\
6 & 47.3 & 47.5 & 48.1 \\
10 & 46.9 & 46.9 & 47.6 \\
14 & 46.3 & 46.3 & 47.0 \\
\hline \hline
\end{tabular}


The simulation results in Table 3.4 show that the RCCI fuel economy increases by increasing the number of engine operating points. This can be explained by improved overall ICE efficiency when three modes are utilized in RCCI for different driver power request levels. Given better fuel economy results by using the 3-mode EMC, the rest of the results in this chapter are presented for the 3-mode EMC.

\subsection{Energy Management Controller (EMC) De- sign}

The EMC strategies in this chapter aim to $(i)$ keep the hybrid powertrain to operate in the Charge Sustaining (CS) mode, (ii) fulfill the driver power demand, and (iii)

maximize the powertrain fuel saving [84]. Here, three types of EMC strategies are designed for the SHEV-RCCI powertrain. These strategies include on-off RBC, DP, and MPC. Desired SOC window of $0.55 \leqslant S O C \leqslant 0.9$ is used in all of the three EMCs.

\subsubsection{EMC Type I: RBC}

In on-off RBC, the EMC rules are designed heuristically without driving cycle information. The battery SOC is the only input to the control unit, which forces the 
battery to keep the SOC in the desired window (i.e., $0.55 \leqslant S O C \leqslant 0.9$ ), by controlling the engine on/off status. The on-off RBCs cannot adapt their rules with changing driving cycles. This results in non-optimal efficiency for a wide operating range. Simple implementation for real-time EMC applications is the major advantage of on-off RBC strategies [66]. Here, a heuristic RBC is designed to keep the battery SOC within the desired window.

\subsubsection{EMC Type II: Global Optimization - DP}

In a SHEV, the engine power profile is determined by an optimization-based EMC while minimizing a cost function for fuel/energy consumption. If the driving cycle is known, meaning that the driver power request is known, then a global optimal solution can be found [66]. While this approach cannot be applied in real-time EMC applications, this method can provide an ideal baseline to assess different EMCs. In this chapter, the DP method is employed for structuring an optimization problem to find optimum strategies for minimizing a performance index. By doing calculation backwards over the time horizon based on Bellman's Principle of Optimality [67], DP searches for the best control action among all the possible actions offline in time by assuming the entire driving cycle information is available.

The engine requested power is the control variable $\left(u_{k}\right)$ and the battery SOC is the 
state variable in this formulation. The control variable is discretized by time step of 1 second. An energy based performance index consisting of the fuel energy and the battery energy is formed in Equation (3.9):

$$
J_{j}\left(t, u_{k}\right)=\underset{j}{\Delta E_{f}}\left(t, u_{k}\right)+\alpha \underset{j}{\Delta E_{b}}\left(t, u_{k}\right)
$$

where, the index $j$ represents the feasible transitions to the next time $(t+1)$ and the index $k$ is the control variable indicator. $k$ is a finite number and its size is equal to the number of possible values for the digitized control variable. In this chapter $k \in[1,2,3,4]$, where $k=1$ represents the engine off situation, and $k=$ 2,3, or 4 represents the engine three selected power levels (modes). Furthermore, $\underset{j}{\Delta E_{f}}\left(t, u_{k}\right)$ and $\underset{j}{\Delta E_{b}}\left(t, u_{k}\right)$ are the energy consumptions of the ICE path and the battery path respectively for the $j^{\text {th }}$ transition between two states. $\alpha$ is an equivalent factor to equate the electrical usage of the battery to virtual fuel consumption. $\alpha$ is a constant number and is sensitive to driving cycles. In this chapter, $\alpha$ is specified offline to enforce the battery to operate in a charge-sustaining mode. $\underset{j}{\Delta E_{f}}\left(t, u_{k}\right)$ and $\underset{j}{\Delta E_{b}}\left(t, u_{k}\right)$ are calculated by the following equations: 


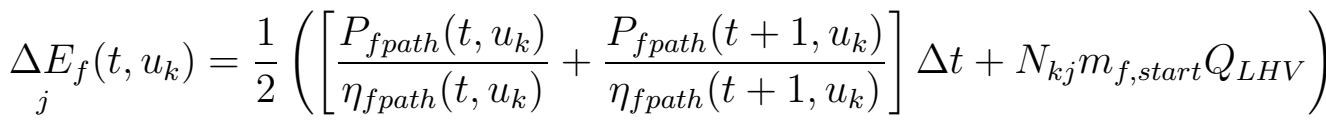

$$
\begin{aligned}
& \Delta E_{j}\left(t, u_{k}\right)=\frac{1}{2}\left[\frac{P_{b p a t h}\left(t, u_{k}\right)}{\eta_{\text {bpath }}\left(t, u_{k}\right)}+\frac{P_{\text {bpath }}\left(t+1, u_{k}\right)}{\eta_{\text {bpath }}\left(t+1, u_{k}\right)}\right] \Delta t
\end{aligned}
$$

where, $\eta_{\text {fpath }}$ and $\eta_{\text {bpath }}$ represent the combined efficiency in the ICE path and the battery path for the transition between the $k^{\text {th }}$ states in the successive times. $P_{b p a t h}$ and $P_{\text {fpath }}$ are the battery power and the engine produced power, respectively. $m_{f, \text { start }}$ in Equation (3.10) is the fuel penalty for each engine start-up. The cost associated with the engine start-up is incorporated in Equation (3.10) by introducing the $N_{k j}$ constant which is equal to 1 when the engine is at start-up and it is equal to 0 during the rest of the engine operation. $Q_{L H V}$ is the gasoline fuel lower heating value. By finding the engine optimum requested power $\left(u_{k}\right)$ to fulfill the driver power request, the battery produced power is calculated according to the following constraint at each state:

$$
P_{\text {bpath }}\left(t, u_{k}\right)=P_{r e q}\left(t, u_{k}\right)-P_{f p a t h}\left(t, u_{k}\right)
$$

In the backward DP, the optimal cost-to-go from the current time $(t)$ to the end of 
the driving cycle is defined as:

$$
S^{*}\left(t, u_{k}\right)=\min _{j}\left[J_{j}\left(t, u_{k}\right)+S^{*}\left(t+1, u_{k}\right)\right]
$$

where, $S^{*}\left(t, u_{k}\right)$ is the optimal cost-to-go from the $k^{t h}$ state at the current time $t$ to the end of the driving cycle. $S\left(t+1, u_{k}\right)$ represents the optimal cost in the next time (i.e., $t+1)$ to the end of the driving cycle. At each time a state $(k)$ which has the minimum cost among the different states is determined by the DP strategy as the optimal control variable. A nonlinear backward HEV model, which does not include the driver model, is used to increase flexibility for the real-time implementation of the optimal EMC model. This backward model assumes that the vehicle tracks exactly the driving cycle; thus, the vehicle power demand is directly calculated from the driving cycle. In addition, a high fidelity forward Simulink vehicle model is designed for assessing the EMC strategy and analyzing the HEV performance. The purpose for including DP results in this chapter is to present the ultimate energy saving using the RCCI hybrid electric powertrain. The DP values serve as a benchmark for the comparison with $\mathrm{RBC}$ and $\mathrm{MPC}$ results with different time horizons. 


\subsubsection{EMC Type III: MPC}

The MPC concept is deployed to form the EMC optimization problem on a moving receding horizon. Given that the vehicle speed can be predicted over a short Time Horizon (TH), the DP strategy is used over a short horizon to find a sequence of the sub-optimal control strategy. The MPC strategy is developed by assuming that the future driving cycle information over the TH is provided from the GPS data. The

cost function $(J)$ at the $n^{\text {th }} \mathrm{TH}$ is shown in Equation (3.14). $J$ is minimized over TH by selecting optimal ICE/generator power request.

$$
J(n)=\int_{t_{n}}^{t_{n}+T H}\left(E_{f p a t h}+\alpha \cdot E_{b p a t h}\right) d t
$$

subject to:

$$
\begin{array}{r}
P_{e, \text { min }}<P_{e}<P_{e, \text { max }} \\
P_{m, \text { min }}<P_{m}<P_{m, \max } \\
N_{e, \min }<N_{e}<N_{e, \max } \\
N_{m, \min }<N_{m}<N_{m, \max } \\
S O C_{\min }<S O C<S O C_{\max } \\
S O C_{@} t=0=0.8
\end{array}
$$


where, the subscripts $\max$ and min denote maximum and minimum, respectively. The first term in Equation (3.14) refers to the fuel energy consumed by the ICE and the second term refers to the battery electrical energy consumed or recharged during the driving cycle. The subscripts $e$ and $m$ in Equation (3.15) denote engine and motor, respectively. The DP formulation in Section 3.4 .2 is used over the TH to calculate the optimal ICE/generator power at time step $n$. A closed-loop MPC is designed to reject disturbances such as sudden changes in the estimated driving cycle data. However, this EMC strategy does not provide a globally optimal solution, but it can be used for real-time implementation. In the current formulation, the time domain is discretized into one-second intervals. The solutions consist of local optimum control signal at each time step.

\subsection{Results and Discussions}

In this section the results for the SHEV with the RCCI, CI, and SI engines are discussed. In subsection 3.5.1, sensitivity of the driving cycle's prediction time horizon on the vehicle's fuel economy is studied. Moreover, the initial battery SOC effect on the vehicle's fuel economy is investigated in subsection 3.5.2, Lastly, the effect of the type of the driving cycle is presented in subsection 3.5.3.

In this chapter a combined driving cycle (Figure 3.7) consisting of three standard driving cycles including UDDS (Urban Dynamometer Driving Schedule), HWFET 
(Highway Fuel Economy Test), and US06 is used to test the EMC strategies. The combined driving cycle is the base driving cycle for all the analysis in this chapter except for subsection 3.5.3, where different driving cycles are compared.

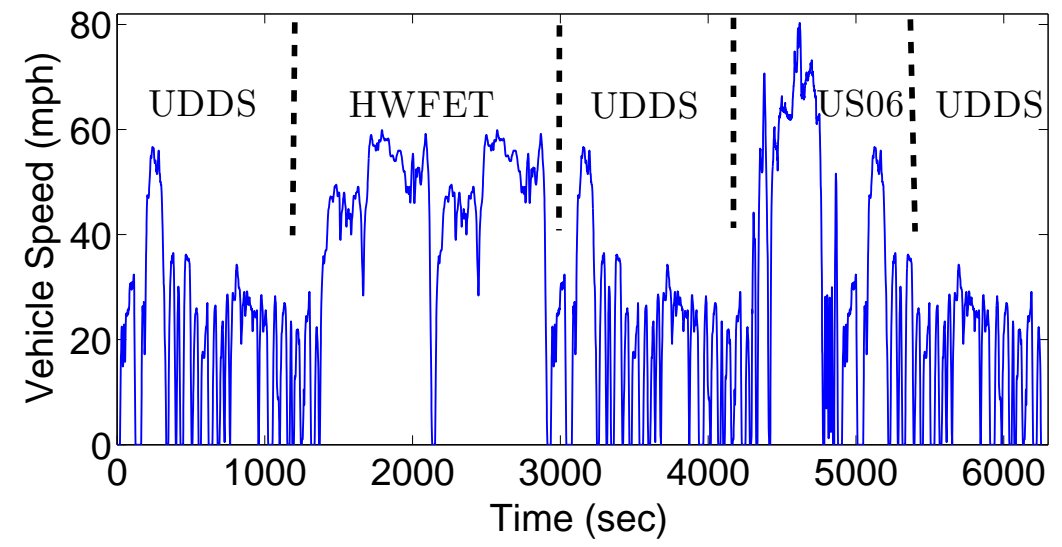

Figure 3.7: The combined driving cycle used for the evaluation of the designed EMCs.

Figure 3.8 describes the effect of the engine start-up fuel penalty $\left(m_{f, s t a r t}\right)$ on the fuel saving. The RCCI fuel economy improvement is constant versus the amount of $m_{f, \text { start }}$. This is because the power levels for each engine are similar; thus, the number of engine on/off switching will remain constant. This makes the RCCI fuel economy improvement independent of the $m_{f, s t a r t}$ value. Figure 3.8 also shows the same trend for the RCCI fuel saving over the number of engine operating points and the fuel economy improves with increasing number of the engine operating points. 
a) Improvement over SI

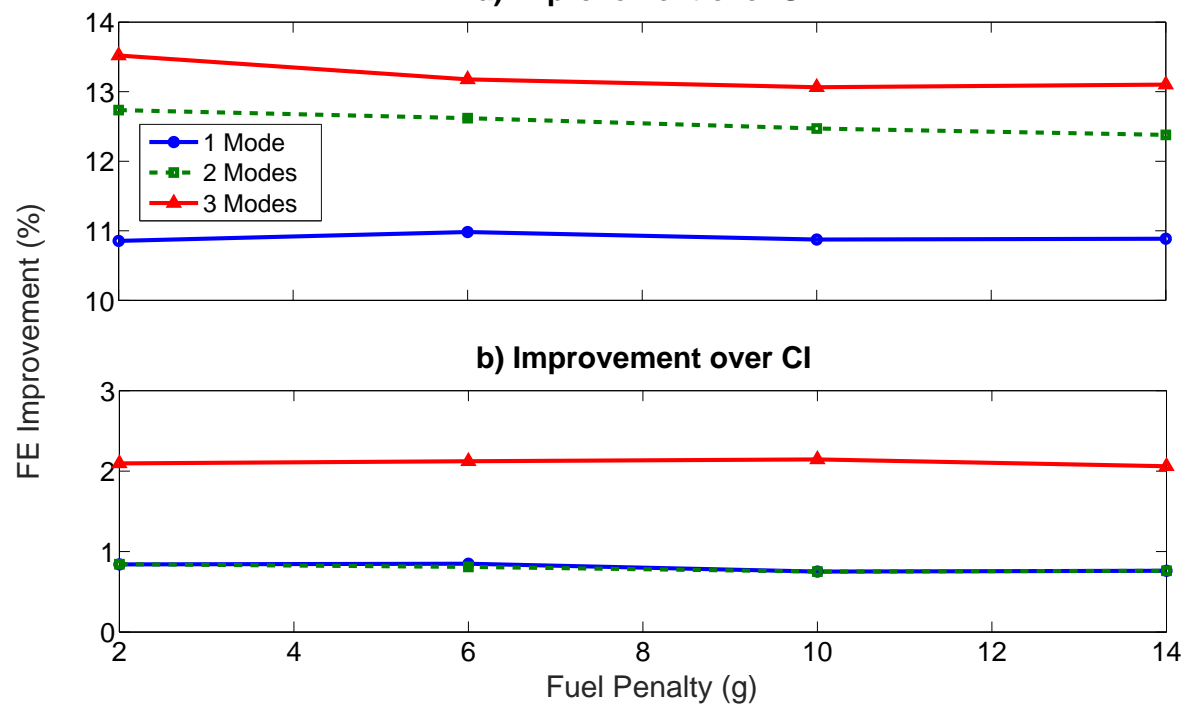

Figure 3.8: Fuel economy (FE) improvement by using RCCI versus a) SI and b) CI engines as a function of number of engine operating points and engine start-up fuel penalty. (EMC: DP)

Figure 3.9 illustrates the E-motor speed variation for the combined driving cycle. A single gear is designed to synchronize the E-motor and vehicle maximum speeds in order to expand the E-motor operating points to the whole E-motor operating region. In Figure 3.9, for the portion of the US06 driving cycle that the vehicle speed reaches to $80 \mathrm{mph}$, the E-motor speed is $6000 \mathrm{rpm}$. 


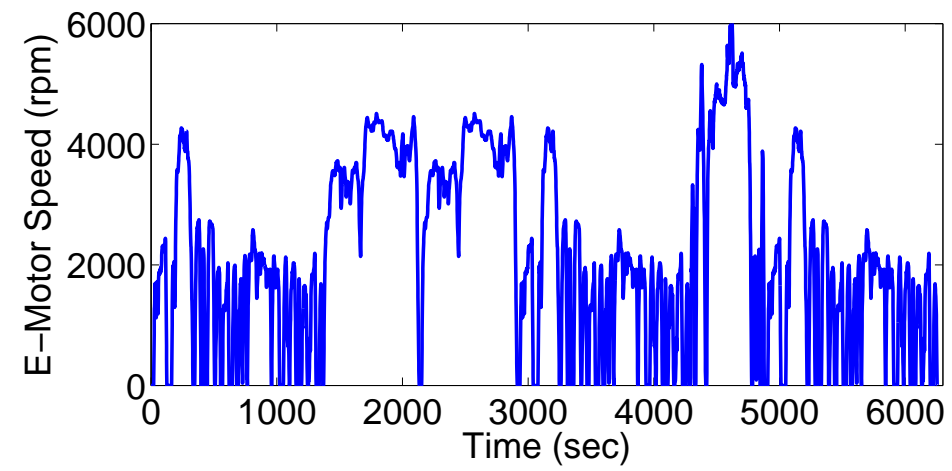

Figure 3.9: Traction E-motor speed during the combined driving cycle.

The E-motor operating points during the combined driving cycle are depicted in Figure 3.10 . The E-motor efficiency points range from $70 \%$ to $90 \%$ in the traction and regenerative braking modes. The E-motor efficiency is higher at higher E-motor power (i.e., $P_{e}>40 \mathrm{~kW}$ ) and the E-motor efficiency improves with increasing the E-motor power. 


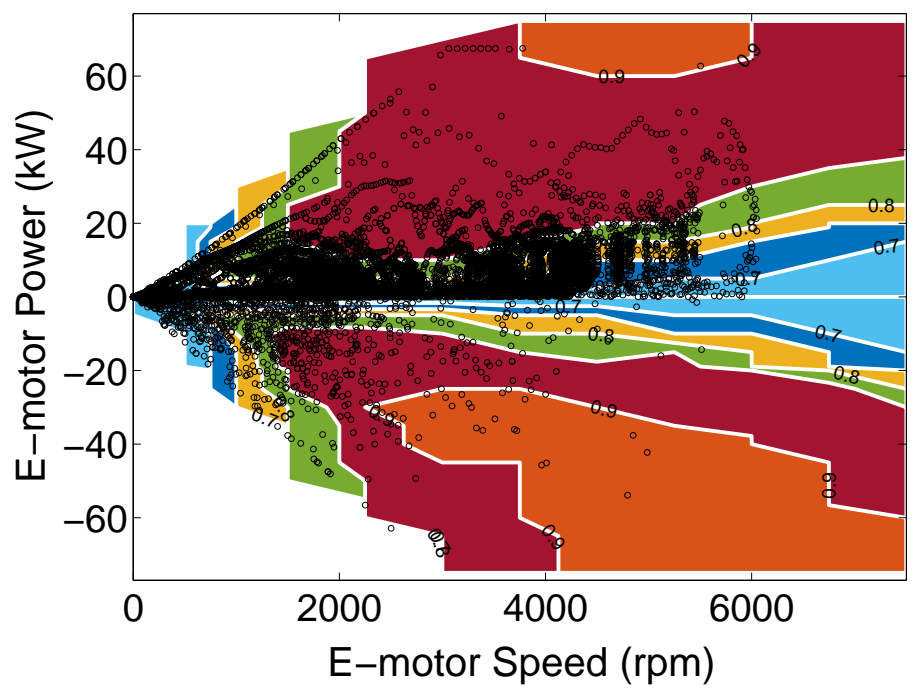

Figure 3.10: E-motor operating points, shown with ' $o$ ' symbol, plotted over the E-motor efficiency map.

Figure 3.11 shows the vehicle tracking performance. The vehicle is able to follow the reference driving cycle with Root Mean Square Error (RMSE) of $0.8 \mathrm{mph}$. This result also confirms that the HEV components' sizing meets the performance requirements during the driving cycle. Thus, the HEV model can be used as a testbed for evaluation of EMCs. 


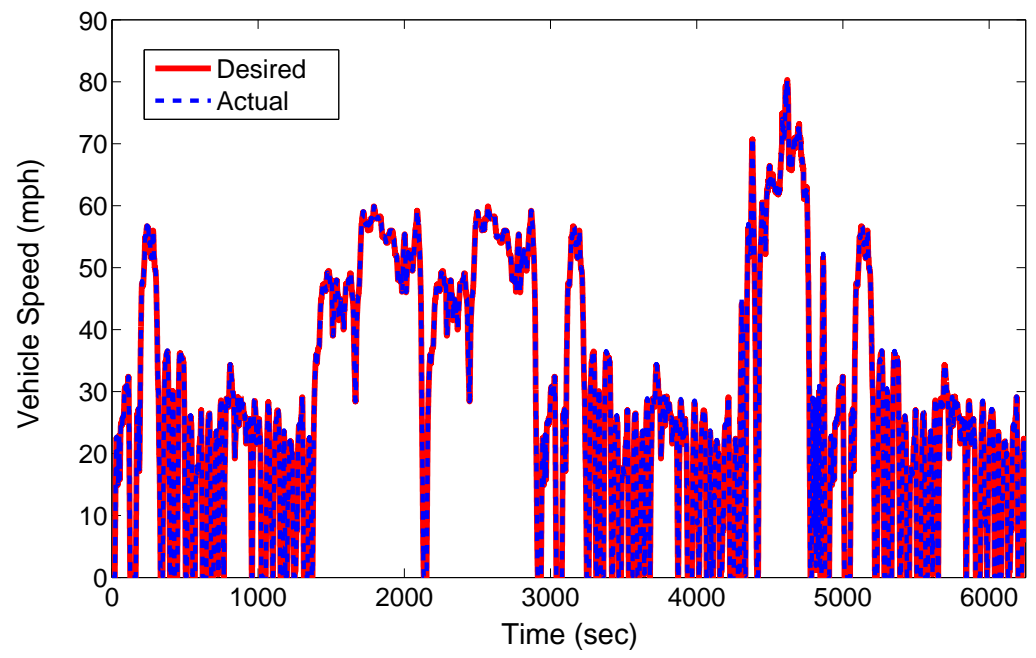

Figure 3.11: Desired vehicle speed vs. actual vehicle speed with root mean square tracking error of $0.8 \mathrm{mph}$.

Figure 3.12 compares the engine on/off status and the battery SOC variation for both RBC and DP EMCs. The RBC strategy regulates the engine status command based on the SOC value. The RBC switches the engine on when the SOC reaches to its lowest allowed value (i.e., $S O C_{\min }=0.55$ ). In the $\mathrm{DP}$ controller, the $\mathrm{SOC}$ variation is much less than that of the RBC. This allows the EMC to choose the most optimal control strategy over the driving cycle at each time. However, in the DP strategy the engine switches on and off more frequently (Figure 3.12 -b). 


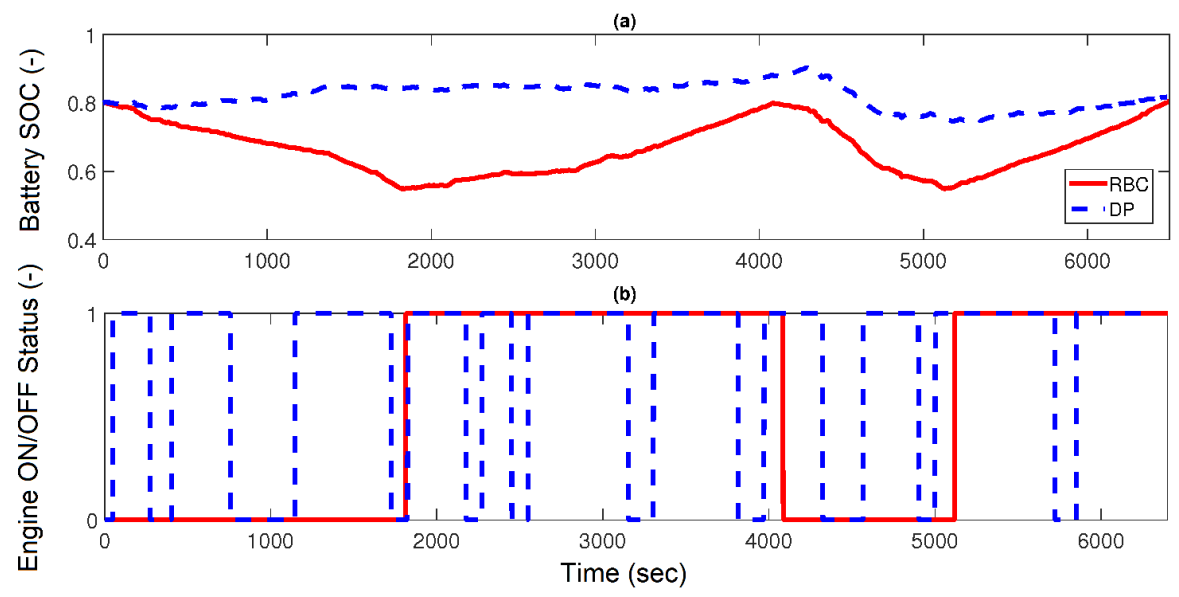

Figure 3.12: Engine on/off status and the battery SOC pattern in the 3-mode DP and RBC strategies.

Figure 3.13 indicates the power distribution of the battery and the engine during the driving cycle for the DP control strategy. There are different operating modes such as fully electric mode and opportunity charging mode based on Figure 3.13. For instance, the battery only supplies the driver power demand at $\mathrm{t}=1282 \mathrm{sec}$ and runs the vehicle on the fully electric mode. For low power demands, at $t=3005 \mathrm{sec}$, the vehicle runs in the opportunity charging mode, in which the engine/generator supplies the power demand and charges the battery simultaneously. In $\mathrm{RBC}$, the engine is turned off during the braking, but in the DP strategy the engine can either be on or off to minimize the cost function. In addition, at the low vehicle speeds the mechanical braking assists the regenerating braking to supply the braking torque; however, at the high vehicle speeds all the braking torque is supplied by the regenerative braking. 


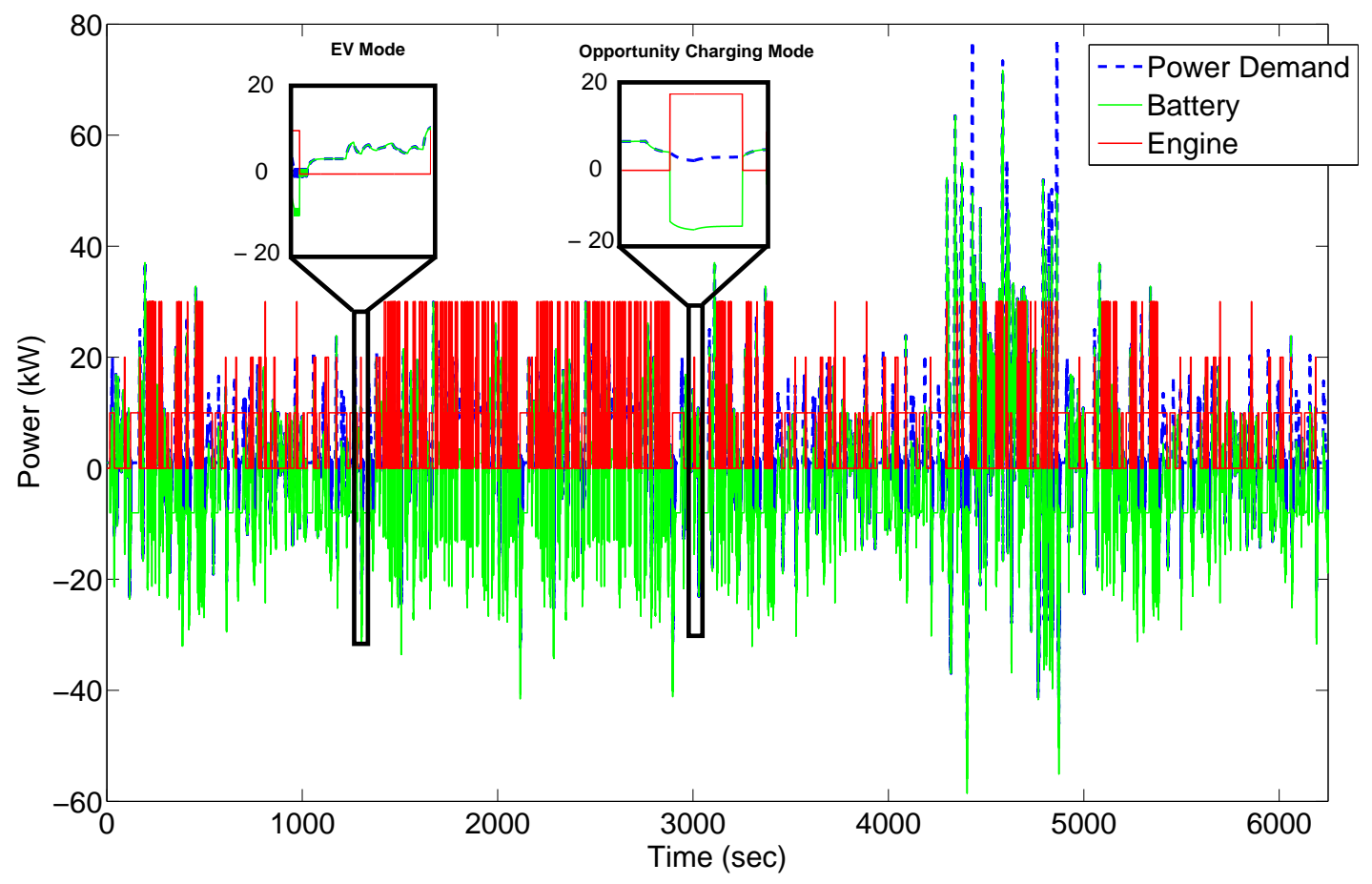

Figure 3.13: Power distribution in the SHEV during the combined driving cycle. (EMC: 3-mode DP)

The SOC variations over the driving cycle for the DP and MPC controllers are illustrated in Figure 3.14. The EMC is in the charge-sustaining mode and the EMC strategy is tuned to enforce the final SOC value to match the initial SOC value at the end of the driving cycle. Moreover, Figure 3.14 compares the SOC profile for each EMC. The SOC variation for different prediction TH is also shown. The results in Figure 3.14 show that all the designed EMCs can sustain SOC at the initial SOC by the end of the driving cycle. The designed DP EMC algorithm in this chapter is computationally efficient. The simulation processing time on a $2.20 \mathrm{GHz}$ Intel processor is about 72.6 seconds for the whole combined driving cycle (i.e., $11.5 \mathrm{~ms}$ per 
one second of the driving cycle).

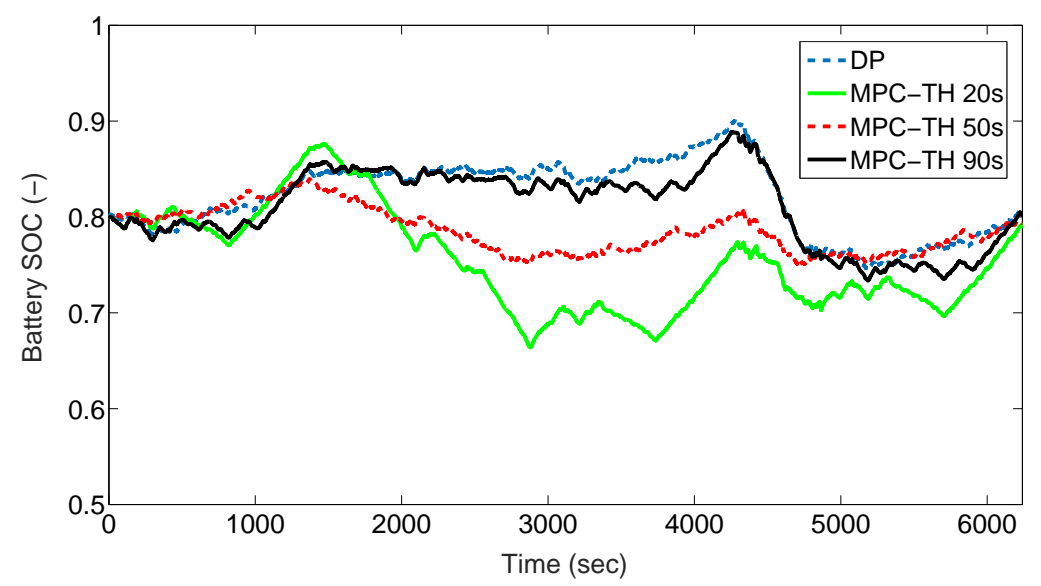

Figure 3.14: Battery SOC variations in MPC and DP controllers. (EMC: 3 -mode)

\subsubsection{Prediction Time Horizon Sensitivity}

Figure 3.15 demonstrates the HEV fuel economy sensitivity with the driving cycle prediction $\mathrm{TH}$ for different engines. It is observed that the fuel economy increases by knowing more driving cycle information (i.e., larger $\mathrm{TH}$ ) and it reaches a plateau, in which at $\mathrm{TH}=120 \mathrm{sec}$ the $\mathrm{HEV}$ fuel economy merges to the DP results. There is a trade-off between the computational cost and fuel saving by the MPC strategy. Higher TH improves the HEV fuel economy at the cost of need for more computation time. In these simulations, $\mathrm{TH}=70$ sec offers a good compromise between computation load and fuel economy improvement. This results show by knowing a short time horizon information of the vehicle's speed profile (i.e., TH=70 sec), 95\% of the global optimal 
fuel economy (i.e., DP) is achieved. This figure also compares different engines' fuel economy variation with the EMC types. The RCCI engine offers the highest fuel economy compared to SI and CI engines. In the DP EMC, the RCCI engine has 5.9 and 1.1 MPG greater fuel economy than the SI and CI engines, respectively.

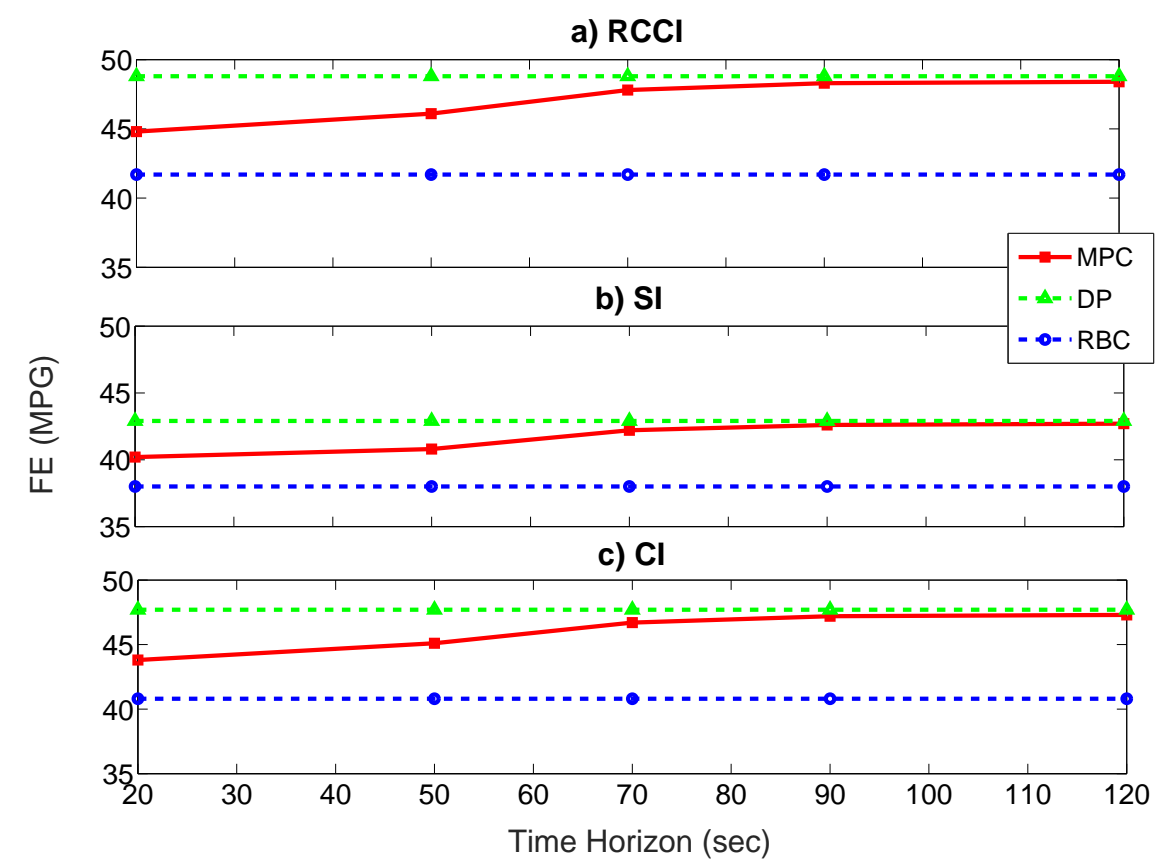

Figure 3.15: Fuel economy (FE) comparison for a) RCCI, b) SI, and c) CI engines for different EMCs.

\subsubsection{Initial SOC Sensitivity}

In this section, the effect of running the RCCI engine in a lower initial SOC on the fuel economy is studied. The low SOC can represent operation in Extended Range Electric Vehicle (E-REV). It is assumed the battery is operating at a lower initial 
SOC value (i.e., $\left.S O C_{\text {initial }}=0.3\right)$ during the charge-sustaining mode. Fuel economy of the HEV integrated with RCCI, SI, and CI engines for different EMCs are shown in Table 3.5.

Table 3.5

Fuel economy comparison for different EMCs including the effect of MPC Time Horizon (TH) in the SHEV with $S O C_{\text {initial }}=0.3$. (EMC: 3-mode)

\begin{tabular}{lccc}
\hline \hline $\begin{array}{c}\text { Energy Management } \\
\text { Controller }\end{array}$ & \multicolumn{3}{c}{ Fuel Consumption (MPG) } \\
\cline { 2 - 4 } & RCCI & SI & CI \\
\hline On-off RBC & 41.4 & 37.4 & 40.0 \\
MPC -TH20s & 44.4 & 39.2 & 43.0 \\
MPC -TH50s & 45.7 & 40.3 & 44.2 \\
MPC -TH70s & 47.4 & 41.7 & 45.8 \\
MPC -TH90s & 47.9 & 42.1 & 46.2 \\
MPC -TH120s & 48.0 & 42.2 & 46.3 \\
DP & 48.4 & 42.3 & 46.7 \\
\hline \hline
\end{tabular}

Comparing Figure 3.15 and Table 3.5 shows that the fuel economy is greater when the $S O C_{\text {initial }}$ is higher. In lower $S O C_{\text {initial }}$ scenario, the battery has greater losses than that in higher initial SOC since the battery loss is greater in the low SOC region. Figure 3.16 shows the battery energy losses in the both scenarios. It can be seen that the SHEV with lower initial SOC has $31 \mathrm{~kJ}$ more battery energy losses than the larger SOC case in the combined driving cycle. The battery energy losses should be compensated by running the ICE for a longer time, which leads to lower fuel economy in the low $S O C_{\text {initial }}$ scenario.

Figure 3.17 shows the engine produced power profile in both low $S O C_{\text {initial }}$ SOC and high $S O C_{\text {initial }}$ scenarios. The number of ICE on/off switching is identical in 


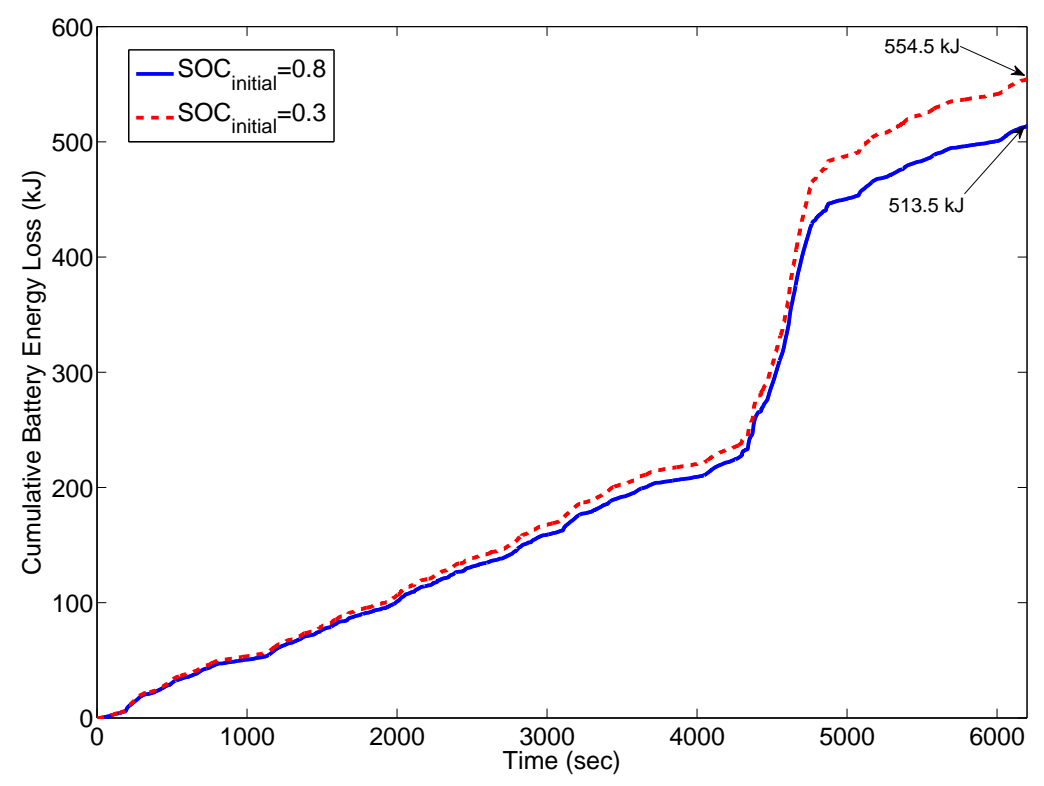

Figure 3.16: Comparison between battery energy loss for the low $S O C_{\text {initial }}$ and high $S O C_{\text {initial }}$ operating conditions. (EMC: 3-mode DP)

the two cases, but the engine runs for a longer time in the low initial SOC case. Therefore, the RCCI engine in this condition has more opportunity to work and consequently more fuel saving is achieved compared to the SI and CI engines. This is shown in Figure 3.18, where the SHEV with low $S O C_{\text {initial }}$ has higher fuel economy improvement 2 compared to the high $S O C_{\text {initial }}$ scenario.

\footnotetext{
${ }^{2}$ Fuel economy improvement is calculated by $\frac{F E_{H E V-R C C I}-F E_{H E V}}{F E_{H E V}} \times 100$ where $F E_{H E V-R C C I}$ is the fuel economy for the RCCI-HEV powertrain, and $F E_{H E V}$ is the fuel economy for SI-HEV or CI-HEV powertrain.
} 


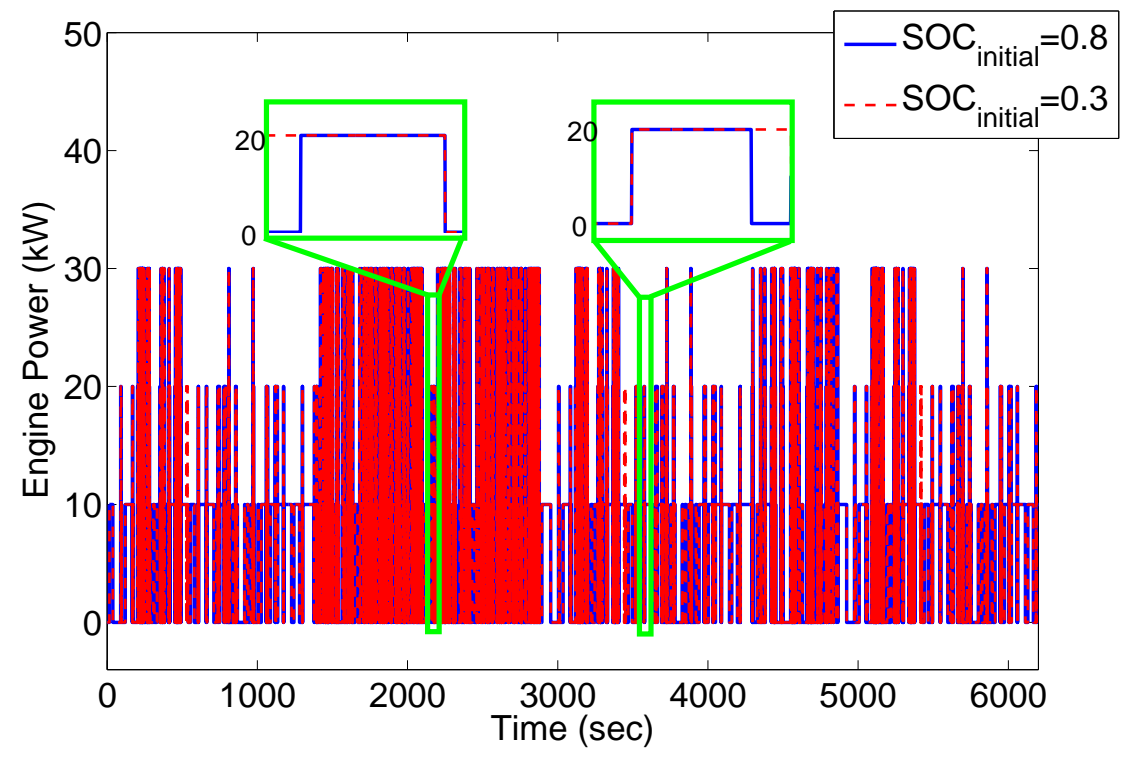

Figure 3.17: The RCCI engine power pattern in the SHEV for two initial SOC operating conditions. (EMC: 3-mode DP)

(a) Improvement over SI

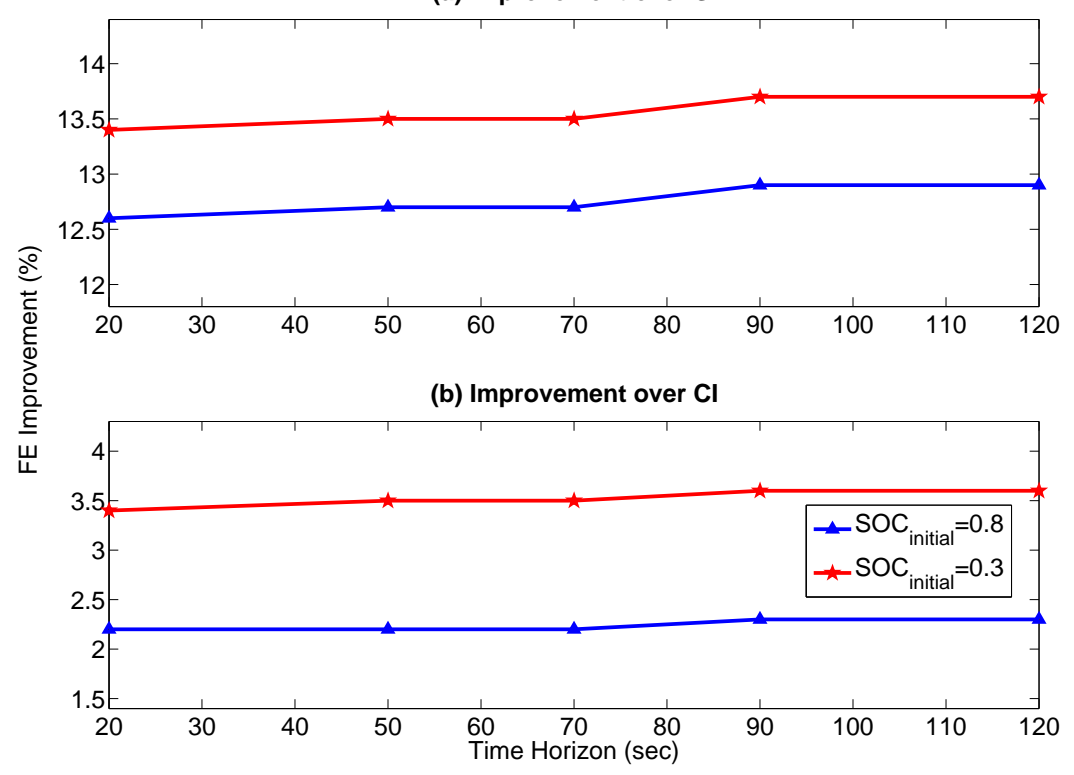

Figure 3.18: Fuel economy (FE) improvement by using the RCCI engine over the SI or CI at two initial SOC operating conditions. (EMC: 3-mode MPC) 


\subsubsection{Driving Cycle Effect}

Here the effect of using various driving cycles is investigated for the RCCI fuel economy improvement for both SHEVs with low initial SOC and high initial SOC scenarios. Four different driving cycles including US06, HWFET, UDDS, and the combined driving cycle are studied. Figure 3.19 shows the effect of the various driving cycles on the HEV fuel economy improvement by incorporating the RCCI engine versus conventional SI and CI engines. The fuel economy improvement of the RCCI engine versus the SI engine varies from $13.1 \%$ to $14.2 \%$ depending on driving cycles. Moreover, the fuel economy improvement versus the CI engine varies from $1.8 \%$ to $3.0 \%$.

Table 3.6 lists the required average power of the HEVs in each of the studied driving cycles. By comparing Table 3.6 and Figure 3.19, it becomes clear that the average power of a driving cycle has a direct effect on the RCCI fuel economy saving over the SI and CI engines. In high power demand driving cycles (e.g., US06), the engine needs to run longer for charging the battery and compensate for the higher power demand. This gives the RCCI engine more opportunities to save more fuel since it is generally more fuel-efficient than conventional SI and CI engines. In addition, the initial SOC effect in Figure 3.19 is related to the difference in the length of the engine runtime, as previously mentioned in subsection 3.5 .2 . 


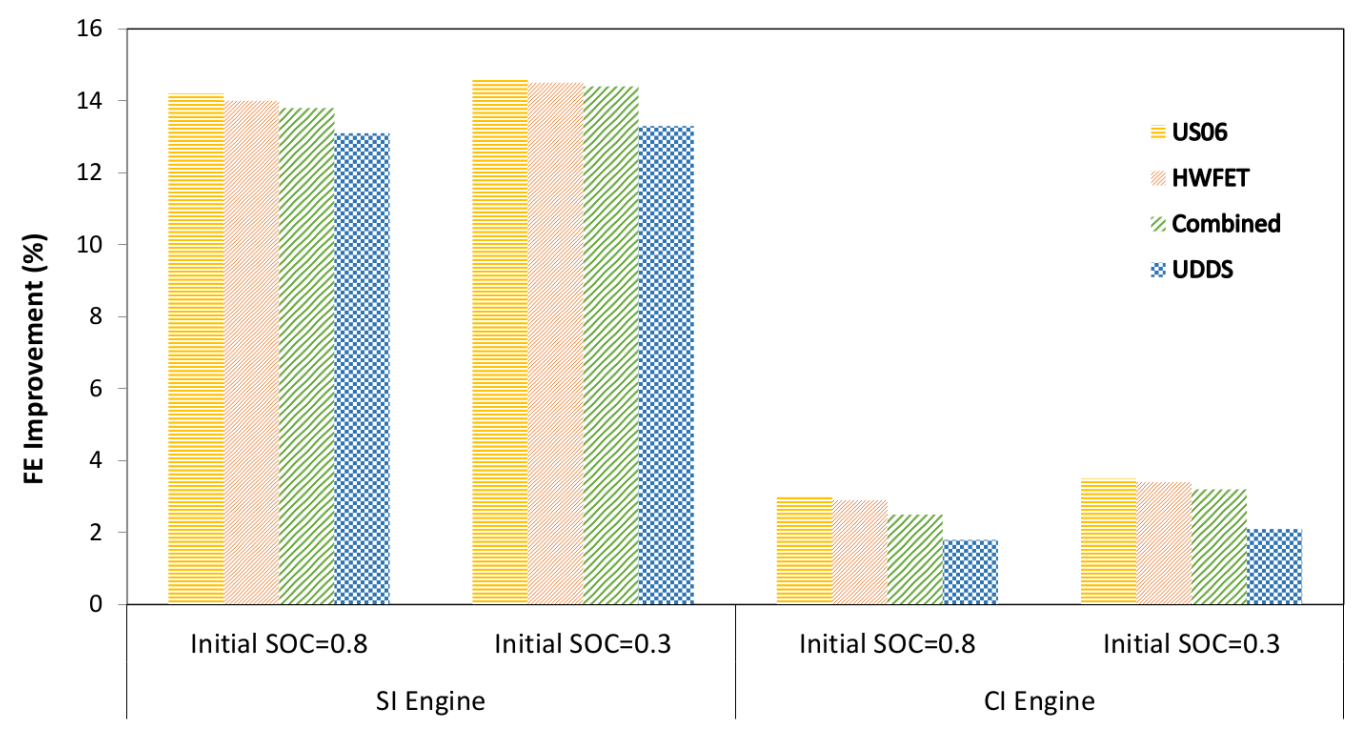

Figure 3.19: HEV fuel economy (FE) improvement by using the RCCI engine versus the SI and CI engines in four different driving cycles. (EMC: 3-mode DP)

Table 3.6

Average power in the studied driving cycles.

\begin{tabular}{lc}
\hline \hline Driving Cycles & Average Base Power (kW) \\
\hline US06 & 19.1 \\
HWFET & 12.0 \\
Combined & 8.2 \\
UDDS & 5.2 \\
\hline \hline
\end{tabular}

\subsection{Conclusions}

In this chapter, an RCCI engine was integrated with SHEV powertrain. The fuel economy benefit of the RCCI-HEV powertrain was compared with the conventional ICE-HEV powertrains. Three different ICEs were studied including a GM Z19DTH 
engine in CI and RCCI modes and a GM A14XFL SI (gasoline) engine. For a meaningful comparison among the different powertrains, the ICE produced the same amount of power for each powertrain. In selecting engine operating points the engine-out emission constraints were considered. A high-fidelity forward-in-power SHEV model was developed in Matlab ${ }^{\circledR} /$ Simulink with experimentally validated submodels. Three different types of EMC strategies including RBC, DP, and MPC were developed to investigate the effect of the control strategy on the potential fuel saving from an RCCI-based HEV. The following summarizes the main findings from this chapter:

$\dagger$ The simulation results showed the RCCI engine offers significant potential for fuel saving in SHEV architecture. In the combined driving cycle, integrating an RCCI engine with a SHEV powertrain provided up to $12.6 \%$ higher fuel economy over a modern SI engine, while the improvement over the CI-based HEV was $2.2 \%$ for the combined driving cycle.

$\dagger$ Increasing number of the engine operating points can increase the RCCI-HEV fuel economy (FE) improvement by $2.1 \%$ by utilizing more engine operating points. This chapter did not consider fuel penalty during ICE transients between engine modes. It is anticipated that too frequent mode switching is not desirable for fuel saving once a large number of engine operating points is used.

$\dagger$ The 3-mode DP provides the best FE improvement among the EMCs studied. Using the 3-mode DP leads to $17.0 \%$ more FE improvement compared to 
the RBC EMC. This demonstrates the importance of designing optimal EMC strategies to maximize the FE improvement of the RCCI-HEV.

$\dagger$ In the MPC strategy, more available information from the driving cycle (larger prediction time horizon) naturally leads to better fuel economy improvement and the MPC results eventually merge to the DP optimal controller results. Prediction horizon length of 70 seconds showed a compromise between the computational cost and fuel economy improvement in this chapter.

$\dagger$ Among the four driving cycles studied, the driving cycle that has higher average power $(\bar{P})$ has higher RCCI fuel saving. Thus, the RCCI-HEV operating in US06 driving cycle $(\bar{P}=19.1 \mathrm{~kW})$ offered $14.2 \%$ fuel economy improvement versus $13.1 \%$ in UDDS driving cycle $(\bar{P}=5.2 \mathrm{~kW})$ in the SHEV. This was because the engine needs to run for a longer time to compensate for the higher power demand. This leads to more opportunity for the RCCI engine to save more fuel in compared to the SI and CI engines.

$\dagger$ RCCI-SHEV operation with battery at low SOC in charge-sustaining mode resulted in slightly higher fuel economy improvement for the RCCI engine in comparison to a higher initial SOC scenario. This is because the battery in low SOC region has larger energy loss which means the engine requires to run for a longer time to compensate for the higher battery losses. This provides more opportunity for the RCCI engine to save more fuel in comparison to the SI and CI engines. 


\section{Chapter 4}

\section{Hybridized Multi-Mode LTC-SI Engine Experimental Setup 1}

Chapter 4 of this dissertation includes design of a test setup for hybrid electric powertrain integrated with a multi-mode LTC engine. The test setup consists of a 465 hp double-ended $\mathrm{AC}$ dynamometer which one side of the dyno is connected to the multi-mode LTC engine, and its other side is coupled with the electric powertrain. This architecture allows the engine and e-motor torques to be blended to meet the torque demand on the dynamometer. The multi-mode engine test setup development was started in January 2013 by prior graduate students in Energy Mechatronics

\footnotetext{
${ }^{1}$ The results in this chapter are partially based on [3] with permission from Elsevier as shown in Appendix D
} 
Laboratory (EML) and details of the engine setup development are found in references [9, 85, 86, 87, 88]. Moreover, designing and building the electric powertrain test setup started in May 2014 and the setup has been already completed. The test setup is capable of implementing different EMC strategies for different levels of hybridization.

Figure 4.1 shows the experimental setup developed for this study. The engine is fuel-flexible and it operates in different LTC modes including HCCI, RCCI and also conventional SI mode. The electric powertrain is capable of realizing different levels of powertrain electrification.

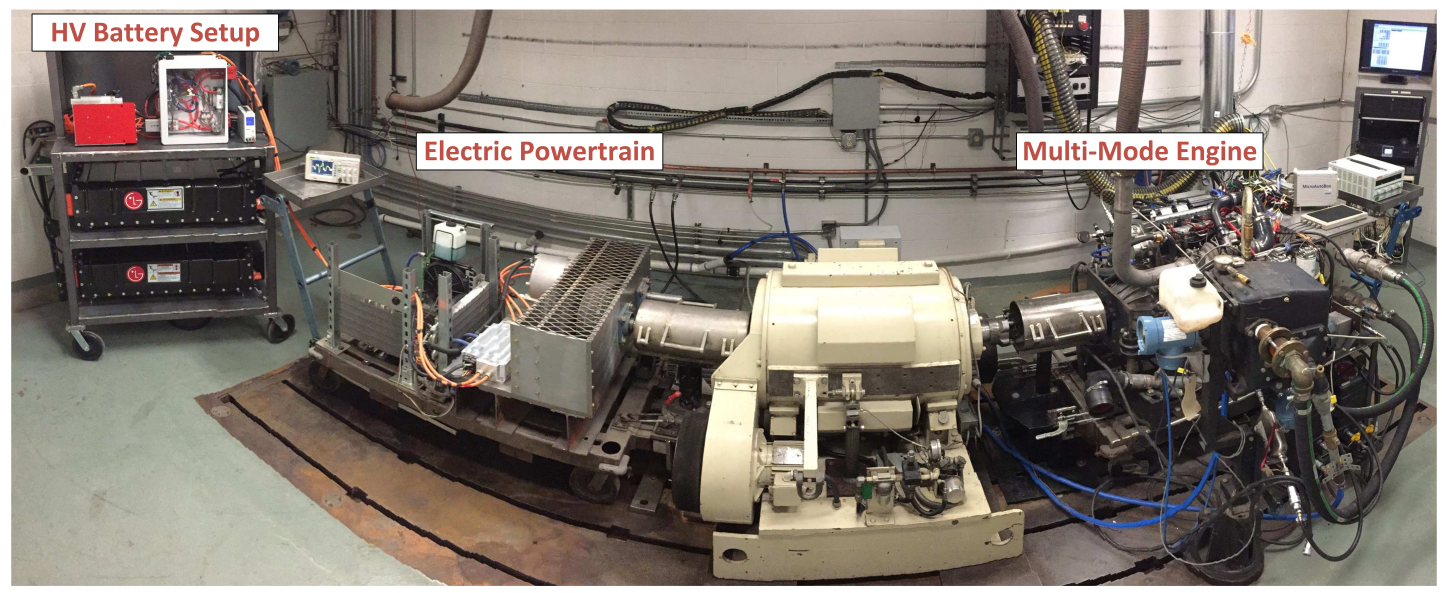

Figure 4.1: Developed LTC-based hybrid electric powertrain experimental testbed with a double-ended $465 \mathrm{hp}$ AC dynamometer at Michigan Technological University.

In this chapter, first the electric powertrain setup with different components are introduced. The details of the setup hardware and software developments are presented in Appendix B. Here, the multi-mode LTC-SI engine setup is briefly introduced and 
the engine required data for the Chapters [5] and [6] are presented.

\subsection{Electric Powertrain}

The design of the electric powertrain started with CAD modeling. The powertrain is designed to be on a portable cart due to the test setup constraints. Figure 4.2 shows the final CAD model setup for integrating the e-motor to the dynamometer. The e-motor is connected to the dyno shaft through a pulley with 2 to 1 ratio. The e-motor and inverter cooling systems are designed to be on the backside of the cart.

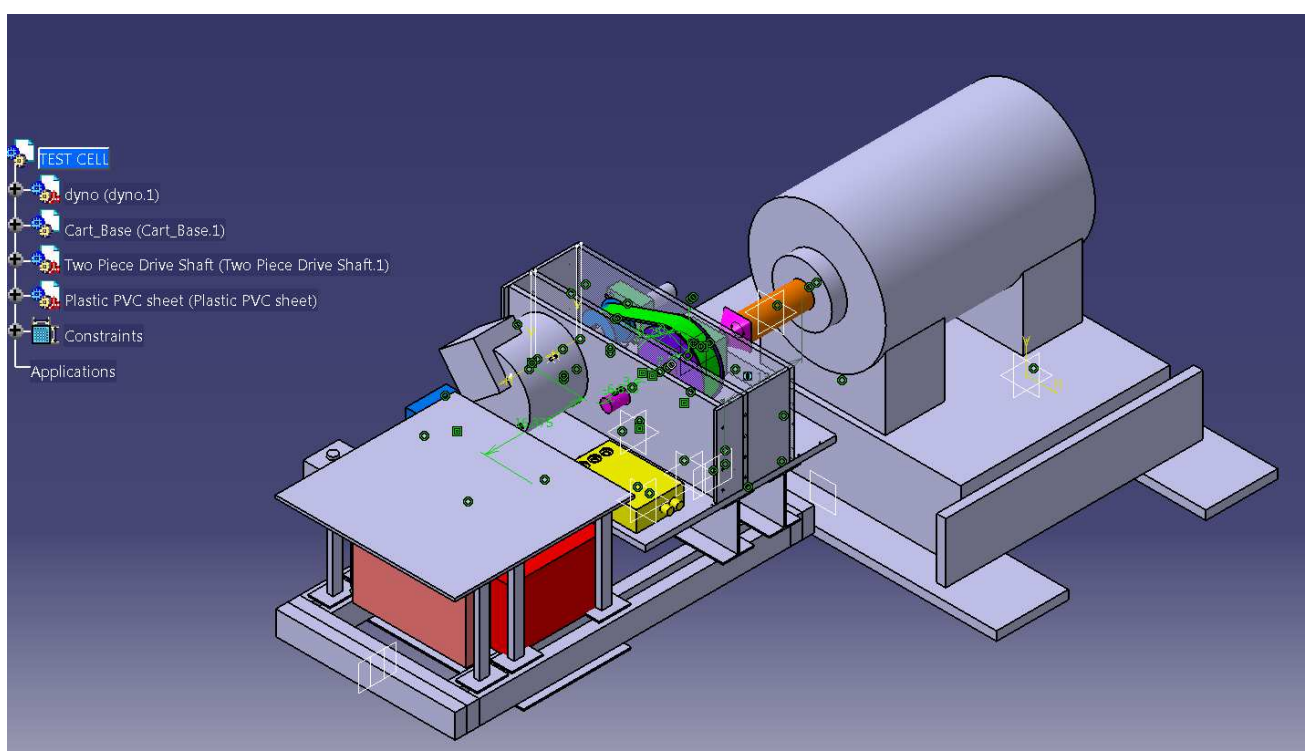

Figure 4.2: CAD model of the e-motor test setup in CATIA.

To develop the LTC-HEV test setup, different parts were needed to be designed and built: 
$\dagger$ Design and fabrication of a mount for coupling the e-motor and the drivetrain to connect the e-motor to the dynamometer.

$\dagger$ Design and fabrication of the cooling systems for electric subsystems including the e-motor and the inverter.

$\dagger$ Design and building a high voltage setup for connecting High Voltage (HV) batteries to the e-motor setup.

$\dagger$ Design and building safety features including a pre-charge circuit for enabling the inverter.

$\dagger$ Development of harness diagrams for subsystems wiring.

$\dagger$ Development of model-based supervisory control software interface for LTC electrified powertrain testing using MicroAutoBox (MABX) dSPACE.

$\dagger$ Building a CAN based hardware setup to interact with sub-systems.

$\dagger$ Implementation of battery charging hardware and software interfaces for the battery charging. This battery charging station enables experiments for the Plug-in HEV (PHEVs) scenarios.

To design the e-motor mount, stress analysis was conducted in the ANSYS (Figure 4.3). The purpose of the stress analysis was to select the size and the materials of the components such as the drivetrain shafts, the mount plates, and the base I-beams. 


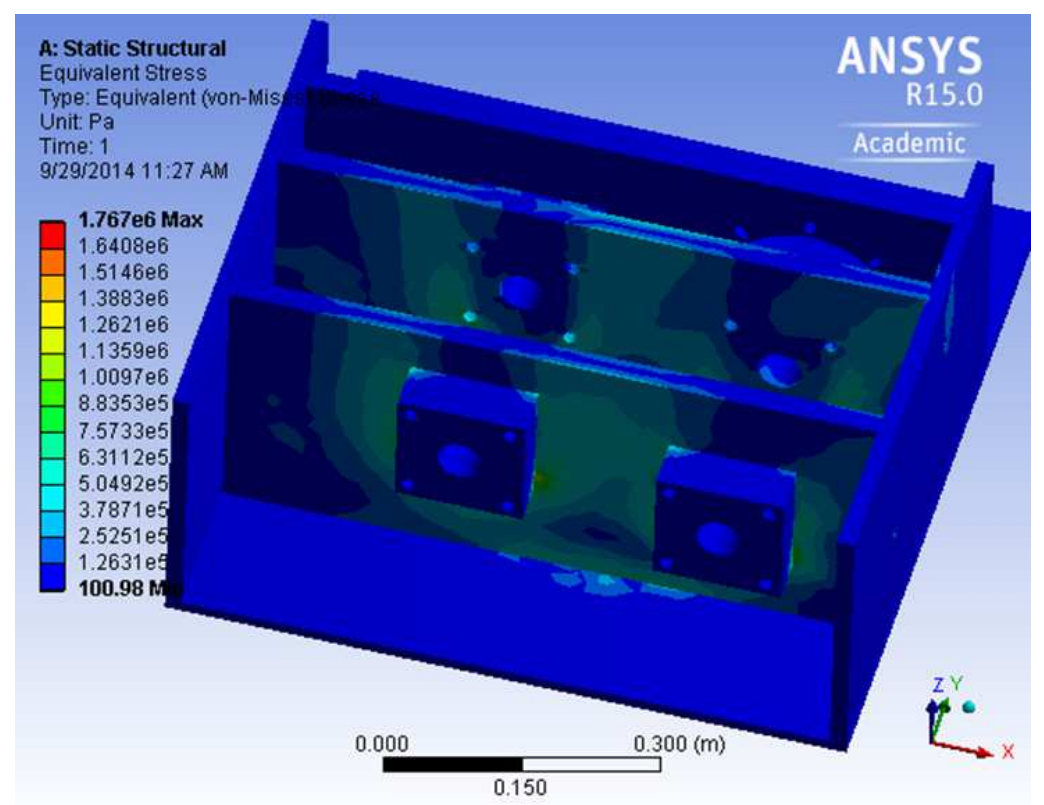

Figure 4.3: Stress analysis in ANSYS for the e-motor mount design.

Figure 4.4illustrates an overview of the test setup. The test setup runs with a $100 \mathrm{~kW}$ synchronous induction Remy motor, which is controlled by RMS PM100DX inverter (more details in subsection 4.2). Two LG Chem batteries are used to supply the electric energy for the electric motor (see table 4.1).

Table 4.1

Battery specifications.

\begin{tabular}{lc}
\hline \hline Parameters & Values \\
\hline Energy Capacity (kWh) & 5 \\
Maximum Voltage (V) & 410 \\
Nominal Voltage (V) & 360 \\
Minimum Voltage (V) & 260 \\
SOC Operating Range (\%) & $30-70$ \\
Battery Pack Mass (kg) & 90 \\
\hline \hline
\end{tabular}

In addition, to safely enable the inverter, an HV box was designed and a pre-charge circuit was designed to control the current flow to the inverter. Moreover, the designed 
HV box disconnects the battery from the setup in the case of emergency. The supervisory controller was implemented in MicroAutoBox II (MABxII) dSPACE 1512. The control strategy was developed in Matlab/Simulink to control the sub-systems. For running the test setup, a program was designed in StateFlow to conduct the components sequence scheduling.

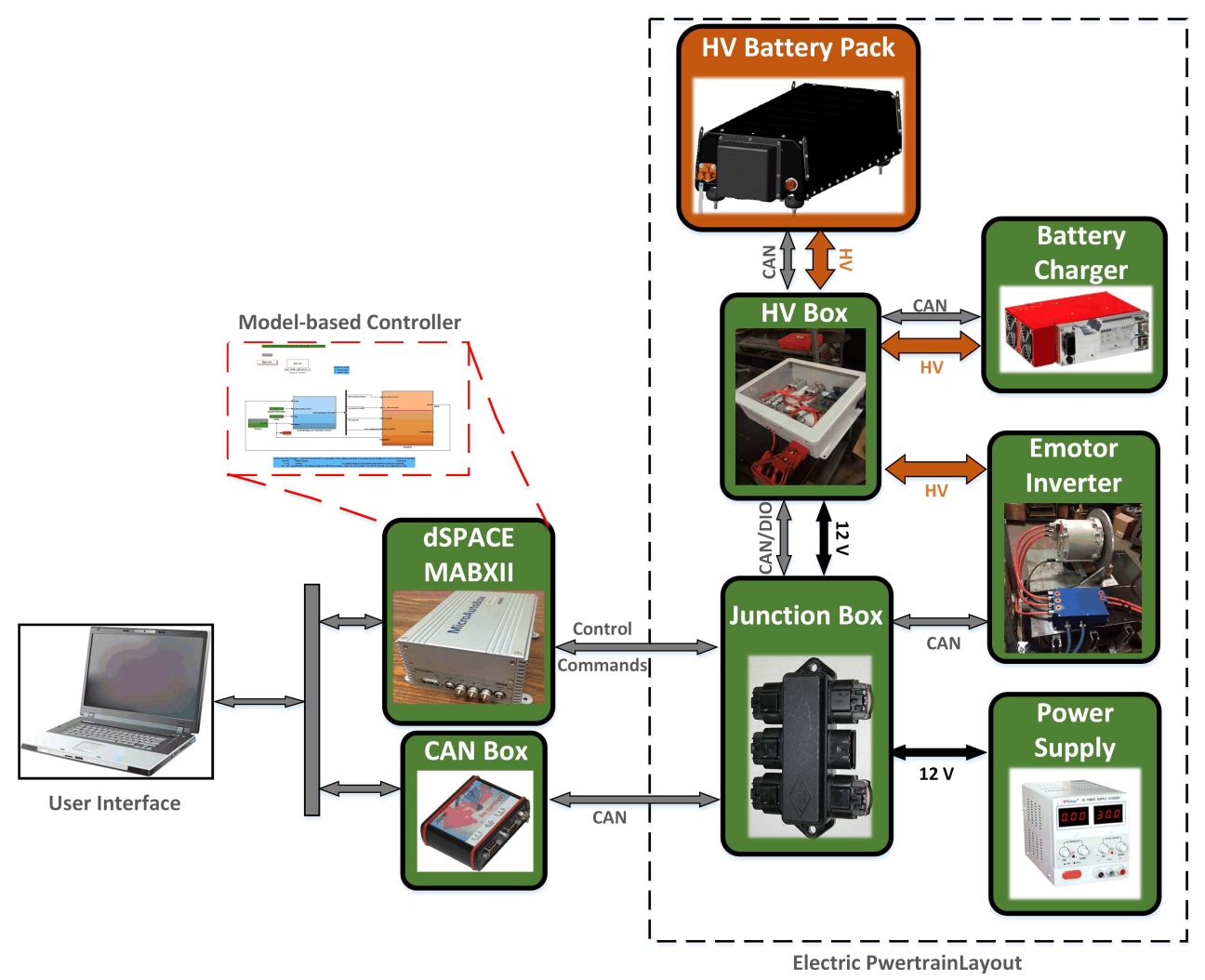

Figure 4.4: Electric powertrain components layout of the LTC-HEV testbed 


\subsection{E-Motor/Inverter}

The e-motor is made by Remy company and the model is HVH250-090-SOM. The e-motor specifications and operating conditions are listed in Table 4.2.

\section{Table 4.2}

E-motor specifications.

\begin{tabular}{lc}
\hline \hline Items & Descriptions \\
\hline DC Bus Voltage Maximum (V) & 700 \\
Peak Current (Arms) & 300 \\
Rated Peak Operating Time (sec) & 60 \\
Coolant Inlet Temperature (C) & 90 \\
Mass (kg) & 49 \\
Rotational Inertia $\left(\mathrm{kg}-\mathrm{m}^{2}\right)$ & 0.067 \\
\hline \hline
\end{tabular}

An inverter is used to control and run the $100 \mathrm{~kW}$ permanent magnet (PM100) AC e-motor. The inverter controller is programmed to run the e-motor. Table 4.3 lists the inverter specifications.

\section{Table 4.3}

Inverter specifications.

\begin{tabular}{lc}
\hline \hline Items & Descriptions \\
\hline Maximum DC Voltage continious operating $(\mathrm{V})$ & 360 \\
Maximum DC Voltage non-continous operating $(\mathrm{V})$ & 500 \\
Peak current limit (Arms) & 350 \\
DC bus capacitor $(\mu \mathrm{F})$ & 440 \\
Mass $(\mathrm{kg})$ & 7.5 \\
Coolant & water-glycol \\
\hline \hline
\end{tabular}




\subsubsection{E-motor Torque Tracking Performance}

The driver commanded torque is carried out through a desired torque-based control strategy embedded in the inverter. The driver's desired speed setpoint is controlled by the dyno controller. The inverter controls the e-motor to track the reference torque, which is determined by the operator through the dSPACE ControlDesk ${ }^{\circledR}$ interface. Furthermore, the setup is capable of implementing the regenerative braking test scenarios to capture the braking energy and charges the battery. The regenerative braking happens when the torque command is negative or the motor direction is reversed. Figure 4.5 shows the e-motor traction test scenario for a dyno constant speed. The e-motor is tracking the commanded torque (Fig. 4.5-a), which is sent and control through the supervisory controller. This type of experiments are used to develop the electric powertrain efficiency maps for different testing and load conditions. The powertrain efficiency maps are then used in the mode-based supervisory control strategy implementation.

As Figure 4.5] shows the SOC drops from 40 to 30 percent (Fig. 4.5-f) while the motor torque reaches to 110 N.m at 1000 rpm constant speed. The battery and motor cooling systems operate continuously to keep the temperature within the desired threshold (Fig. 4.5-e). Moreover, the combined loss and efficiency data of the powertrain -from the battery to the dyno shaft- can be obtained from Figure 4.5-g. 
(a) Torque Tracking Performance

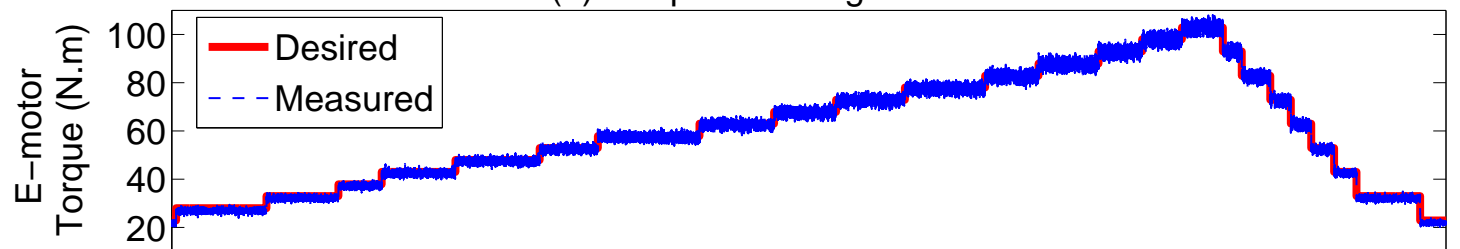

(b) Motor Speed

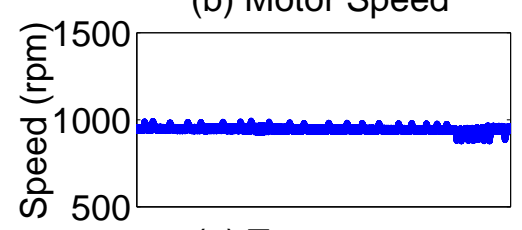

(e) Temperature

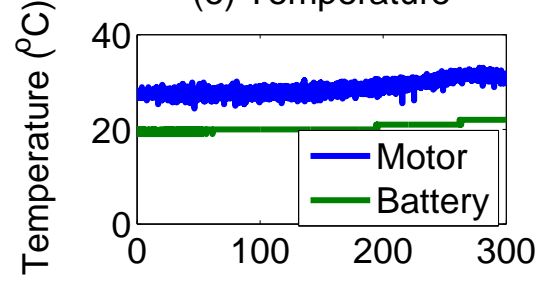

(c) Currents

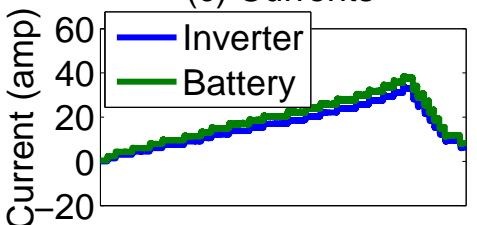

(f) SOC

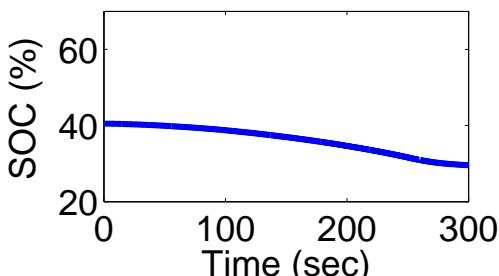

(d) Voltages

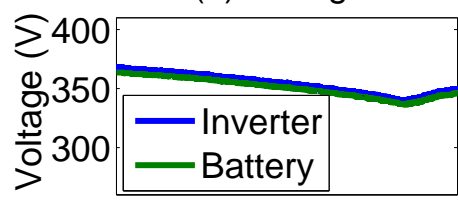

(g) Power

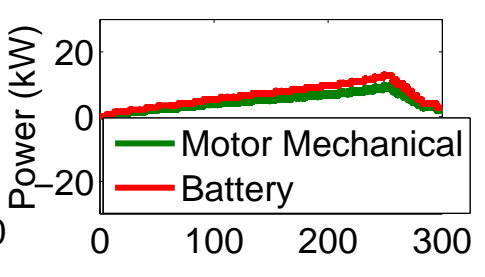

Figure 4.5: E-motor traction test results.

\subsubsection{E-motor and Battery Characteristics Maps}

Figure 4.6 illustrates the LG Chem battery charging and discharging power limits versus SOC. The LG Chem battery SOC operating window is from 70 to 30 percent. As expected, the battery charging power is smaller when the battery is fully charged. However, the battery has a higher discharging power limit at high SOC. This data will be used in the powertrain modeling for the design of the supervisory energy management strategy.

Figure 4.7 shows the e-motor efficiency map during different e-motor speeds and torques. The data is collected at the e-motor temperature of $45^{\circ} \mathrm{C}$ and the DC 


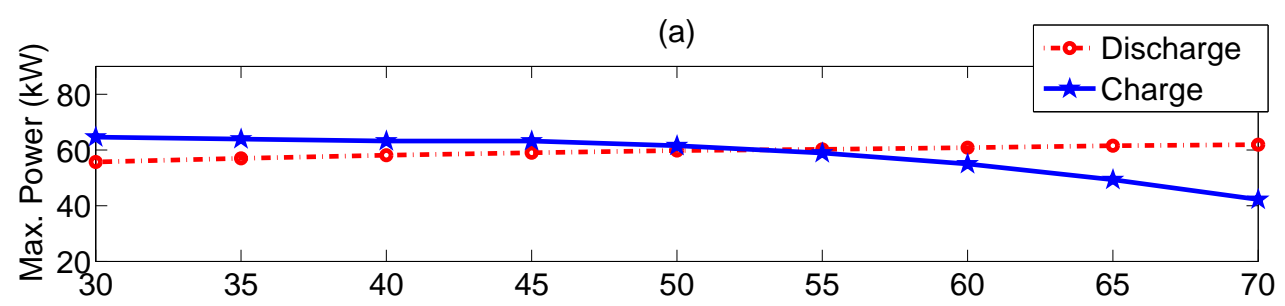

(b)

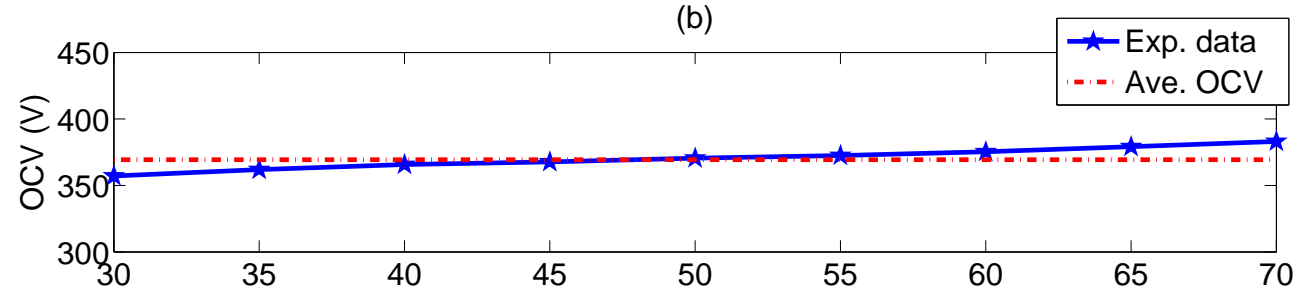

(c)

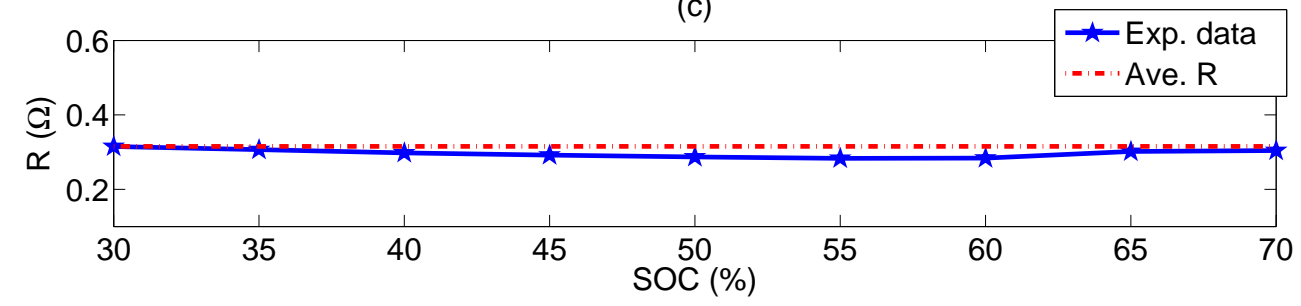

Figure 4.6: High voltage battery specifications: a) charge and discharge power limit, b) open circuit voltage (OCV), and c) internal resistance variation vs. SOC at battery temperature of $25^{\circ} \mathrm{C}$.

voltage of $360 \mathrm{~V}$. Under these conditions, the e-motor efficiency ranges from 71.7 to 91.2 percent.

In next section the engine setup is introduced and the necessary engine map-based models for this study are presented. 


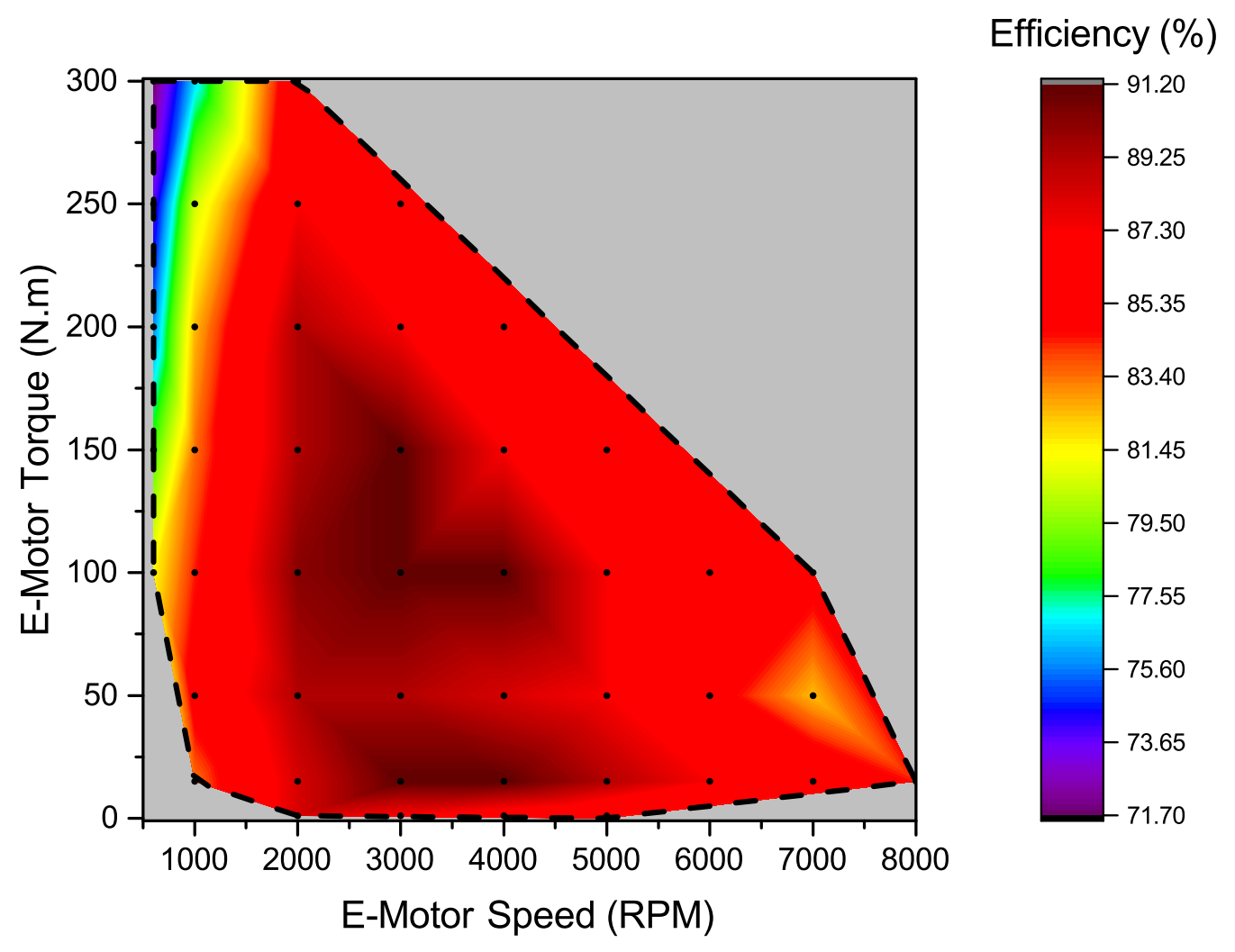

Figure 4.7: Efficiency map of the $100 \mathrm{~kW}$ synchronous PMSM Remy motor including the transmission efficiency. Test condition: e-motor temperature $=45^{\circ} \mathrm{C}$ and DC bus voltage $=360 \mathrm{~V}$.

\subsection{Multi-Mode Engine Setup}

The baseline engine setup includes a GM 2.0-liter Ecotec Gasoline Direct Injection Turbocharged SI engine. Table 4.4 lists the engine specifications. The baseline SI engine was converted to a multi-mode LTC-SI engine as shown in Figure 4.8. LTC modes include HCCI and RCCI operations. Major changes to the baseline SI engine include (i) design of control strategies and programming new engine control unit 
(ECU), (ii) adding port fuel injection systems, and (iii) capability to adjust intake charge temperature, pressure, and dilution level through utilizing intake air heater, supercharger, and exhaust gas recirculation (EGR) rate modulation, respectively.

Table 4.4

Parameters of the baseline engine in this study

\begin{tabular}{|l|c|}
\hline \hline Parameters & Value/Description \\
\hline \hline Engine Model & GM Ecotec LHU \\
Bore x Stroke & $86 \times 86 \mathrm{~mm}$ \\
Number of Cylinders & 4 \\
Displacement Volume & $2.0 \mathrm{~L}$ \\
Compression Ratio & $9.2: 1$ \\
Connecting Rod Length & $145.5 \mathrm{~mm}$ \\
Max Power & 270 hp @6000 rpm \\
Fuel Injection System & Gasoline Direct Injection \\
Valve System & DOHC 4 Valves \\
\hline
\end{tabular}

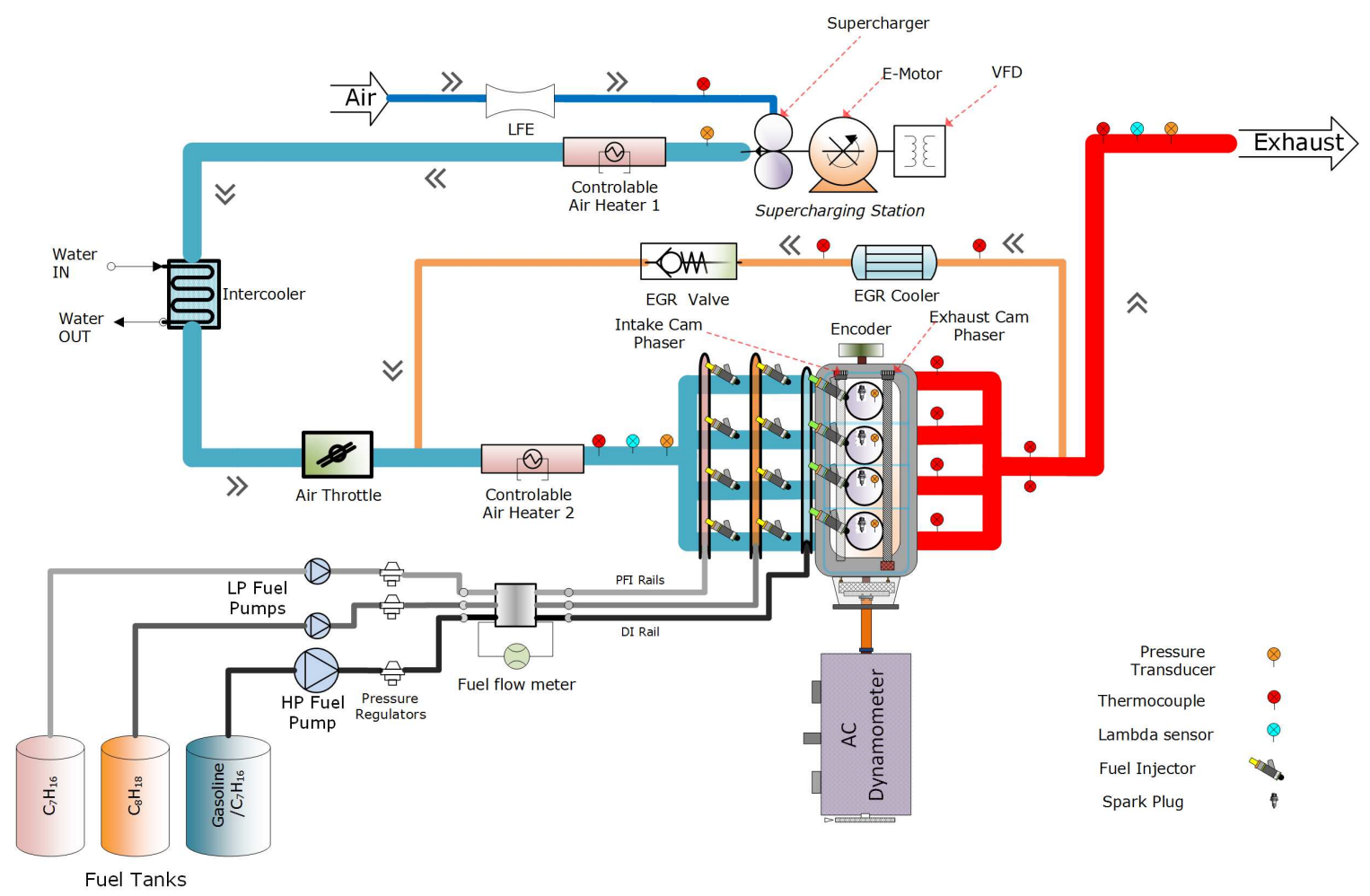

Figure 4.8: Schematic of fuel-flexible multi-mode LTC-SI engine test setup. 
Engine control units included dSPACE ${ }^{\circledR}$ MicroAutoBox (MABx) DS1511 and RapidPro units. Models for control of cam phasers, fuel pump, injectors, spark plugs, supercharger, throttle body, and EGR valve were developed in Simulink ${ }^{\circledR}$. These models were compiled into a single engine control program, and related parameters were monitored and controlled in real-time using the dSPACE ControlDesk ${ }^{\circledR}$. The $\mathrm{ACAP}^{\circledR}$ system was used as the combustion analyzer and the in-cylinder pressures were measured using PCB 115A04 piezo electric pressure transducers. A signal amplifier was used to send the measured signals to ACAP for post-processing. The crank angle was measured with 1 crank angle degree resolution. National Instruments PXIe 1078 chassis system, which included NI PXI 6722, NI TB 4353, and NI PXI 6225 modules, was used for dynamometer control, thermocouple measurement, and pressure transducer measurement, respectively.

Two fuel rails were installed at the interface of the intake manifold and cylinders for port fuel injection. A low-pressure external fuel pump was used to supply fuel at 3 bar pressure to the port fuel injectors and a high-pressure fuel pump was used to supply fuel at 100 bar pressure to direct fuel injectors. The injection system has the capability of supplying three fuels to the engine at the same time. The amount of fuel injected was controlled using $\mathrm{dSPACE}^{\circledR}$ MABx. Pressures (coolant, oil, and intake air) and temperatures (exhaust gas, intake air, coolant and oil gallery) were measured using piezo resistive pressure transducers with an accuracy of $\pm 0.5 \%$ and K-type thermocouples with an accuracy of $\pm 0.75 \%$, respectively. The intake air was 
preheated using two air heaters between the intake manifold and the supercharging station. The mass flow rate of intake air was measured using Merriam MDT500 air flow measurement system. The engine speed and load were controlled using a $465 \mathrm{hp}$ General Electric AC dynamometer.

Experiments were performed for three different combustion modes, namely SI, HCCI, and RCCI. Operating conditions such as intake air temperature $\left(T_{\text {intake }}\right)$, intake manifold pressure $\left(P_{\text {intake }}\right)$, research octane number $(\mathrm{RON})$ of fuel, engine speed $(N)$, and equivalence ratio $(\phi)$ were varied individually keeping the other parameters constant. The operating conditions used for each of the combustion modes are given in Table 4.5. The experiments were conducted for a range of engine speeds and a range of equivalence ratios between the knock and misfire limits. Thereby, the operating region and load limit for each combination of input parameters were determined.

Table 4.5

Engine test setting for SI and LTC operating modes

\begin{tabular}{|l|c|c|c|}
\hline \hline Combustion Mode & SI & HCCI & RCCI \\
\hline \hline Fuel Injection System & DI & PFI1+PFI2 & DI+PFI1 \\
Fuel Type & Gasoline/iso-octane & n-heptane+ iso-octane & n-heptane + iso-octane \\
Engine Speed (rpm) & $800-4000$ & $800-1600$ & $800-2200$ \\
SOI $(\mathrm{CAD}$ bTDC) & 100 & 450 & Variable \\
IVO $(\mathrm{CAD}$ bTDC) & -24.5 & 25.5 & 25.5 \\
EVC $(\mathrm{CAD}$ bTDC) & 22 & 22 & 22 \\
$T_{\text {intake }}\left({ }^{\circ} \mathrm{C}\right)$ & 40 & $40,60,80,100$ & $40,40,80,100$ \\
$P_{\text {intake }}(k P a)$ & 100 & 100 & 100 \\
RON $(-)$ & 87 & $0-40$ & $20-60$ \\
\hline
\end{tabular}




\subsubsection{Engine Experimental BSFC and Temperature Maps}

Using the data acquired from $\mathrm{dSPACE}^{\circledR}$, LabVIEW $^{\circledR}$ and $\mathrm{ACAP}^{\circledR}$, the combustion and performance parameters were calculated using an in-house Matlab ${ }^{\circledast}$ code. The brake specific fuel consumption (BSFC) maps were generated and the load limits for each of the combustion modes were determined. Figure 4.9 shows the BSFC maps for SI, HCCI and RCCI combustion modes with engine speed (RPM) on the x-axis and brake torque (N.m) on the y-axis. 


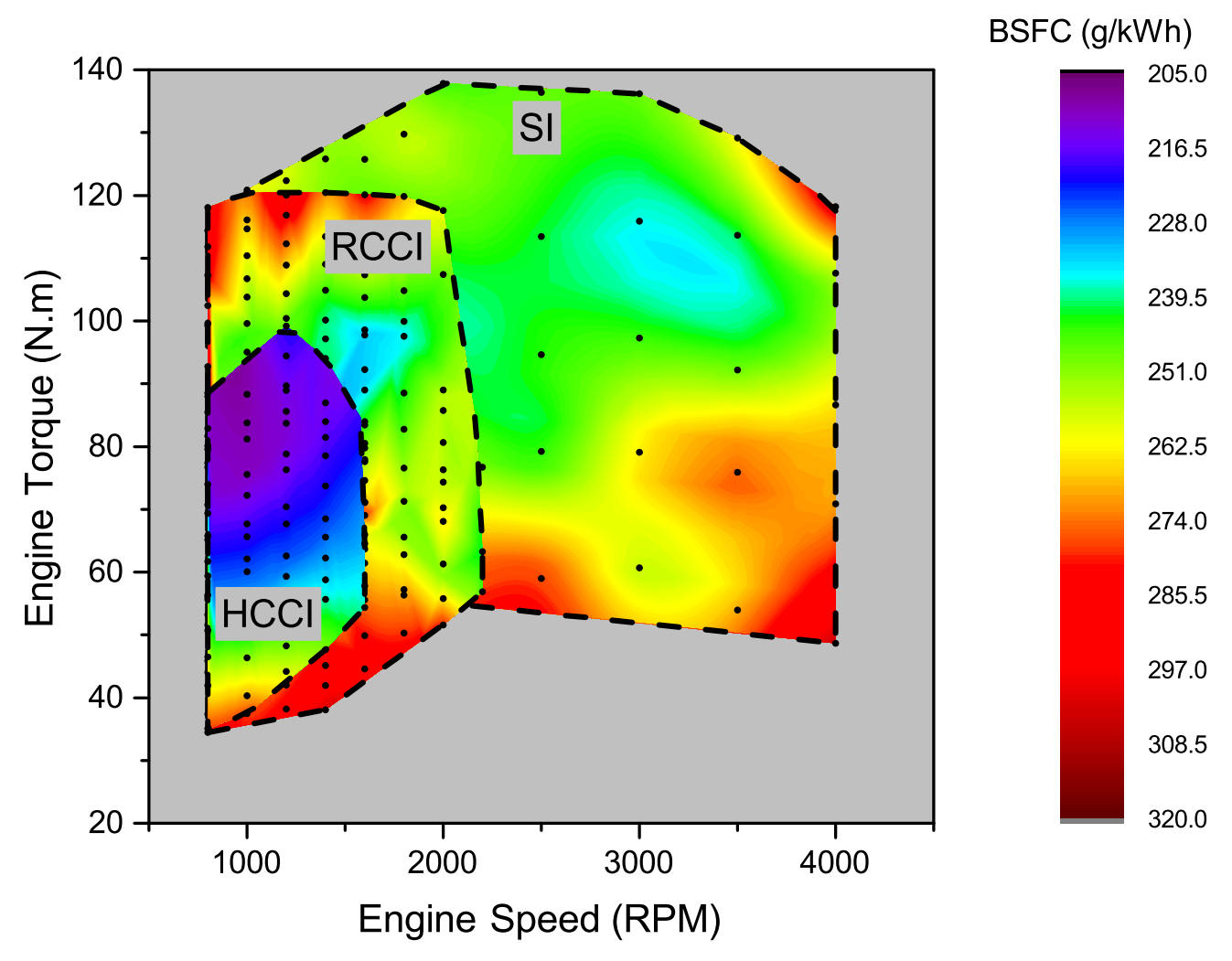

Figure 4.9: Experimental BSFC map of the developed multi-mode LTC-SI engine. Data points are shown by dot symbols.

The measured exhaust gas temperatures are shown in Figure 4.10 for SI, RCCI, and HCCI operating modes. The SI engine exhaust gas temperature ranges from $457^{\circ} \mathrm{C}$ to $776{ }^{\circ} \mathrm{C}$. This temperature range changes to $246-660^{\circ} \mathrm{C}$, and $200-442^{\circ} \mathrm{C}$ for the RCCI and HCCI operating modes, respectively. Given $\mathrm{CO}$ and $\mathrm{HC}$ conversion efficiency in the exhaust catalytic converter is a function of the catalyst temperature, the engine operating points are selected to meet the minimum catalyst light-off temperature (i.e., $300{ }^{\circ} \mathrm{C}[89]$ ). This is realized by considering the engine exhaust gas temperature as a 
constraint in the optimization framework, which will be discussed in Chapter 6 .

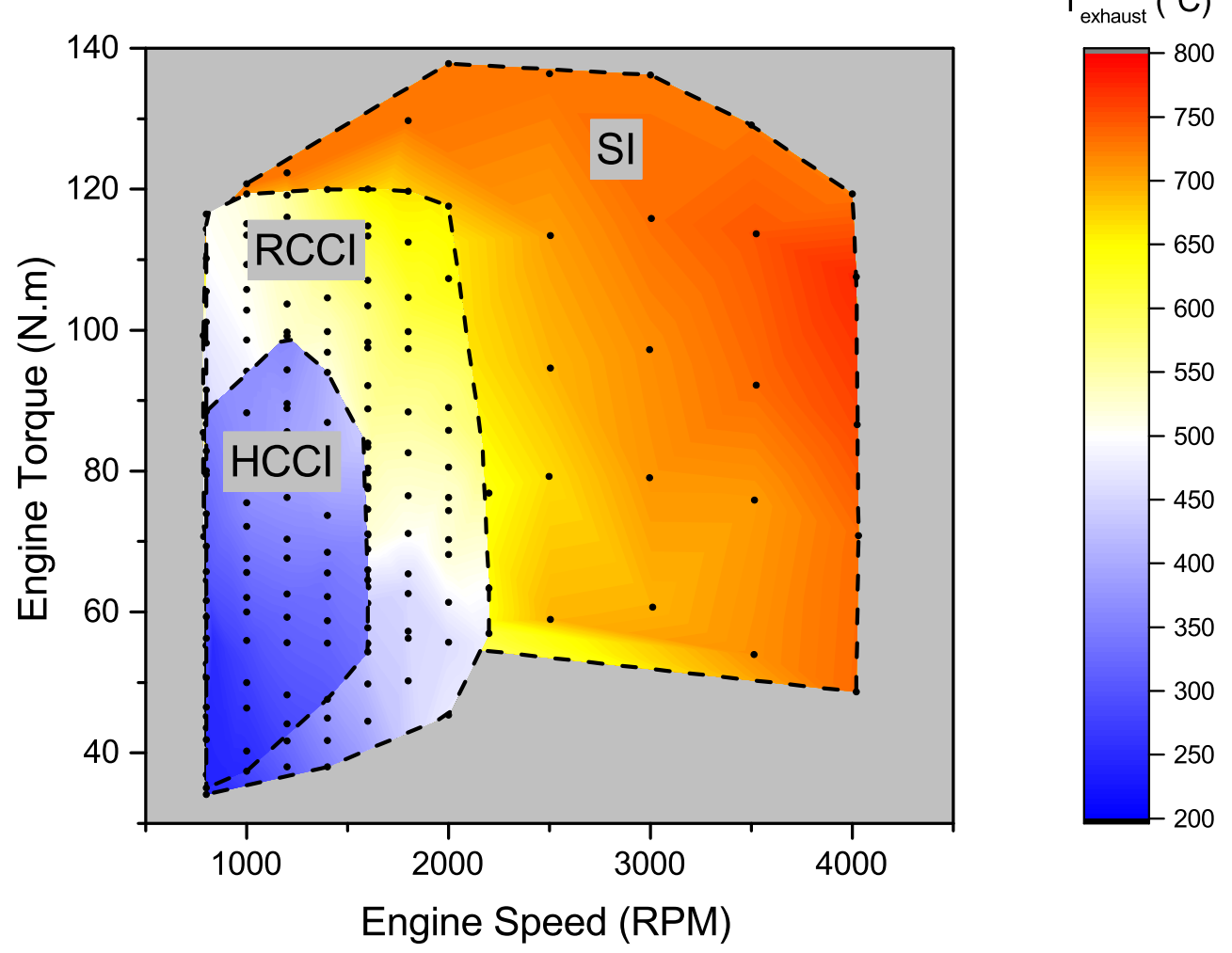

Figure 4.10: Experimental temperature map of the developed multi-mode LTC-SI engine. Data points are shown by dot symbols.

\subsubsection{Experimental Mode-Switching Fuel Penalty Map}

For the multi-mode engine, it was necessary to develop mode-switching strategies to utilize the engine at its best operating mode including HCCI, RCCI or SI mode. These strategies were developed by Jayant Arora [9]. This allows the engine to utilize the high thermal efficiency of LTC modes at lower to mid loads and also full load 
range operation of SI mode. To this end, mode switching strategies among SI and LTC modes were developed. A switch to SI-mode is needed at the point where either SI mode is more efficient compared to LTC modes or LTC combustion is not possible. Optimum combustion phasing and load were set for the SI mode by tuning the throttle position, fuel quantity and spark timing. Similarly, for RCCI mode, start of injection (SOI) and premixed ratio (PR) were adjusted. The tuned steady state points for each mode were fed as a feedforward command. As soon as the switch was activated, the actuators including throttle, fuel injectors and cam phasors, were varied to attain the required set-point.

For achieving SI to RCCI switch without any misfires, spark assist was provided for 2-3 engine cycles after the switch was activated. This aids auto-ignition of the airfuel mixture in the RCCI mode. For RCCI to SI switch, to avoid the mixture from becoming too lean while the air condition stabilizes, a strategy for injecting extra fuel was devised. When switching to SI mode, all the fuel came from PFI rail. To compensate for the delay in PFI fueling and air stabilization, direct injection fueling was kept active for 1 cycle after the switch.

A similar strategy was taken for HCCI-SI switching. In this case, SI was done utilizing DI rail where as PFI rails were utilized for HCCI. Owing to this, the strategy was reversed; that was, for SI to HCCI switching extra fuel was injected. For HCCI to SI, the spark supported switch was carried out, which resulted in high maximum pressure 
rise rate (MPRR). Results for both the switches are shown in Figures 4.11 and 4.12 , 
(a)

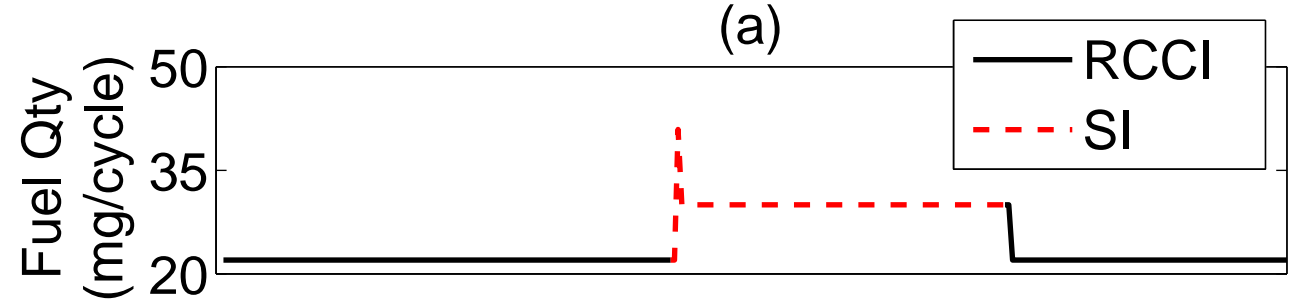

(b)

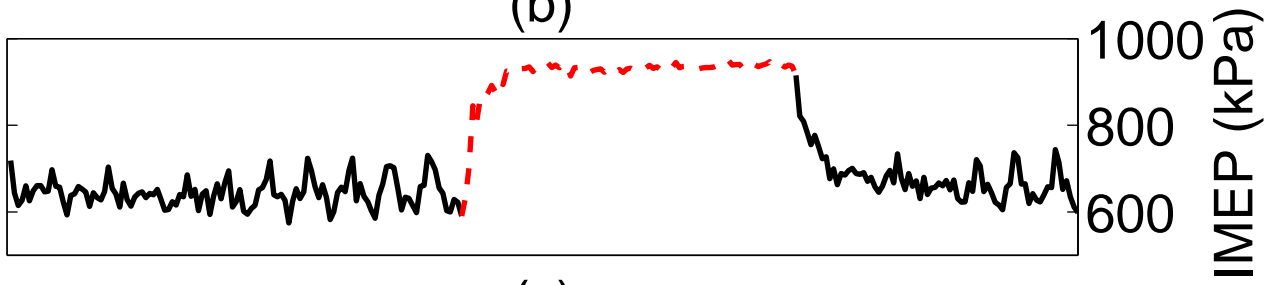

(c)
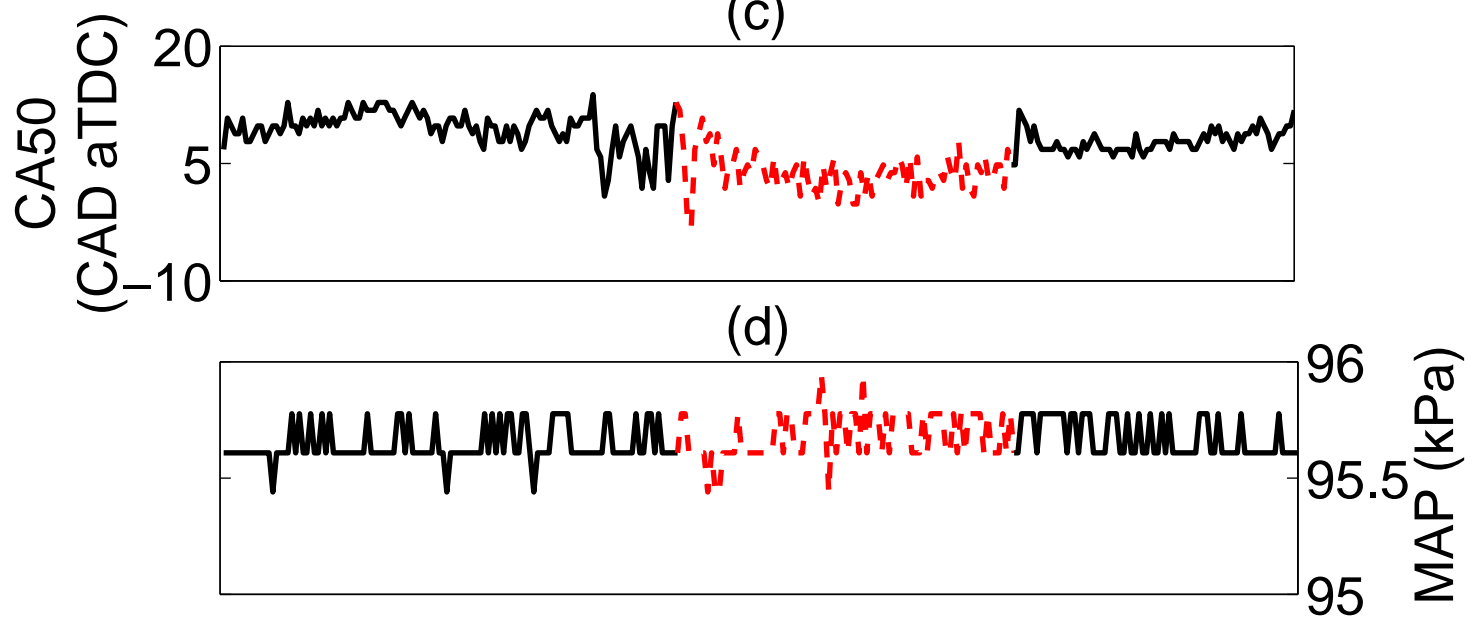

(e)

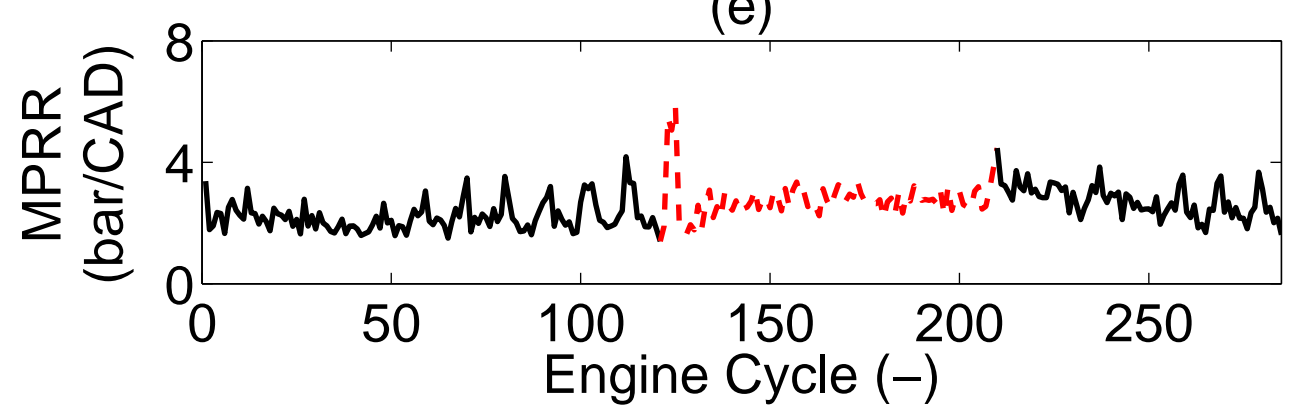

Figure 4.11: RCCI to SI mode transient experiment, where IMEP and MAP stand for indicated mean effective pressure and manifold absolute pressure, respectively. Engine speed $=1300 \mathrm{RPM}, T_{\text {intake }}=60^{\circ} \mathrm{C}$ and remaining test conditions are the same as those in Table 4.5. The data in this figure was collected as part of the study in [9] by Jayant Arora. Permission letter is included in Appendix D. 
(a)
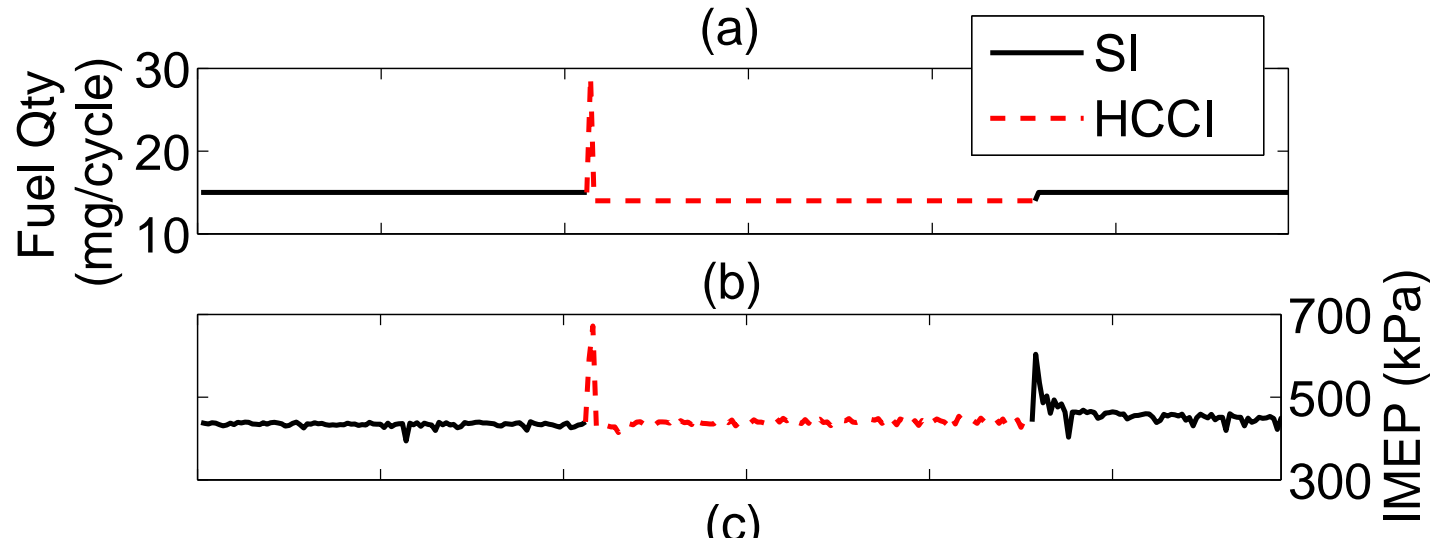

(c)
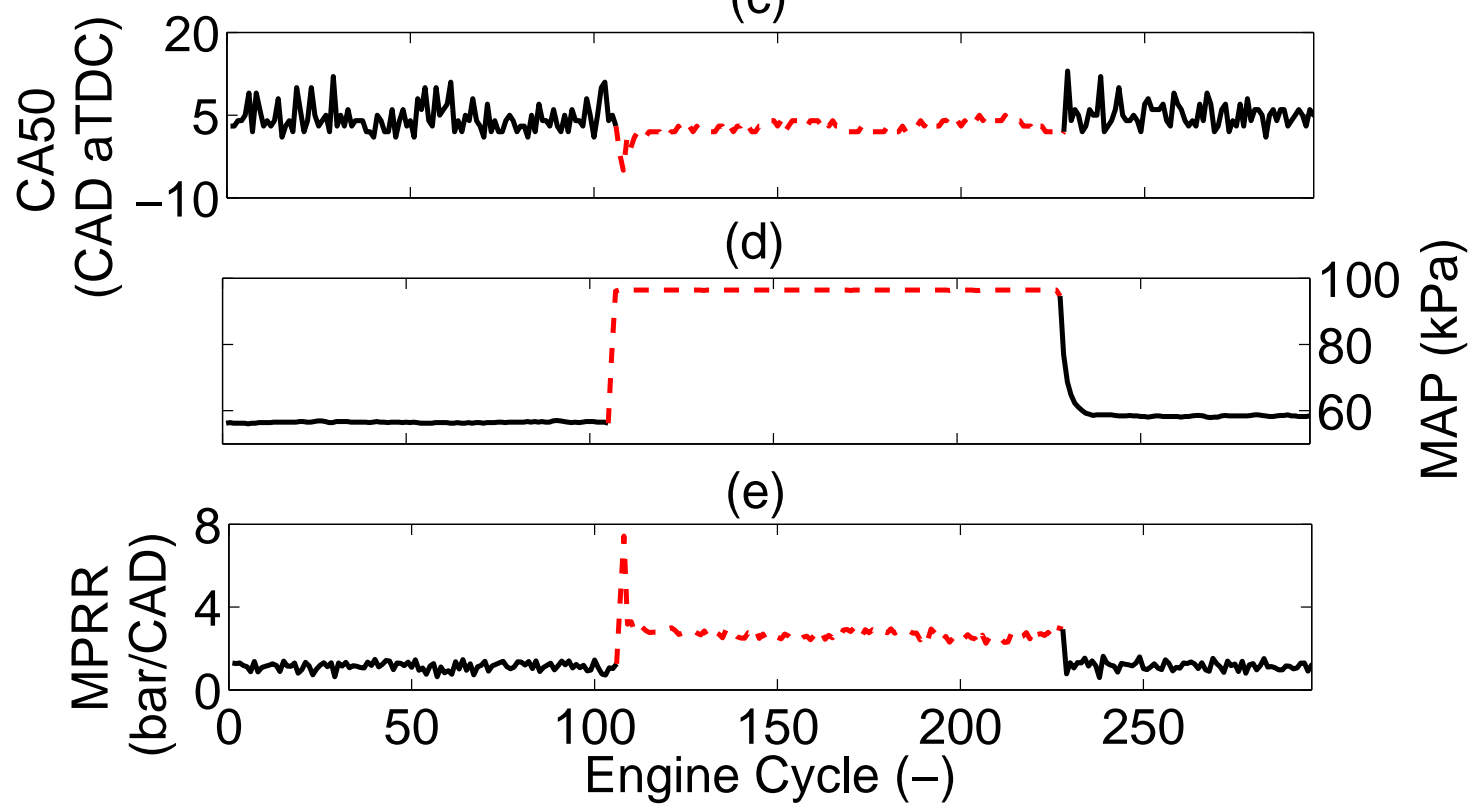

Figure 4.12: HCCI to SI mode transient experiment. Engine speed = $1200 \mathrm{RPM}, T_{\text {intake }}=60{ }^{\circ} \mathrm{C}$ and remaining test conditions are the same as those in Table 4.5. The data in this figure was collected as part of the study in [9] by Jayant Arora. Permission letter is included in Appendix D. 


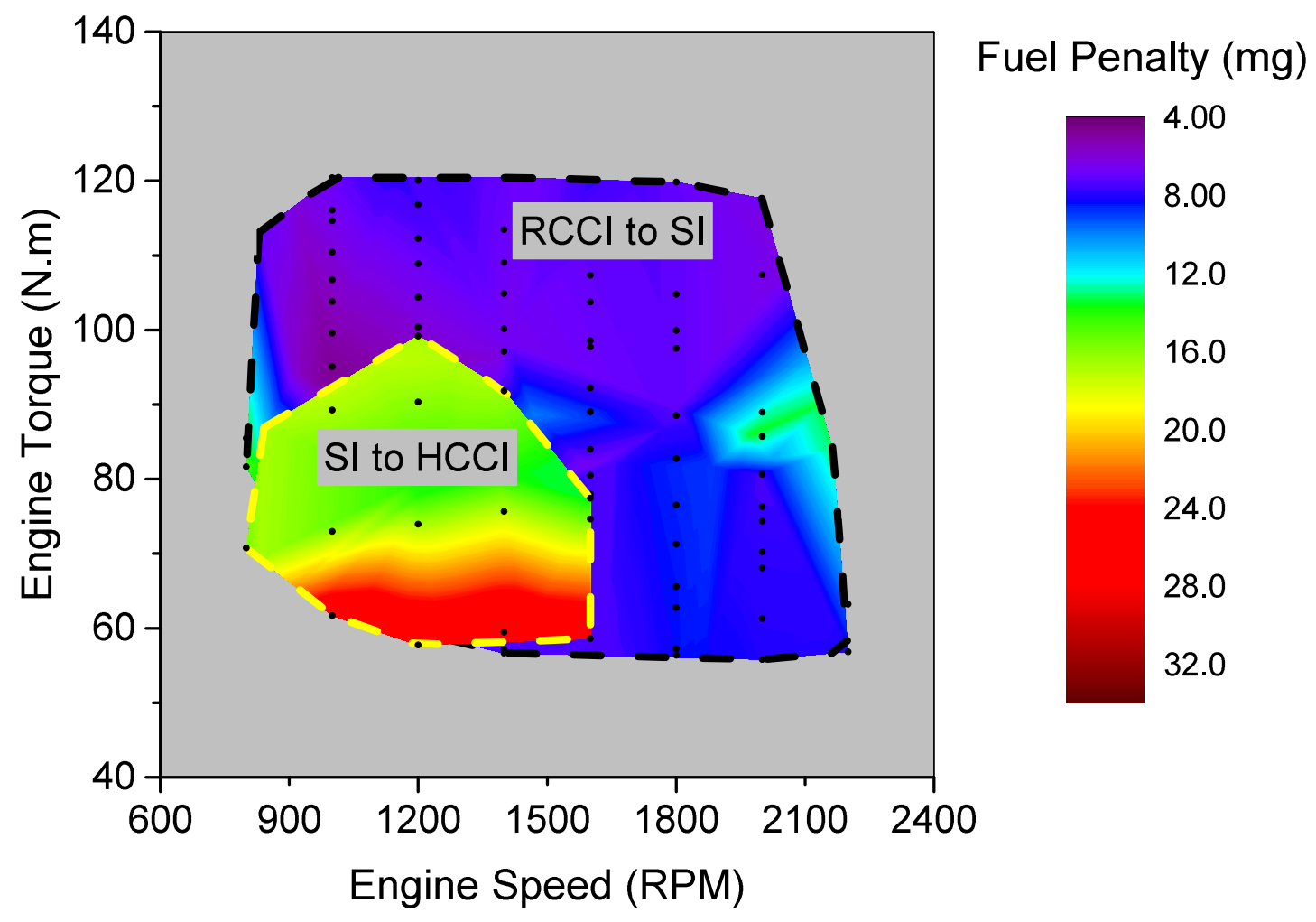

Figure 4.13: Experimental mode-switching fuel penalty map.

Figure 4.13 highlights the fuel penalty map for switching from SI to HCCI and RCCI to SI for different engine speeds and torques. This map will be later used in Chapters 5. and 6] as part of the cost function in the optimization framework to minimize powertrain energy consumption. 


\section{Chapter 5}

\section{Multi-Mode LTC-SI Range Extender 1}

Chapters 2 and 3 investigated hybridizing "single-mode" LTC enignes. Using the experimental data from the test setup in chapter 4 , this chapter investigates fuel economy benefits of hybridizing "multi-mode" LTC engines which allows for maximum engine efficiency. In this chapter, the global optimum fuel economy improvement of the experimentally developed 2-liter multi-mode LTC engine (chapter 4) in a series E-REV is investigated. The engine operation modes include Homogeneous-Charge Compression Ignition (HCCI), Reactivity Controlled Compression Ignition (RCCI),

\footnotetext{
${ }^{1}$ The materials in this chapter are partially based on the pre-submition journal paper in [4] with permission from Elsevier as shown in Appendix D
} 
and conventional Spark Ignition (SI). The simulation results show in the city driving cycle, the single-mode HCCI and RCCI engines offer $12 \%$ and $9 \%$ fuel economy improvement, respectively over a single-mode SI engine in the E-REV. These improvements increase to $13.1 \%$ and $10.3 \%$ in the highway driving cycles. In addition, the mode-switching fuel penalty is included in the optimization problem and the results are used to determine number of LTC modes. The results show that the multi-mode LTC engine offers $2 \%$ more fuel economy improvement over the best single-mode LTC engine operation. These results depend on the type of driving cycle and modeswitching fuel penalty. HCCI and RCCI engine modes can be the dominant optimal engine operating modes depending on the mode-switching fuel penalty value.

\section{$5.1 \quad$ Introduction}

Figure 5.1 shows the updated summary of prior studies in the literature. For brevity, only new parts are explained here, as other parts were previously explained in chapters 2 and 3 .

In majority of the previous works, the engine has been flexible to switch from a singlemode LTC to a conventional mode [15, 34, 35, 36], while this chapter is the first study of integrating a multi-mode LTC engine in HEVs. Thus, the engine not only could switch to conventional modes, but also it could switch among different LTC modes. 


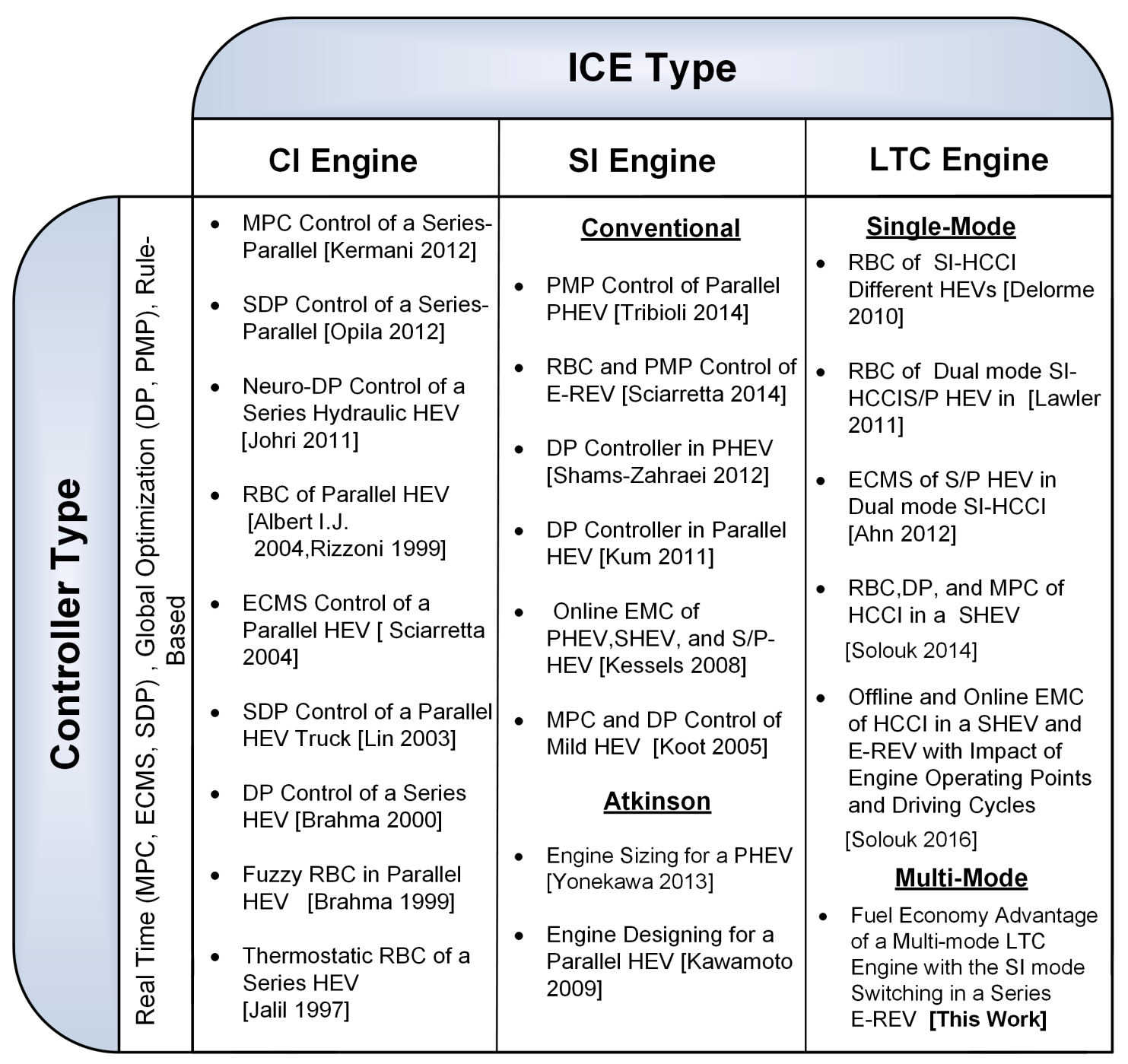

Figure 5.1: Different types of ICEs and control techniques used in HEVs in previous studies.

To the best of the author's knowledge, this is the first study undertaken to investigate the fuel economy benefit of integrating multi-mode LTC engine with series E-REV equipped with an advanced optimal control strategy. The contribution from this chapter is fourfold. First, it investigates the ultimate fuel saving of dedicated different 
LTC-modes in series E-REV configuration. Second, it investigates the effect of multimode LTC fuel saving over the single-mode LTC in the series E-REV. Third, it studies the trade-off between the number of engine modes and the engine modeswitching fuel penalty on the powertrain fuel economy. Lastly, it introduces a criteria for performance of the engine mode-switching controller in order to utilize a multimode LTC engine in a series E-REV.

This chapter is organized as follows. The first section investigates the optimal control problem in the series E-REV architecture and applies the PMP optimization techniques. Then, the results of the series E-REV for the single-mode and multi-mode engine operations are discussed. Finally, the last section summarizes all findings from this chapter.

\subsection{Design of Optimal Control for Multi-Mode LTC-Based E-REV}

The goal of the optimal control in this chapter is to minimize the fuel consumption $\left(\dot{m}_{f}\right)$ using the cost function defined by Eq. (5.1):

$$
J(u(t))=\int_{0}^{T} \dot{m}_{f}(t) d t
$$


where $\dot{m}_{f}$ is the rate of the engine fuel consumption and $T$ is the time length of a driving cycle. Equation (2) shows the constraints for the HEV optimization problem.

$$
\begin{aligned}
& \left|S O C_{f}-S O C_{0}\right| \leqslant \epsilon \\
& S O C_{\text {min }} \leqslant S O C(t) \leqslant S O C_{\text {max }} \\
& P_{\text {bat,min }} \leqslant P_{\text {bat }}(t) \leqslant P_{\text {bat,max }} \\
& P_{\text {eng,min }}\left(\omega_{\text {eng }}\right) \leqslant P_{\text {eng }}\left(t, \omega_{\text {eng }}\right) \leqslant P_{\text {eng,max }}\left(\omega_{\text {eng }}\right) \\
& \omega_{\text {eng,min }} \leqslant \omega_{\text {eng }}(t) \leqslant \omega_{\text {eng,max }} \\
& P_{\text {motor,min }} \leqslant P_{\text {motor }}(t) \leqslant P_{\text {motor,max }} \\
& \omega_{\text {motor,min }} \leqslant \omega_{\text {motor }}(t) \leqslant \omega_{\text {motor,max }}
\end{aligned}
$$

The constraints in Eq. (2) are applied for the battery SOC operation window, battery power $\left(P_{b a t}\right)$, engine power $\left(P_{\text {eng }}\right)$, engine speed $\left(\omega_{\text {eng }}\right)$, motor power $\left(P_{\text {motor }}\right)$, and motor speed $\left(\omega_{\text {motor }}\right)$. This optimal control problem is solved using optimal control techniques that are described in next subsection. 


\subsubsection{Pontryagin's Minimum Principal (PMP)}

The PMP method is based on a general case of the Euler-Lagrange equation and is originated from the Calculus of Variation. This method yields the necessary -not sufficient- conditions of the global optimal solution. The optimal trajectories derived from PMP will be the global optimal solution of the HEV problem if the obtained optimal trajectory is a unique trajectory that meets the necessary and boundary conditions [90]. The necessary condition for the PMP global optimality is explained

in [90] and will be briefly explained in the Subsection B. In the next Subsection A, the static model of the target vehicle is described. Moreover, a technique is introduced to determine the Hamiltonian values from the model.

\section{A. Control Variables:}

In the series E-REV architecture in this chapter, the engine is decoupled from the drivetrain, which allows to precisely select the engine operating points. This enables the LTC engine to operate in a narrow engine operating range and satisfies the driving cycle's average power demand. Figure 5.2 shows the series E-REV architecture and Table 5.1 lists the vehicle parameters used in this chapter. The static models and maps of the vehicle components are obtained from the experimental setup explained in chapter 4, 


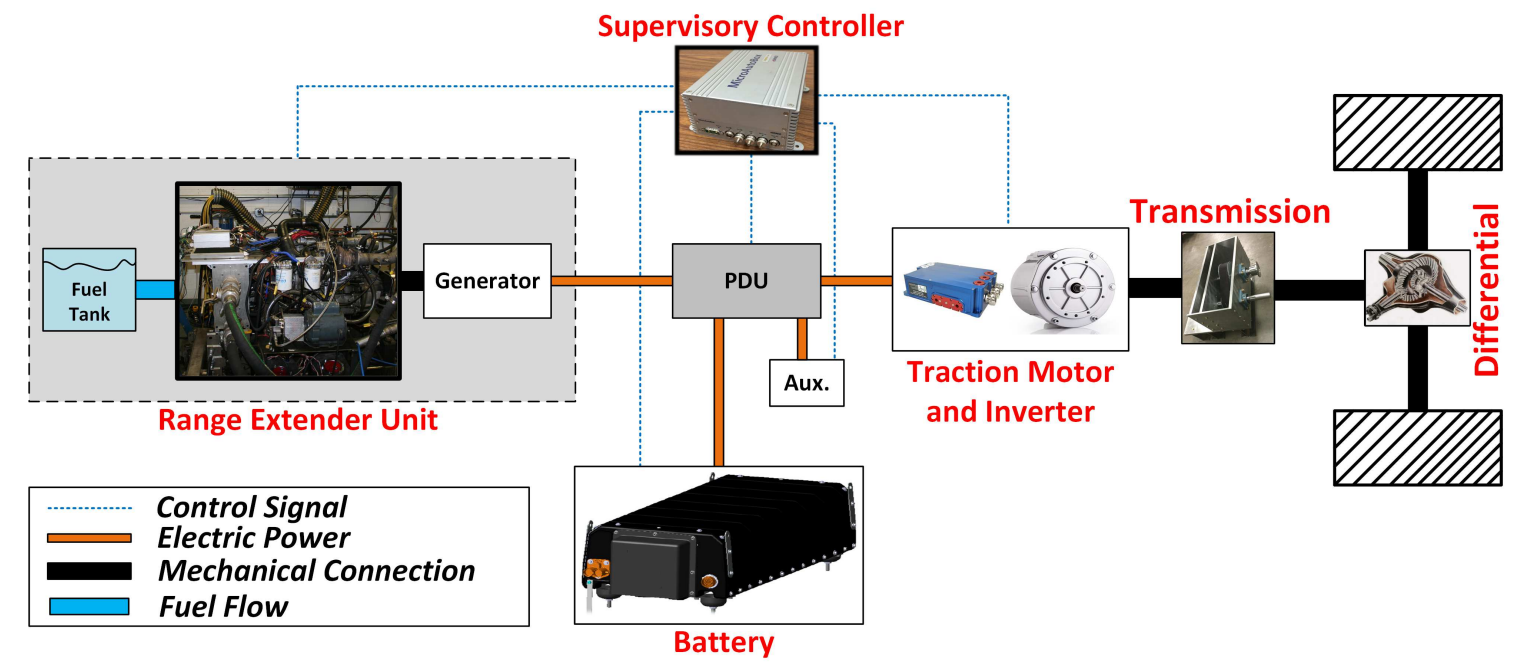

Figure 5.2: Series E-REV architecture in this chapter. PDU denotes power distribution unit.

\section{Table 5.1}

Vehicle specifications.

\begin{tabular}{|l|c|}
\hline \hline Parameters & Values \\
\hline \hline Vehicle Curb Weight $(\mathrm{kg})$ & 1750 \\
Frontal Area $\left(\mathrm{m}^{2}\right)$ & 2.0 \\
Motor Gear Ratio & 2.0 \\
Wheel Radius $(\mathrm{m})$ & 0.432 \\
Drag Coefficient & 0.25 \\
Rolling Resistance Coefficient & 0.01 \\
\hline
\end{tabular}

In the static model of the series E-REV there are two independent control variables.

These variables include the power of the engine and the speed of the engine for a given driver's power request and vehicle speed. The e-motor power (Eq. [5.3) is a function of the driver's requested power that is calculated from the driving cycle. 


$$
P_{\text {motor }, e}= \begin{cases}\frac{P_{\text {wheel }}}{\eta_{\text {motor }} \cdot \eta_{\text {gear }}} & \text { (a) } P_{\text {wheel }} \geqslant 0 \\ P_{\text {wheel }} \cdot \eta_{\text {gen }} . \eta_{\text {gear }} & \text { (b) } P_{\text {wheel }}<0\end{cases}
$$

Where $P_{\text {motor,e }}$ is the e-motor input (electrical) power and $P_{\text {wheel }}$ is the demanded power at wheels. $\eta_{\text {motor }}$ and $\eta_{\text {gen }}$ are the efficiency of the e-motor in the motoring and regenerative braking modes, respectively. $\eta_{\text {gear }}$ is the final drive ratio efficiency. The wheel power is determined by the Longitudinal Vehicle Dynamic equations, previously described in chapter 2 .

By obtaining the $P_{\text {motor,e }}$, the $P_{\text {engine }}$ is calculated to be a function of the battery power $P_{b a t}$. The generator integrated to the engine shaft is assumed to have a constant efficiency. Thus, by knowing the $P_{\text {engine }}$ the generator power $\left(P_{\text {gen }}\right)$ is determined accordingly.

$$
P_{\text {gen }}=P_{\text {motor }, e}-\left(P_{b a t}-P_{a c c}\right)
$$

Where $P_{a c c}$ is the required power by the accessories. The fuel consumption rate is determined from the engine BSFC map as a function of $P_{\text {engine }}$ and $\omega_{\text {eng }}$ :

$$
\dot{m}_{f}=F\left(P_{\text {engine }}, \omega_{\text {eng }}\right)
$$


Finally, the time derivative of SOC is obtained from the battery dynamic equation as follows

$$
S \dot{O} C(t)=-\frac{1}{Q_{\text {nom }}} \cdot \frac{O C V^{2}-\sqrt{O C V^{2}-4 R P_{b a t}(t)}}{2 R}
$$

where $Q_{n o m}$ is the battery nominal energy capacity. The equivalent open-circuit voltage $(O C V)$ and internal resistance $(R)$ are functions of SOC. Thus, the $S \dot{O C}$ is a function of $P_{b a t}$ and SOC. In conclusion, the $\dot{m}_{f}$ in (5.5) and $S \dot{O} C$ in (5.6) depend on $S O C, P_{e n g}$, and $\omega_{e n g}$, if the demanded driving power $\left(P_{w h e e l}\right)$ and vehicle speed are given.

\section{B. Engine Optimal Operating Speed:}

An inner-loop optimization process is introduced to reduce the number of the control variables to one. This optimization process makes Hamiltonian $(H)$ only a function of $P_{b a t}$. The Hamiltonian is defined as

$$
\begin{array}{r}
H\left(P_{b a t}, S O C, t\right)=-\lambda(t) \cdot g\left(P_{b a t}(t), S O C(t)\right) \\
+\dot{m}_{f}\left(P_{e n g}, \omega_{e n g}, t\right)
\end{array}
$$


where $\lambda$ is called 'costate' in the PMP and $g$ is the state equation, which is the battery dynamic equation (Eq. [5.6). Thus, to calculate the Hamiltonian, first the $\dot{m}_{f}$ and $P_{b a t}$ are determeined using control variables, $P_{\text {eng }}$ and $\omega_{\text {eng }}$ from Equations (5.5) and (5.8).

$$
P_{\text {eng }}=L\left(\omega_{\text {eng }}\right)
$$

Equations (5.5) and (5.8) are obtained by solving the static model (5.3) to (5.4) at each time step. An inner-loop optimization is performed to find the best engine speed $\left(\omega_{\text {eng }}\right)$ at each engine output power level $\left(P_{\text {eng }}\right)$. The inner-loop optimization selects the operating points to minimize the fuel consumption as follows.

$$
\begin{array}{r}
\min _{\omega_{\text {eng }}} \quad \dot{m}_{f}=F\left(P_{\text {eng }}, \omega_{\text {eng }}\right) \\
\text { s.t. } \quad P_{\text {eng }}=L\left(\omega_{\text {eng }}\right)
\end{array}
$$

The optimized $\omega_{e n g}^{*}$ is obtained by selecting minimum fuel consumption on each feasible engine power line. Thus the engine optimal operating point is determined by having the $P_{\text {eng }}$. Figure 5.3 shows the optimal engine speeds on different values of $P_{\text {eng }}$ in different engine modes including HCCI, RCCI, and SI. Moreover, Figure 5.3 shows the engine minimum $\dot{m}_{f}$ for generating different amounts of power.

After the inner-loop optimization, $P_{\text {eng }}$ is the only control variable. From (5.4), the 

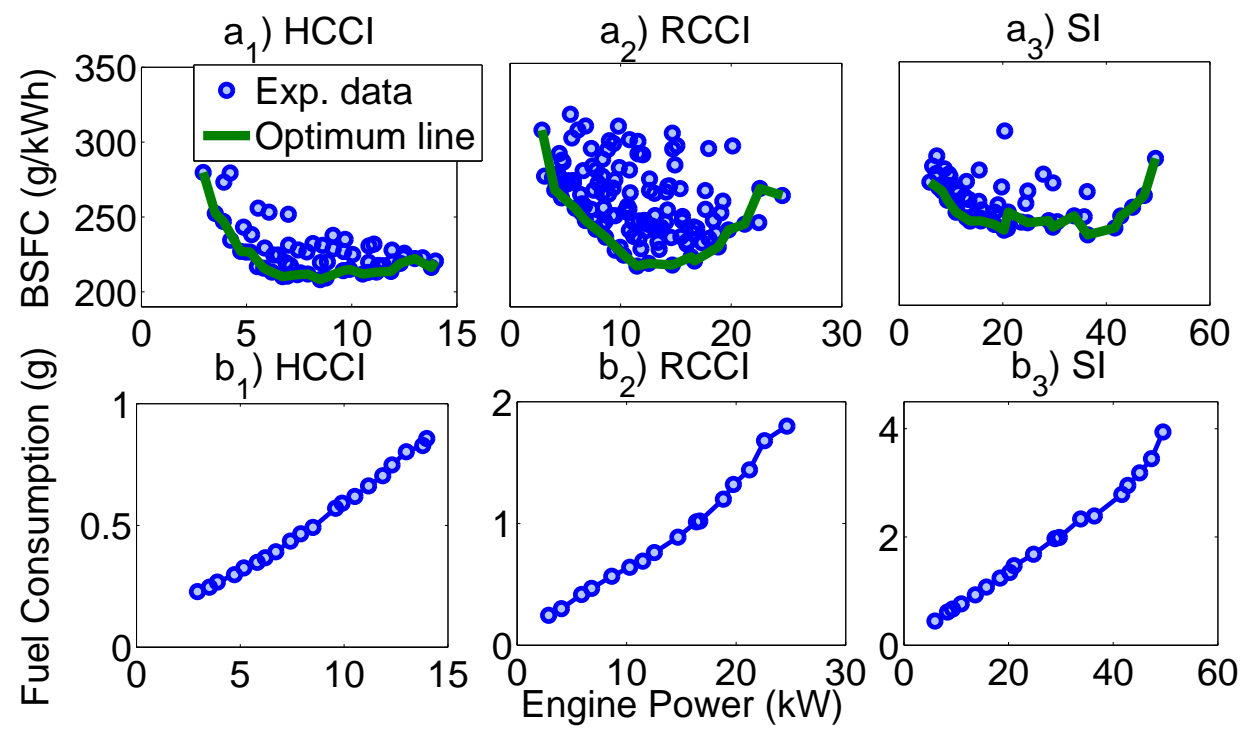

Figure 5.3: a) Finding the best engine speed at each power level based on the minimum BSFC (the green line shows the optimum engine speed); b) the engine lowest fuel consumption at each power level.

$P_{\text {eng }}$ is also a function of $P_{b a t}$ by knowing the power and speed at wheels. Thus, the battery power $\left(P_{b a t}\right)$ is selected as a control variable, which means all other variables can be constant by determining the battery power. This also means the fuel consumption rate can be determined by the battery power $P_{b a t}$ as follows:

$$
\dot{m}_{f}=q\left(P_{b a t}, t\right)
$$

The battery power is shown to be the only control variable that determines all the operating points in the optimal control problem. The battery power determines the engine and generator powers that determine the fuel consumption rate and the optimal speed. The motor speed and power in the series E-REV architecture are variables of 
speed and power at wheels. For obtaining the optimal $P_{b a t}$, the performance index $(J)$ is defined as

$$
\min \left[J=\int_{0}^{T} q\left(P_{b a t}, t\right) d t\right]
$$

In (5.11), $q$ is the fuel rate function from (5.10). Then, Hamiltonian is defined as

$$
H=-\lambda \cdot g\left(P_{b a t}, S O C\right)+q\left(P_{b a t}\right)
$$

Using PMP, the state equation and costate equation are expressed as:

$$
\begin{gathered}
S \dot{O} C=\frac{\partial H}{\partial \lambda} \\
\dot{\lambda}=\frac{\partial H}{\partial S O C}
\end{gathered}
$$

From (5.12) and (5.14), if $g$ is not a function of $S O C$ then the costate can be considered as a constant, which are explained in the following. Figure 4.7 in Chapter 4 illustrated that the $O C V$ and battery resistance $R$ do not vary significantly in the charge-sustaining range of the battery $S O C$ i.e., from 0.3 to 0.7 . In that case, 
the costate stays near the initial value since the $\frac{\partial g}{\partial S O C}$ is negligible compared to the costate for the whole driving cycle. Thus, the costate expression during the SOC usage window is simplified as:

$$
\dot{\lambda}=\lambda \frac{\partial g}{\partial S O C}=0, \quad \rightarrow \lambda=\text { constant }
$$

Obtaining a constant value for the costate reduces the PMP complexity. For optimality the following condition should be considered to specify the optimal control variable $P_{b a t}$ at each time step:

$$
H\left(P_{b a t}^{*}, S O C^{*}, \lambda^{*}, t\right) \leqslant H\left(P_{b a t}, S O C^{*}, \lambda^{*}, t\right)
$$

which means that the optimal control variable $P_{b a t}^{*}$ is the minimum of the Hamiltonian function at the given time. The boundary condition of the final sate variable is:

$$
S O C\left(t_{0}\right)=S O C\left(t_{f}\right)
$$


The $P_{b a t}$, that satisfies equations $(5.13)$ to $(5.16)$ and also the boundary condition (5.17), determines the optimal $P_{b a t}$ trajectory.

If a costate $(\lambda)$ exists that fulfills the condition (5.17), then the PMP provides the 'global optimal' solution [90].

\section{Revising The Cost Function For Multi-Mode Operation:}

The cost function in (5.11) is revised by including the engine startup and modeswitching fuel penalties for minimizing the number of engine starts and modeswitching as

$$
\begin{gathered}
J(u(t))=\int_{0}^{T}\left(\dot{m}_{f}\left(P_{b a t}, t\right)+\Gamma \cdot F_{p}+\Lambda . m_{i j}\right) d t \\
\Gamma= \begin{cases}1 \quad & \text { if } \quad P_{\text {eng }}(t-1) \leqslant \min \left(P_{\text {eng }}(t-1)\right) \\
& \text { and } \quad P_{\text {eng }}(t) \geqslant \min \left(P_{\text {eng }}(t)\right) \\
0 \quad, \text { if } \quad P_{\text {eng }}(t) \leqslant \min \left(P_{\text {eng }}\right)\end{cases}
\end{gathered}
$$

where $\Gamma$ determines the condition for adding the engine startup fuel penalty. $\Lambda$ is zero when the engine is enforced to operate in the single-mode regions and $\Lambda$ is 1 when 
the mode-switching is allowed. The engine startup fuel penalty $F_{p}$ is measured 0.25 grams using our experimental setup.

The $m_{i j}$ is mode-switching fuel penalty to switch from the $i^{\text {th }}$ engine mode to the $j^{\text {th }}$ engine mode. $i \& j \in[1,2,3]$ since the engine can run in HCCI, RCCI, and SI modes. The $m_{i j}$ prevents frequent model-switching between different modes. It accounts for the fuel penalty associated with each mode switching and finds the global optimal solution. The $m_{i j}$ used in this chapter is an experimentally measured value and is taken from 91] for the mode-switching on a similar engine. The mode-switching fuel penalty in [91] is used as a baseline value and the sensitivity of the fuel economy results to the baseline value is investigated in this chapter.

The constraints for the optimal control problem are revised based on the components in the powertrain experimental setup from chapter 4: 


$$
\begin{aligned}
& \left|S O C_{f}-S O C_{0}\right| \leqslant 0.01 \\
& 0.3 \leqslant S O C(t) \leqslant 0.7 \\
& P_{b a t, \text { min }} \leqslant P_{b a t}(t) \leqslant P_{b a t, \text { max }} \\
& P_{\text {eng,min }}\left(\omega_{\text {eng }}\right) \leqslant P_{\text {eng }}\left(t, \omega_{\text {eng }}\right) \leqslant P_{\text {eng,max }}\left(\omega_{\text {eng }}\right) \\
& \omega_{\text {eng,min }} \leqslant \omega_{\text {eng }}(t) \leqslant \omega_{\text {eng,max }} \\
& 0 \leqslant P_{\text {motor }}(t) \leqslant 100 \mathrm{~kW} \\
& 0 \leqslant \omega_{\text {motor }}(t) \leqslant 8000 R P M
\end{aligned}
$$

where a constraint of maximum one percent $\triangle S O C$ variation is considered for the charge-sustaining mode. The next section introduces the results for (i) the singlemode engine and (ii) the multi-mode engine integrated with the series E-REV powertrain. The PMP optimal control solution is used for the analysis in this chapter.

\subsection{Results}

In this section optimization results for the series E-REV-LTC powertrain are discussed. In the first subsection, the results for the dedicated single-mode engines including HCCI, RCCI, and SI in the series E-REV are presented. Then, fuel economy results, and powertrain energy analysis are discussed. In the next subsection, 
the results for integrating a multi-mode LTC engine in the series E-REV are explained. Finally, the ultimate fuel saving for including different engine modes into the powertrain is discussed.

\subsubsection{Single-Mode LTC Engine}

The main goal of charging-path optimization is to make the engine and generator operate at their optimum efficiency points. The operating points of the discharging-

path, on the other hand, are dictated by the road load and vehicle design parameters. The costate $\lambda$ in the PMP is selected such that the SOC is balanced at the end of the driving cycle. For each cycle, depending on available negative power at the wheels, the PMP methodology optimizes the system operating points such that the battery SOC stays as close as possible to the SOC in which the battery has the lowest resistance. In fact, the dependency of battery resistance and voltage on SOC makes the charging-path optimization linked to the driving cycle.

Figure 5.4 shows the engine efficiency maps for the HCCI, RCCI and SI engines. The optimum engine operating points are shown on the maps for the HWFET driving cycle. As can be seen the HCCI engine has a limited operating range in comparison to the SI engine. The HCCI engine maximum power is $14 \mathrm{~kW}$, compared to $25 \mathrm{~kW}$ and $50 \mathrm{~kW}$ for the RCCI and SI engines, respectively for the test conditions in this 
chapter. Further, the HCCI and SI engines are at the two opposite sides of spectrum with highest and lowest available maximum Brake Thermal Efficiency (BTE), i.e., the minimum BSFC.

Tables 5.2 and 5.3 list the fuel consumption, average engine BTE and engine ON time for the UDDS and HWFET cycles. From Tables 5.2 and 5.3 it can be seen that the HCCI engine has the lowest fuel consumption among different engine modes and the SI engine has the highest fuel consumption during both the UDDS and HWFET driving cycles. Due to relative independence of battery charging and discharging paths, the PMP optimization method is successful at keeping all three ICE modes operate close to their highest efficiency areas (see Figure 5.4). Given the generator loss is assumed constant, the amount of engine work for all three ICE modes are very close. Thus, the main difference for hybridization benefits in these three modes comes from the engine efficiency. Hence, the best fuel consumption is achieved with the engine with the highest BTE (i.e., the HCCI engine).

Table 5.2

Series E-REV performance results for using a single-mode engine during the $\underline{\text { UDDS }}$ driving cycle

\begin{tabular}{|l|c|c|c|c|}
\hline \hline $\begin{array}{l}\text { Engine } \\
\text { mode }\end{array}$ & $\begin{array}{c}\text { Fuel consumption } \\
\text { (grams) }\end{array}$ & $\begin{array}{c}\text { Ave. engine } \\
\text { BTE }(\%)\end{array}$ & $\begin{array}{c}\text { Engine ON time } \\
(\mathrm{sec})\end{array}$ & $\begin{array}{c}\text { Engine work } \\
(\mathrm{MJ})\end{array}$ \\
\hline \hline HCCI & 399.8 & 38.1 & 607 & 6.9 \\
RCCI & 415.0 & 38.0 & 483 & 7.0 \\
SI & 448.1 & 34.7 & 228 & 8.2 \\
\hline
\end{tabular}

From Figure 5.4 it can be seen that compared to the HCCI engine, the high BTE 

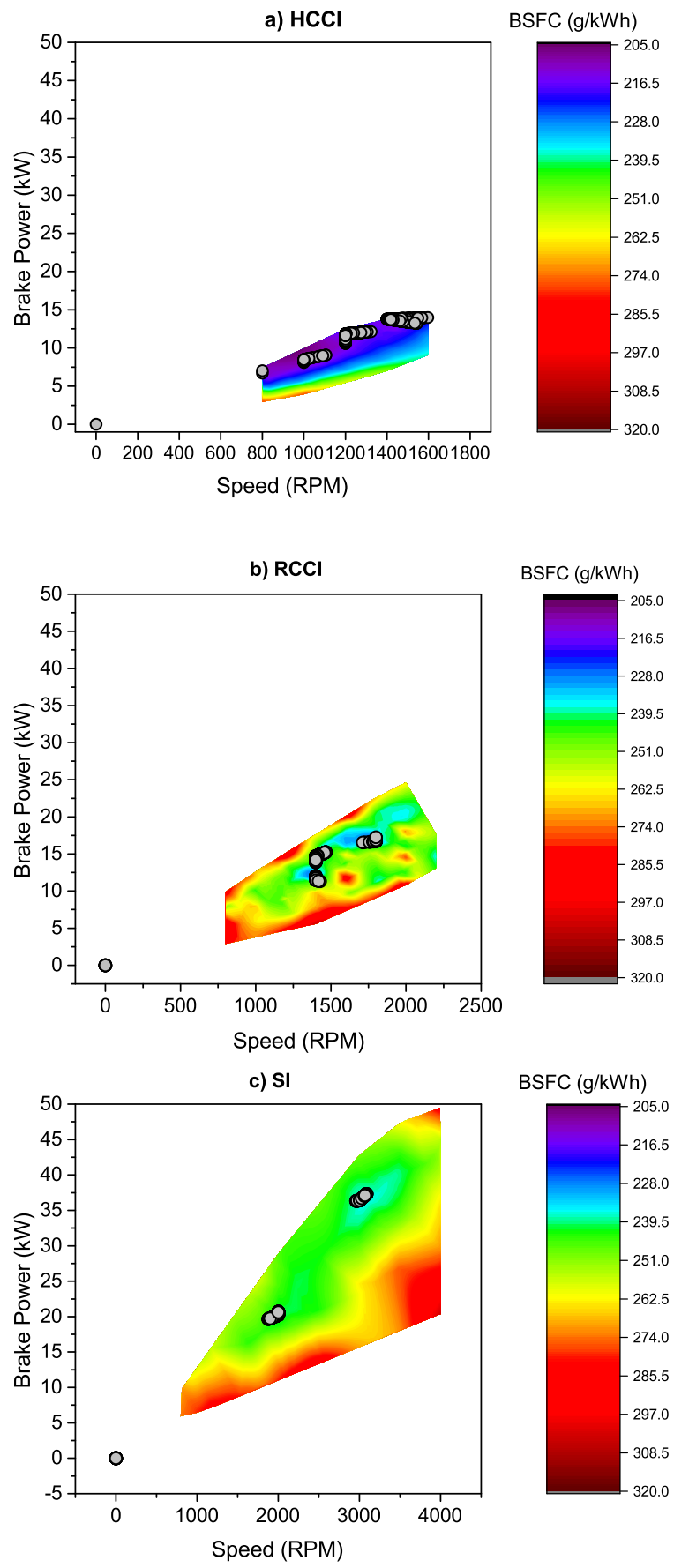

$\mathrm{BSFC}(\mathrm{g} / \mathrm{kWh})$

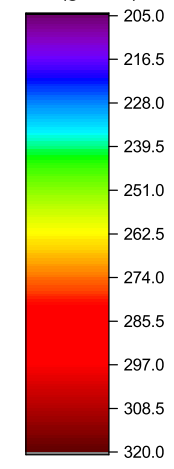

Figure 5.4: Engine BSFC map and operating points for different ICE modes during the HWFET driving cycle. 
Table 5.3

Series E-REV performance results for using a single-mode engine during the HWFET driving cycle

\begin{tabular}{|l|c|c|c|c|}
\hline \hline $\begin{array}{l}\text { Engine } \\
\text { mode }\end{array}$ & $\begin{array}{c}\text { Fuel consumption } \\
\text { (grams) }\end{array}$ & $\begin{array}{c}\text { Ave. engine } \\
\text { BTE }(\%)\end{array}$ & $\begin{array}{c}\text { Engine ON time } \\
(\mathrm{sec})\end{array}$ & $\begin{array}{c}\text { Engine work } \\
(\mathrm{MJ})\end{array}$ \\
\hline \hline HCCI & 1114.2 & 38.6 & 1539 & 18.7 \\
RCCI & 1131.3 & 38.0 & 1279 & 18.7 \\
SI & 1261.5 & 34.7 & 666 & 19.2 \\
\hline
\end{tabular}

area of SI engine is located at high power area (" $P \geq 35 \mathrm{~kW}$ "). As a result the engine ON time (Tables 5.2, 5.3) for the SI engine is significantly less than the HCCI engine. Another point to compare is the variation of the engine optimum power with the driver's demands and vehicle speed (Figure 5.5). From Figure 5.5 it can be seen that the HCCI is generating power for all the range of the power demand and vehicle speed due to its limited power range. However, the SI engine is mostly OFF, for instance, during the braking (i.e., negative power demand). This is because the SI engine's high BTE area is located at the high power regions; thus, the SI engine's ON time is significantly smaller compared to the HCCI and RCCI engines (Tables 5.2 and 5.3 ). Figure 5.5 is presented here for comparing the engines' optimum power as a function of the vehicle speed and power demand. It should be noted that the battery SOC values will affect the engine optimum power values in Figure 5.5 ,

In addition, Tables 5.2 and 5.3 show that the HCCI has 9\%-10\% better average BTE compared to the SI engine. Since the HCCI engine's best BSFC is located at lower power (" $\leq 10 \mathrm{~kW}$ "), it has $15 \%$ less engine work compared to the SI engine. 

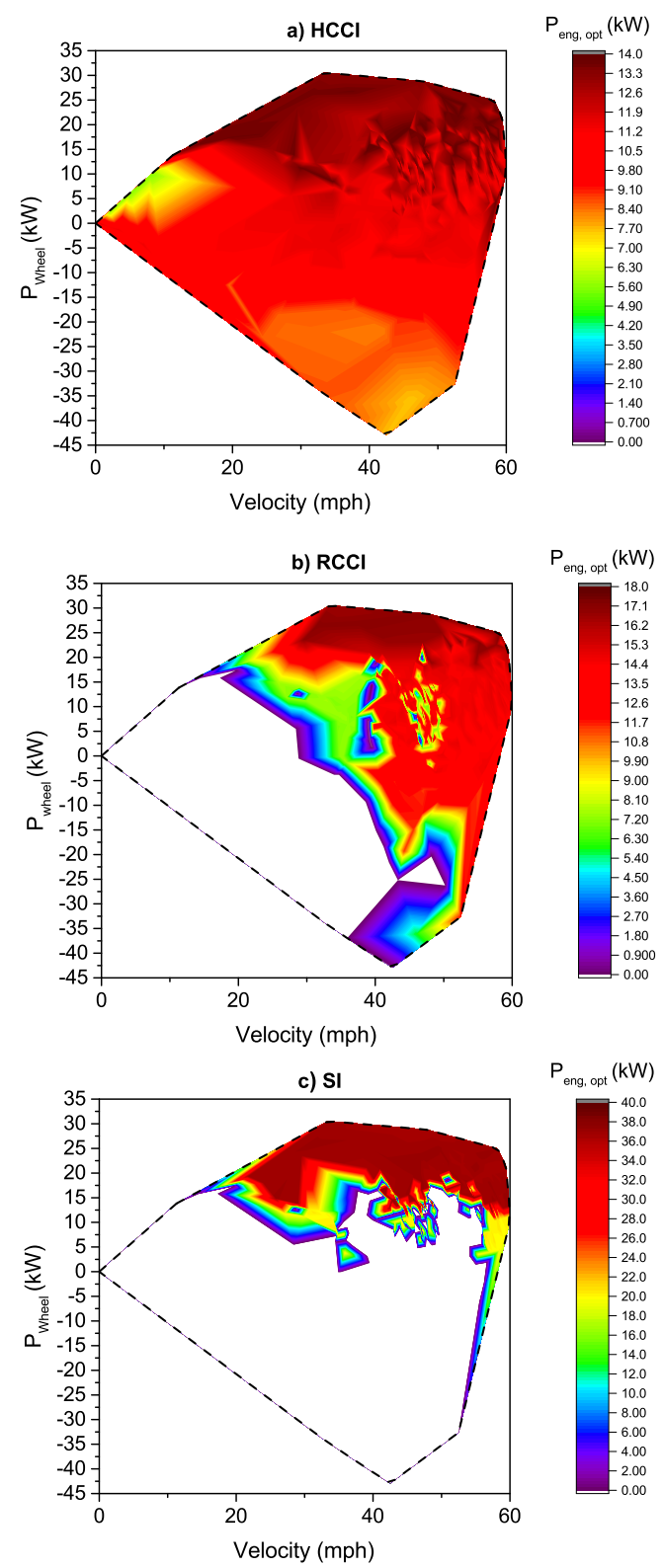

Figure 5.5: Optimum engine power $\left(P_{\text {eng,opt }}\right)$ of the a) HCCI, b) RCCI, and c) SI engines based on the vehicle speed and wheel power for the HWFET driving cycle. The white regions inside dashed lines show the conditions when the engine is OFF. 
Moreover, the hybridization benefit comes from saving the regenerative power back to the battery, and taking advantage of the extra degree of freedom to operate the engine and electrical motor at their best available efficient points. Assuming the battery SOC is balanced at the end of the cycle, performance of the PMP optimization method is reflected on the engine efficiency and engine positive work. For both UDDS and HWFET cycles the PMP method was able to push the engine operating points close to the maximum efficiency areas for all engines (Figure 5.4).

Figure 5.6 shows the wheel, engine and battery works for four different modes of positive and negative wheel and battery power. As expected the vehicle does not perform in mode 3 in which the battery discharges when the wheel power is negative. As can be seen from Figure 5.6, most of the SI engine power is in mode 2, where the wheel power is positive and battery power is negative; that is the engine not only provides the wheel power, but also charges the battery with high power. However, in HCCI engine almost $30 \%$ of engine work is in mode 1 , in which battery and engine provide the wheel power together. This emphasizes the benefit of series E-REV-HCCI engine in city such as UDDS since the engine can provide the wheel power while operating in its high efficient areas. The SI engine, however, has to pay the price of charging/discharging battery loss in order to be able to operate in high efficient region. In fact, the amount of battery power consumed in mode 1 should be returned to the battery in modes 2 and 4 . The best engine-cycle match would be the one that the powertrain can operate in its best efficiency area and the battery power in mode 2 
is close to zero, meaning the battery discharges the same amount of power which was charged during vehicle deceleration. As can be seen from Figure [5.6, the distribution of engine work in modes 1 and 2 for RCCI and HCCI is close to each other, which agrees with the fact that they don't provide any substancial benefit on engine work over the other one (Tables 5.2 and 5.3). As a result, the fuel consumption benefit of HCCI over RCCI is solely based on its higher engine efficiency.

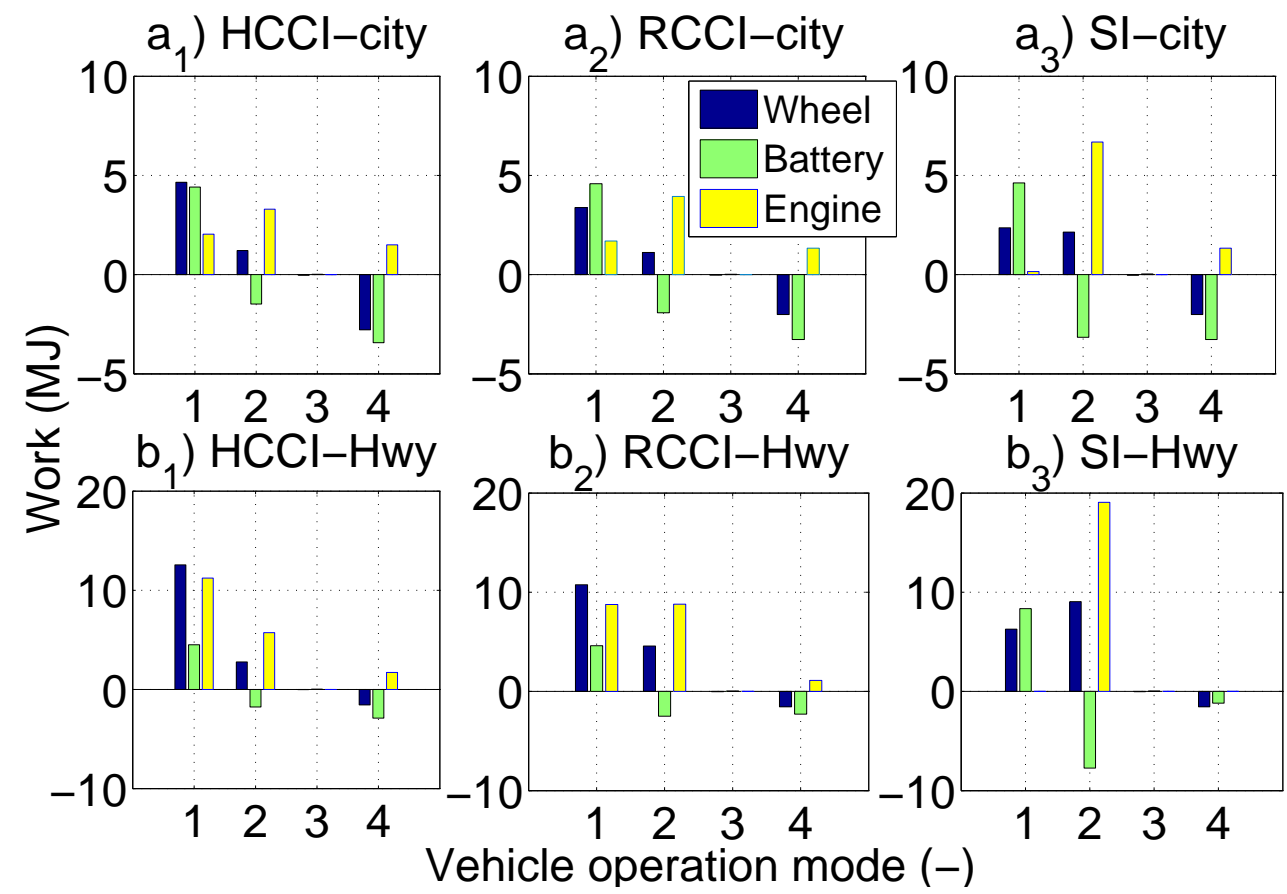

Figure 5.6: Total work of the vehicle at wheels, the battery, and the single-mode engine for the UDDS (top row) and the HWFET driving cycles (bottom row). The x-axis is defined as: 1) $P_{\text {wheel }}>0$ and $P_{b a t}>0,2$ ) $P_{\text {wheel }}>0$ and $P_{b a t}<0$,3) $P_{\text {wheel }}<0$ and $P_{b a t}>0$,4) $P_{\text {wheel }}<0$ and $P_{b a t}<0$.

Figure 5.7 shows the fuel economy of the dedicated single-mode engines in the series E-REV powertrain over the UDDS and HWFET driving cycles. The highest fuel 
economy value belongs to the single-mode HCCI engine over the UDDS driving cycle. Whereas, the single-mode SI engine has the lowest fuel economy over the HWFET driving cycle. Moreover, Figure 5.7 shows the fuel economy improvement of the dedicated single-mode HCCI and RCCI engines over the SI engine. It can be seen for the city driving cycle that the fuel economy improvement is $12.0 \%$ and $9.0 \%$ for the HCCI and RCCI engines, respectively. These numbers are higher for the highway driving cycles since the LTC engine ON time is higher in the highway cycle, which gives more opportunity to the LTC engine to save more fuel.

(a)

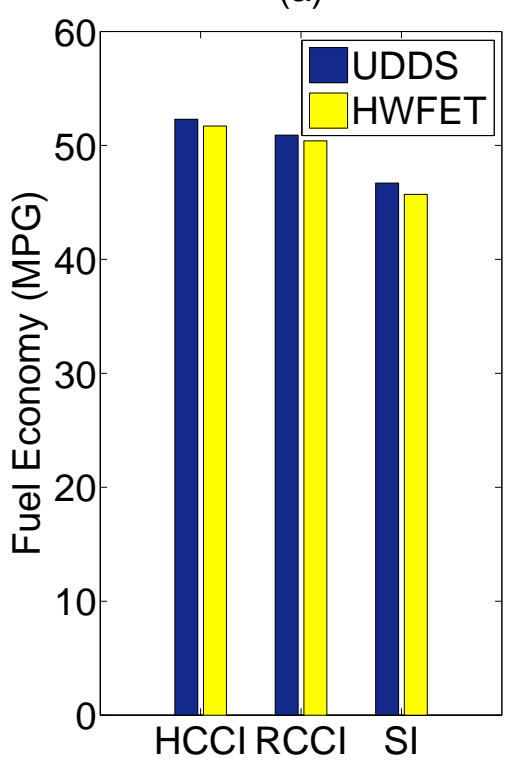

(b)

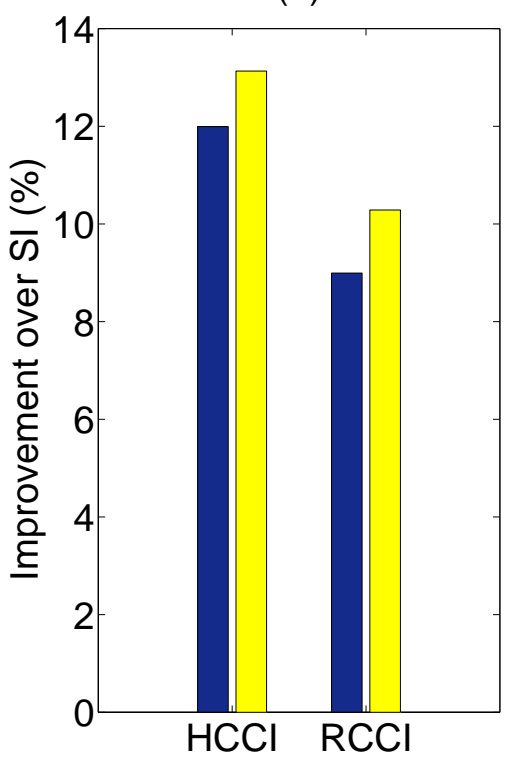

Figure 5.7: Fuel economy of the series E-REV running with different single-mode engines (left) and fuel economy improvement over the SI engine (right). The results are for UDDS and HWFET driving cycles.

Figure 5.8 shows the engine speed versus the vehicle speed for different engines. As can be seen from Figure [5.8, the SI engine speed can reach $3000 \mathrm{rpm}$ for vehicle 
velocity as low as $30 \mathrm{mph}$. High engine speed at low vehicle velocity is not favorable from the NVH (Noise, Vibration and Harshness) stand point. Whereas, all the RCCI and HCCI operating points are in the favorable noise zone. Restricting the SI engine from running in the high noise region enforces the engine to operate in low efficiency regions. This means that the fuel economy of the SI engine decreases and this results in increasing the HCCI and RCCI engines fuel economy advantage over the SI engine. This behavior can also be observed in Figure 5.6, where for the case of SI engine, most of engine power is at mode 2 (i.e., $P_{\text {wheel }}>0$ and $P_{b a t}<0$ ), with the positive wheel power and negative battery power. This means during the time when the vehicle is either at EV mode or the engine is operating at high power driving the wheels as well as charging the battery.

\subsubsection{Multi-mode Engine}

In the previous subsection the single-mode engines were discussed and no modeswitching was allowed among the different engine modes. For multi-mode engine operation, $\Lambda$ in Equation (6.15) is changed to "1", which accounts for the fuel penalty associated with mode-switching $\left(m_{i j}\right)$. The mode-switching is defined to enable all the possible switches between different modes including SI $\leftrightarrow \mathrm{HCCI}, \mathrm{RCCI} \leftrightarrow \mathrm{SI}$, and HCCI $\leftrightarrow$ RCCI. The baseline fuel penalty for mode-switching is based on the results

of the study in [91]. The PMP balances different costs including the mode-switching 
(a) UDDS

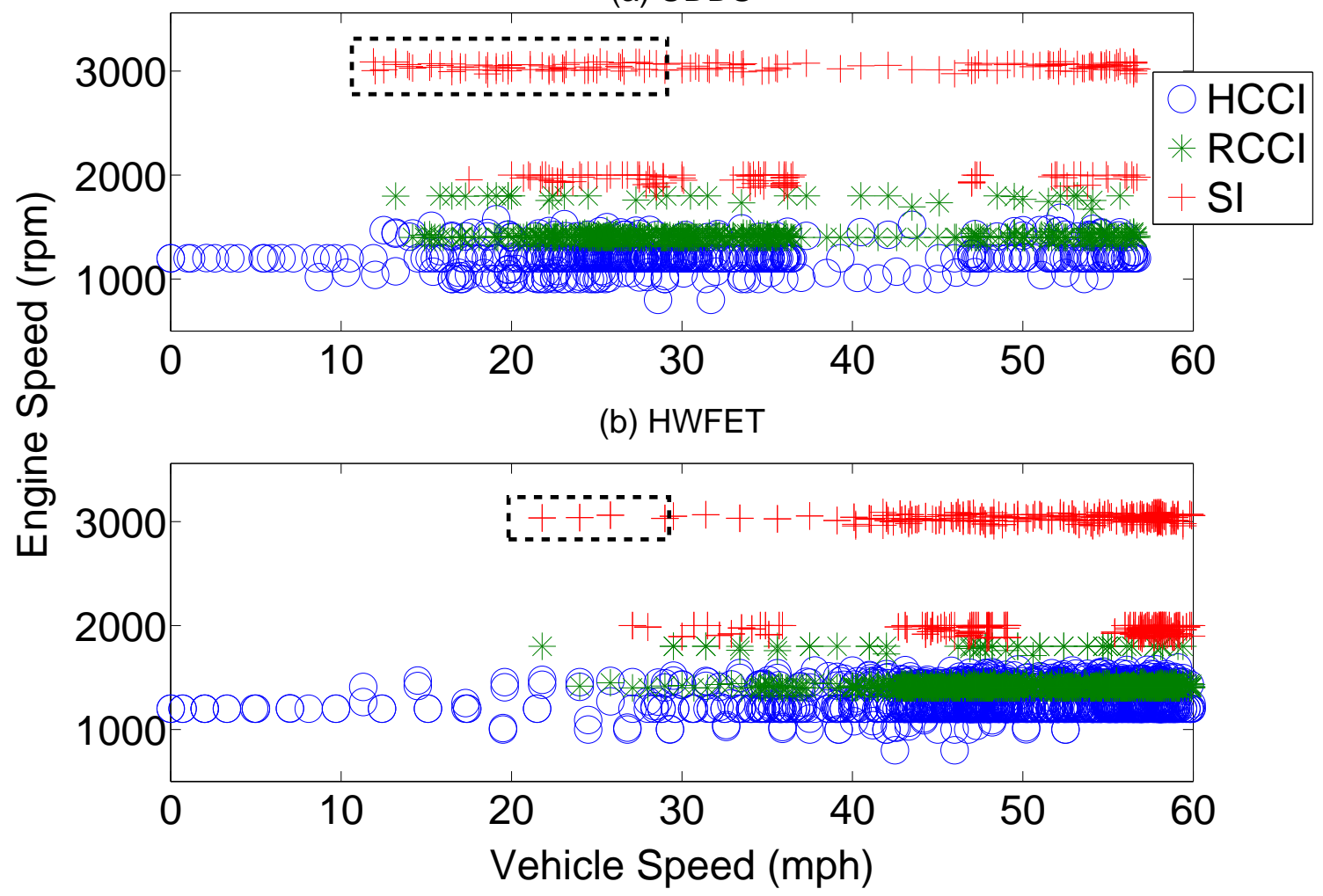

Figure 5.8: The engines' speed versus vehicle speed for the FT75 driving cycle. High noise area is shown by a dashed box in the subplots.

fuel penalty to develop a charge-sustaining control strategy. Figures 5.9 and 5.10 show the fuel economy variation of the multi-mode series E-REV with the $m_{i j}$ in both UDDS and HWFET driving cycles. The effect of the $m_{i j}$ compared to the baseline is investigated. The $m_{i j}$ to switch to the SI and RCCI modes are varied from the baseline value to $5 \%$ of the baseline $m_{i j}$ and the results are shown in Figures [5.9 and 5.10, respectively. 


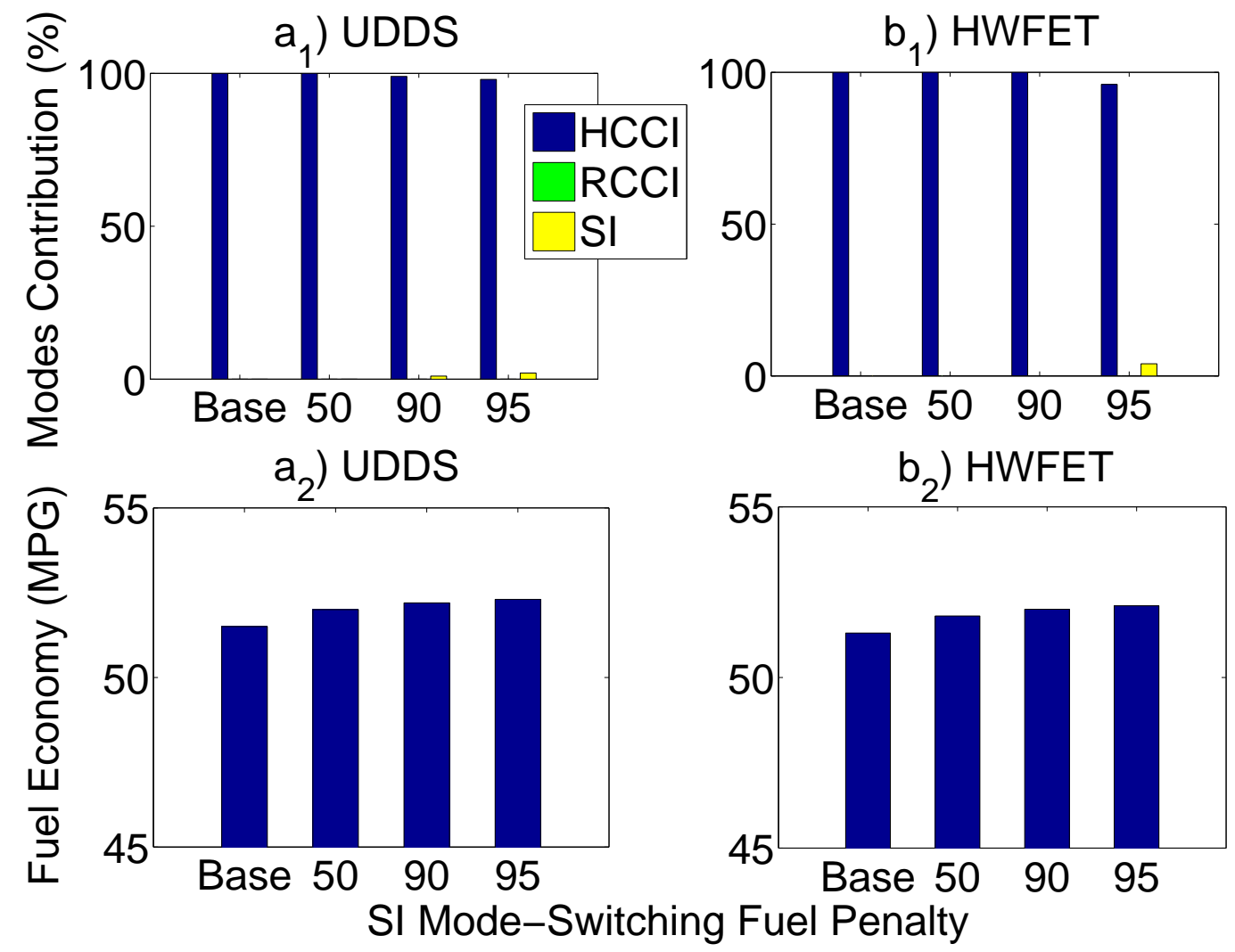

Figure 5.9: Optimized multi-mode engine operation and effect of SI mode switching fuel penalty on the vehicle fuel economy and engine mode contributions. The results are shown when the fuel penalty is reduced by 50,90 , and 95 percent compared to the baseline.

As can be seen from Figure [5.9, reducing the fuel penalty for switching to SI does not improve the vehicle fuel economy. The HCCI is the engine dominant mode and when the fuel penalty for switching to SI mode decreases by 95 percent the engine operates 3 percent of its ON time in the SI mode. The SI mode operation time will increase in high power demand driving cycles. This can be explained by the fact that the SI engine is more efficient at high power regions and the HCCI and RCCI engine have limited power range (Figure 5.4). Moreover, the PMP optimizer chooses to operate 
the engine in HCCI mode rather than RCCI mode due to the higher efficiency of the HCCI engine.

In Figure 5.10 the sensitivity of the results with varying the fuel penalty for modeswitch to RCCI is demonstrated. Again the HCCI is the dominant engine operating mode for the both UDDS and HWFET driving cycles. However, the RCCI mode consists of 45 percent of the engine operating modes when the fuel penalty to the RCCI decreases by 95 percent relative to the baseline. Moreover, the vehicle fuel economy increases 2 percent by including all of the engine modes in the series E-REV powertrain in comparison to the best single-mode fuel penalty (Figure 5.10). 


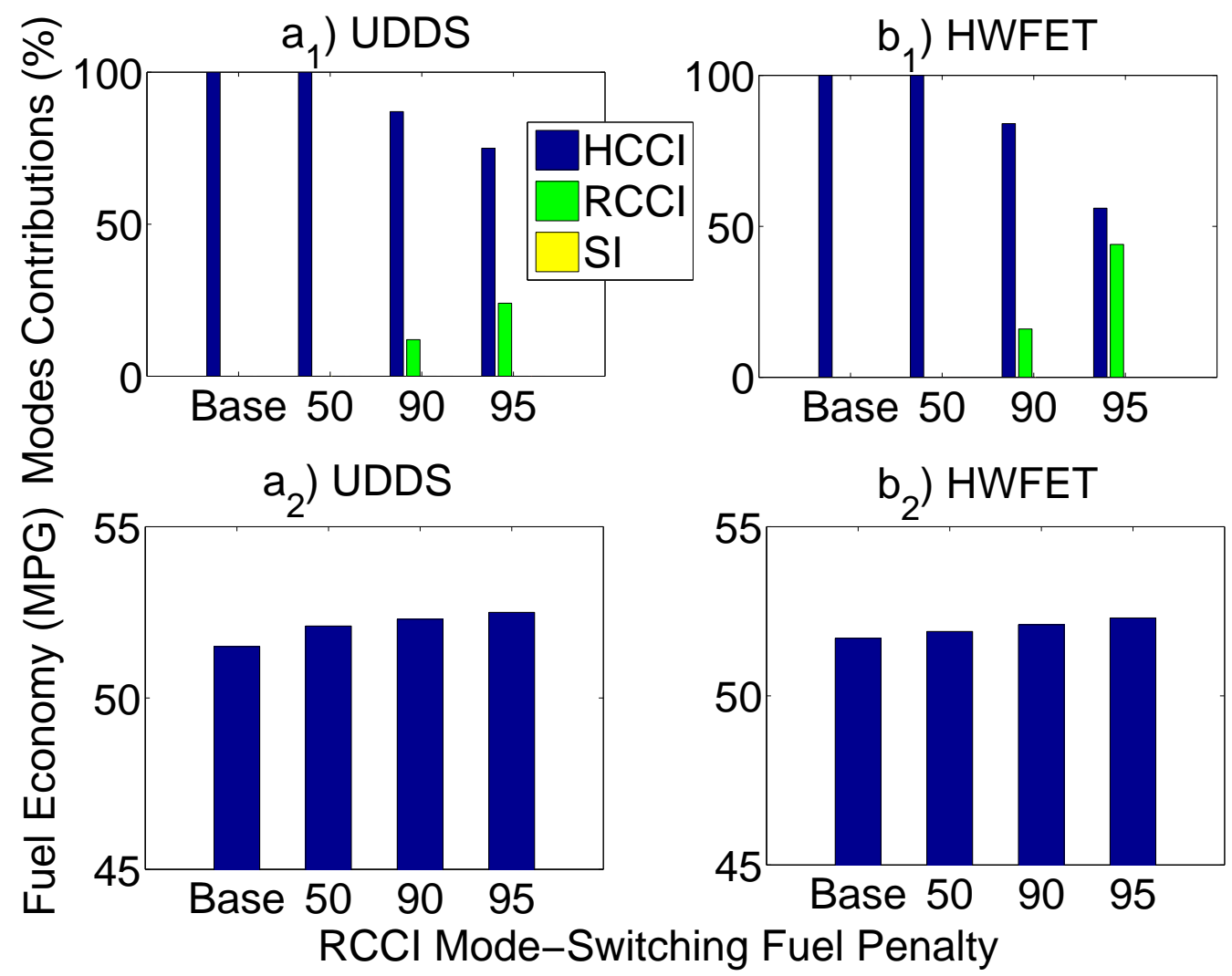

Figure 5.10: Optimized multi-mode engine operation and effect of $R C C I$ mode switching fuel penalty on the vehicle fuel economy and engine mode contributions. The results are shown when the fuel penalty is reduced by 50, 90, and 95 percent compared to the baseline.

The multi-mode engine fuel economy benefit is affected by the type of driving cycle and the fuel penalty values for mode-switching. The mode-switching fuel penalty relies on the engine combustion controller performance. The results in Figures [5.9 and 5.10 show that the multi-mode series E-REV can improve the fuel economy of the vehicle more than the single-mode series E-REV. By reducing the baseline mode-switching fuel penalty the multi-mode operation becomes even more promising; however, this requires advanced model-based combustion control strategies for 
switching to the different engine modes. In that case, the HCCI+RCCI modes become the best engine operating modes for low and mid power range driving cycles and the RCCI+SI is the best combination for more aggressive driving cycles (i.e., higher power demand).

\subsection{Conclusions}

Fuel economy potential of utilizing a multi-mode LTC engine in a series E-REV was investigated. To this end, a hybrid electric powertrain setup was designed and built to provide required experimental data. The PMP optimal control technique was employed and vehicle model/maps were extracted using collected experimental data from the hybrid electric powertrain setup. The powertrain setup is able to perform different levels of LTC engine hybridization. The PMP framework aimed to investigate the ultimate fuel saving of a series E-REV powertrain with multi-mode LTC engine. Below are the main findings based on the optimization results in this chapter:

- The simulation results for the UDDS cycle show the single-mode HCCI and RCCI engines offer up to $12.0 \%$ and $9.0 \%$ fuel economy improvement, respectively over a single-mode SI engine in the series E-REV. These improvements increase to $13.1 \%$ and $10.3 \%$ in the HWFET driving cycles for the HCCI and RCCI engines over the 
SI engine. This is due to longer engine $\mathrm{ON}$ time in the high power demand driving cycles. This provides more opportunity for the HCCI and RCCI engines to save more fuel compared to the SI engine.

- The HCCI and RCCI engines are more favorable from the NVH point of view since their operating points are at low engine speed region, assuming combustion noise is controlled well in HCCI and RCCI engines. These engines have higher efficiency than the SI engine at relatively low engine speed, while at the low vehicle speeds the SI engine operates at high engine speeds, which creates harsher noise. By applying the NVH constraint in selecting the engine operating points, the HCCI fuel economy improvement over the SI engine can go beyond the reported $12 \%$ in this chapter.

- Integrating a multi-mode LTC engine in the series E-REV offers $2 \%$ more fuel economy improvement compared the best fuel economy for the single-mode LTC engine in this chapter. This improvement depends on the type of driving cycle. High power demand driving cycles show higher fuel economy improvement.

- Reducing the mode-switching fuel penalty will increase the fuel economy of the series E-REV with the multi-mode LTC engine. Among the engine modes, HCCI is the dominant engine operating mode for the powertrain setup in this chapter. If the fuel penalty to the RCCI mode decreases by 95 percent, the engine operates near to $45 \%$ of its run time in the RCCI mode. 



\section{Chapter 6}

\section{Multi-Mode LTC-SI in P2 Parallel HEV Architecture 1}

In this chapter, the experimentally developed multi-mode LTC-SI engine from chapter 4 is integrated with a parallel hybrid electric configuration, where the engine operation modes include homogeneous charge compression ignition (HCCI), reactivity controlled compression ignition (RCCI), and conventional spark ignition (SI). The powertrain controller is designed to enable switching among different modes, with minimum fuel penalty for transient engine operations. A Pontryagin's Minimum Principal (PMP) methodology is used in the energy management supervisory controller to study a multi-mode LTC engine in parallel HEV architecture with various

${ }^{1}$ The results in this chapter are based on the submitted journal paper in [3] with permission from Elsevier as shown in Appendix D 
hybridization levels. The amount of torque assist by the e-motor can change the LTC mode operating time, which leads to variation in the vehicle's fuel consumption. The results for the UDDS driving cycle show the maximum benefit of the multimode LTC-SI engine is realized in the mild electrification level, where the LTC mode operating time increases dramatically from 5.0\% in Plug-in Hybrid Electric Vehicle (PHEV) to $20.5 \%$ in mild HEV.

\subsection{Introduction}

Figure 6.1 shows the updated summary of prior studies in the literature. For brevity, only new parts are explained here, as other parts were previously explained in chapters 2, 3, and 5 , 


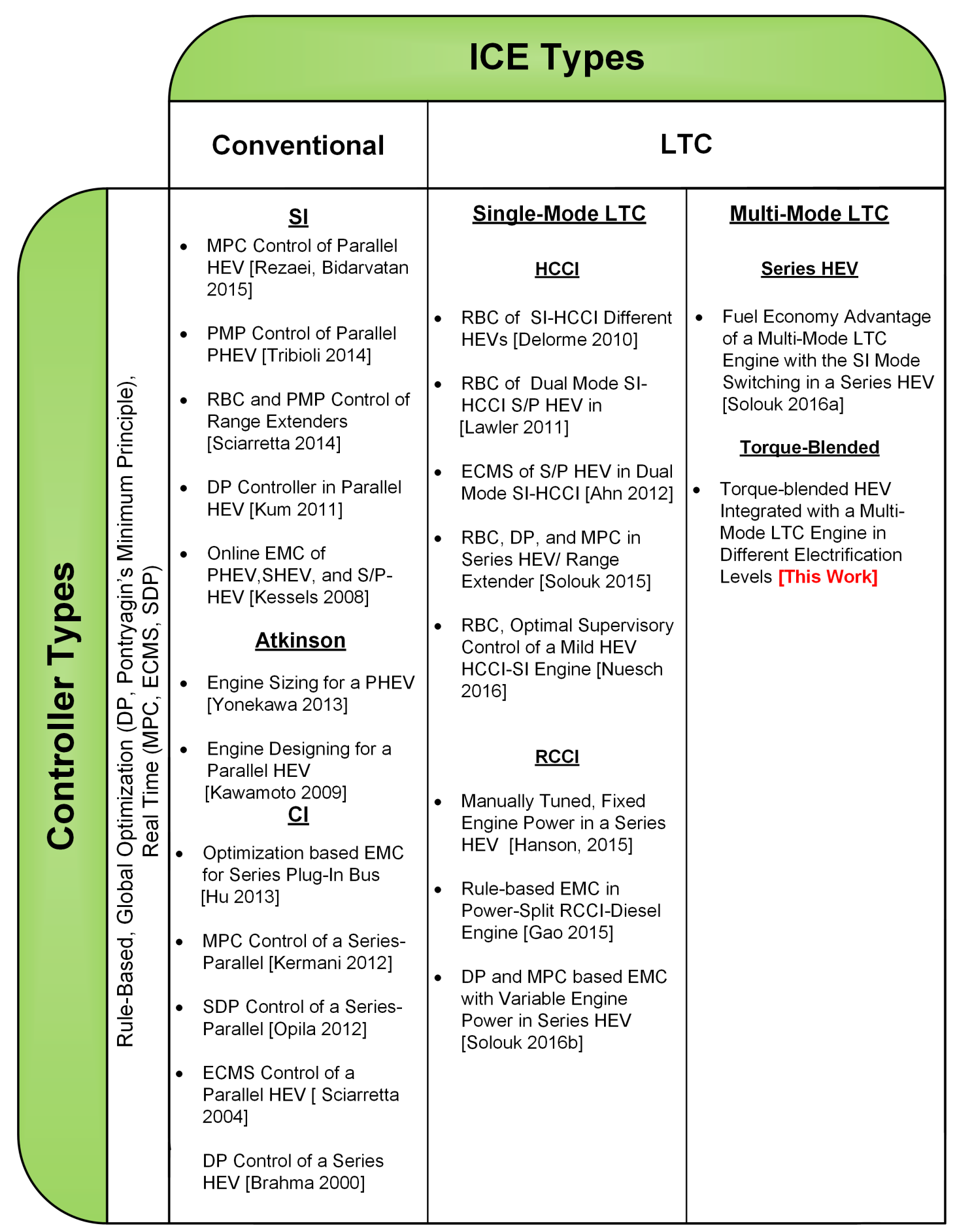

Figure 6.1: Different types of ICEs and control techniques used in HEVs in previous studies. 
In previous chapter, in reference [10], a multi-mode LTC engine was designed to switch between the HCCI, RCCI and conventional SI modes. The results show a $9 \%$ to $13.1 \%$ fuel economy improvement, compared to an identical series HEV platform running with a single-mode SI engine.

This chapter presents the first study undertaken to investigate the fuel economy benefit of integrating a multi-mode LTC engine with a parallel HEV with an advanced optimal control strategy and incorporating required exhaust gas temperature to enable high conversion efficiency for exhaust aftertreatment systems. The LTC modes in this chapter include both HCCI and RCCI modes. The contribution from this chapter is threefold: First, it investigates the fuel consumption reduction of a multimode LTC-SI in a parallel HEV configuration by considering the emission constraints. Second, it investigates the effect of the hybridization level on the fuel saving over the single-mode SI in the parallel HEV. Third, it examines the optimum engine operating modes under different vehicle loads and speeds.

This chapter is organized in five sections. In the following section, the optimal control problem for a parallel HEV architecture is formulated and pontryagin's minimum principle (PMP) optimization techniques are applied. Then, the results of the parallel $\mathrm{HEV}$ for the single-mode and multi-mode engine operations are discussed. Finally, all findings from this chapter are summarized in the last section and conclusions are drawn. 


\subsection{Design of Optimal Energy Management Con- trol Strategy}

The goal of the optimal control in this chapter is to minimize the total energy consumption provided by the battery and fuel. In this chapter the charge-sustaining mode is considered to enable comparing fuel consumptions in different cases. Given the battery total energy will be zero in the charge-sustaining mode, the cost function is defined as the total vehicle fuel consumption $\left(\dot{m}_{f}\right)$ by Eq. (6.1):

$$
J(u(t))=\int_{0}^{T} \dot{m}_{f}(t) d t
$$

where, $\dot{m}_{f}$ is the rate of the engine fuel consumption and $T$ is the time length of a driving cycle. Equation (2) shows the constraints for the HEV optimization problem. 


$$
\begin{aligned}
& \left|S O C_{f}-S O C_{0}\right| \leqslant \epsilon \\
& S O C_{\text {min }} \leqslant S O C(t) \leqslant S O C_{\text {max }} \\
& P_{\text {bat,min }}(S O C) \leqslant P_{\text {bat }}(t) \leqslant P_{\text {bat }, \text { max }}(S O C) \\
& T_{\text {eng,min }}\left(\omega_{\text {eng }}\right) \leqslant T_{\text {eng }}\left(t, \omega_{\text {eng }}\right) \leqslant T_{\text {eng,max }}\left(\omega_{\text {eng }}\right) \\
& \omega_{\text {eng,min }} \leqslant \omega_{\text {eng }}(t) \leqslant \omega_{\text {eng,max }} \\
& T_{\text {motor,min }}\left(\omega_{\text {mot }}\right) \leqslant T_{\text {motor }}(t) \leqslant T_{\text {motor,max }}\left(\omega_{\text {mot }}\right) \\
& \omega_{\text {motor,min }} \leqslant \omega_{\text {motor }}(t) \leqslant \omega_{\text {motor,max }} \\
& T_{e m p_{\text {exh }}}\left(\omega_{\text {eng }}, T_{\text {eng }}\right) \geqslant T e m p_{\text {light }- \text { off }}
\end{aligned}
$$

The constraints in Eq. (2) are applied for the battery SOC operation window, battery power $\left(P_{b a t}\right)$, engine torque $\left(T_{\text {eng }}\right)$, engine speed $\left(\omega_{\text {eng }}\right)$, e-motor torque $\left(T_{\text {motor }}\right)$, emotor speed $\left(\omega_{\text {motor }}\right)$, and oxidation catalyst light-off temperature (Templight-off $)$ in the exhaust aftertreatment system. This optimal control problem is solved using optimal control techniques that are described in the following.

\subsubsection{Pontryagin's Minimum Principal (PMP)}

The PMP method is based on a general case of the Euler-Lagrange equation and originates from the calculus of variations. This method yields the necessary - not 
sufficient - conditions of the global optimal solution. The optimal trajectories derived from PMP will be the global optimal solution of the HEV problem if the obtained optimal trajectory is a unique trajectory that meets the necessary and boundary conditions [90]. The necessary condition for the PMP global optimality is explained in [90] and will be briefly explained in Subsection 6.2.1.2, In Subsection 6.2.1.1, the simulation model of the target vehicle is described.

\subsubsection{Parallel P2 Architecture Model}

In the P2 parallel HEV architecture, the engine is coupled to the e-motor through a clutch. The output shaft is connected to the drivetrain where an automatic six speed transmission connects the output shaft to the wheels. This limits the engine operating points to discrete gear ratio options. Figure 6.2 shows the parallel HEV architecture and Table 6.1 lists the vehicle parameters along with the transmission ratios used in this chapter. The operating maps and model parameters for the vehicle components are obtained from the experimental setup as previously explained in chapter 4. 


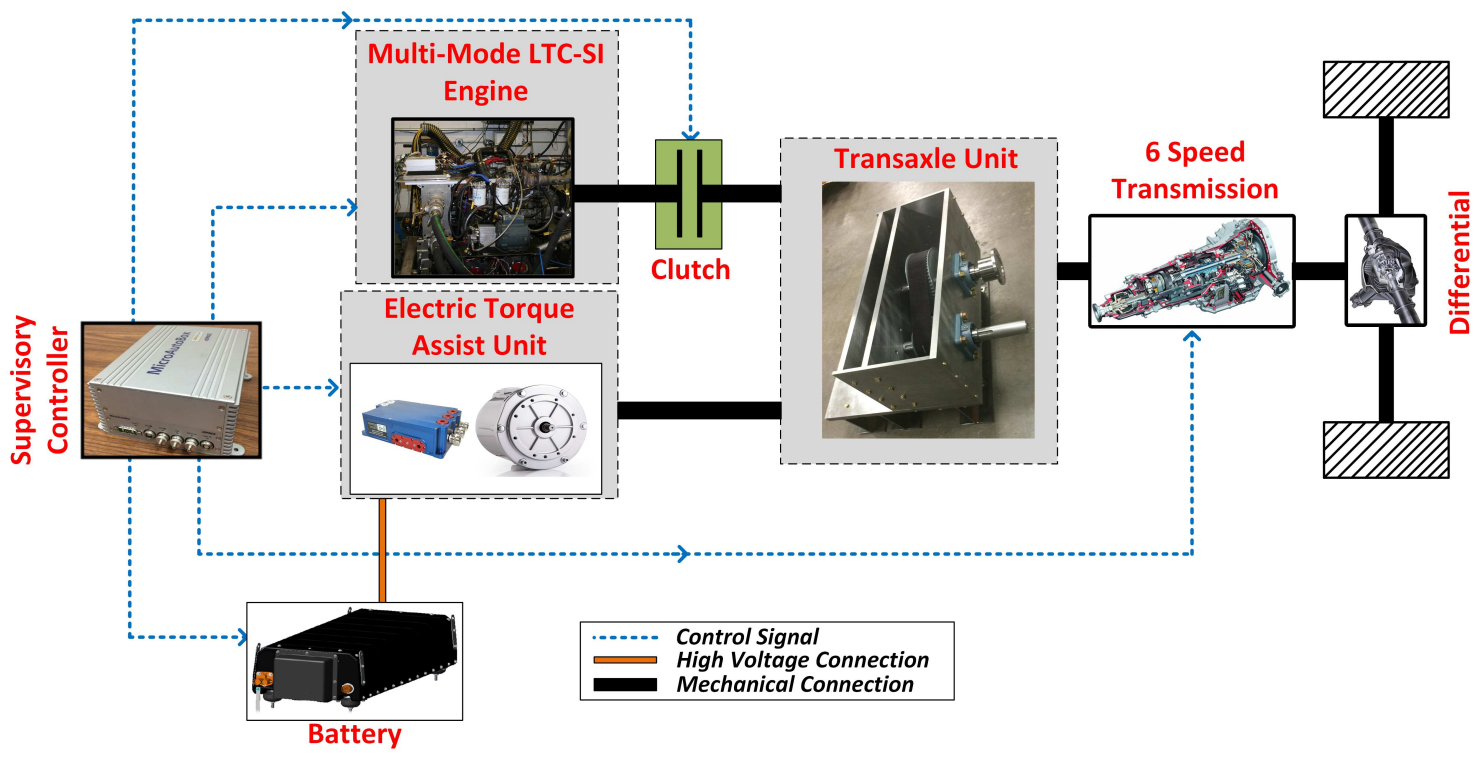

Figure 6.2: Parallel HEV architecture in this chapter.

Table 6.1

Vehicle specifications.

\begin{tabular}{|l|c|}
\hline Parameters & Values \\
\hline \hline Vehicle Curb Mass, $M$ & $1775(\mathrm{Kg})$ \\
Frontal Area, $A$ & $2.0\left(\mathrm{~m}^{2}\right)$ \\
Engine Motor Coupling Gear Ratio, $n_{c}$ & $2.0(-)$ \\
6-Speed Transmission Gear Ratios & {$[3.166,2.05,1.481,1.166,0.916,0.725](-)$} \\
Differential Ratio, $n_{d}$ & $4.529(-)$ \\
Wheel Radius, $r$ & $0.33(\mathrm{~m})$ \\
Drag Coefficient, $C_{d}$ & $0.25(-)$ \\
Rolling Resistance Coefficient, $f_{r}$ & $0.01(-)$ \\
\hline
\end{tabular}

In the parallel HEV in this chapter, there are four independent control variables. These variables include the engine torque, the battery power, the transmission gear ratio, and the clutch status for a given driver's power request and vehicle speed. Both 
the engine and e-motor speeds can be obtained for a given transmission gear ratio and vehicle speed. The e-motor and engine speeds are determined by Eq. (6.3), as a function of the vehicle's speed and the transmission gear ratios as follows:

$$
\left\{\begin{array}{l}
\omega_{m o t o r}=\frac{V_{v e h} \cdot n_{t} \cdot n_{d}}{r} \\
\omega_{\text {eng }}=\frac{\omega_{\text {motor }}}{n_{c}}
\end{array}\right.
$$

where, $V_{v e h}$ is the vehicle speed, $n_{t}$ is the transmission gear ratio, $n_{d}$ is the differential ratio, $r$ is the wheel radius, and $n_{c}$ is the engine/e-motor coupling ratio. In addition, for a given driver's power request, the required engine power can be calculated by Eq. (6.4) to meet the driver's demand.

$$
P_{\text {eng }}=P_{\text {wheel }}-P_{\text {motor }, \text { mech }}
$$

where, $P_{\text {motor, } m e c h}$ is the e-motor mechanical power. Since the battery is supplying the e-motor power, the $P_{\text {motor,mech }}$ can be obtained by knowing the battery power and the e-motor efficiency as follows: 


$$
\left\{\begin{array}{l}
P_{b a t}=P_{\text {motor }, e} \\
P_{\text {motor }, \text { mech }}=\frac{P_{\text {motor,e }}}{\left(\eta_{\text {motor }}\right)^{k} \cdot \eta_{\text {gear }}}
\end{array}\right.
$$

where, $P_{\text {motor,e }}$ is the e-motor input (electrical) power; $\eta_{\text {motor }}$ and $\eta_{\text {gear }}$ are the e-motor and the transmission efficiencies, respectively. $k$ equals to "- 1 " when the wheel power is positive and equals to " 1 " when the power at the wheel is negative. The wheel power is determined by the Longitudinal Vehicle Dynamic equations.

From Eq. (6.3) to (6.5), the engine power, motor power, engine speed, and motor speeds are specified by knowing the transmission gear ratio $\left(n_{t}\right)$ and the battery power at each time. The motor and engine torques are determined accordingly.

$$
\left\{\begin{array}{l}
T_{\text {motor }}=\frac{P_{\text {motor }, \text { mech }}}{\omega_{\text {motor }}} \\
T_{\text {eng }}=\frac{P_{\text {eng }}}{\omega_{\text {eng }}}
\end{array}\right.
$$

The fuel consumption rate is determined from the engine BSFC map as a function of $T_{\text {eng }}$ and $\omega_{e n g}$ : 


$$
\dot{m}_{f}=f\left(T_{e n g}, \omega_{e n g}\right)
$$

Finally, the time derivative of SOC is obtained from the battery dynamic equation as follows:

$$
S \dot{O} C(t)=-\frac{1}{Q_{\text {nom }}} \cdot \frac{O C V^{2}-\sqrt{O C V^{2}-4 R P_{b a t}(t)}}{2 R}
$$

where, $Q_{\text {nom }}$ is the battery nominal energy capacity. The equivalent open-circuit voltage $(O C V)$ and internal resistance $(R)$ are functions of SOC. Thus, the $S \dot{O} C$ is a function of $P_{b a t}$ and SOC. In conclusion, the $\dot{m}_{f}$ in Eq. (6.7) and $S \dot{O} C$ in Eq. (6.8) depend on $P_{b a t}$, and $n_{t}$, if the wheel power $\left(P_{\text {wheel }}\right)$ and vehicle speed are given. Moreover, the $P_{b a t}$, and $n_{t}$ are selected as the control variables in the optimization problem.

\subsubsection{Development of PMP-based torque management strategy}

To apply the optimal control theory to the HEV powertrain, the Hamiltonian $(\mathcal{H})$ is defined as follows: 


$$
\mathcal{H}\left(P_{b a t}, S O C, t\right)=-\lambda(t) . g\left(P_{b a t}(t), S O C(t)\right)+\dot{m}_{f}\left(P_{b a t}, n_{t}, t\right)
$$

where, $\lambda$ is called "costate" in the PMP and $g$ is the state equation, which encompasses the battery dynamics (Eq. 6.8). Thus, to calculate the Hamiltonian, first the $\dot{m}_{f}$ and $g$ are determined using control variables, $P_{b a t}$, and $n_{t}$ from Eq. (6.3) to (6.8).

Using the PMP optimization technique, the state equation and costate equation are obtained as:

$$
S \dot{O} C=\frac{\partial \mathcal{H}}{\partial \lambda}
$$

$$
\dot{\lambda}=\frac{\partial \mathcal{H}}{\partial S O C}
$$

From Eq. (6.9) and Eq. (6.11), if $g$ is not a function of $S O C$ then the costate can be considered constant, as explained in the following.

In reference [10], it is shown that the battery $O C V$ and battery resistance $R$ do not vary significantly in the charge-sustaining over the battery $S O C$ range, i.e. from 
0.3 to 0.7 . In that case, the costate stays near the initial value since the $\frac{\partial g}{\partial S O C}$ is negligible, compared to the costate for the whole driving cycle. Thus, the costate expression during the SOC usage window is simplified as:

$$
\dot{\lambda}=\lambda \frac{\partial g}{\partial S O C}=0, \quad \rightarrow \lambda=\text { constant }
$$

Obtaining a constant value for the costate reduces the PMP complexity. For optimality, the following condition should be considered to specify the optimal control variable $P_{b a t}$ and $n_{t}$ at each time step:

$$
\mathcal{H}\left(P_{b a t}^{*}, n_{t}^{*}, S O C^{*}, \lambda^{*}, t\right) \leqslant \mathcal{H}\left(P_{b a t}, n_{t}, S O C^{*}, \lambda^{*}, t\right)
$$

which means that the optimal control variables $P_{b a t}^{*}$ and $n_{t}^{*}$ minimize the Hamiltonian function at the given time. The boundary condition of the final state variable is

$$
S O C\left(t_{0}\right)=S O C\left(t_{f}\right)
$$


The $P_{b a t}^{*}, n_{t}^{*}$, and $\lambda^{*}$ that satisfy Eq. (6.13) and the boundary condition (6.14), determine the optimal $P_{b a t}$ and $n_{t}$ trajectory. If a costate $(\lambda)$ exists that fulfills the condition (6.14), then the PMP provides the 'global optimal' solution [90].

\subsubsection{Extending the cost function for Multi-Mode operation}

The cost function in Eq. (6.1) is revised by including the engine startup, LTC-SI mode-switching, and gear shifting fuel penalties for minimizing the number of engine starts, LTC-SI mode-switching, and gear-shifting. The revised cost function $(J)$ is:

$$
\begin{aligned}
& J(u(t))=\int_{0}^{T}\left(\dot{m}_{f}\left(P_{b a t}, t\right)+\Gamma \cdot F_{p_{1}}+\Lambda \cdot m_{i j}+\Psi \cdot F_{p_{2}}\right) d t \\
& \Gamma=\left\{\begin{array}{lll}
1 \quad & \text { if } \omega_{\text {eng }}(t-1)=0 & \text { and } T_{\text {eng }}(t-1)=0 \\
& \text { and } & T_{\text {eng }}(t) \geqslant \min \left(T_{\text {eng }}(t)\right) \\
0 & \text {, if } T_{\text {eng }}(t) \leqslant \min \left(T_{\text {eng }}(t)\right)
\end{array}\right.
\end{aligned}
$$

where, $\Gamma$ determines the condition for adding the engine startup fuel penalty. $\Lambda$ is zero when the engine is forced to operate in the single-mode regions and $\Lambda$ is " 1 " 
when the mode-switching is allowed. The engine startup fuel penalty $F_{p_{1}}$ is measured $0.15 \mathrm{~g}$ using the data from the experimental setup in chapter 4 . The $F_{p_{2}}$ is the fuel penalty to avoid frequent gear shifting $\left(F_{p_{2}}=0.1 \mathrm{~g}\right)$. $\Psi$ is 1 when $n_{t}(t) \neq n_{t}(t-1)$ and $\Psi$ is zero when $n_{t}(t)=n_{t}(t-1)$.

The $m_{i j}$ is mode-switching fuel penalty to switch from the $i^{\text {th }}$ engine mode to the $j^{\text {th }}$ engine mode. $i \& j \in[1,2,3]$, since the engine can run in HCCI, RCCI, and SI modes. The $m_{i j}$ prevents frequent model-switching between different modes. It accounts for the fuel penalty associated with each mode switching and finds the global optimal solution. The $m_{i j}$ in this chapter is determined by using the experimental fuel penalty map (Figure 4.13) as was explained in the subsection 4.3.2.

The constraints for the optimal control problem are revised based on the components in the powertrain experimental setup in this chapter: 


$$
\begin{aligned}
& \left|S O C_{f}-S O C_{0}\right| \leqslant 0.01 \\
& 0.3 \leqslant S O C(t) \leqslant 0.7 \\
& P_{\text {bat,min }} \leqslant P_{\text {bat }}(t) \leqslant P_{\text {bat,max }} \\
& P_{\text {eng,min }}\left(\omega_{\text {eng }}\right) \leqslant P_{\text {eng }}\left(t, \omega_{\text {eng }}\right) \leqslant P_{\text {eng,max }}\left(\omega_{\text {eng }}\right) \\
& \omega_{\text {eng,min }} \leqslant \omega_{\text {eng }}(t) \leqslant \omega_{\text {eng,max }} \\
& 0 \leqslant P_{\text {motor }}(t) \leqslant 100 \mathrm{~kW} \\
& 0 \leqslant \omega_{\text {motor }}(t) \leqslant 8000 R P M \\
& \text { Temp } p_{\text {exh }}\left(\omega_{\text {eng,min }}, T_{\text {eng,min }}\right) \geqslant 300^{\circ} \mathrm{C}
\end{aligned}
$$

where, a constraint of maximum one percent $\triangle S O C$ variation is considered for the charge-sustaining mode. The controller selects the engine operating regions where the exhaust gas teamperature is greater than the catalytic converter light-off temperature (i.e., $300{ }^{\circ} \mathrm{C}$ [89]) to achieve low tailpipe $\mathrm{HC}$ and $\mathrm{CO}$ emissions. $N O_{x}$ and soot emissions are ultra low in LTC modes [20].

The next section presents the results for the single-mode engine and the multi-mode engine integrated with the parallel HEV powertrain. The PMP optimal control solution is used for the analysis in this chapter. 


\subsection{RESULTS}

The PMP approach explained in the Section 6.2 is utilized to investigate potential energy saving in the multi-mode LTC-SI engine in the parallel HEV configuration. The costate $\lambda$ in the PMP is selected such that the SOC is balanced at the end of the driving cycle, which means the initial and final SOCs are equal. Three different levels of hybridization are defined by the $\frac{P_{b a t}}{P_{e n g}}$ ratio, as listed in Table 6.2. PHEV has the highest electrification level with peak $60 \mathrm{~kW}$ e-motor power and peak $410 \mathrm{~V}$ battery voltage. The battery and e-motor power limit in the full hybrid category is defined as $40 \mathrm{~kW}$; this number reduces to $18 \mathrm{~kW}$ for the mild hybrid.

Table 6.2

Definition of hybridization levels in this chapter

\begin{tabular}{|l|c|c|c|}
\hline Hybridization & $\frac{P_{b a t}}{P_{e n g}}$ & Electric Motor Power $(\mathrm{kW})$ & Operating Voltage $(V)$ \\
\hline \hline PHEV & 1.0 & 60 & $270-410$ \\
Full Hybrid & 0.65 & 40 & $180-270$ \\
Mild Hybrid & 0.30 & 18 & $80-120$ \\
\hline
\end{tabular}

Figure 6.3 shows the single-mode SI engine BSFC map along with the engine operating points at two hybridization levels. The engine optimum operating points are shown for the Urban Dynamometer Driving Schedule (UDDS) and Highway Fuel Economy Test (HWFET) driving cycles. In both UDDS and HWFET driving cycles, the high power engine operating points are located in the low BSFC region (i.e., $\mathrm{BSFC}<240$ 
$\mathrm{g} / \mathrm{kWh}$ ) for the PHEV, while the engine operating points shift to the low torque and high BSFC regions (i.e., BSFC > $260 \mathrm{~g} / \mathrm{kWh}$ ) when the hybridization level decreases to mild hybrid category. In addition, in the mild hybrid, the engine operating points are more dependent on wheel speed and power demand since the e-motor assist torque is more limited. This results in less flexibility for the torque management controller to place the engine operating points to the low BSFC region. However, a higher battery and e-motor power in the PHEV provides more flexibility for the hybrid powertrain to shift the engine operating points to the more efficient engine regions, while maintaining the battery SOC. Hence, the engine ON time, as listed in Table 6.3, reduces to $184 \mathrm{sec}$ in PHEV compared to $385 \mathrm{sec}$ in mild hybrid for the UDDS. In the HWFET driving cycle, as listed in Table 6.4, the engine ON time reduces from 1052 sec to 739 sec by moving from mild hybrid to PHEV. 


\section{a) UDDS}
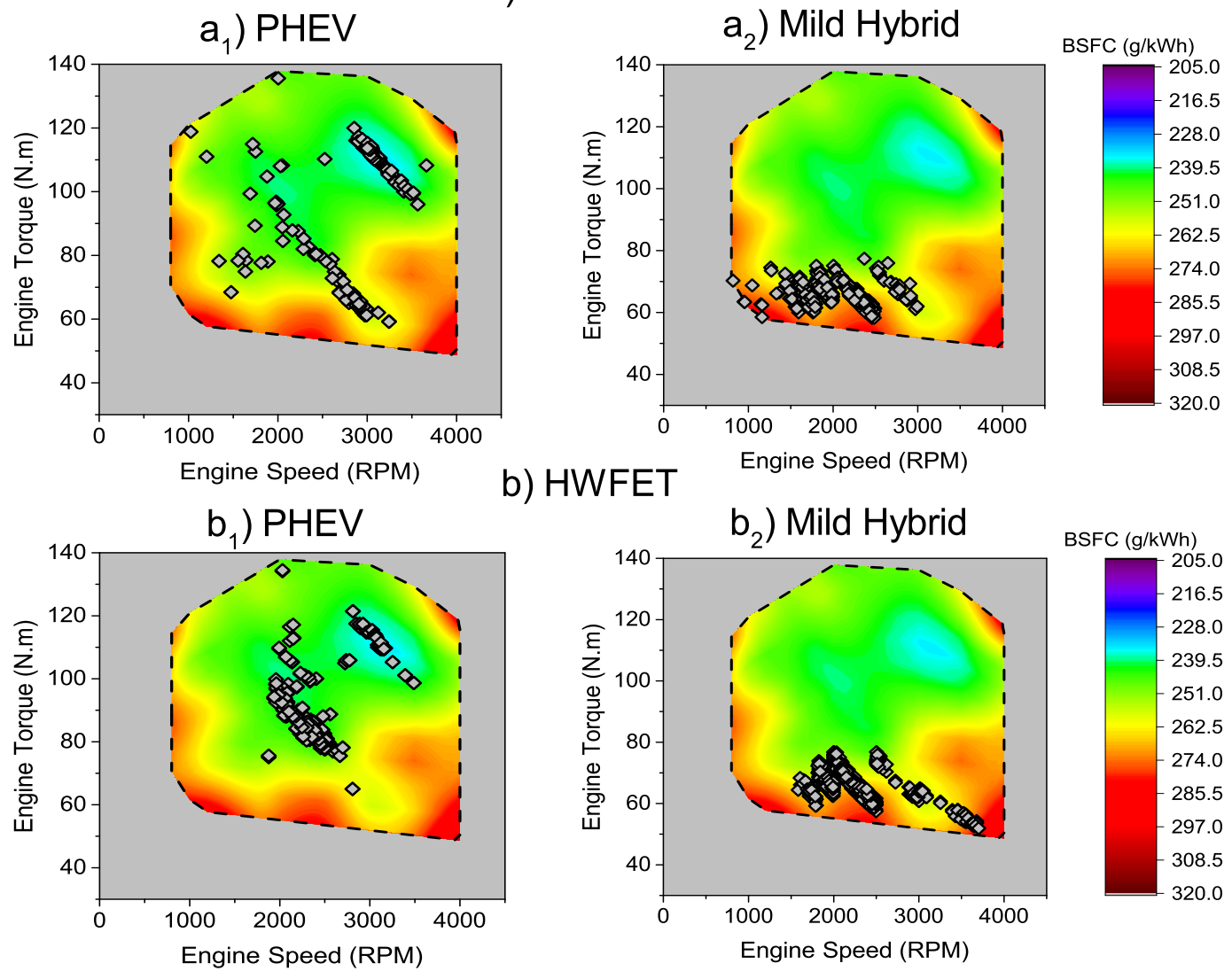

Figure 6.3: Engine operating points over the Single-mode engine BSFC map for different electrification levels for a) UDDS and b) HWFET driving cycles.

Table 6.3

Results for both multi-mode LTC-SI and single-mode SI engine in different electrification levels during UDDS driving cycle

\begin{tabular}{|l|c|c|c|c|c|c|}
\hline \multirow{2}{*}{ Metrics } & \multicolumn{3}{|c|}{ Multi-Mode LTC-SI } & \multicolumn{3}{c|}{ Single-Mode SI } \\
\cline { 2 - 7 } & PHEV & Full Hybrid & Mild Hybrid & PHEV & Full Hybrid & Mild Hybrid \\
\hline \hline Fuel consumption (g) & 356.3 & 344.5 & 378.9 & 365.5 & 358.2 & 409.8 \\
$\mathrm{CO}_{2}$ emission (g) & 1000.2 & 967.1 & 1063.8 & 1026.1 & 996.5 & 1120.0 \\
Ave. engine BTE (\%) & 34.0 & 34.3 & 33.0 & 33.8 & 33.6 & 31.4 \\
Engine work (MJ) & 4.80 & 4.67 & 5.01 & 4.90 & 4.90 & 5.05 \\
Engine ON time (sec) & 233 & 259 & 451 & 184 & 198 & 385 \\
Battery loss (kJ) & 582.3 & 396.8 & 307.1 & 622.6 & 452.0 & 351.6 \\
\hline
\end{tabular}


Table 6.4

Results for both multi-mode LTC-SI and single-mode SI engine in different electrification levels during HWFET driving cycle

\begin{tabular}{|l|c|c|c|c|c|c|}
\hline \multirow{2}{*}{ Metrics } & \multicolumn{3}{|c|}{ Multi-Mode LTC-SI } & \multicolumn{3}{c|}{ Single-Mode SI } \\
\cline { 2 - 7 } & PHEV & Full Hybrid & Mild Hybrid & PHEV & Full Hybrid & Mild Hybrid \\
\hline \hline Fuel consumption (g) & 1168.0 & 1184.3 & 1207.2 & 1170.2 & 1192.9 & 1216.0 \\
$\mathrm{CO}_{2}$ emission (g) & 3324.1 & 3417.7 & 3516.8 & 3361.5 & 3442.7 & 3542.4 \\
Ave. engine BTE (\%) & 34.5 & 34.2 & 31.7 & 34.2 & 34.1 & 31.4 \\
Engine work (MJ) & 16.1 & 16.4 & 15.6 & 16.2 & 16.5 & 15.7 \\
Engine ON time (sec) & 850 & 818 & 1084 & 739 & 798 & 1052 \\
Battery loss (kJ) & 785.3 & 716.6 & 431.6 & 792.1 & 769.8 & 519.0 \\
\hline
\end{tabular}

Figure 6.4 shows the multi-mode LTC-SI engine BSFC map and the engine operating points over the UDDS and HWFET driving cycles. The engine operating points are illustrated for both PHEV and mild hybrid. Comparing Figures 6.3 $a_{2}$ and 6.4 $a_{2}$ shows that the high BSFC operating points in the single-mode engine are now running in the LTC modes (i.e., RCCI, HCCI) in the multi-mode engine. Increasing the running time of LTC modes reduces the overall fuel consumption of the vehicle. LTC modes benefit fuel economy in the mild hybrid vehicle over city driving cycle (i.e., UDDS) the most, since it increases the engine brake thermal efficiency (BTE) without charging the battery. In the PHEV, however, the multi-mode LTC-SI engine has less advantage compared to the mild HEV due to availability of higher electric power for locating the engine operating points in high power SI regions with less engine ON time (see Table 6.3 and Table 6.4). Moreover, in Figure 6.4 $a_{2}$ the engine operates in SI mode over the low engine speeds (i.e., 800 - $1600 \mathrm{rpm}$ ) and mid engine 
torques (i.e., 60 - 80 N.m), while the engine could operate in HCCI mode with a lower BSFC. The optimizer decided to keep the engine on the SI mode in that region due to higher SI-HCCI mode-switching fuel penalty (see Figure 4.13). In addition, in Figure 6.5 $a_{1}$ it can be seen that the engine running time in LTC modes increases by reducing the vehicle electrification level over the UDDS cycle. Among the LTC modes, the RCCI mode has the most engine running time due to the reasons that will be explained later in this section.

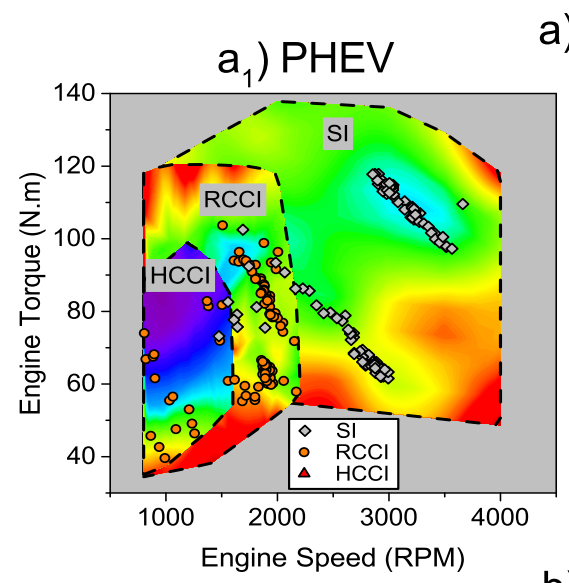

a) UDDS
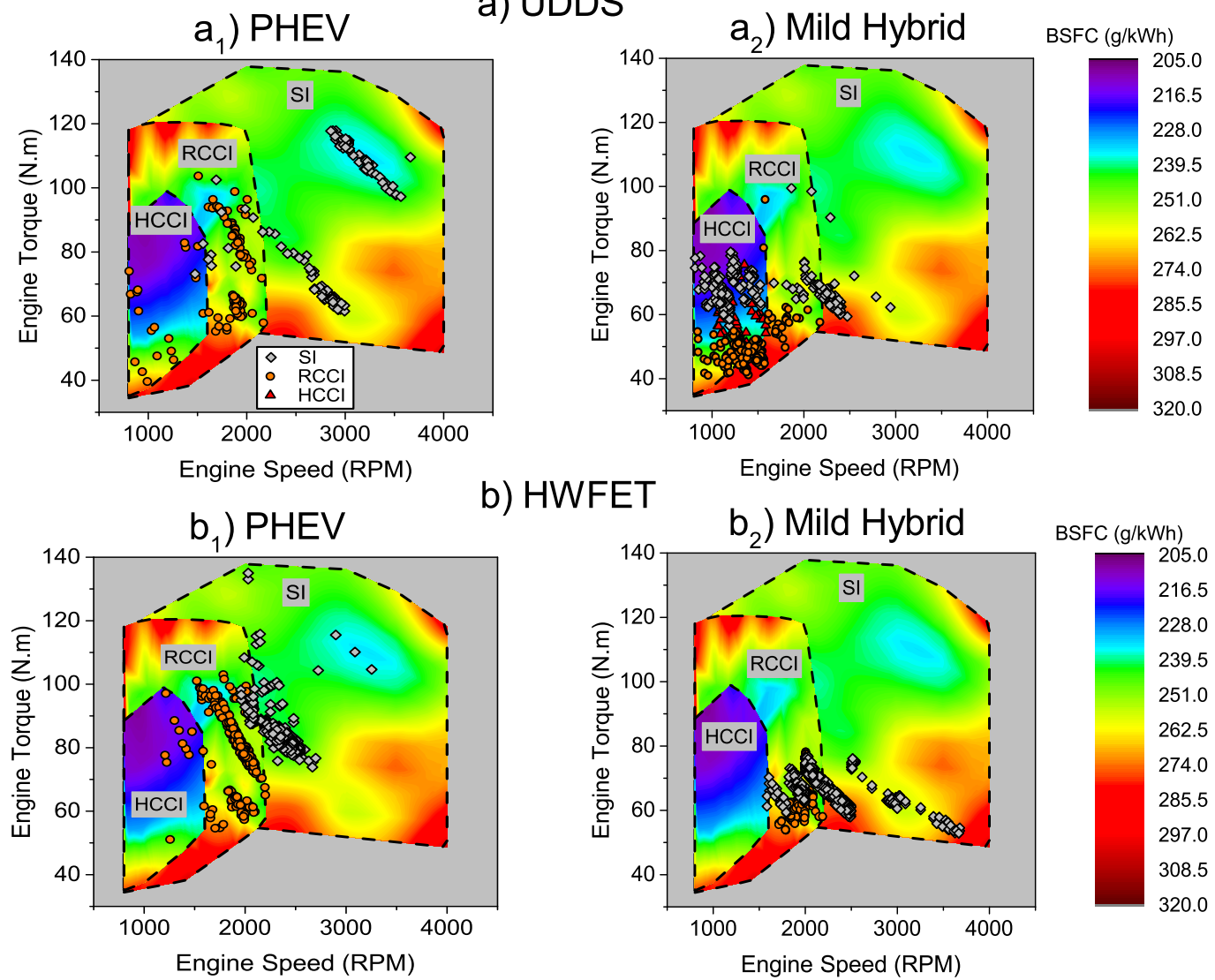

b) HWFET

$b_{2}$ ) Mild Hybrid

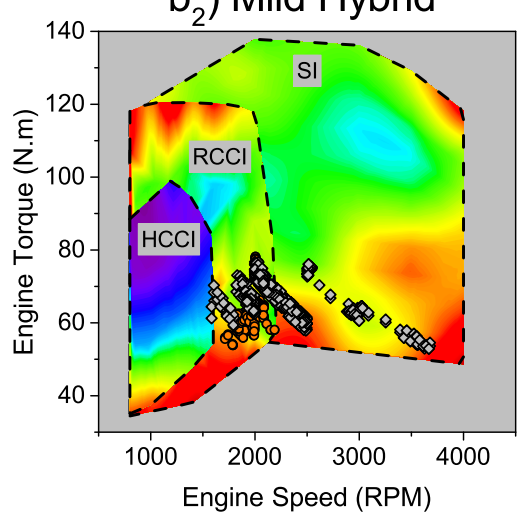

BSFC $(\mathrm{g} / \mathrm{kWh})$

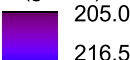

228.0

239.5

251.0

262.5

274.0

285.5

297.0

308.5

320.0

Figure 6.4: Engine operating points over the Multi-mode engine BSFC map for different electrification levels for a) UDDS and b) HWFET driving cycles. 

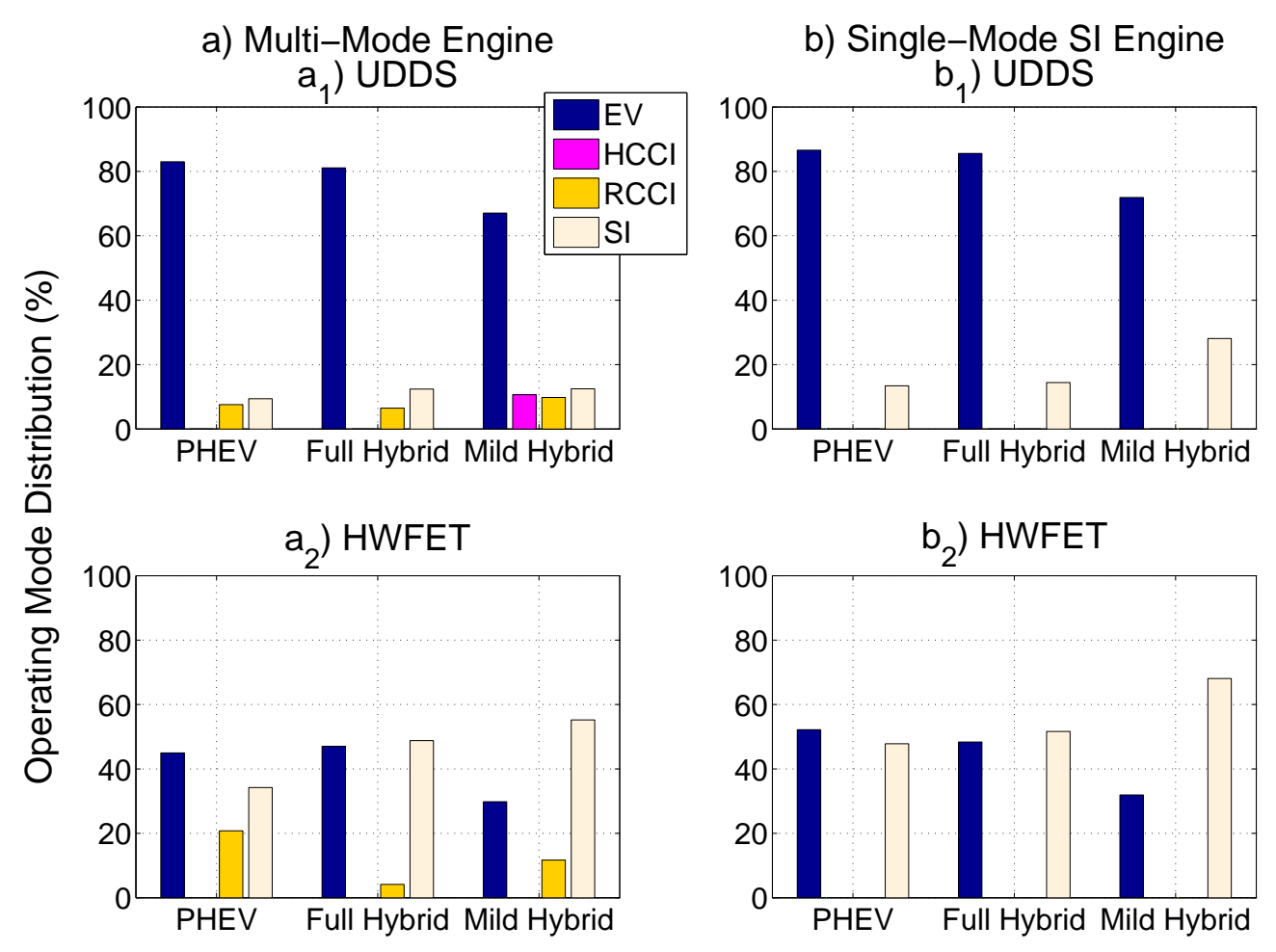

Figure 6.5: Mode distribution in two different driving cycles for three hybridization levels.

A detailed comparison of the engine operating modes in UDDS driving cycle is provided in Table 6.3. The table shows the fuel consumption for different hybridization levels along with the engine work and average engine BTE for each case. The mild hybrid multi-mode LTC-SI case shows a $6.3 \%$ increase in fuel consumption, compared to the PHEV (note: vehicles were run in charge-sustaining mode). Moreover, in the multi-mode mild hybrid, the engine BTE reduces by $3 \%$ and engine work increases by 4.4\%, compared to the multi-mode PHEV. Overall, a comparison between the multimode LTC-SI and single-mode SI shows that the single-mode SI engine has up to 
$7.5 \%$ higher fuel consumption, $4.9 \%$ lower average BTE, and $0.8 \%$ more engine work compared to the multi-mode LTC engine. The lower amount of engine work comes from less utilization of e-motor in single-mode SI, which ends up in lower battery loss on discharging and charging the battery. However, the fuel consumption reduction in the multi-mode LTC-SI compared to the single-mode SI reduces from $7.5 \%$ to $2.5 \%$ by increasing the hybridization level from mild HEV to PHEV.

In addition, Table 6.3 shows that the engine BTE changes less in the multi-mode LTC-SI engine for different hybridization levels, compared to that in the single-mode SI engine. The relatively lower engine $\mathrm{BTE}$ variation (i.e., $\triangle \mathrm{BTE}=1 \%$ ) in the multi-mode case is due to inherent characteristic of the LTC engines in which the high efficiency region is in the low to mid power area with a small BTE variation. With the high power battery in PHEV, the vehicle has flexibility for placing the engine operating points, independent of wheel power demand. As a result, the engine operating points are placed in low BSFC (i.e., BSFC $<233 \mathrm{~g} / \mathrm{kWh}$ ) and high engine power (i.e., $P_{\text {eng }}>30 \mathrm{~kW}$ ) regions to provide the propulsion power as well as charging the battery at its maximum capacity. However, in the multi-mode mild hybrid, the engine operating points cannot be placed in the engine high power region since the battery cannot be charged extensively due to its lower power capacity. Moreover, the engine ON time increases by reducing the electrification level. This also links to the less aggressive battery charge/discharge in the mild hybrid compared to the PHEV; thus, the vehicle electric mode decreases and the engine must run for a longer time 
to provide the demanded power. In the multi-mode engine, however, the availability of low BSFC regions enforces the Hamiltonian function to choose the LTC operating modes, whereas in the single-mode the engine has to operate in higher BSFC regions. The advantage of keeping the engine efficiency close to PHEV is pronounced for city cycles such as the UDDS cycle. For highway cycles such as HWFET, since higher wheel power happens at high vehicle speed $\left(V_{v e h}>80 \mathrm{~km} / \mathrm{h}\right)$, in contrast to city cycles, the HEV controller operates the engine in the best BSFC region of the SI mode with low BSFC values (see Figure6.4 $b_{1}$ ). The engine work differences between PHEV and mild hybrid in Table 6.3 and Table 6.4 are rooted in missing some regenerative power in the mild hybrid (due to battery power limits). Because of more aggressive battery charging/discharging, in general, the PHEV has more battery loss compared to mild hybrid in the both UDDS and HWFET cycles as it is also listed in Tables 6.3 and 6.4. Thus, the multi-mode LTC engine benefits more in the mild hybrid compared to the full hybrid and PHEV.

The vehicle fuel consumption is shown in Figure 6.6 for both UDDS and HWFET driving cycles. The results show the advantage of the multi-mode LTC-SI engine in the mild hybrid over the single-mode SI. However, this improvement rate is smaller over the HWFET driving cycle since the engine operating points are located mainly in fuel-efficient regions independent of the electrification level. 


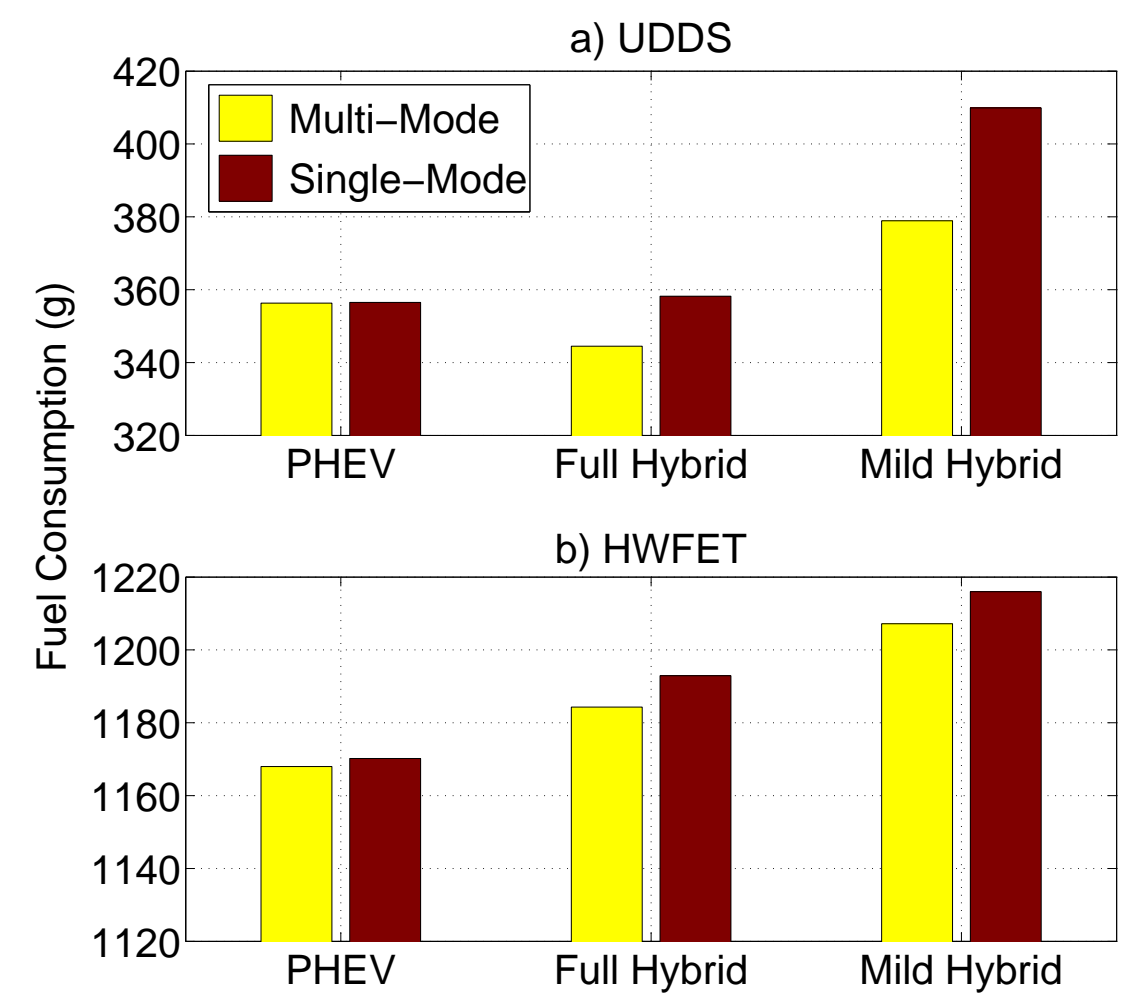

Figure 6.6: Fuel consumption for the multi-mode and single mode engines in two driving cycles and three electrification levels.

Figure 6.7 depicts the powertrain running modes for different hybridization levels for both UDDS and HWFET driving cycles. The x-axis shows the battery power and the y-axis shows the power demand at wheels. The regenerative braking happens once the power sign is negative (i.e., region III). Figures 6.7 $a_{2}$ and $b_{2}$ show that, in the mild hybrid, the $\mathrm{x}$-axis range is smaller for the given demanded wheel power since the available battery power is smaller. Note that the red zone is narrower in mild hybrid compared to PHEV, since it has less tendencies to use the engine in SI mode due to lower battery charging/discharging. 


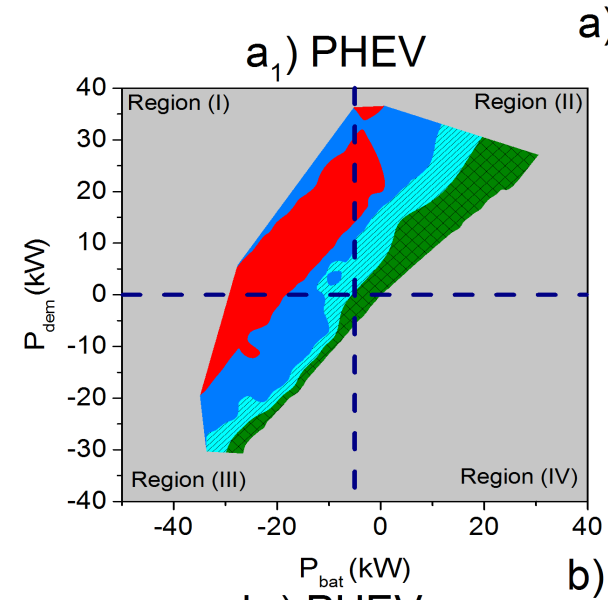

a) UDDS
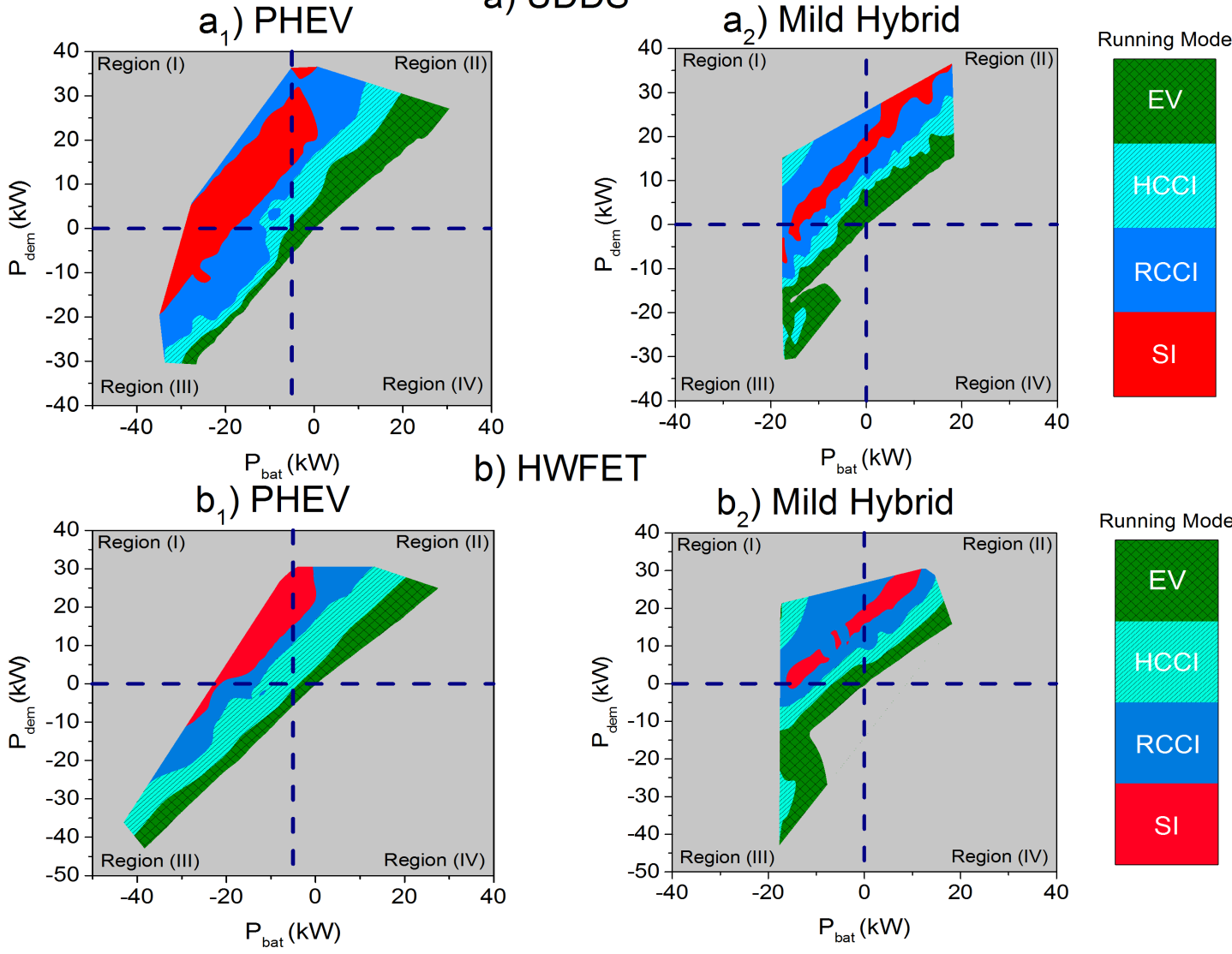

Running Mode

EV

He.

$\mathrm{RCCl}$

SI

Figure 6.7: Running modes of the LTC-SI HEV in different driving cycles and electrification levels.

In addition, Figure 6.7 aims to show the difference of engine usage for the PHEV and the mild hybrid. Note that the size of a region does not show the amount of time the powertrain has been operating in that region. For example, although the green zone in the region III of Figure 6.7 $a_{1}$ is narrower than that in Figure 6.7- $a_{2}$, the engine OFF time (i.e., EV mode) is higher compared to the mild hybrid. In the mild hybrid at high wheel power and high battery power in region II, the engine operates in the SI mode, whereas in the PHEV the engine does not run in the SI mode during the 
battery depletion. This is because, in mild hybrid during the high power demand, the engine has to operate in relatively high power region to compensate for the smaller battery power limit, while in PHEV, the battery can provide the required power due to its larger power limit compared to the mild hybrid. Thus, the control strategy uses the engine in SI mode to charge the battery faster in regions I and II.

Figures 6.8 and 6.9 show the engine optimal operating points for single-mode SI and multi-mode LTC-SI engines, respectively over the engine exhaust gas temperature map. All of the engine operating points meet the exhaust catalyst light-off temperature constraint (i.e., $T_{e x h} \geq 300{ }^{\circ} \mathrm{C}$ ). In Figure 6.9 it is shown even though the engine could operate in the HCCI mode in low engine torque region (i.e., $T_{\text {eng }}<50$ N.m) with lower BSFC, instead, it operates in RCCI mode to meet the catalyst lightoff temperature constraint. Thus, the powertrain ends up to compromise for lower emissions by losing fuel saving opportunities by running in RCCI mode compared to running in the HCCI mode. 
a) UDDS

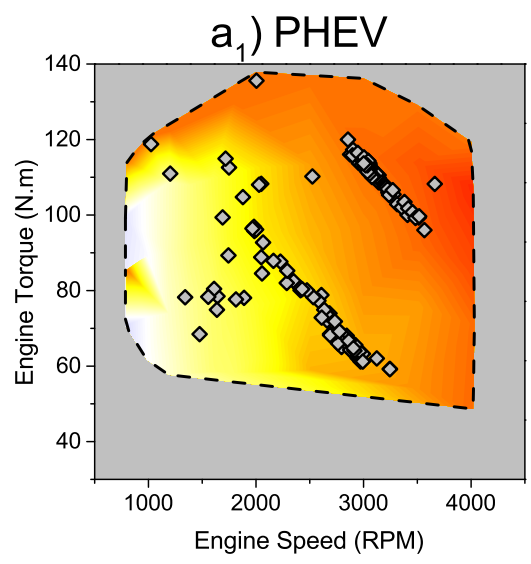

$a_{2}$ ) Mild Hybrid

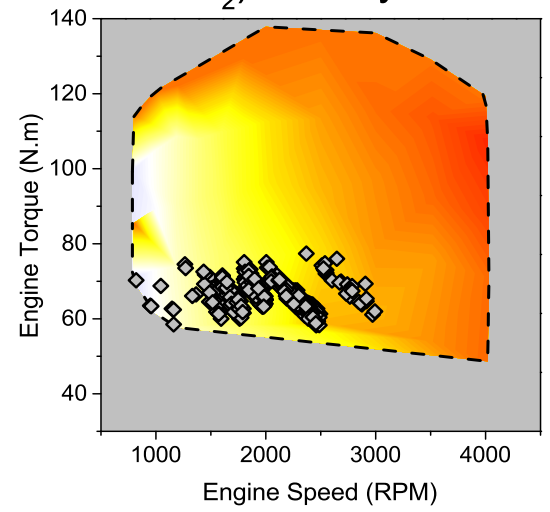

b) HWFET

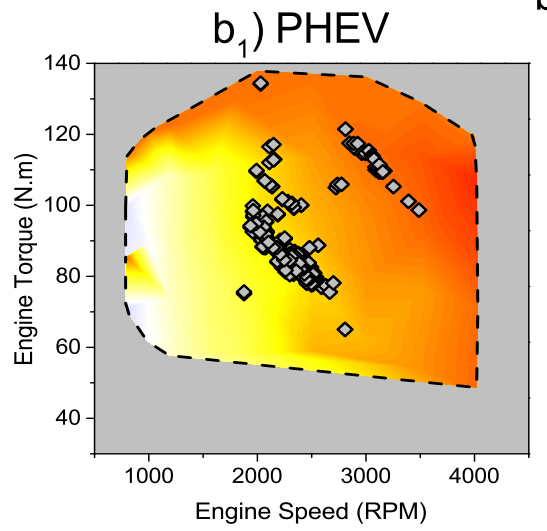

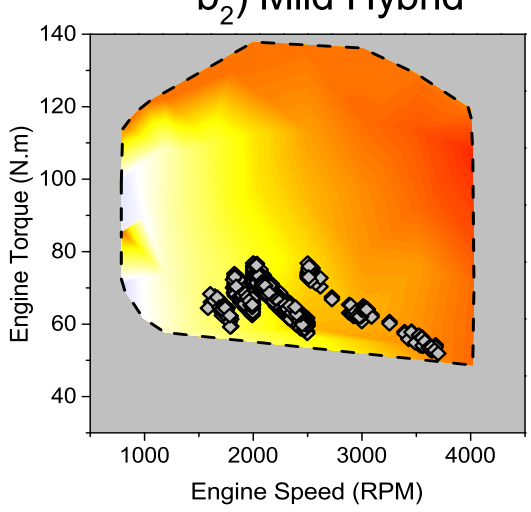

$b_{2}$ ) Mild Hybrid
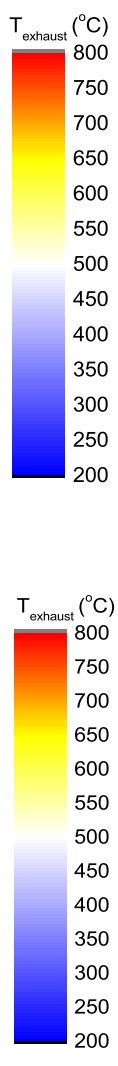

Figure 6.8: Engine operating points over the Single-mode engine exhaust gas temperature map for different electrification levels for a) UDDS and b) HWFET driving cycle. 

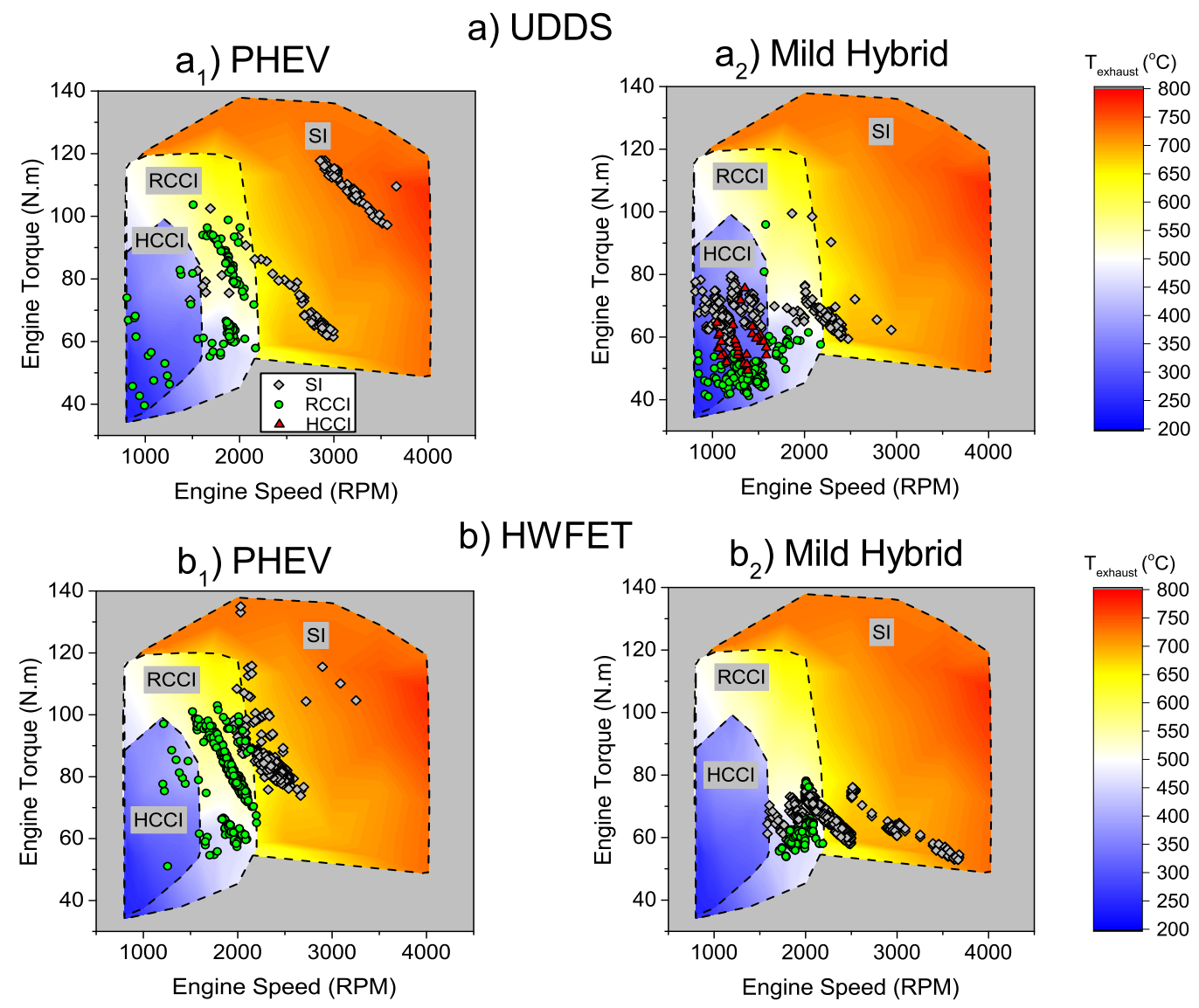

Figure 6.9: Engine operating points over the Multi-mode engine exhaust gas temperature map for different electrification levels for a) UDDS and b) HWFET driving cycle.

\subsection{Conclusions}

Fuel saving potential of utilizing a multi-mode LTC-SI engine in a parallel HEV in P2 architecture was investigated. The multi-mode engine included HCCI, RCCI, and SI modes. The PMP optimal energy management control technique was designed for the LTC-SI HEV powertrain and analyzed for different hybridization levels. The 
powertrain controller was designed to enable switching among different modes, while minimizing fuel consumption by including a penalty for transient engine operations. The exhaust gas temperature was included in the optimization framework to minimize the tailpipe emissions. Below are the main findings based on the optimization results in this chapter:

$\dagger$ The results for the UDDS driving cycle show the multi-mode LTC-SI engine offers up to $7.5 \%$ fuel saving over a single-mode SI engine in the parallel HEV. This improvement reduces to $0.7 \%$ for the HWFET driving cycle. This is because, in the highway driving cycle, the high power at wheels happen at high vehicle speeds, so the optimal control strategy needs to locate the engine operating points in the best BSFC region of the SI mode and run for a shorter time compared to the mild hybrid.

$\dagger$ The engine LTC running time increases from $7.6 \%$ to $20.5 \%$ by reducing the vehicle electrification level from PHEV to mild HEV, over the UDDS driving cycle. Among the LTC modes, the mid power RCCI was the dominant mode that had the most engine running time.

$\dagger$ The engine has less opportunity to operate in the low power region of the HCCI mode by considering the catalyst light-off temperature constraint. Instead, the optimal strategy chooses the RCCI mode as the engine operating mode with the higher exhaust gas temperature, compared to that in the HCCI mode. 
$\dagger$ In the parallel multi-mode LTC-SI powertrain, two ways for fuel savings include operating in the LTC mode or using electric torque assist offered by the e-motor. Fuel saving from these two ways are not additive. In strong hybridizations (e.g., PHEV) the optimizer commands to operate the engine in high power region of SI engine and turns off the engine for a longer time, while in mild hybrids, due to lack of sufficient electric power for charge-sustaining, the optimizer cannot locate the engine operating points in high power region, and, instead, the engine operating points shift to lower power regions which happen to be the LTC modes to save fuel.

$\dagger$ Compared to full electrified vehicles such as PHEVs, mild electrified vehicles such as mild HEVs are better suited to improve fuel economy in the multi-mode LTC-SI engine. 



\section{Chapter 7}

\section{Conclusion and Future Work}

Summary of the results and contributions of this dissertation are outlined in this chapter. In addition, recommendations for future work to extend the outcome from this dissertation are provided.

\subsection{Summary and Conclusion}

Integrating high efficiency combustion engines is a promising cost-effective solution to improve vehicles' fuel economy. LTC engines are a family of advanced combustion engines that provide higher or comparable BTE as CI engines, but they require less expensive aftertreatment systems for PM and NOx control. Even though the LTC 
engines benefit from higher BTE and less expensive NOx and PM aftretreatment systems, they have narrow operating range and require complex combustion control system which limit their usage in conventional powertrains. As the fleet merges to higher degree of powertrain electrification path, more opportunities for LTC engines will arise.

In this dissertation, an experimentally developed LTC engine was integrated with two hybridized powertrains including series and parallel for different hybridization levels. The ultimate fuel savings of the vehicle was investigated by developing optimal energy management control strategies including DP, MPC, and PMP. The fuel economy improvement of the LTC-HEV powertrains were compared with the SI/CI-HEV powertrains. To do so, an experimental electric powertrain test setup was developed and built at Michigan Technological University. The map-based models of the powertrain components were developed using the extracted test data.

In this study first single-mode LTC engines including HCCI and RCCI engines were studied in the SHEV and E-REV. Below are the summary of the findings for the single-mode LTC engines in the SHEV and E-REV:

$\dagger$ The results showed the HCCI engine offers significant potential for fuel saving in SHEV and E-REV architectures. In the UDDS driving cycle, integrating an HCCI engine with a series HEV powertrain can provide up to $18.9 \%$ higher fuel 
economy over a modern SI engine.

$\dagger$ The fuel economy improvement is found to be dependent on the EMC strategy applied. Among the three EMC strategies studied, the 3-mode DP offered the best performance with $17.1 \%$ higher fuel economy compared to the RBC for the HEV with the HCCI engine in the UDDS driving cycle.

$\dagger$ In the MPC strategy, by increasing the time horizon $(\mathrm{TH})$, the improvement in fuel economy increases and merges to the DP controller results. Changing the TH up to 70 seconds exhibited a strong effect on improving the fuel economy. But the rate of this improvement reduces and eventually becomes negligible once the MPC results merge to the DP results at $\mathrm{TH}=120$ seconds.

$\dagger$ Among the driving cycles (i.e., UDDS, NEDC, JC08), those that have higher average power (i.e., $P \geq 7.5 \mathrm{~kW}$ ) result in higher HCCI fuel saving. Thus, the HCCI engine operating in JC08 driving cycle offered $18.9 \%$ fuel economy improvement versus $16.2 \%$ in NEDC driving cycle in the E-REV mode.

$\dagger$ The simulation results showed the RCCI engine offers significant potential for fuel saving in SHEV architecture. In the combined driving cycle, integrating an RCCI engine with a SHEV powertrain provided up to $12.6 \%$ higher fuel economy over a modern SI engine, while the improvement over the CI-based HEV was $2.2 \%$ for the combined driving cycle (i.e., UDDS+HWFET+UDDS+US06+UDDS). 
$\dagger$ Increasing number of the engine operating points can increase the RCCI-HEV fuel economy (FE) improvement by $2.1 \%$ by utilizing more engine operating points.

$\dagger$ Among the four driving cycles studied, the driving cycle that has higher average power $(\bar{P})$ has higher HCCI/RCCI fuel saving. Thus, the HCCI/RCCI-HEV operating in US06 driving cycle $(\bar{P}=19.1 \mathrm{~kW})$ offered up to $14.2 \%$ fuel economy improvement versus $13.1 \%$ in UDDS driving cycle $(\bar{P}=5.2 \mathrm{~kW})$ in the SHEV. This was because the engine needs to run for a longer time to compensate for the higher power demand. This leads to more opportunity for the HCCI/RCCI engine to save more fuel in compared to the SI and CI engines.

$\dagger$ RCCI-SHEV operation with battery at low SOC in charge-sustaining mode resulted in slightly higher fuel economy improvement for the HCCI/RCCI engine in comparison to a higher initial SOC scenario. This is because the battery in low SOC region has larger energy loss which means the engine requires to run for a longer time to compensate for the higher battery losses. This provides more opportunity for the HCCI/RCCI engine to save more fuel in comparison to the SI and CI engines.

Next, fuel economy potential of utilizing a multi-mode LTC engine in a SHEV and parallel HEV architectures were investigated. To this end, the required experimental map-based models were developed using the LTC-SI electrified experimental setup. 
The PMP optimal control technique was employed and the powertrain controller was designed to enable switching among different modes, while minimizing fuel consumption by including penalty for transitions among different modes. The exhaust gas temperature was included in the optimization framework to consider the required exhaust aftertreatment efficiency for tailpipe emissions. Below, a summary of the findings for the multi-mode LTC engines as range extender in the SHEV architecture is listed:

$\dagger$ The simulation results for the UDDS cycle show the single-mode HCCI and RCCI engines offer up to $12.0 \%$ and $9.0 \%$ fuel economy improvement, respectively over a single-mode SI engine in the series E-REV. These improvements increase to $13.1 \%$ and $10.3 \%$ in the HWFET driving cycles for the HCCI and RCCI engines over the SI engine. This is due to longer engine ON time in the high power demand driving cycles. This provides more opportunity for the HCCI and RCCI engines to save more fuel compared to the SI engine.

$\dagger$ The HCCI and RCCI engines are more favorable from the NVH point of view since their operating points are at low engine speed region (i.e., $\omega_{\text {eng }} \leq 1500$ rpm), assuming combustion noise is controlled well in HCCI and RCCI engines. These engines have higher efficiency than the SI engine at relatively low engine speed, while at the low vehicle speeds (i.e., $V_{v e h} \leq 30 \mathrm{mph}$ ) the SI engine operates at high engine speeds (i.e., $\omega_{e n g} \geq 3000 \mathrm{rpm}$ ), which creates harsher noise. By 
applying the NVH constraint in selecting the engine operating points, the HCCI fuel economy improvement over the SI engine can go beyond the reported $12 \%$ in this work.

$\dagger$ Integrating a multi-mode LTC engine in the series E-REV offers $2 \%$ more fuel economy improvement compared the best fuel economy for the single-mode LTC engine in this study. This improvement depends on the type of driving cycle. High average power demand driving cycles (e.g., HWFET vs. UDDS) show higher fuel economy improvement.

$\dagger$ Reducing the mode-switching fuel penalty will increase the fuel economy of the series E-REV with the multi-mode LTC engine. Among the engine modes, HCCI is the dominant engine operating mode for the powertrain setup studied in this dissertation. If the fuel penalty to the RCCI mode decreases by 95 percent, the engine operates near to $45 \%$ of its run time in the RCCI mode.

Furthermore, fuel saving potential of utilizing a multi-mode LTC-SI engine in a parallel HEV in P2 architecture was investigated. The multi-mode engine includes HCCI, RCCI, and SI modes. Below are the main findings based on the optimization results in this study:

$\dagger$ The results for the UDDS cycle show the multi-mode LTC-SI engine offers up to $7.5 \%$ fuel saving over a single-mode SI engine in the parallel HEV. This 
improvement reduces to $0.7 \%$ for the HWFET driving cycle. This is because, in the highway driving cycles, the high wheel power happens at high vehicle speeds, so the optimal control strategy needs to locate the engine operating points in the best BSFC region of the SI mode and run for a shorter time compared to the mild hybrid.

$\dagger$ The engine LTC running time increases from $7.6 \%$ to $20.5 \%$ by reducing the vehicle electrification level from PHEV to mild HEV, over the UDDS driving cycle. Among the LTC modes, the mid power RCCI was the dominant mode that had the most engine running time.

$\dagger$ The engine has less opportunity to operate in the low power region $(P \leq 5.5$ $\mathrm{kW}$ ) of the HCCI mode by considering the exhaust catalyst light-off temperature constraint. Instead, the optimal strategy chooses the RCCI mode as the engine operating mode with the higher exhaust gas temperature, compared to that in the HCCI mode.

$\dagger$ In the parallel multi-mode LTC-SI powertrain, two ways for fuel savings include operating in the LTC mode or using electric torque assist offered by the e-motor. Fuel saving from these two ways are not additive. In strong hybridizations (e.g., PHEV) the optimizer commands to operate the engine in high power region of SI engine and turns off the engine for a longer time, while in mild hybrids, due to lack of sufficient electric power for charge-sustaining, the optimizer cannot locate the engine operating points in high power region, and, instead, the engine 
operating points shift to lower power regions which happen to be the LTC modes to save fuel.

$\dagger$ Compared to full electrified vehicles such as PHEVs, mild electrified vehicles such as mild hybrids are better suited to improve fuel economy in the multimode LTC-SI engine.

\subsection{Suggestions for Future Work}

Despite the promising results of integrating the LTC engines in hybridized electric powertrains, there is room for improvement and expanding the study. Here is the list of some research areas worthy of further investigation:

1. Analysis of cost versus fuel saving for the HEV powertrain using single-mode and multi-mode LTC engines versus SI engines in series and parallel HEV architectures.

2. Investigating bio-fueled LTC engines in hybridized electric powertrains. This will be of particular interest given upcoming US EPA regulations for $\mathrm{CO}_{2}$ emissions.

3. Studying the fuel economy improvement of hybridized multi-mode CI-RCCI engines in series HEV and parallel architectures over single-mode conventional 
engines.

4. Designing and implementing a fast real-time mode-based engine controller for mode-switching among different LTC and SI combustion modes. The controller is required to minimize the torque delay and misfire caused by switching among the engine combustion modes.

5. Real-time implementation of the multi-mode LTC-SI engines on the dynamometer to realize the promising fuel advantages of the powertrains proposed in this $\mathrm{PhD}$ dissertation.

6. The transmission design and optimal selection of gear ratios for the proposed hybrid electric powertrain require further investigations.

7. This study is focused on light duty vehicles. The proposed powertrain can be investigated for commercial and medium/heavy duty trucks.

8. A detailed emissions analysis of the hybridized multi-mode LTC-SI engine is required. In this work, the emission constraint is simplified by considering the catalyst light-off temperature in the optimization framework. In addition, engine combustion noise in LTC modes can be included as another optimization constraint.

The author believes that the research platform from this $\mathrm{PhD}$ thesis provides a proper starting ground to perform such future developments and investigations. 


\section{References}

[1] A. Solouk and M. Shahbakhti. Modeling and Energy Management of an HCCI based Powertrain for Series Hybrid and Extended Range Electric Vehicles. Int. Journal of Powertrains, 6(2). doi: 10.1504/IJPT.2017.10001761, 2017.

[2] A. Solouk and M. Shahbakhti. Energy Optimization and Fuel Economy Investigation of a Series Hybrid Electric Vehicle Integrated with Diesel/RCCI Engines. Energies, 9(12). doi: 10.3390/en9121020, 2016.

[3] A. Solouk, M. Shakiba, and M. Shahbakhti. Energy Analysis and Optimization of an Electrified Powertrain with a Multi-Mode High Efficiency Engine in Various Levels of Hybridizations. Submitted to Applied Energy, 49 pages, Dec. 2016.

[4] A. Solouk, J. Tripp, M. Shakiba, and M. Shahbakhti. Fuel Economy Assessment of an Extended Range Electric Vehicle with a Multi-Mode Low Temperature Combustion Engine. to be Submitted to Energy Conversions and Management, Feb. 2017. 
[5] SAFT Battery Company. High Power Lithium-ion VL7P cells. March 2005.

[6] UQM Technologies Inc., Powerphase 75 Traction System. http://www.neweagle.net/support/wiki/docs/Datasheets/UQM/PP75.pdf. Accessed on Dec. 2015.

[7] T.L. Nitz and D.UWE. Grebe. Voltec the Propulsion System for Chevrolet Volt and Opel Ampera. MTZ Magazine, 72, May 2011.

[8] S. Curran, R. Hanson, R. Wagner, and R. Reitz. Efficiency and Emissions Mapping of RCCI in a Light-Duty Diesel Engine. 2013. SAE Paper No. 2013-01-0289.

[9] J.K. Arora. Design of Real-Time Combustion Feedback System and Experimental Study of an RCCI Engine for Control. MSc. Thesis, Michigan Technological University, 2016.

[10] A. Solouk, M. Shakiba-herfeh, K. Kannan, H. Solmaz, P. Dice, M. Bidarvatan, N.N.T. Kondipati, and M. Shahbakhti. Fuel Economy Benefits of Integrating a Multi-Mode Low Temperature Combustion (LTC) Engine in a Series Extended Range Electric Powertrain. SAE Technical Paper No. 2016-01-2361, 2016.

[11] National Research Council. Cost, Effectiveness, and Deployment of Fuel Economy Technologies for Light-Duty Vehicles. The National Academies Press, page 1, 2015. doi:10.17226/21744. 
[12] European Commission Climate Action. https://ec.europa.eu/clima/policies. Accessed on Nov. 15, 2016.

[13] M. Berube. PHEVs - A Transition to the Future? SAE Range Extenders for Electric Vehicles Symposium, Knoxville, TN, USA, Nov. 2016.

[14] R. Sarkar. Balancing Regulations and Customer Expectations in Future Powertrain, Fuel, Lubricant Vehicle Systems. SAE International Powertrains, Fuels and Lubricants Meeting, Baltimore, MD, USA, Oct. 2016.

[15] Z. Gao, S.J. Curran, J.E. Parks, D.E. Smith, R.M. Wagner, C.S. Daw, K.D. Edwards, and J.F. Thomas. Drive Cycle Simulation of High Efficiency Combustions on Fuel Economy and Exhaust Properties in Light-Duty Vehicles. Applied Energy, 157:762-776, 2015.

[16] T.V. Johnson. Review of Diesel Emissions and Control. International Journal of Engine Research, 10(5):275-285, 2009.

[17] M. Shahbakhti and C.R. Koch. Characterizing the Cyclic Variability of Ignition Timing in a Homogeneous Charge Compression Ignition Engine Fuelled with n-heptane/iso-octane Blend Fuels. International Journal of Engine Research, 9(5):361-397, 2008.

[18] S. Curran, Z. Gao, and R. Wagner. Reactivity Controlled Compression Ignition Drive Cycle Emissions and Fuel Economy Estimations Using Vehicle Systems 
Simulations with E30 and ULSD. SAE International Journal of Engines, 7:902$912,2014$.

[19] R. Reitz and G. Duraisamy. Review of High Efficiency and Clean Reactivity Controlled Compression Ignition (RCCI) Combustion in Internal Combustion Engines. Progress in Energy and Combustion Science, 46(4):12-71, 2015.

[20] M. Shahbakhti and C.R. Koch. Physics Base Control Oriented Model for HCCI Combustion Timing. ASME Journal of Dynamic Systems, Measurment and Control, 132(2), 2010.

[21] M. Nazemi and M. Shahbakhti. Modeling and Analysis of Fuel Injection Parameters for Combustion and Performance of an RCCI Engine. Applied Energy, 165:135-150, 2016.

[22] A. Rezaei and J.B. Burl. Effects of time horizon on model predictive control for hybrid electric vehicles. IFAC-PapersOnLine, 48(15):252-256, 2015.

[23] L. Tribioli, M. Barbieri, R. Capata, E. Sciubba, E. Jannelli, and G. Bella. A Real Time Energy Management Strategy for Plug-In Hybrid Electric Vehicles Based on Optimal Control Theory. Energy Procedia, 45:949-958, 2014.

[24] A. Sciarretta, L. Serrao, P.C. Dewangan, P. Tona, E. Bergshoeff, C. Bordons, L. Charmpa, P. Elbert, L. Eriksson, and T. Hofman. A Control Benchmark on the Energy Management of a Plug-In Hybrid Electric Vehicle. Control Engineering Practice, 29:287-298, 2014. 
[25] D. Kum, H. Peng, and N.K. Bucknor. Supervisory Control of Parallel Hybrid Electric Vehicles for Fuel and Emission Reduction. Journal of Dynamic Systems, Measurment, and Control, 133(6), 2011.

[26] J.T.B.A. Kessels, M.W.T. Koot, P.P.J. Van den Bosch, and D.B. Kok. Online Energy Management for Hybrid Electric Vehicles. IEEE Transactions on Vehicular Technology, 57(6):3428-3440, 2008.

[27] A. Yonekawa, M. Ueno, O. Watanabe, and N. Ishikawa. Development of New Gasoline Engine for ACCORD Plug-in Hybrid. SAE Paper No. 2013-01-1738, 2013.

[28] N. Kawamoto, K. Naiki, T. Kawai, T. Shikida, and M. Tomatsuri. Development of New 1.8-Liter Engine for Hybrid Vehicles. SAE Paper No. 2009-01-1961, 2009.

[29] X. Hu, N. Murgovski, L. Johannesson, and B. Egardt. Energy Efficiency Analysis of a Series Plug-In Hybrid Electric Bus with Different Energy Management Strategies and Battery Sizes. Applied Energy, 111:1001-1009, 2013.

[30] S. Kermani, S. Delprat, T. Guerra, R. Trigui, and B. Jeanneret. Predictive Energy Management for Hybrid Vehicle. Control Engineering Practice, 20(4):408420, 2012.

[31] D.F. Opila, X. Wang, R. McGee, R.B. Gillespie, J. Cook, and J.W. Grizzle. An 
Energy Management Controller to Optimally Trade Off Fuel Economy and Drivability for Hybrid Vehicles. IEEE Transactions on Control Systems Technology, 20(6):1490-1506, 2012.

[32] A. Sciarretta, M. Back, and L. Guzzella. Optimal Control of Parallel Hybrid Electric Vehicles. IEEE Transactions on Control Systems Technology, 12(3):352$363,2004$.

[33] A. Brahma, Y. Guezennec, and G. Rizzoni. Optimal Energy Management in Series Hybrid Electric Vehicles. Proceeding of the American Control Conference, 1(6):60-64, 2000.

[34] B. Lawler, E. Ortiz-Soto, R. Gupta, H. Peng, and Z. Filipi. Hybrid Electric Vehicle Powertrain and Control Strategy Optimization to Maximize the Synergy with a Gasoline HCCI Engine. SAE Int. J. Engines, pages 1115-1126, 2011.

[35] A. Delorme, A. Rouseau, T. Wallner, E. Ortiz-Soto, A. Babajimopolous, and D. Assanis. Evaluation of Homogeneous Charge Compression Ignition (HCCI) Engine Fuel Savings for Various Electric Drive Powertrains. 25th World Battery, Hybrid and Fuel Cell Electric Vehicle Symposium \& Exhibition, 2010.

[36] S. Nüesch and A.G. Stefanopoulou. Multimode combustion in a mild hybrid electric vehicle. Part 1: Supervisory control. Control Engineering Practice, 57:99$110,2016$.

[37] R. Hanson, S. Spannbauer, C. Gross, R.D. Reitz, S. Curran, J. Storey, and 
S. Huff. Highway Fuel Economy Testing of an RCCI Series Hybrid Vehicle. SAE Paper No. 2015-01-0837, 2015.

[38] S.C. Davis, S.W. Diegel, and R.G. Boundy. Transportation Energy Databook. Oak Ridge National Lab, TN, Edition 31, Ch. 2, July 2012.

[39] Annual Energy Outlook 2013 with projections to 2040. U.S. Energy Information Administration (EIA), (DOE/EIA-0383(2013)), April 2013.

[40] T. McCarthy. Systems Approach to Making Optimal Powertrain Technology Choices - A Global OEM's Perspective. ASME ICE Fall Tech. Conference, 2013.

[41] S. Saxena, N. Shah, I. Bedoya, and A. Phadke. Understanding Optimal Engine Operating Strategies for Gasoline-Fueled HCCI Engines Using Crank-Angle Resolved Exergy Analysis. Applied Energy, 114:pages 155-163, 2014.

[42] S. Kermani, S. Delprat, T. Guerra, R. Trigui, and B. Jeanneret. Predictive Energy Management for Hybrid Vehicle. Control Engineering Practice, 20(4):408420, 2012.

[43] D.F. Opila, X. Wang, R. McGee, R.B. Gillespie, J. Cook, and J.W. Grizzle. An Energy Management Controller to Optimally Trade Off Fuel Economy and Drivability for Hybrid Vehicles. IEEE Transactions on Control Systems Technology, 20(6):1490-1506, 2012.

[44] R. Johri, A. Salvi, and Z. Filipi. Optimal Energy Management For a Hybrid 198 
Vehicle Using Neuro-Dynamic Programming to Consider Transient Engine Operation. ASME DSCC Paper No. 2011-6138, 2011.

[45] N. Jalil and M. Salman. A Rule-Based Energy Management Strategy for a Series Hybrid Vehicle. Proceedings of American Control Conference, 1:689-693, 1997.

[46] I.J. Albert, E. Kahrimanovic, and A. Emadi. Diesel Sport Utility Vehicles with Hybrid Electric Drive Trains. IEEE Transactions on Vehicular Technology, 53(4):1247-1256, 2004.

[47] A. Sciarretta, M. Back, and L. Guzzella. Optimal Control of Parallel Hybrid Electric Vehicles. IEEE Transactions on Control Systems Technology, 12(3):352363, 2004.

[48] C. Lin, H. Peng, and J.W. Grizzle. A Stochastic Control Strategy for Hybrid Electric Vehicles. Proceeding of the American Control Conference, 5:4710-4715, 2004.

[49] A. Brahma, Y. Guezennec, and G. Rizzoni. Optimal Energy Management in Series Hybrid Electric Vehicles. Proceeding of the American Control Conference, 1(6):60-64, 2000 .

[50] A. Brahma, B. Glenn, Y. Guezennec, T. Miller, G. Rizzoni, and G. Washington. Modeling, Performance Analysis and Control Design of a Hybrid Sport-Utility Vehicle. Proceeding of the IEEE Int. Conference on Control Application, 1:448453, 1999. 
[51] L. Tribioli, M. Barbieri, and et al. A Real Time Energy Management Strategy for Plug-In Hybrid Electric Vehicles Based on Optimal Control Theory. Energy Procedia, 45:949-958, 2014.

[52] A. Sciarretta, L. Serrao, P.C. Dewangan, P. Tona, E. Bergshoeff, C. Bordons, L. Charmpa, P. Elbert, L. Eriksson, and T. Hofman. A Control Benchmark on The Energy Management of a Plug-In Hybrid Electric Vehicle. Control Engineering Practice, 29:287-298, 2014.

[53] M. Shams-Zahraei, A. Kouzani, S. Kutter, and B. Bäker. Integrated Thermal and Energy Management of Plug-In Hybrid Electric Vehicles. Journal of Power Sources, 216:237-248, 2012.

[54] J.T.B.A. Kessels, M.W.T. Koot, P.P.J. Van den Bosch, and D.B. Kok. Online Energy Management for Hybrid Electric Vehicles. IEEE Transactions on Vehicular Technology, 57(6):3428-3440, 2008.

[55] L. Serrao, S. Onori, and G. Rizzoni. A Comparative Analysis of Energy Management Strategies for Hybrid Electric Vehicles. ASME Journal of Dynamic Systems, Measurement, and Control, 133(3), 2000.

[56] S.G. Li, S.M. Sharkh, F.C. Walsh, and C.N. Zhang. Energy and Battery Management of a Plug-In Series Hybrid Electric Vehicle Using Fuzzy Logic. IEEE Transactions on Vehicular Technology, 60(8):3571-3585, 2011.

[57] D. Kum, H. Peng, and N.K. Bucknor. Supervisory Control of Parallel Hybrid 
Electric Vehicles for Fuel and Emission Reduction. Journal of Dynamic Systems, Measurment, and Control, 133(6), 2011.

[58] M. Back, M. Simons, F. Kirschaum, and V. Krebs. Predictive control of drivetrains. In Proc. of 15th IFAC World Congress, Barcelona, Spain, 2002.

[59] K. Ahn, J. Whitefoot, A. Babajimopoulos, E. Ortiz-Soto, and P. Papalambros. Homogenous Charge Compression Ignition Technology Implemented in a Hybrid Electric Vehicle: System Optimal Design and Benefit Analysis for a Power-Split Architecture. Proceedings of the Institution of Mechanical Engineers, Part D: Journal of Automobile Engineering, 227(1):87-98, 2012.

[60] M. Shahbakhti and C.R. Koch. Characterizing the Cyclic Variability of Ignition Timing in an HCCI Engine Fueled with n-Heptane/iso-Octane Blend Fuels. Int. Journal of Engine Research, 9(5):361-397, 2008.

[61] M. Bidarvatan and M. Shahbakhti. Two-input two-output control of blended fuel hoci engines. SAE Paper No. 2013-01-1663, 2013.

[62] O. Tremblay, L. Dessaint, and A. Dekkiche. A Generic Battery Model for the Dynamic Simulation of Hybrid Electric Vehicles. IEEE Conference on Vehicle Power and Propulsion, pages 284-289, Arlington, TX, Sept. 2007.

[63] G. Byeon, T. Yoon, S. Oh, and G. Jang. Energy Management Strategy of the DC Distribution System in Building Using the EV Service Model. IEEE Transaction on Power Electronics, 28(4):1544-1554, 2013. 
[64] C. Rosenkranz. Deep Cycle Batteries for Plug-in Hybrid Application. In Proc. Plug-In Hybrid Veh. Workshop, 20th Int. Elect. Veh. Symp, Long Beach, CA, 2003.

[65] 2010 Honda Insight Hybrid Electric Vehicle Testing Report. http://avt.inel.gov/pdf/hev/fact2010hondainsight.pdf/. Idaho National Laboratory. Accessed on Dec 2015.

[66] F. Salmasi. Control Strategies for Hybrid Electric Vehicles: Evolution, Classification, Comparison, and Future Trends. IEEE Transaction on Vehicular Technology, 56(5):2393-2404, 2007.

[67] F. Lewis, D.L. Vrabie, and V. Syrmos. Optimal Control. John Wiley and Sons Inc., Chapter 5, 3rd Edition, 2012.

[68] G. Rizzoni, L. Guzzella, and B.M. Baumann. Unified Modeling of Hybrid Electric Vehicle Drivetrains. IEEE Transactions on Mechatronics, 4(3):246-257, 1999.

[69] A. Sciarretta and L. Guzzella. Control of Hybrid Electric Vehicles, Optimal Energy Management Strategies. IEEE Control Systems Magazine, 27(2):60-70, 2007.

[70] A. Khajepour, S. Fallah, and A. Goodarzi. Electric And Hybrid Vehicles, Technologies, Modeling and Control: A Mechatronic Approach. Wiley. Edition 1, Chapter 5, 2014. 
[71] A.A. Malikopoulos. Supervisory Power Management Control Algorithms for Hybrid Electric Vehicles: A survey. IEEE Transaction on Intelligent Transportation, 15(5):12-71, 2014.

[72] S.C. Davis, S.W. Diegel, and R.G. Boundy. EPA and NHTSA Set Standards to Reduce Greenhouse Gases and Improve Fuel Economy for Model Years 2017-2025 Cars and Light Trucks. U.S. Environmental Protection Agency, August 2012.

[73] European Commission Climate Action. http://ec.europa.eu/clima/policies. Accessed on May 2016.

[74] J. Li, W.M. Yang, H. An, and D. Zhao. Effects of Fuel Ratio and Injection Timing on Gasoline/Biodiesel Fueled RCCI Engine: A Modeling Study. Applied Energy, 155:59-67, 2015.

[75] B.C. Chen, Y.Y. Wu, and H.C. Tsai. Design and Analysis of Power Management Strategy for Range Extended Electric Vehicle Using Dynamic Programming. Applied Energy, 113(7):1764-1774, 2014.

[76] X. Wang, H. He, F. Sun, and J. Zhang. Application Study on the Dynamic Programming Algorithm for Energy Management of Plug-in Hybrid Electric Vehicles. Energies, 8(4):3225-3244, 2015.

[77] S. Zhang and R. Xiong. Adaptive Energy Management of a Plug-In Hybrid Electric Vehicle Based on Driving Pattern Recognition and Dynamic Programming. Applied Energy, 155:68-78, 2015. 
[78] W. Shabbir and S.A. Evangelou. Real-Time Control Strategy to Maximize Hybrid Electric Vehicle Powertrain Efficiency. Applied Energy, 135:512-522, 2014.

[79] J. Fan, J. Zhang, and T. Shen. Map-Based Power-Split Strategy Design with Predictive Performance Optimization for Parallel Hybrid Electric Vehicles. Energies, 8(9):9946-9968, 2015.

[80] X. Hu, N. Murgovski, L. Johannesson, and B. Egardt. Energy Efficiency Analysis of a Series Plug-In Hybrid Electric Bus with Different Energy Management Strategies and Battery Sizes. Applied Energy, 111:1001-1009, 2013.

[81] A. T. Zaremba, C. Soto, M. Shakiba-herfeh, and M. Jennings. Power management of hybrid electric vehicles based on pareto optimal maps. SAE International Journal of Alternative Powertrains, 3(1):56-63, 2014.

[82] V.Y. Prikhodko, S. Curran, J.E. Parks, and R. Wagner. Effectiveness of Diesel Oxidation Catalyst in Reducing $\mathrm{HC}$ and $\mathrm{CO}$ Emissions from Reactivity Controlled Compression Ignition. SAE Int. J. Fuels Lubr., 6(1):329-335, 2014.

[83] E. Ansari, K. Poorghasemi, B. K. Irdmousa, M. Shahbakhti, and J. Naber. Efficiency and Emissions Mapping of a Light Duty Diesel-Natural Gas Engine Operating in Conventional Diesel and RCCI Modes. SAE Technical Paper No. 2016-01-2309, 2016. 
[84] J.L. Torres, R. Gonzalez, A. Gimenez, and J. Lopez. Energy Management Strategy for Plug-In Hybrid Electric Vehicles. A Comparative Study. Applied Energy, 113:816-824, 2014.

[85] V. Thakkar. Modeling and Experimental Setup of an HCCI Eengine. MSc. Thesis, Michigan Technological University, 2014.

[86] D. Kothari. Experimental Setup and Controller Design for an HCCI Engine. MSc. Thesis, Michigan Technological University, 2014.

[87] H.A. Saigaonkar. An Investigation of Variable Valve Timing Effects on HCCI Engine. MSc. Thesis, Michigan Technological University, 2015.

[88] K. Kannan. An Experimental Investigation of Low Temperature Combustion Regimes in a Light Duty Engine. MSc. Thesis, Michigan Technological University, 2016.

[89] M. Shahbakhti, A. Ghazimirsaied, and C.R. Koch. Experimental Study of Exhaust Temperature Variation in a Homogeneous Charge Compression Ignition Engine. Proceedings of the Institution of Mechanical Engineers, Part D: Journal of Automobile Engineering, 224(9):1177-1197, 2010.

[90] N. Kim, S. Cha, and H. Peng. Optimal Control of Hybrid Electric Vehicles Based on Pontryagin's Minimum Principle. IEEE Transactions on Control Systems Technology, 19(5):1279-1287, 2011. 
[91] S. P. Nüesch. Analysis and Control of Multimode Combustion Switching Sequence. PhD Thesis, The University of Michigan, 2015.

[92] M. Ehsani, Y. Gao, and A. Emadi. Modern Electric, Hybrid Electric, and Fuel Cell Vehicles. CRC Press. Chapter 7, Edition 2, 2010.

[93] A. Solouk, M. Shakiba-herfeh, and M. Shahbakhti. Analysis and Control of a Torque Blended Hybrid Electric Powertrain with a Multi-Mode LTC-SI Engine. SAE Int. J. of Alternative Powertrains, 15 pages, 6(1):2017, doi:10.4271/201701-1153, 2017.

[94] A. Solouk, M. Shakiba-herfeh, and M. Shahbakhti. Analysis and Control of a Torque Blended Hybrid Electric Powertrain with a Multi-Mode LTC-SI Engine. SAE Technical Paper No. 2017-01-1153, $201 \%$.

[95] A. Solouk, M. Shahbakhti, and M.J. Mahjoub. Energy Management and Control of a Hybrid Electric Vehicle with an Integrated Low Temperature Combustion (LTC) Engine. ASME Dynamic Systems and Control Conference, 22-24 Oct. 2014, San Antonio, TX, USA. 


\section{Appendix A}

\section{Series HEV Components Sizing}

\section{A.1 Series HEV Components Sizing}

This appendix presents sizing of the e-motor and the battery pack for the series HEV model used in chapters 2 and 3 of this dissertation.

\section{A.1.1 Power Rating Design of Traction E-motor}

The power rating of the E-motor in a series HEV is determined by the vehicle accel-

eration performance requirement and E-motor characteristics [92]. Equation (A.1) is used to estimate the power rating of the E-motor to provide a desired acceleration 
performance,

$$
P_{t}=\frac{\delta M}{2 t_{a}}\left(V_{f}^{2}+V_{b}^{2}\right)+\frac{2}{3} M g f_{r} V_{f}+\frac{1}{5} \rho C_{d} A V_{f}^{3}
$$

where $M$ is the total vehicle mass in $\mathrm{kg}, t_{a}$ is the acceleration time in seconds, $V_{b}$ is the vehicle speed in $\mathrm{m} / \mathrm{sec}$ corresponding to the E-motor base speed. $V_{f}$ is the final vehicle speed after acceleration. As previously mentioned in chapter 2 , $t_{a}=12.2$ sec and the final vehicle speed $\left(V_{f}\right)=60 \mathrm{mph}$ are selected. The $V_{b}$ depends on the E-motor characteristics. Speed ratio $(x)$ is defined as E-motor maximum speed to the base speed $\left(x=\frac{V_{\max }}{V_{b}}\right)$. Figure A.1 shows the E-motor power rating versus the speed ratio in this appendix.

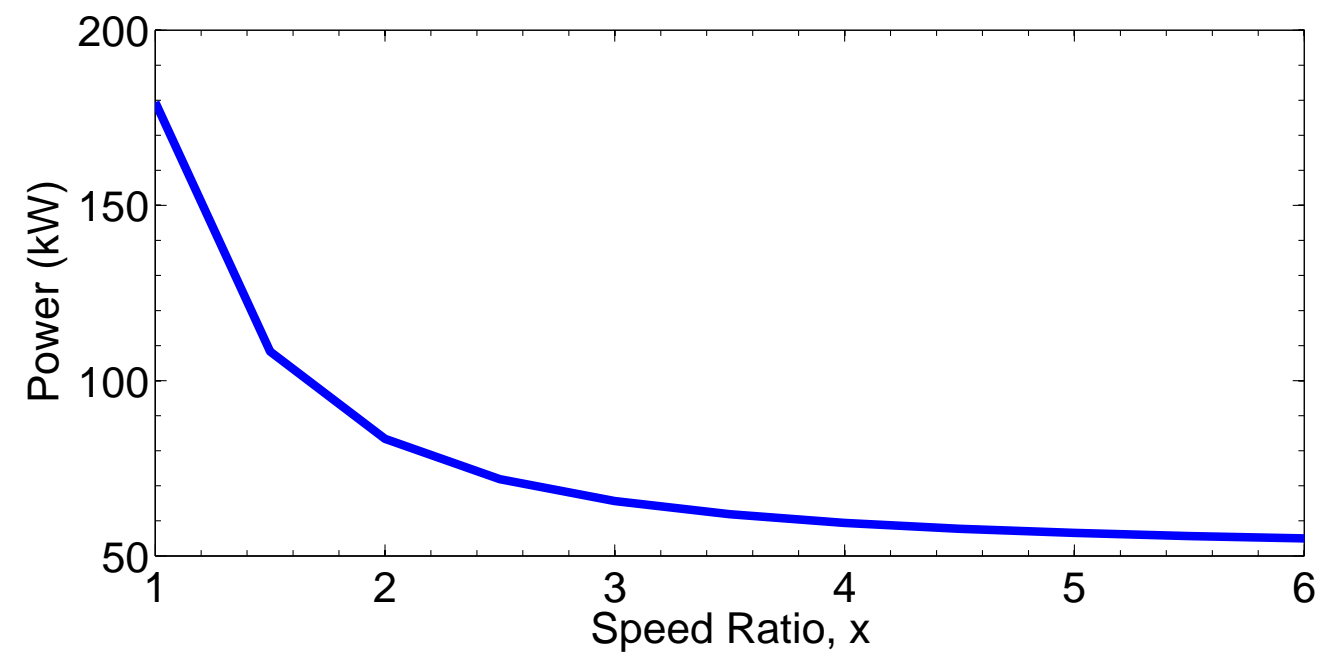

Figure A.1: Required power from the traction E-motor for the vehicle acceleration. 
The required traction power for hill climbing conditions is found by:

$$
P_{\text {grade }}=\left(M g f_{r} \cos \alpha+\frac{1}{2} \rho_{a} C_{d} A V^{2}+M g \sin \alpha\right) \frac{V}{1000}
$$

where $\alpha$ is the ground slope angle and $V$ is the vehicle speed. $\alpha$ is selected based on the gradeability constraints for a common mid-size vehicle from [65].

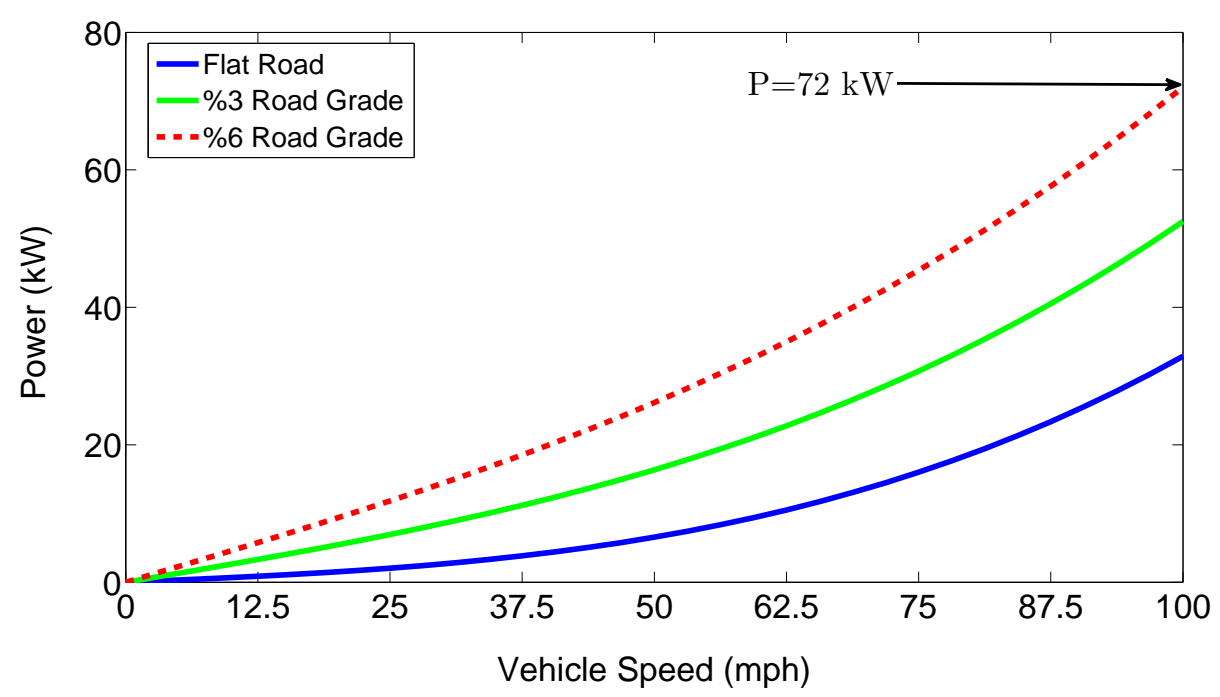

Figure A.2: Required power from the traction E-motor for the vehicle gradability.

Figure A.2 shows the required power rating for the traction E-motor to meet the gradeability constraint. It can be seen from Figure A.2 that $72 \mathrm{~kW}$ traction power is needed for the vehicle to meet the gradeability constraint. During acceleration, the E-motor power rating also depends on the E-motor speed ratio $(x)$. The $x$ value is a characteristic feature of the E-motors and this value varies in different E-motors. In this appendix, six different E-motors available in the HEV market are examined, as 
shown in Figure A.3.

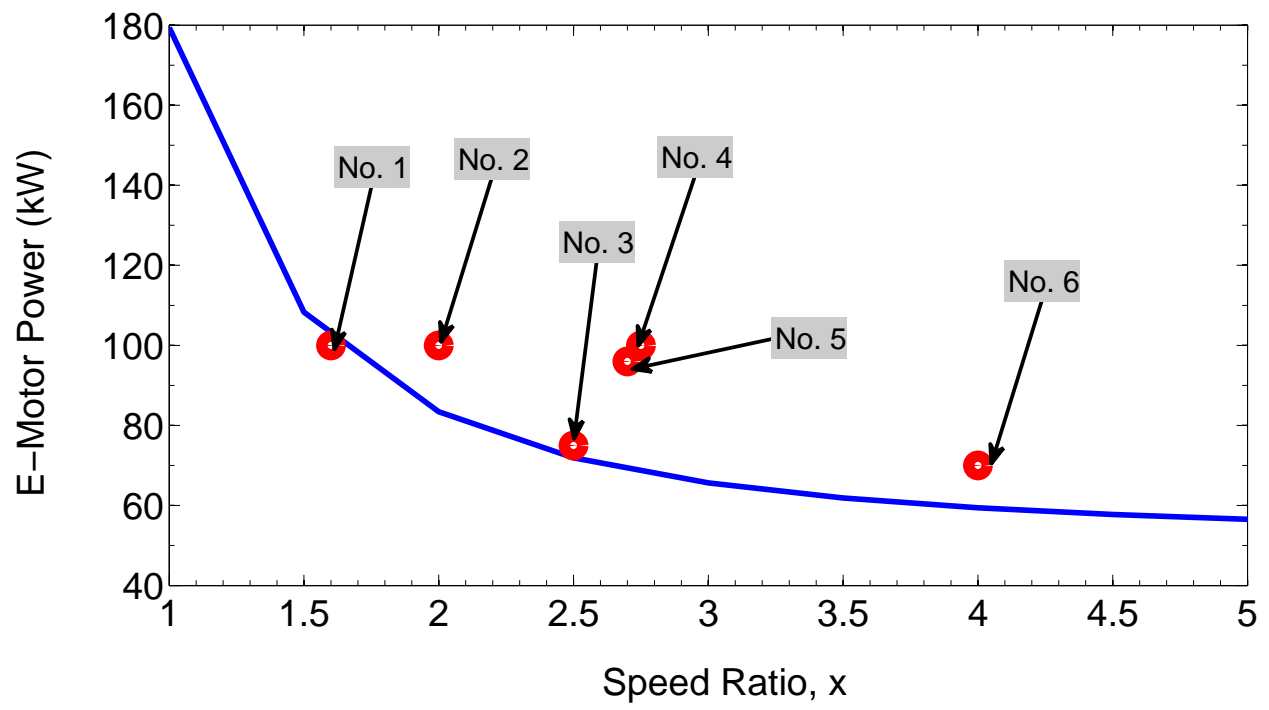

Figure A.3: Different E-motors studied in this appendix.

An E-motor with power close to the solid line in Figure A.3 is desirable. It can be seen the E-motor \#1 and \#3 are very close to the solid line. The rated power of E-motor \#1 and \#3 are $108 \mathrm{~kW}$ and $75 \mathrm{~kW}$, respectively. E-motor \#3 is better as it needs a smaller battery pack. Therefore, E-motor \#3 (i.e., UQM PowerPhase 75) is selected since it can meet both the acceleration performance and gradeability power requirement, while requiring a minimal battery pack.

\section{A.1.2 Engine}

Figure A.4 shows the required power from the engine to maintain the vehicle at constant speeds. The simulation results show that the engine needs to produce at 
least $15 \mathrm{~kW}$ to keep the vehicle in $70 \mathrm{mph}$ constant vehicle speed.

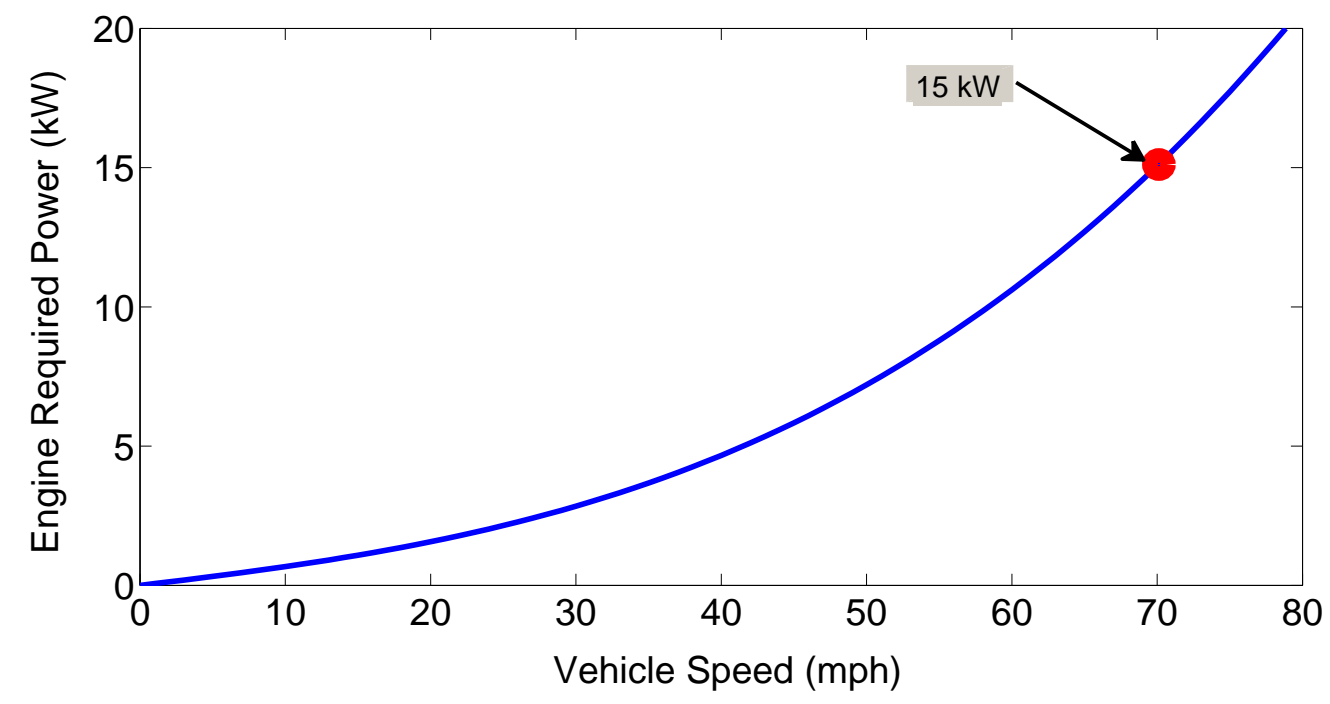

Figure A.4: Engine Required Power Rating at Constant Vehicle Speeds.

\section{A.1.3 Battery}

The battery power and energy capacity should be selected to meet required power to the traction E-motor and also store sufficient energy to avoid failure in power delivery due to deep discharging.

\section{A.1.3.1 Power Capacity}

The total power of the battery and engine/generator should be equal or greater than the rated maximum power of the E-motor $\left(P_{m, \max }\right)$ : 


$$
P_{b} \geq \frac{P_{m, \max }}{\eta_{\text {motoring }}}-P_{\text {gen }}
$$

where $P_{b}$ is the maximum rated power of the battery, $\eta_{\text {motoring }}$ is the efficiency of the E-motor, and $P_{g e n}$ is the power of the engine/generator system. In this appendix, by considering the average E-motor efficiency of $85 \%$ and generator efficiency of $95 \%$, the battery pack rated power is determined to be greater than $63 \mathrm{~kW}$.

\section{A.1.3.2 Energy Capacity}

The energy capacity determines the time duration that the battery can provide the required power. This capacity highly depends on the driving cycle. As a general rule, a mid-size sedan requires averagely requires $300 \mathrm{Wh}$ energy per mile for a highway driving cycle [64]. In this appendix, the battery energy capacity is chosen to provide 40 miles AER for the E-REV. The $\triangle S O C=0.65$ similar to 2011 Chevy Volt E-REV is selected. Thus, the battery total energy capacity needs to be $18.5 \mathrm{kWh}$ in order to provide the AER of the vehicle up to 40 miles.

Based on the power capacity and energy capacity, the number of required battery cells is determined. Equations (A.4) to (A.6) are used to calculate battery model 
parameters:

$$
\begin{gathered}
E_{0}=E_{\text {full }}+K+R i-A \\
A=E_{\text {full }}-E_{\exp } \\
B=\frac{3}{Q_{\exp }}
\end{gathered}
$$

where $E_{0}$ is battery constant voltage, $R$ is battery internal resistance, $i$ is battery discharge current, $A$ is voltage drop amplitude during the battery exponential discharge zone, and $K$ is polarization voltage [63] and is deduced from Equation (2.1); $E_{\text {full }}$ is fully charged voltage, $E_{\text {exp }}$ is voltage at the end of exponential discharge curve zone, $B$ is the time constant inverse for the exponential discharge curve zone, and $Q$ is battery nominal capacity. Table A.1 lists model parameters for the battery pack selected for this appendix.

\section{Table A.1}

Parameters of the battery (i.e., LV30P SAFT) selected in this appendix.

\begin{tabular}{lc}
\hline \hline Parameters & Values \\
\hline$E_{\text {full }}(\mathrm{V})$ & 414 \\
$E_{\text {exp }}(\mathrm{V})$ & 395 \\
$E_{\text {nom }}(\mathrm{V})$ & 354 \\
$Q(\mathrm{Ah})$ & 30 \\
$Q_{\text {exp }}(\mathrm{Ah})$ & 3.15 \\
$Q_{\text {nom }}(\mathrm{Ah})$ & 26.6 \\
$A(\mathrm{~V})$ & 103.6 \\
$B(\mathrm{Ah})^{-1}$ & 0.96 \\
$K(\mathrm{~V})$ & 5.2 \\
$R_{\text {eq }}(\Omega)$ & 0.0514 \\
\hline \hline
\end{tabular}





\section{Appendix B}

\section{Electric Motor Experimental Setup}

An experimental electric motor setup was designed and built as part of this thesis. This was briefly explained in Chapter 4, Here, details for the electric motor experimental setup are provided.

\section{B.1 Control Strategy Developed to Run the Sys- tem}

The system which includes the battery, RMS inverter and PM AC Motor are connected through HV cables and communication interface between the sub-systems, a low voltage CAN communication is used as shown in Figure 4.4. The control strategy 
for the battery, inverter, and motor is developed in Simulink environment. The CAN bus is connected across the system and also to the MABx. The developed Simulink control model generated code is flashed in MABx to implement the strategies. The CAN communication is set up with a default rate of $250 \mathrm{~K}$ baud rate. The available baud rates include $125 \mathrm{~K}, 250 \mathrm{~K}, 500 \mathrm{~K}$, and $1 \mathrm{M}$. The terminator resistor is available and is connected to the junction box. Figure B.1 depicts that the model including three main sub blocks as sensors, controller, and actuator blocks. Important parameters of battery and inverter including the voltages, current, temperature, battery contactor status, and inverter enable status are received in CAN communication (i.e., sensor block) and are sent to the controller block. The controller program, developed in the StateFlow (see Figure B.2), manages the required actions. These actions include enabling the battery+HVBox, and inverter to send the torque command to the dyno.

For enabling the battery, the following procedure is programmed on dSPACE:

1. Send the battery wake up signal to activate the battery BMS.

2. Close the battery negative contactor.

3. Close the battery positive contactor and wait for 3 seconds.

4. Close the HV box pre-charge relay and wait for 3 seconds.

5. Measure the voltage difference between battery output and the inverter input 


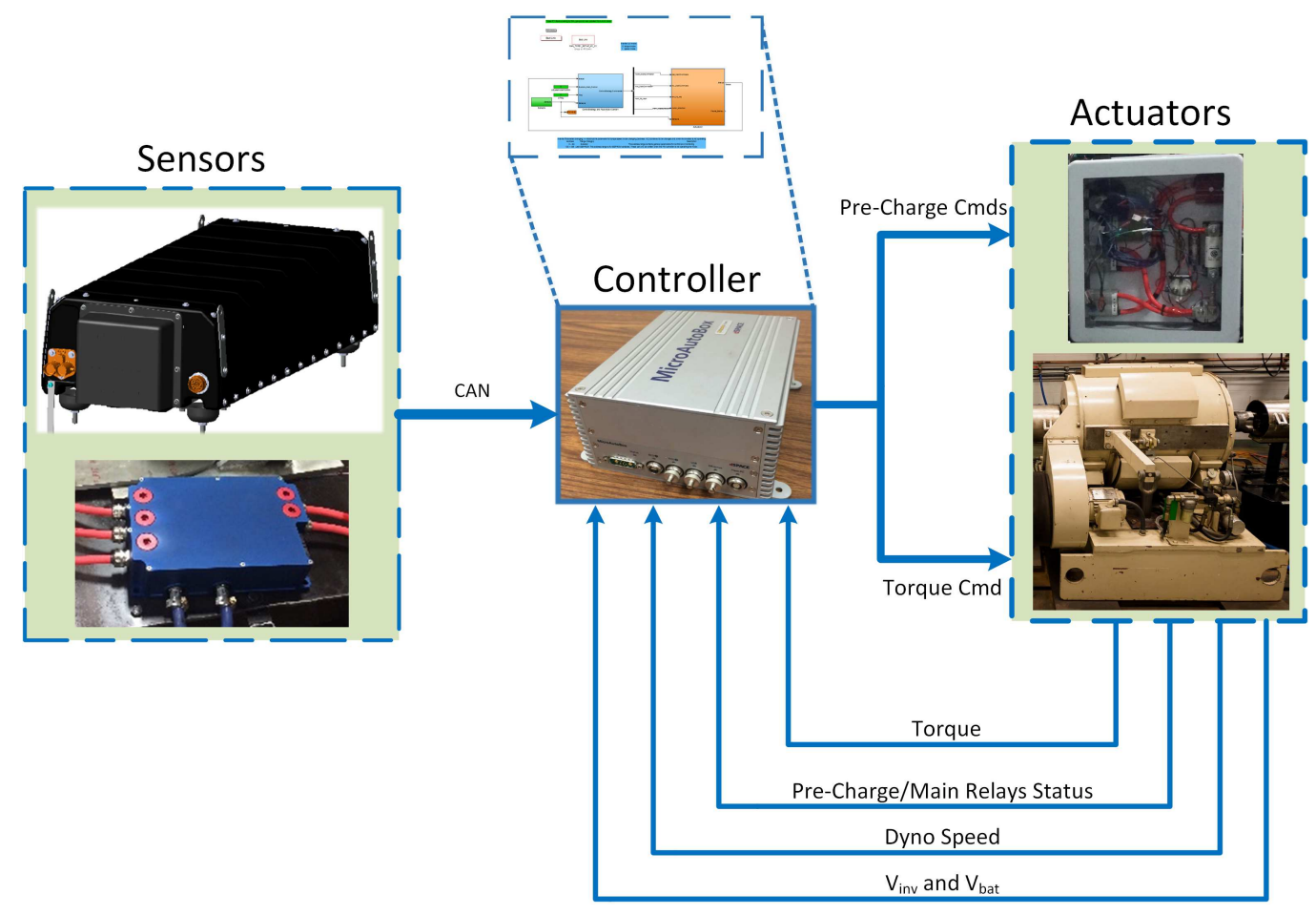

Figure B.1: Developed program for handling the setup startup/run and model-based control of the e-motor setup.

terminal. If the difference is less than 1 volt, the battery enabling process is completed, otherwise, an error will be reported by the software and the battery will be turned off.

Moreover, after the inverter could sense the HV provided by the battery on the input terminals, then the inverter enabling procedure is programmed as:

1. Check for the inverter and e-motor temperature.

2. Confirm that the motor shaft speed is zero.

3. Deactivate the inverter lockout signal. 
(a)

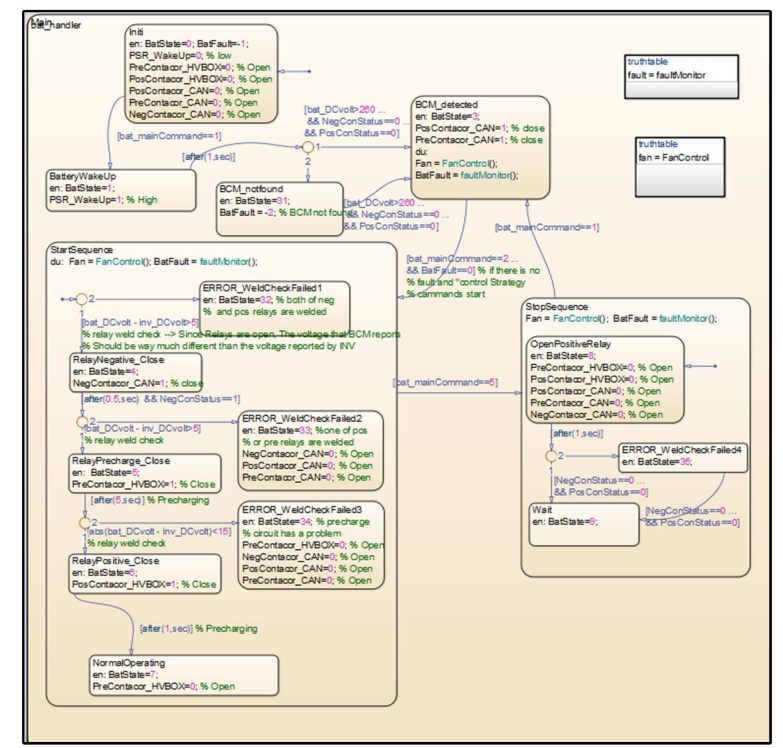

(b)

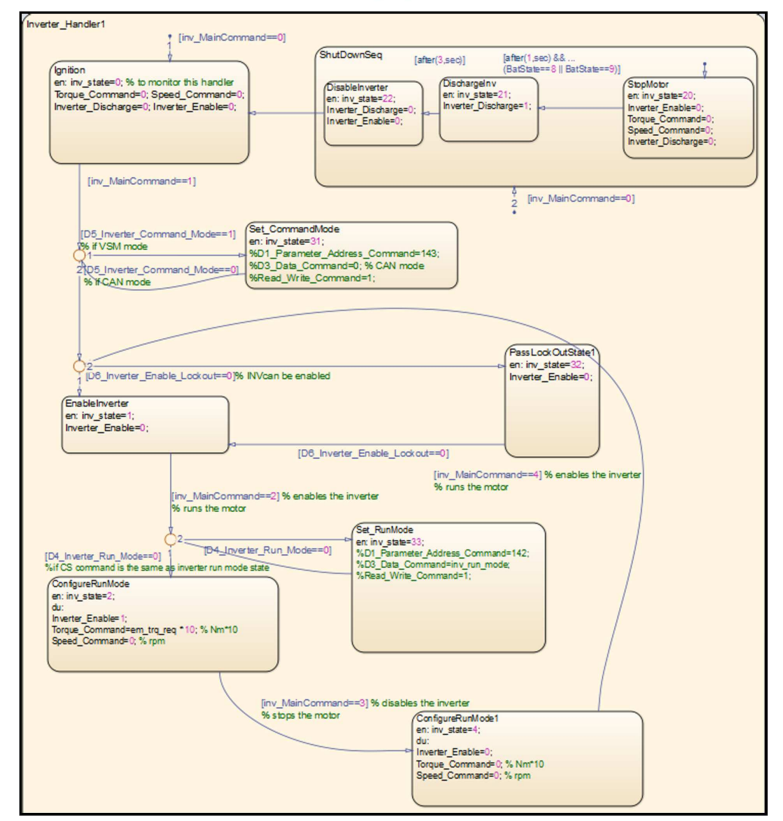

Figure B.2: Snapshot of the developed StateFlow handler programs for (a) battery (b) inverter.

4. The inverter is enabled and is ready to receive the torque command. 


\section{B.2 Battery}

This section gives the details on the LGChem battery used for this dissertation at the Energy Mechatronics Laboratory at APSRC, Michigan Technological University.

The battery main components include:

- Main service disconnect (MSD): The MSD serves as the important safety feature, used to isolate the battery from unwanted terminal voltage rendering while not in use. It is a safety kill switch, which when removed from the Battery, makes it involuntary to use. MSD should be kept locked in a safe and secure place and should not be transferred to people without prior approval from the person in charge.

- High voltage supply connectors: The battery power out cable is used to connect to the inverter which powers alternating current to the e-Motor. The two leads from the Battery Connection is provided via an Anderson connector. This female Anderson connector out is the terminal power out, which is to be connected to the Inverter.

- Battery management system (BMS) unit: BMS unit, which is located at the rear end of the battery, provides the necessary power supply to the inbuilt BMS, controls the fan switches, contactor positions and also deciphers communication channels. A $12 \mathrm{~V}$ power supply is to be connected across the terminals of the BMS. There will 
be 3 different positive terminals one each for fan power supply, vehicle CAN bus and the BMS unit. All of them are connected together and connected to the $12 \mathrm{~V}$ of the power supply. There is a common ground for all which is connected to the negative terminal of the power supply.

For communicating with the battery through CAN, first required wires and connectors are built. Then, a battery handler program is developed in StateFlow as shown in Figure B.3 to schedule the CAN communications with the battery BMS. The received CAN messages from the battery to the handler includes (i) the battery voltage level, (ii) negative and positive contactors status, and (iii) battery cell temperatures. Then, the handler send required commands including (i) BMS wakeup, (ii) fan, and (iii) negative and positive connector actuation.

\section{B.3 HV Box}

As shown in Figure B.4, the HV line of the battery is connected to the HV Box input. Also the low voltage (i.e., $12 \mathrm{~V}$ ) cable of the Battery Management System goes to the low voltage Input of the HV Box. The inputs and outputs of the HV Box are discussed in detail below. The output from HV Box is connected to inverter/charger. Charger is connected when we need to charge the battery, or else, it is hooked off and Inverter is connected to the HV Box output. The main purposes of the HV Box are: 


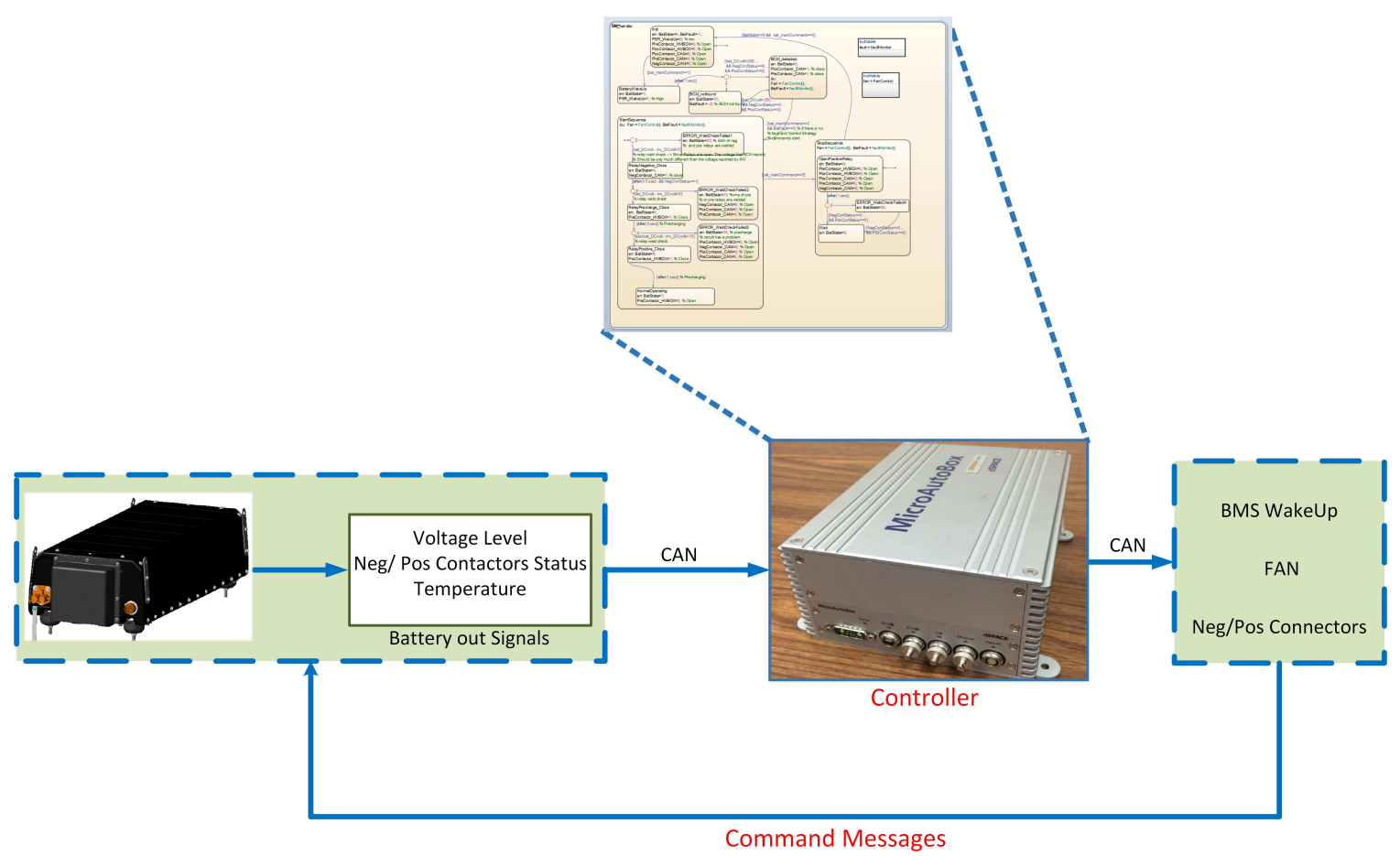

Figure B.3: Developed Battery Handler Software Layout

- Providing pre-charge Circuit for the Inverter

- Organizing the Batteries HV and LV harnesses

- Enabling usage of two battery pack in parallel

- LED indicator for pre-charge relays and battery fan modes

- Increasing the control signal current level for enabling the relays actuation

- To channel the output of battery to the charger for battery charging

The pre-charge circuit is necessary for the initial connection of the battery and the 
inverter due to the large initial voltage difference. In order to control and suppress the current flow, a $600 \Omega$ resistance is used in series with the battery and inverter. The pre-charge circuit will be deactivated after the voltage difference become less than $5 \mathrm{~V}$. Then, the pre-charge circuit relay will be opened and the battery will be connected directly to the inverter. Table B.1 lists the components used in building the HV Box.

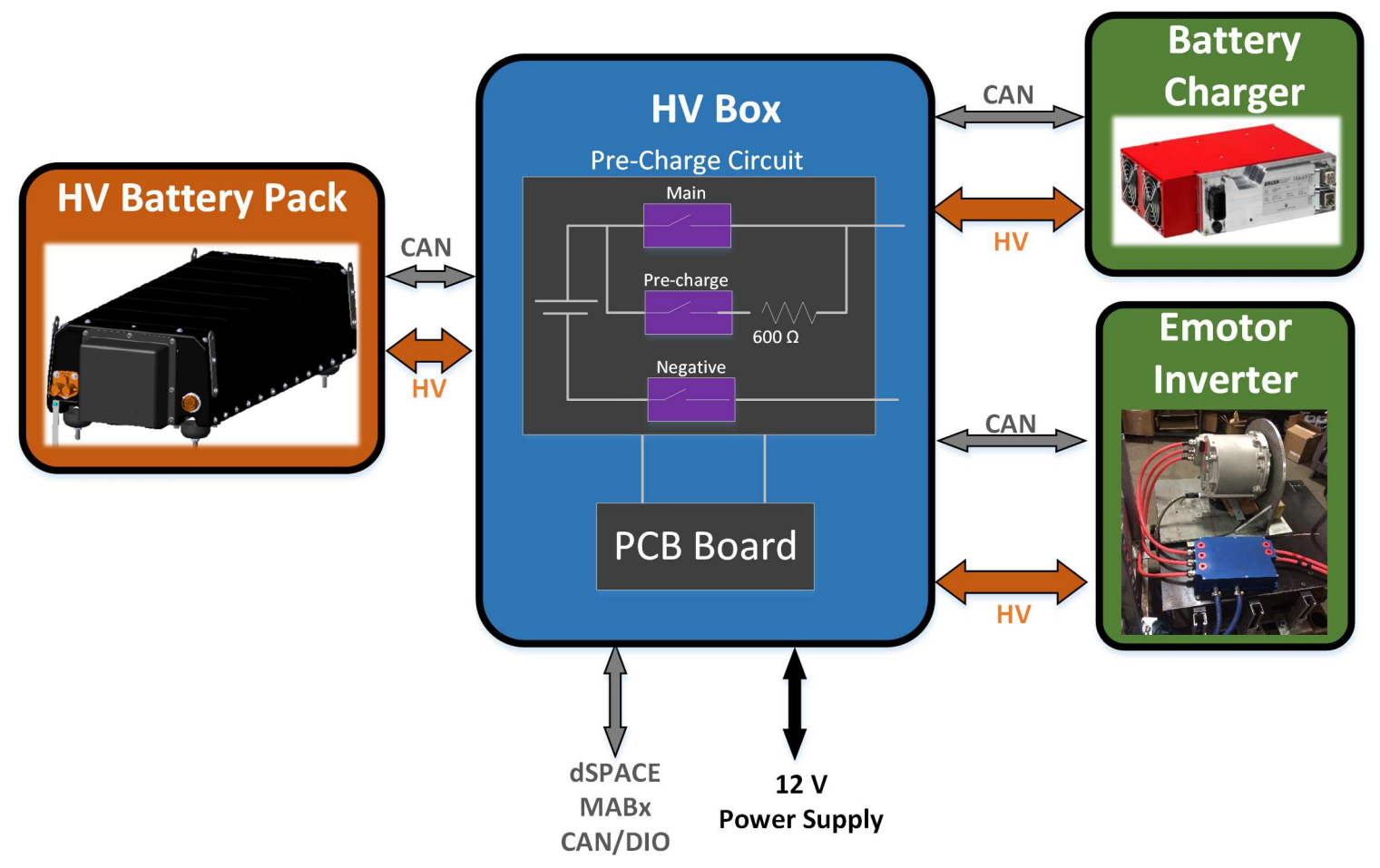

Figure B.4: HV box with its connected sub-systems. Details of the precharge circuit are also indicated in the figure. DIO in this figure refers to digital input/output

The PCB board in the HV Box is used for boosting the current level of the MABx digital command signal to control pre-charge relay, main relay, battery wake up signal and battery fan control. Figure B.5 shows the schematic of the electronic circuit used in the HV box. 
Table B.1

HV Box bill of materials and specifications.

\begin{tabular}{lc}
\hline \hline Items & Descriptions \\
\hline I/O HV Connectores & Anderson Connector-SB 350 AMP housing 4/0, Wire gauge 350 Amp \\
HV Fuse & FWP-125 A \\
Main/Pre-charge Relay & Tyco Electronics KILOVAC EV200 Series 900 VDC \\
Resistor & Vishay Wire Wound Resistors-600 ohms \\
LV Connector & TE Amphenol 24 Pin - TE Connectivity/AMP 206837-1 AND 206838-3 (female) \\
Contactors Pins & TE Connectivity 66590-1 \\
Fuse Holder & BH-1133 \\
\hline \hline
\end{tabular}

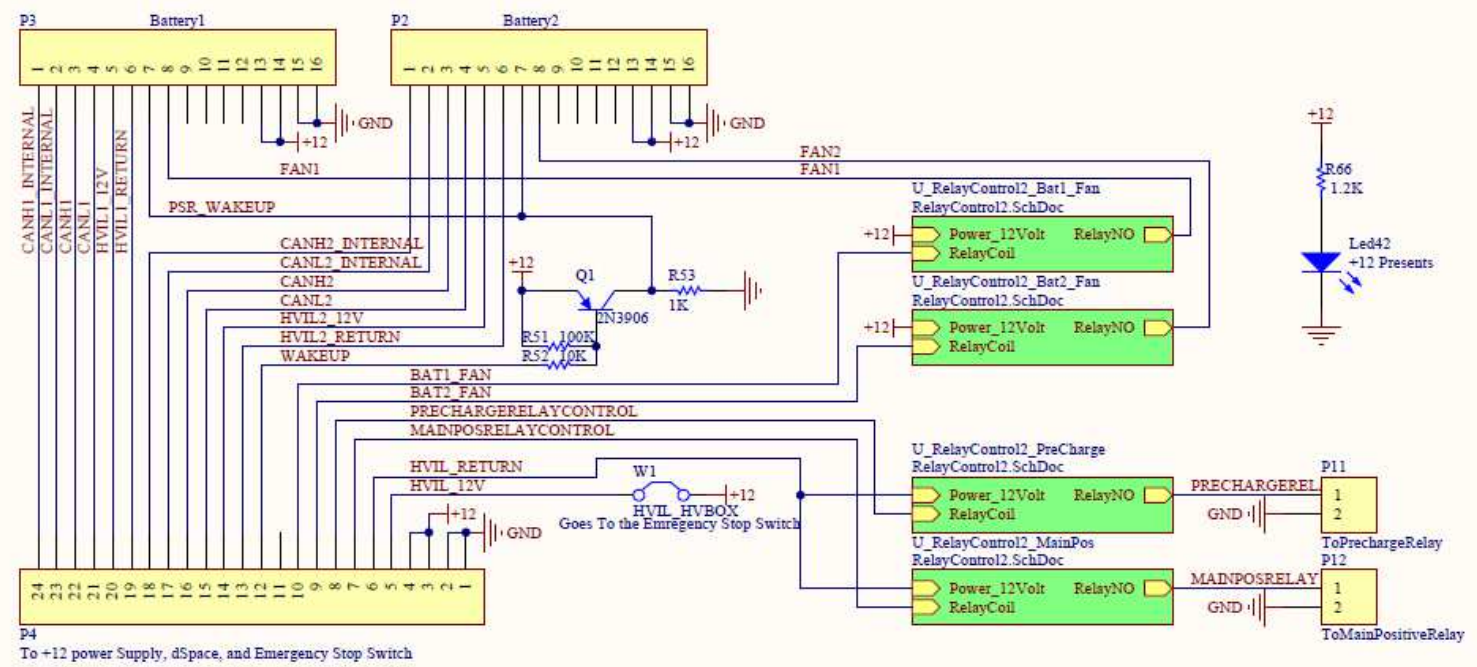

Figure B.5: Electric circuit of the designed HV box the pre-charge circuit.

The input to the board is from the 24 PIN Amphenol connector which is connected to P4 pins labeled in Figure B.5 in the same number fashion. These 24 wires are also described in the schematic. There are four $12 \mathrm{~V} \mathrm{LV}$ power relays in the PCB Board; relay 1 output goes to main positive HV relay; relay 2 connects to pre-charge HV relay; LV relay 3 output goes to Battery 2 fan signal and LV relay 4 output is connected to Battery fan signal. 


\section{B.4 Cooling System}

The inverter cooling is based on water and glycol and the e-motor is oil-based coolant. Thus, two different cooling loops were required for the inverter and e-motor. Table B.2 lists the cooling system components.

Table B.2

E-motor cooling system specifications.

\begin{tabular}{lc}
\hline \hline Items & Descriptions \\
\hline Oil Pump & APEX Oil Transfer Pump GR260101 \\
Oil Pump Maximum Pressure (psi) & 65 \\
Reservoir Capacity (Liter) & 1.9 \\
Two Heat Exchangers & Perma-Cool Maxi-Cool Six Pass Fluid Coolers 123182 \\
Check Valve & 8 AN inlet/outlet \\
\hline \hline
\end{tabular}

Figures B.6 and B.7 show the cooling loops for both the e-motor and inverter. The cooling loop operates continuously during the tests to keep the e-motor and inverter in required operating conditions. 


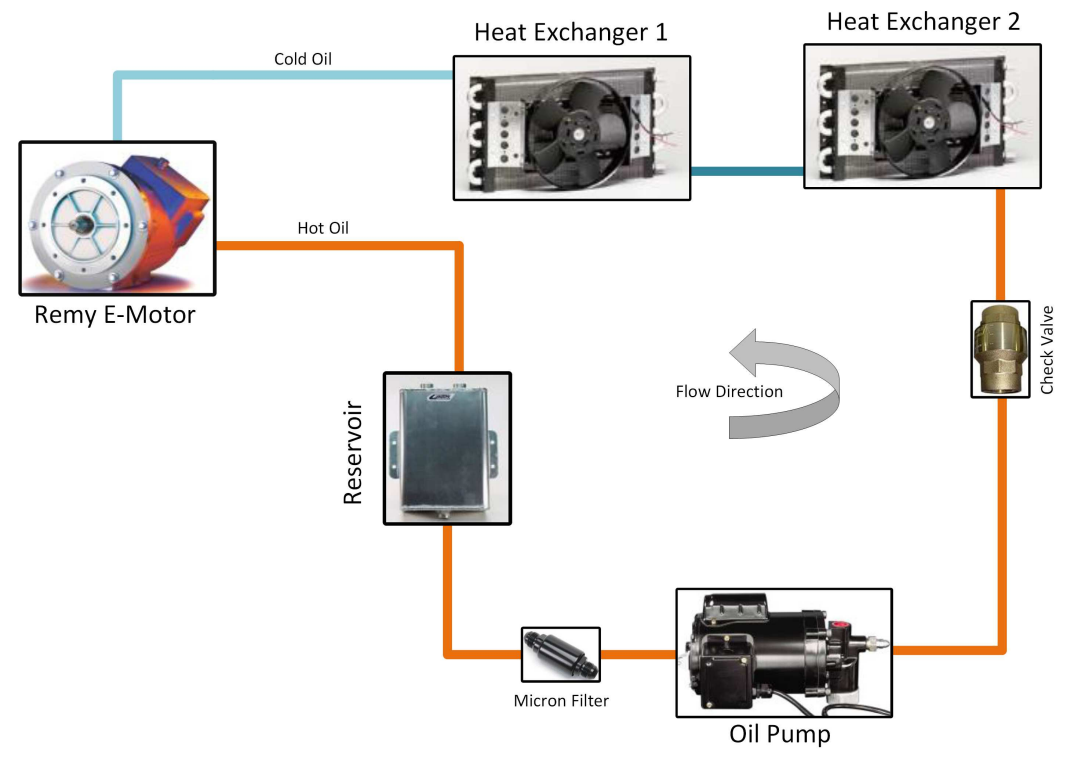

Figure B.6: E-motor cooling loop layout.

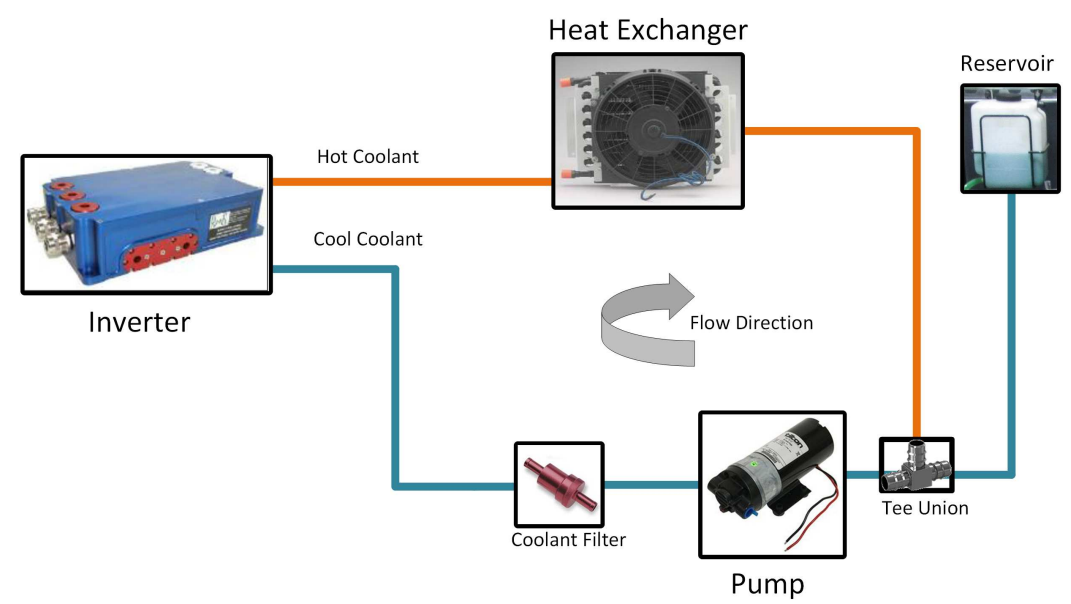

Figure B.7: Inverter cooling loop layout.

Figure B.8 illustrates the completed and built cooling systems. 


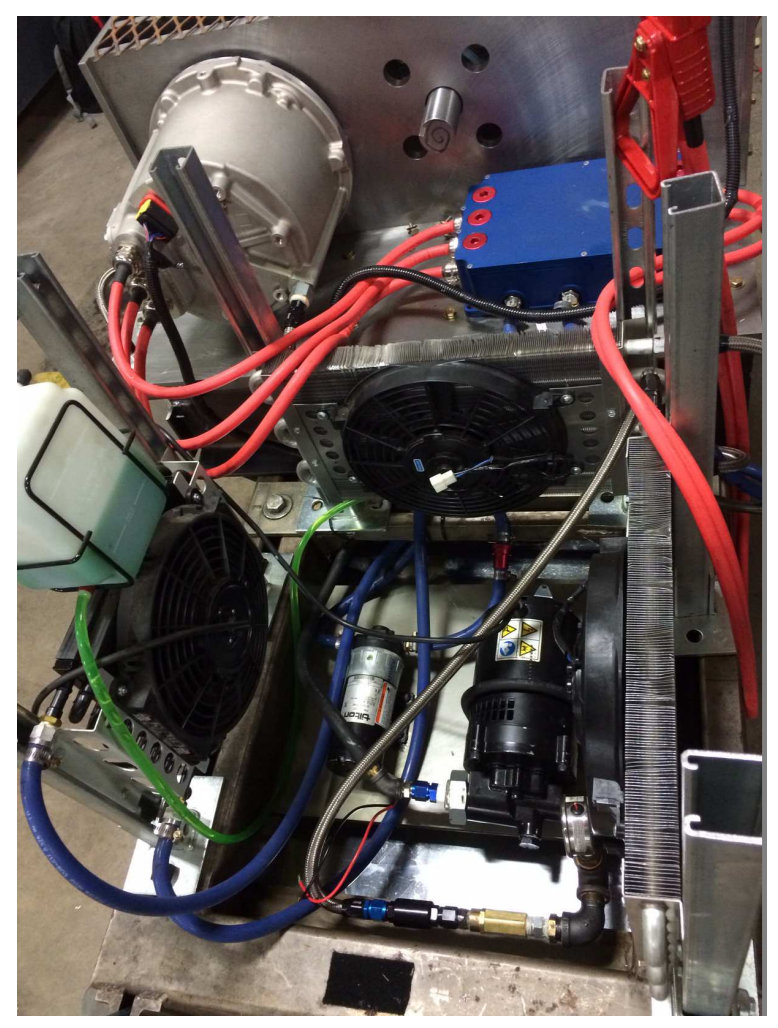

Figure B.8: Inverter cooling loop layout.

\section{B.5 Battery Charger}

Figure B.9 shows the battery charger used in this research. The aim of the battery charger is to charge the LG Chem battery for the tests. The battery charger is BRUSA NLG514 and its specifications are listed in Table B.3. 


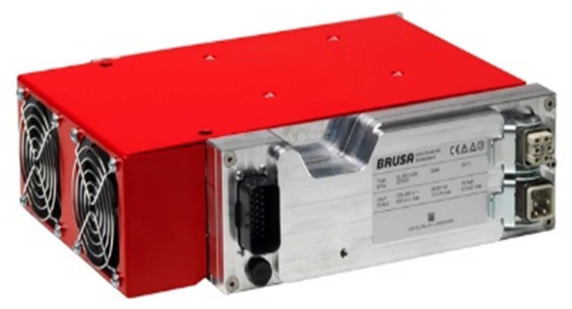

Figure B.9: BRUSA NLG514 $3.3 \mathrm{~kW}$ battery charger.

Table B.3

Battery charger specifications.

\begin{tabular}{lc}
\hline \hline Items & Descriptions \\
\hline Maximum Charging Power (kW) & 3.3 \\
Voltage Range (V) & $300-720$ \\
Maximum charging current (amp) & 9 \\
Maximum Efficiency (\%) & 93 \\
Mass (kg) & 6.3 \\
\hline \hline
\end{tabular}

Harness was made to connect the battery charger to both the battery and computer.

The harness has 10 pins and it is connected to the battery BMS. The connection to the computer is through RS232 serial connector (shown in Figure B.10). Battery charging begins by connecting the battery charger output to the HV box and connecting the HV box to the battery input. 


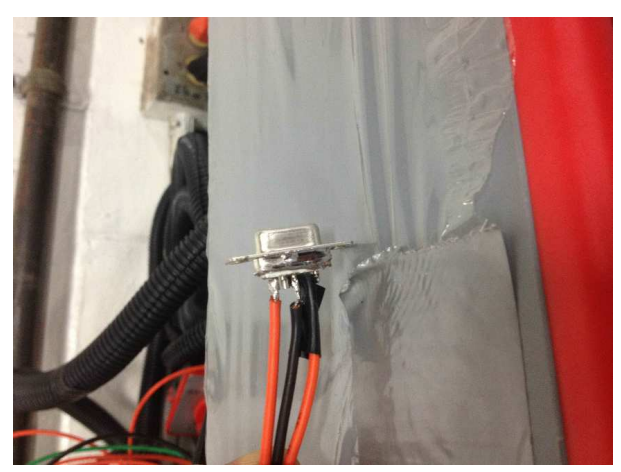

Figure B.10: RS232 wire used for communicating with the charger cubcontroller.

The Figure B.11 shows the charger profile used for the LGChem batteries charging.

The charging process first starts with constant current phase (i.e., $I_{1}$ ) by providing 9 A current, and once the battery voltage reached to $400 \mathrm{~V}$, it switches to constant voltage phase (i.e., $\left.U_{1}\right)$.

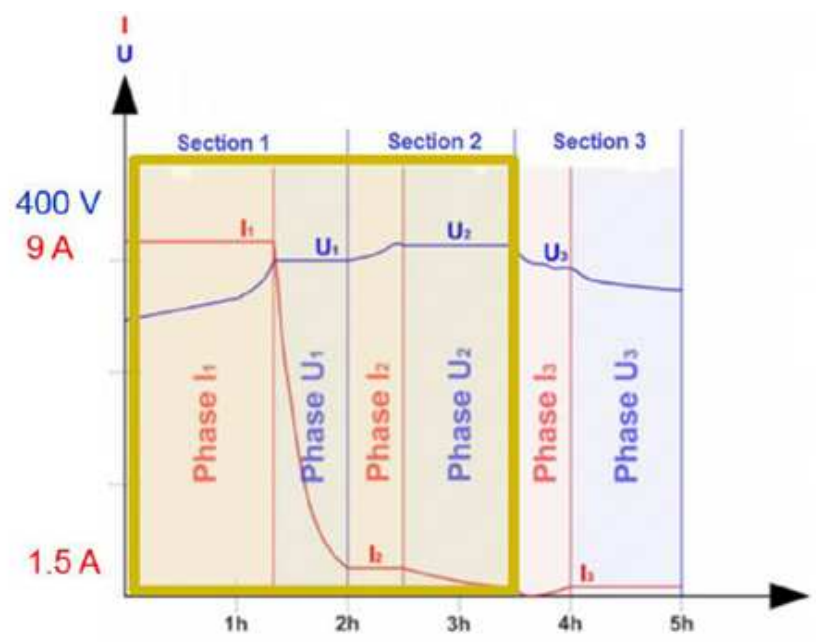

Figure B.11: Battery charging profile.

Below procedure describes the charging process: 
$\dagger$ Connect the RS232 connector to PC and make the connection through Hyper

Terminal software find on Windows (see Figure B.12) .

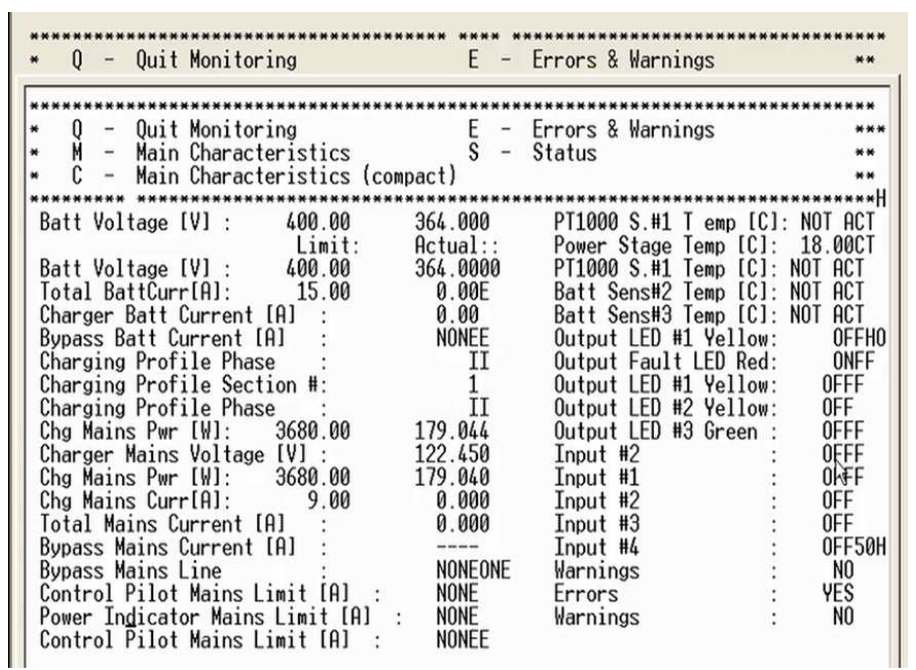

Figure B.12: Hyper Terminal software interface is used to send and read the charger controller signals.

$\dagger$ Power on the battery charger.

$\dagger$ Use ChargeStar software to load the charging profile on the battery charger controller. 



\section{Appendix $\mathrm{C}$}

\section{PhD Publications}

\section{C.1 Peer Reviewed Journal Papers}

\section{C.1.1 Published Journal Papers}

1. A. Solouk, M. Shahbakhti, "Energy Optimization and Fuel Economy Investigation of Series Hybrid Electric Vehicle Integrated with Diesel/RCCI Engines," Energies, 23 pages, Vol. 9, No. 12, 2016. (Ref. [2])

2. A. Solouk, M. Shahbakhti, "Modeling and Energy Management of an HCCI based Powertrain for Series Hybrid and Extended Range Electric Vehicles ," Int. J. of Powertrains, 29 pages, Vol. 6, No. 2, 2017, 
DOI:10.1504/IJPT.2017.10001761. (Ref. [1])

3. A. Solouk, M. Shakiba, M. Shahbakhti, "Analysis and Control of a Torque Blended Hybrid Electric Powertrain with a Multi-Mode LTC-SI Engine," SAE Int. J. of Alternative Powertrains, 15 pages, 6(1):2017, doi:10.4271/2017-011153, 2017. (Ref. [93])[Accepted in Feb. 2017]

\section{C.1.2 Under-Review Journal Paper}

1. A. Solouk, M. Shakiba, M. Shahbakhti, "Energy Analysis and Optimization of an Electrified Powertrain with a Multi-Mode High Efficiency Engine in Various Levels of Hybridizations," Applied Energy, 49 pages, Dec. 2016.

\section{C.1.3 Pre-Submission Journal Papers}

1. A. Solouk, J. Tripp, M. Shakiba, M. Shahbakhti, "Fuel Economy Assessment of an Extended Range Electric Vehicle with a Multi-Mode Low Temperature Combustion Engine," Energy Conversion and Management, to be submitted in Feb. 2017.

2. A. Rezaei, J. Burl, A. Solouk, M. Rezaei, M. Shahbakhti, "A Novel Real-Time Optimal Energy Management Strategy for Series Hybrid Electric Vehicles," Applied Energy, to be submitted in March 2017. 


\section{C.2 Peer Reviewed Conference Papers}

1. A. Solouk, M. Shakiba, M. Shahbakhti, "Analysis and Control of a Torque Blended Hybrid Electric Powertrain with a Multi-Mode LTC-SI Engine," Society of Automotive Engineers (SAE) Technical Paper No. 2017-01-1153, 15 pages, Detroit, MI, USA, April 4-6, 2017. (Ref. [94])[Accepted in Jan. 2017]

2. A. Solouk, M. Shakiba, K. Kannan, H. Solmaz, M. Bidarvatan, N. T. Kondipati, P. Dice, M. Shahbakhti, "Fuel Economy Benefits of Integrating a MultiMode Low Temperature Combustion (LTC) Engine in a Series Extended Range Electric Powertrain," Society of Automotive Engineers (SAE) Technical Paper 2016-01-2361, 15 pages, Baltimore, Maryland, USA, 2016. [Received the best presentation award of the conference - see Figure C.1] (Ref. [10])

3. A. Solouk, M. Shahbakhti, M. J. Mahjoob, "Energy Management and Control of a Hybrid Electric Vehicle with an Integrated Low Temperature Combustion (LTC) Engine," ASME Dynamic Systems and Control Conference (DSCC), 10 pages, October 22-24, 2014, San Antonio, Texas, USA. (Ref. [95])

\section{C.3 Abstract Referred Conference Papers}

1. A. Solouk, M. Shahbakhti, "Fuel Economy Benefits of Electrified Powertrains 
with Advanced Combustion Engines: Mild to Strong HEV," 2017 SIA Powertrain, Jun 7-8, Versailles, France. [Accepted in Dec. 2016]

2. A. Solouk, M. Shahbakhti, "Potential of Low Temperature Combustion (LTC) Engine Technology for Range Extender Vehicles," 2016 SAE Range Extenders for Electric Vehicles Symposium, Nov. 2, 2016, Knoxville, TN, USA. [This work received special attention and was selected/highlighted by GreenCarCongress.com, well-recognized media in the area of automotive engineering - see Figure C.2.

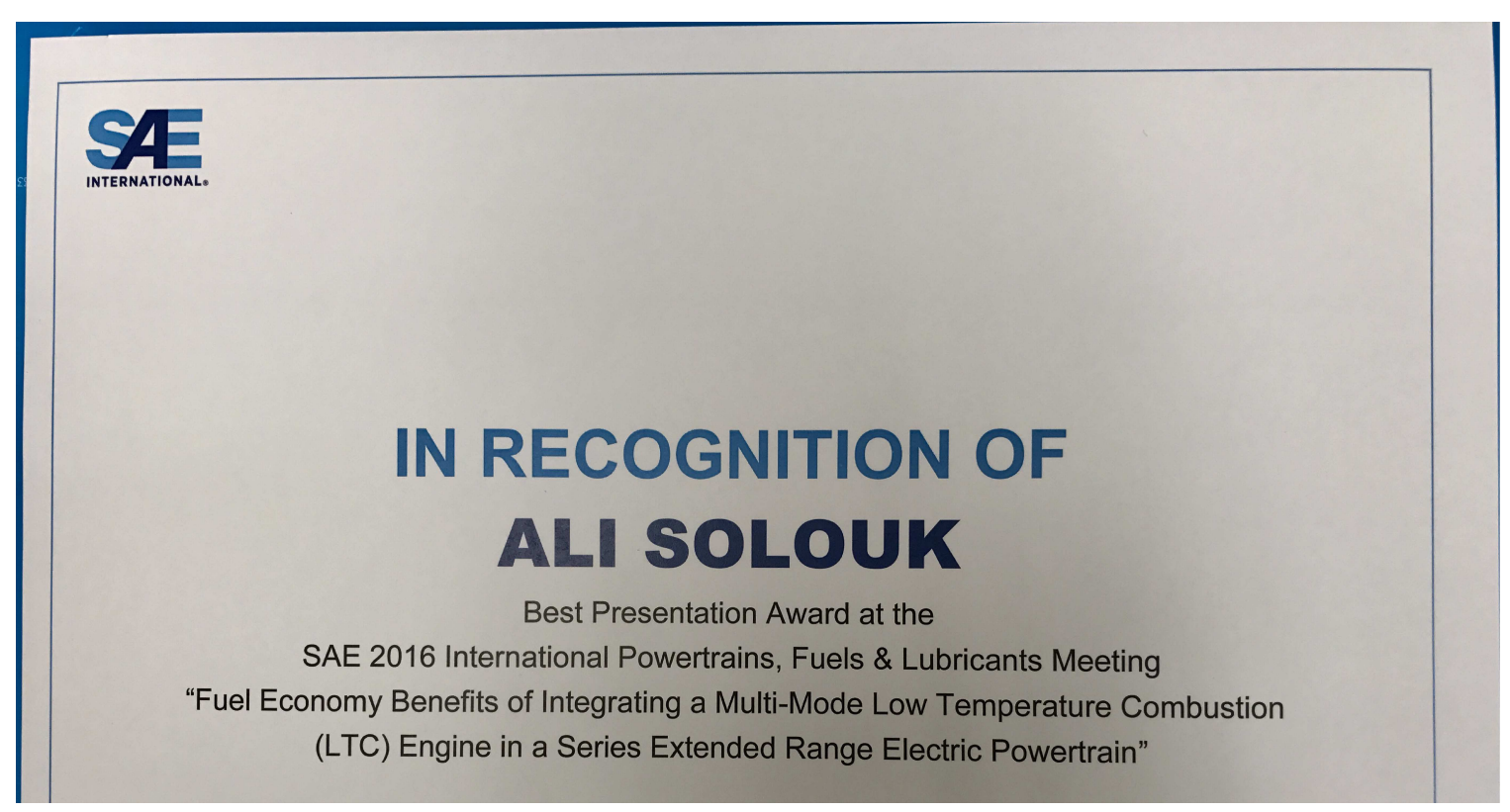

Figure C.1: SAE best presentation award received at SAE 2016 International Powertrains, Fuels, and Lubricants for reference [10]. 


\section{Green Car Congress}

Energy, technologies, issues and policies for sustainable mobility

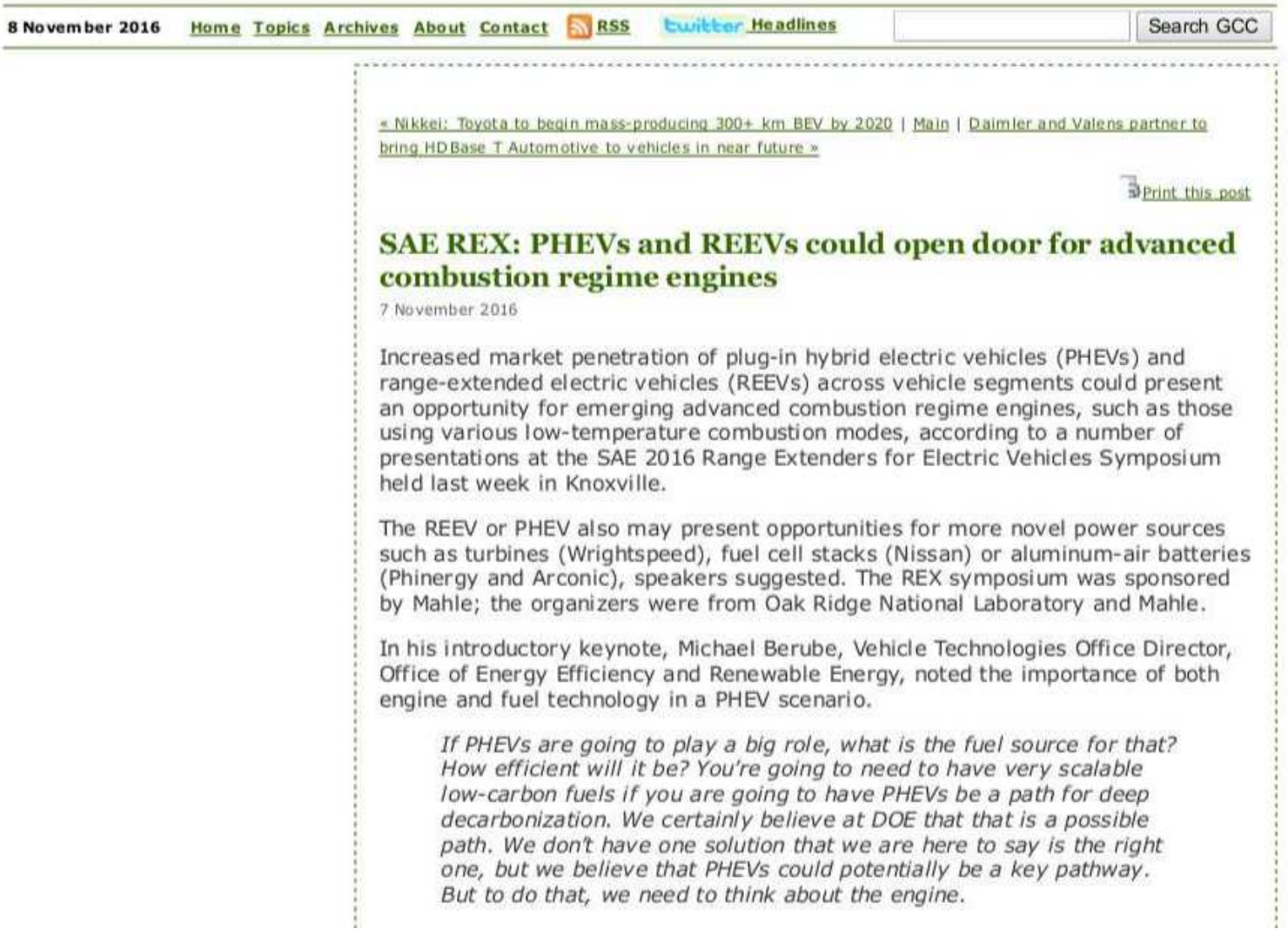


One example of the potential for low-temperature combustion (LTC) engine technology for range extended electric vehicles (REEV) presented at the Symposium was work by Ali Solouk and Assistant Professor Mahdi Shahbakhti at Michigan Technological University.

The category of LTC spans a range of specific technologies; these are of great interest as they promise to improve fuel economy, and reduce $\mathrm{NO}_{\mathrm{x}}$ and soot emissions by improving the in-cylinder combustion process.

LTC technology faces two main difficulties: first, a narrow operating range, which limits the use of the technology in conventional powertrains; second, complex combustion control, particularly durient transient operations. However, when applied in a range-extending application (i.e., as a generator), an LTC engine can work in a narrow operating area and increases the range of the battery pack.

Souk and Shahbakhti modified a turbocharged 2-liter GM Ecotec direct injection engine to function as a multi-mode LTC/Spark Ignition engine. The LTC modes included homogenous charge compression ignition ( $\mathrm{HCCI}$ ) and reactivity controlled compression ignition (RCCI) LTC engine.

Major changes to the base engine included:

- design and programming of a new Engine Control Unit (ECU)

- the addition of a port fuel injection system

- the capability to adjust intake temperature, pressure, and dilution level through the use of an intake air heater, supercharger, and Exhaust Gas Recirculation (EGR) rate modulation, respectively.

The engine functions as a generator for the battery pack.

Optimization results showed that in the UDDS driving cycle, the single-mode $\mathrm{HCCI}$ and RCCI engines offer $12 \%$ and $9 \%$ fuel economy improvement, respectively over a single-mode SI engine in the REEV. These improvements increase to $13.1 \%$ and $10.3 \%$ in the HWFET driving cycle. This fuel economy improvement is reduced

to $3 \%$ in comparison to a modern CI (i.e. diesel) engine in the HWFET driving cycle.

Simulation results showed that the LTC engine offers higher fuel economy improvement in more aggressive driving cycles (e.g., US06) compared to less aggressive driving cycles (e.g., UDDS).

The optimal control algorithm enhanced the fuel economy by $17.0 \%$ over the rulebased strategy in a hybrid electric vehicle integrated with an LTC engine.

Using a "multi-mode"-i.e., both HCCI and RCCI-instead of "single-mode" LTC engine in the REEV provided $2 \%$ more fuel economy improvement, depending on the type of the driving cycle. Reducing the mode-switching fuel penalty increased the fuel economy improvement beyond $2 \%$.

Among the LTC modes, HCCI was the dominant engine operating mode. If the fuel penalty to the RCCI mode decreased by $95 \%$, the engine operated near to $45 \%$ of its ON time in the RCCI mode.

The study also found that HCCI and RCCI were more favorable from the NVH considerations, compared to the SI engine. This is due to use of low engine speeds in the LTC modes.

The team will be presenting a new paper on their work at the SAE 2017 World Congress next year.

Resources

- Ali Solouk, Mohammad Shakiba-herfeh, Kaushik Kannan, Hamit Solmaz, Pau Dice, Mehran Bidarvatan, Naga Nithin Teja Kondipati, Mahdi Shahbakhti (2016) "Fuel Economy Benefits of Integrating a Multi-Mode Low Temperature Combustion (LTC) Engine in a Series Extended Range Electric Powertrain" SAE 2016-01-2361

Figure C.2: Paper [10] from this thesis is highlighted in GreenCarCongress.com, well-recognized media in the area of automotive engineering [10]. [URL: http://www.greencarcongress.com/2016/11/2016.html] 


\section{Appendix D}

\section{Letters of Permission}


* Versions of a paper defined as

- Author's Original $=$ Author's manuscript prior to peer review

- Accepted Manuscript $=$ Accepted version of Author's manuscript

- Proof $=$ Author's version of corrected accepted version

- Version of Record = Publisher's version of finished Article

In the case of 3 to 7 above, these rights are intended to help you in your academic and professional work, and are subject to two conditions:

a. These rights may not be used for commercial purposes; and

b. Acknowledgement in the form of a full citation must be given to the Journal as the original source of publication, together with a link to the Journal web page and/or DOI as soon as they are available.

You must obtain written permission beforehand from Inderscience for the following:

1. Copies of your Article in a course pack for onward commercial sale by a third party.

2. Except as specifically provided above, distribution in print or electronic form by any third party such as the convenor of a conference, database aggregator, website, blog or listserv;

3. Any other commercial use.

\section{What happens when the Author does not own copyright in the Article?}

If you have written the Article in the course of your employment, your employer may consider that it owns the copyright and is considered the legal Author. This is often referred to as 'Work for Hire'. In such cases, your employer should sign the Author Copyright Agreement. This does NOT apply to faculty and researchers working in a university and submitting the Article as a normal part of research activity.

If you are a government employee, the following applies:

1. If you are a UK, Canadian or Australian Government employee

Crown Copyright applies to your Article, and it should be cleared for publication by your Head of Department. This applies in the UK, Australia, Canada, New Zealand and eleven other Commonwealth countries. For full information, see the Office of Public Sector Information guidelines: http://www.opsi.gov.uk/advice/crown-copyright/copyrightguidance/publication-of-articles-written-by-ministers-and-civil-servants

2. If you are an officer or employee of the US Government

An Article prepared by an officer or employee of the US Federal Government does not enjoy copyright protection under US law; it is 'non-copyright', so there is no copyright to transfer. It is your responsibility to make the necessary enquiries and provide Inderscience with any form of words that is required for publication. Please note that this applies only to Federal, and not to state or local government employees.

If your research has been funded by a grant from a research funding agency, the funding grant may have had conditions attached to it that affect the transfer of copyright to Inderscience, or require you to post you Article to a repository. It is your responsibility to advise Inderscience of any such conditions and provide any documentation that may be required before publication.

What are the Author's responsibilities?

2015 Inderscience Enterprises Ltd.

Figure D.2: Copyright permission letter for using the materials from [1] manuscript used in Chapter 2 


\section{D.2 Letter of Permission for [2] (Chapter 3)}

Michigan Tech
Ali Solouk<asoloukm@mtu.edu>

Permission for Energies-154999

Ali Solouk<asoloukm@mtu.edu>

Thu, Dec 1, 2016 at 9:54 PM

Hello,

I am contacting you to request for copyright permission for my accepted manuscript in Energies with number 154999 . The publication title is:

"Energy Optimization and Fuel Economy Investigation of a Series Hybrid Electric Vehicle Integrated with Diesel/RCCI Engines". The article is currently under proofreading and will be an open source article.

I want to use the manuscript as one of my PhD dissertation's chapter and I want to get the copyright permission for that.

Could you let me know what the policy is for granting the permission to use the article materials in my dissertation?

I look forward to hearing back from you

Best Regards,

Ali Solouk

Ali Solouk, PhD Candidate

Research Assistant, Energy Mechatronics Lab (EML) and APS

(a) Department

www.linkedin.com/in/alisolouk/

Support MDPI <support@mdpi.com>

Fri, Dec 2, 2016 at 6:53 AM

To: Ali Solouk <asoloukm@mtu.edu>

Dear Dr. Solouk,

Thank you very much for your interest in said material.

All MDPI journals are Open Access and subject to the Creative Commons Attribution License (CC BY). The CC BY permits unrestricted use, distribution, and reproduction of the material in any medium, even commercially, ${ }^{*}$ provided the original work is properly cited ${ }^{\star}$. You do not have to pay anything for permission.

For more information on the CC BY License, please see here: https://creativecommons.org/licenses/by/4.0/legalcode

Best regards

Larissa

[Quoted text hidden]

Figure D.3: Copyright permission letter for using the materials from [2] manuscript used in Chapter 3 


\section{D.3 Letter of Permission for Data Used in Figures}

\subsection{1 and 4.12 (Chapter 4)}

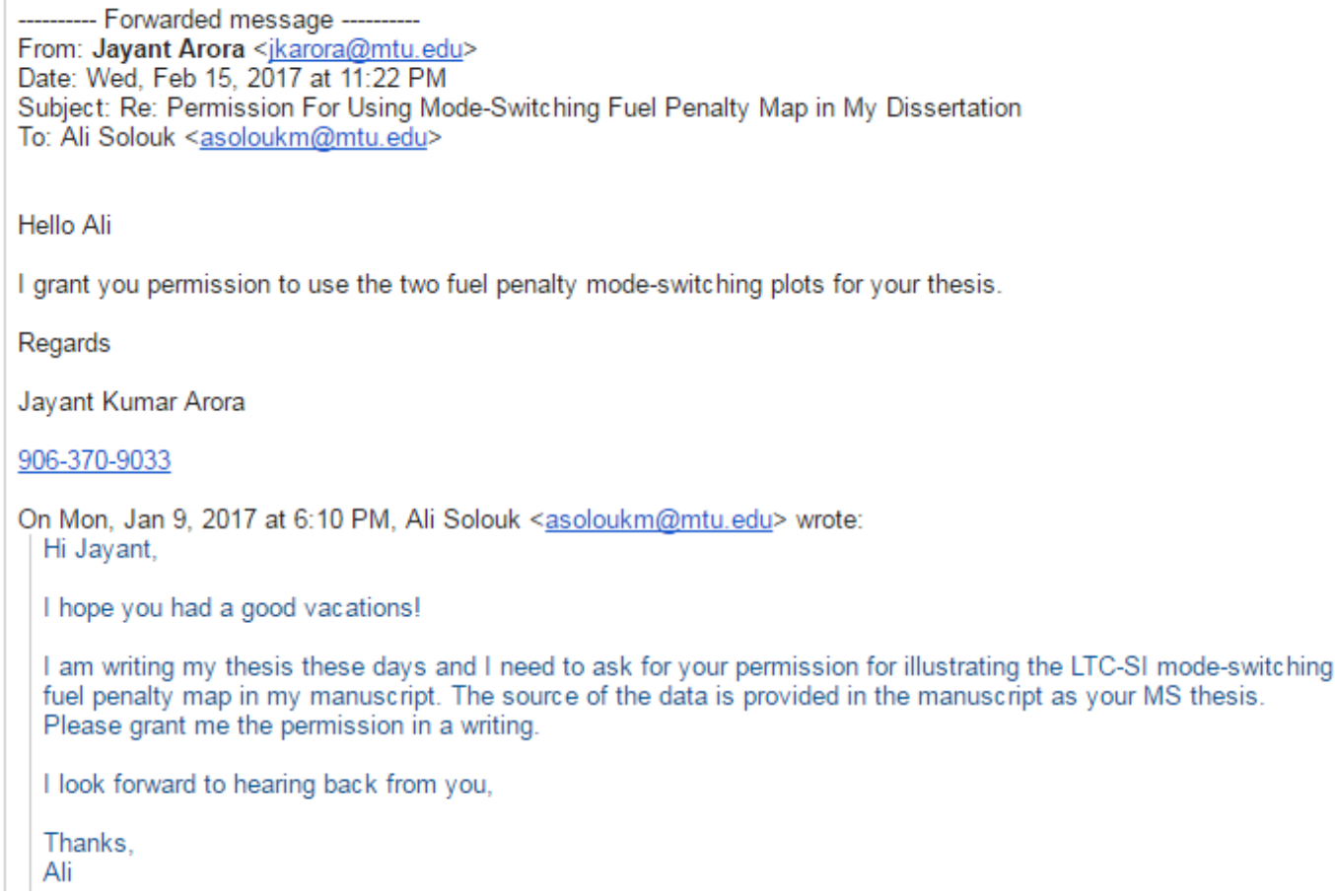

Figure D.4: Permission letter from Jayant Arora for using the data in Figures 4.11 and 4.12 as part of the study in [9]. (Chapter 4) 


\section{D.4 Letter of Permission for [3, 4] (Chapters [5] and}

6 )

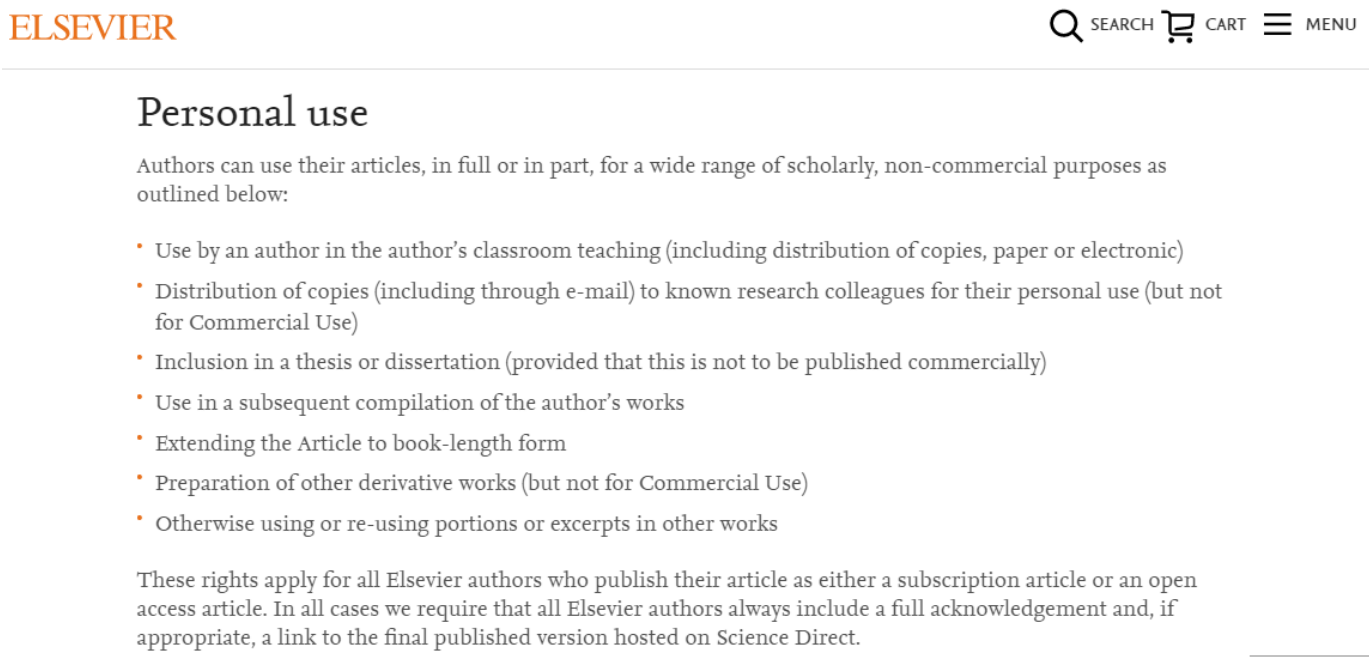

Figure D.5: Copyright permission for using the materials from [3, 4] manuscript used in Chapters [5] and [6] 


\section{Appendix E}

\section{Program and Data File Summary}

Following files were used for this dissertation arranged in the tables.

\section{E.1 Chapter 1}

Table E.1

Chapter 1 figure files.

\begin{tabular}{cc}
\hline \hline File name & File description \\
\hline Introduction_EngineHEV_5_rev.eps & Figure 1.1 \\
ChapterOrganization.eps & Figure 1.2 \\
\hline \hline
\end{tabular}




\section{E.2 Chapter 2}

Table E.2

Chapter 2 figure files.

\begin{tabular}{cc}
\hline \hline File name & File description \\
\hline Introduction_EngineHEV_6.eps & Figure 2.1 \\
HCCI_Eng_scheme_v11.eps & Figure 2.2 \\
HCCI_Map_Combined.eps & Figure 2.3 \\
Acc_Braking_Validation_Revised_1.eps & Figure 2.4 \\
EM_Speed_Torque_Simplified_Model_Validation_n.eps & Figure 2.5 \\
Battery_Validation.eps & Figure 2.6 \\
SI_Map.eps & Figure 2.8 \\
Motor_Speed.eps & Figure 2.9 \\
EM_Op_Eff.eps & Figure 2.10 \\
Driver.eps & Figure 2.11 \\
SOC_On_Off_TRB_DP.eps & Figure 2.12 \\
Power_Dist.eps & Figure 2.13 \\
SOC_2.eps & Figure 2.14 \\
FE_SinglePoint.eps & Figure 2.15 \\
OperatingPoint_Impact_T.eps & Figure 2.16 \\
On_OFF_PHEV_SHEV_DP.eps & Figure 2.17 \\
DrivingCycleImpact.eps & Figure 2.18 \\
\hline \hline
\end{tabular}


Table E.3

Required data files.

\begin{tabular}{cc}
\hline \hline File name & File description \\
\hline $\begin{array}{c}\text { Impact of Operating Points } \\
\text { and Fuel Penalty.xlsx }\end{array}$ & Fuel economy variation in \\
& various engine startup fuel penalty \\
Impact of Driving cycle & Fuel economy variation in \\
and SHEV and EREV.xlsx & various driving cycle \\
RCCI_MPC.xlsx & MPC results \\
\hline \hline
\end{tabular}

Table E.4

MATLAB script and SIMULINK files.

\begin{tabular}{cc}
\hline \hline File name & File description \\
\hline Ali_UQM_DP.mdl & Series HEV Simulink model \\
Batt_Tech_Specs.m & Battery data \\
Generator_Tech_Specs & Generator data \\
ICE_Tech_Specs.m & Engine data \\
Student_Design_Parameters.m & Design Script \\
UDDS_HWFET_UDDS_US06_UDDS.mat & Combined Driving Cycle \\
Model_MPC.m & MPC Run Script \\
Model_DP.m & DP Run Script \\
EM.m & E-motor efficiency map with operating points \\
Analysis_Plots_T.m & Fuel Economy Sensitivity with Fuel Penalty \\
PowerAnalysis.m & Power distribution plot script \\
\hline \hline
\end{tabular}




\section{E.3 Chapter 3}

Table E.5

Chapter 3 figure files.

\begin{tabular}{cc}
\hline \hline File name & File description \\
\hline Introduction_EngineHEV_9.eps & Figure 3.1 \\
RCCI_Eng_scheme_v2.eps & Figure 3.2 \\
RCCI_Map.eps & Figure 3.3 \\
CI_Map_Revised.eps & Figure 3.4 \\
SI_Map_Revised.eps & Figure 3.5 \\
RCCI_TempMap.eps & Figure 3.6 \\
Driving_Cycle.eps & Figure 3.7 \\
Operating_Mode_Selection_CI_SI_RCCI_T.eps & Figure 3.8 \\
Motor_Speed.eps & Figure 3.9 \\
EM_Op_Eff.eps & Figure 3.10 \\
Driver.eps & Figure 3.11 \\
SOC_On_Off_TRB_DP_rev.eps & Figure 3.12 \\
Power_Dist.eps & Figure 3.13 \\
SOC.eps & Figure 3.14 \\
RCCI_MPC_SI_CI_Comb.eps & Figure 3.15 \\
Batt_Ploss_EREV_SHEV.eps & Figure 3.16 \\
EREV_SHEV_TimeDifference.eps & Figure 3.17 \\
MPC_EREV_SHEV_rev.eps & Figure 3.18 \\
Driving_cycle_and_SHEV_and_EREV_T.eps & Figure 3.19 \\
\hline \hline
\end{tabular}


Table E.6

MATLAB script and SIMULINK files.

\begin{tabular}{|c|c|}
\hline File name & File description \\
\hline Ali_UQM_DP.mdl & Series HEV Simulink model \\
\hline Batt_Tech_Specs.m & Battery data \\
\hline Generator_Tech_Specs & Generator data \\
\hline ICE_Tech_Specs.m & Engine data \\
\hline Student_Design_Parameters.m & Design Script \\
\hline UDDS_HWFET_UDDS_US06_UDDS.mat & Combined Driving Cycle \\
\hline Model_MPC.m & MPC Run Script \\
\hline Model_DP.m & DP Run Script \\
\hline EM.m & $\begin{array}{l}\text { E-motor efficiency map } \\
\text { with operating points }\end{array}$ \\
\hline Analysis_Plots_T.m & $\begin{array}{l}\text { Fuel Economy Sensitivity } \\
\text { with Fuel Penalty }\end{array}$ \\
\hline Analysis_FuelEconomy.m & Plot script for fuel economy \\
\hline $\begin{array}{l}\text { Analysis_FuelEconomy } \\
\text { Improvement.m }\end{array}$ & $\begin{array}{l}\text { Plot script for } \\
\text { fuel economy improvement }\end{array}$ \\
\hline EngineSizing.m & Script engine sizing \\
\hline OP_ONOFF.m & $\begin{array}{c}\text { Script to plot engine } \\
\text { ON and OFF profile for different modes }\end{array}$ \\
\hline OperatingPoint_Impact.m & $\begin{array}{l}\text { Script to plot fuel economy } \\
\text { improvement over SI } \\
\text { and CI in different modes }\end{array}$ \\
\hline Power_Comb.m & $\begin{array}{l}\text { Script to plot SHEV } \\
\text { and EREV comparisons }\end{array}$ \\
\hline
\end{tabular}




\section{E.4 Chapter 4}

Table E.7

Chapter 4 figure files.

\begin{tabular}{cc}
\hline \hline File name & File description \\
\hline TestSetup_V3.eps & Figure 4.1 \\
Cart_CAD.eps & Figure 4.2 \\
Mount_Ansys.eps & Figure 4.3 \\
TestSetupLayout.eps & Figure 4.4 \\
Traction_Test_2.eps & Figure 4.5 \\
LGBatt_Specifications_Combined_N.eps & Figure 4.6 \\
Estimated_CombinedMap_Setup_Nov13.eps & Figure 4.7 \\
LTC_Setup_ForJournal_V2.eps & Figure 4.8 \\
LTC_SI_Engine_Torquebase_Map.eps & Figure 4.9 \\
CombinedEngine_Temp.eps & Figure 4.10 \\
run_86_600-900.eps & Figure 4.11 \\
HCCI_SI.eps & Figure 4.12 \\
ModeSwitching_FuelPenaltyMap.eps & Figure 4.13 \\
\hline \hline
\end{tabular}

Table E.8

Experimental data files for tests.

\begin{tabular}{cc}
\hline \hline File name & File description \\
\hline Amir_Ali_Combined_Model_Modified & Simulink model used for \\
_HVBoXTest_03.slx & Torque Tracking test \\
Amir_Ali_Combined_Model & Simulink model used for battery charging \\
_Modified & \\
_HVBoXTest.slx & and testing the HVBox. \\
\hline \hline
\end{tabular}




\section{E.5 Chapter 5}

Table E.9

Chapter 5 figure files.

\begin{tabular}{cc}
\hline \hline File name & File description \\
\hline Introduction_EngineHEV_V1.eps & Figure [5.1 \\
ExtendeRange_Schematic_V2.eps & Figure 5.2 \\
Engine_Costs.eps & Figure [5.3 \\
HCCI_OP.eps + RCCI_OP.eps + SI_OP.eps & Figure [5.4 \\
Combined_OptEngP_HWFET.eps & Figure [5.5 \\
EnergyAnalysis_SingleOP.eps & Figure 5.6 \\
FE_and_FEImprovement.eps & Figure 5.7 \\
NVH_UDDS.eps & Figure 5.8 \\
Multimode_ToSI.eps & Figure 5.9 \\
Multimode_ToRCCI.eps & Figure 5.10 \\
\hline \hline
\end{tabular}

Table E.10

Required data files.

\begin{tabular}{cc}
\hline \hline File name & File description \\
\hline RCCI_Naturally_Aspirated_BSFC.txt & RCCI engine BSFC Experimental data \\
SI_Naturally_Aspirated_BSFC.txt & SI engine BSFC Experimental data \\
PPCI_Naturally_Aspirated_BSFC.txt & PPCI engine BSFC Experimental data \\
\hline \hline
\end{tabular}


Table E.11

MATLAB script and Simulink files.

\begin{tabular}{cc}
\hline \hline File name & File description \\
\hline Series_PMP_Multimode.m & Multi-Mode Enigne PMP Optimization script \\
Series_PMP_FuelPenaltyAdded.m & Single-Mode PMP optimization run script \\
Battery_SOCdot_Estimator.m & Battery dynamic function \\
BSFC_min_selector.m & Script for selcting optimum engine speed \\
Engine_Map_Sorter.m & Script for generating engine bsfc maps \\
HCCI_CostFunction.m & Function to find different engines fuel consumption \\
LG_Powerlimit_Estimator.m & LG battery power limit estimator function \\
PMP_Plots.m & Script plot \\
fuelpenalty.m & Mode-switching fuel penalty data \\
Analysis.m & Script for results analysis and generating plots \\
Fuel_SOC_Compensation.m & Script to compensate for delta SOC in fuel \\
\hline \hline
\end{tabular}

\section{E.6 Chapter 6}

Table E.12

Chapter 6 figure files.

\begin{tabular}{cc}
\hline \hline File name & File description \\
\hline Introduction_EngineHEV_5.eps & Figure 6.1 \\
ParallelHEV_Schematic_V4.eps & Figure 6.2 \\
SingleMode_SI_OperatingPoints.eps & Figure 6.3 \\
MultiMode_OperatingPoints.eps & Figure 6.4 \\
ModeDistribution.eps & Figure 6.5 \\
FuelEconomy_T.eps & Figure 6.6 \\
Pbatt_Pdem_EngMode_Rev2.eps & Figure 6.7 \\
SingleMode_SI_OperatingPoints_EngTemperature.eps & Figure 6.8 \\
MultiMode_OperatingPoints_EngTemperature.eps & Figure 6.9 \\
\hline \hline
\end{tabular}


Table E.13

Required data files.

\begin{tabular}{cc}
\hline \hline File name & File description \\
\hline REMYMotor_Eff_Map.mat & Remy Motor Experimental Efficiency Data \\
Costate_FixBatteryCapacity_Journal.txt & Costate values foe each senario \\
RCCItoSI_FP.mat & RCCI to SI mode-switch fuel penalty \\
SItoHCCI_FP.mat & SI to HCCI mode-switch fuel penalty \\
HCCI_Modified.mat & HCCI engine experimental BSFC data \\
RCCI_Modified.mat & RCCI engine experimental BSFC data \\
SI_Modified.mat & SI engine experimental BSFC data \\
\hline \hline
\end{tabular}

Table E.14

MATLAB script and Simulink files.

\begin{tabular}{cc}
\hline \hline File name & File description \\
\hline $\begin{array}{c}\text { Parallel_PMP_Multimode_ER } \\
\text { _Variable_TemperatureConstraint.m }\end{array}$ & Multi-Mode Enigne PMP \\
Parallel_PMP_Single_SI & Optimization script with Temperature Constraint \\
_Mode_ER_Variable.m & Single-Mode Enigne PMP \\
Series_PMP_FuelPenaltyAdded.m & Optimization script for different hybridization levels \\
REMY_Eff_Estimator.m & Single-Mode PMP optimization run script \\
Battery_SOCdot_Estimator.m & Remy e-motor efficiency estimator function \\
HCCI_CostFunction.m & Battery dynamic function \\
LG_Powerlimit_Estimator.m & Function to find different engines fuel consumption \\
PMP_Plots.m & LG battery power limit estimator function \\
Analysis.m & Script plot \\
Script.m & Script for results analysis and generating plots \\
\hline \hline
\end{tabular}




\section{E.7 Appendix A}

Table E.15

Appendix A figure files.

\begin{tabular}{cc}
\hline \hline File name & File description \\
\hline EM_Size.eps & Figure A.1 \\
EM_Size_grade.eps & Figure A.2 \\
EM_SELECT.eps & Figure A.3 \\
Eng_ConstantV.eps & Figure A.4 \\
\hline
\end{tabular}

\section{E.8 Appendix B}

Table E.16

Appendix $\mathrm{A}$ figure files.

\begin{tabular}{cc}
\hline \hline File name & File description \\
\hline SoftwareCommunication_Layout.eps & Figure B.1 \\
BatteryHandler.eps + InverterHandler.eps & Figure B.2 \\
BatterCommunication_Layout.eps & Figure B.3 \\
HVBox.eps & Figure B.4 \\
PCBBoard.eps & Figure B.5 \\
Emotor_CoolingSys.eps & Figure B.6 \\
Inverter_CoolingSys.eps & Figure B.7 \\
CoolingSys_Built.eps & Figure B.8 \\
Charger.eps & Figure B.9 \\
RS232_Connector.eps & Figure B.10 \\
ChargingProfile.eps & Figure B.11 \\
HyperTerminal.eps & Figure B.12 \\
\hline \hline
\end{tabular}

